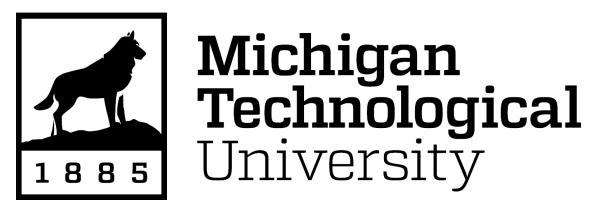

Michigan Technological University Digital Commons @ Michigan Tech

Dissertations, Master's Theses and Master's Reports

2020

\title{
Aperture-Level Simultaneous Transmit and Receive (STAR) with Digital Phased Arrays
}

lan Cummings

Michigan Technological University, itcummin@mtu.edu

Copyright 2020 lan Cummings

\section{Recommended Citation}

Cummings, lan, "Aperture-Level Simultaneous Transmit and Receive (STAR) with Digital Phased Arrays", Open Access Dissertation, Michigan Technological University, 2020.

https://doi.org/10.37099/mtu.dc.etdr/1014

Follow this and additional works at: https://digitalcommons.mtu.edu/etdr

Part of the Signal Processing Commons 
APERTURE-LEVEL SIMULTANEOUS TRANSMIT AND RECEIVE (STAR)

\title{
WITH DIGITAL PHASED ARRAYS
}

\author{
By \\ Ian T. Cummings

\begin{abstract}
A DISSERTATION
Submitted in partial fulfillment of the requirements for the degree of DOCTOR OF PHILOSOPHY

In Electrical Engineering
\end{abstract}

MICHIGAN TECHNOLOGICAL UNIVERSITY

2020

(C) 2020 Ian T. Cummings 

This dissertation has been approved in partial fulfillment of the requirements for the Degree of DOCTOR OF PHILOSOPHY in Electrical Engineering.

Department of Electrical and Computer Engineering

\author{
Dissertation Co-advisor: Dr. Timothy C. Havens \\ Dissertation Co-advisor: Dr. Timothy J. Schulz \\ Committee Member: Dr. Daniel R. Fuhrmann
}

Committee Member: Dr. Jonathan P. Doane

Department Chair: $\quad$ Dr. Glen Archer 



\section{Dedication}

To my Dad, my Mom, and the many teachers and mentors

who taught, guided, inspired, prayed, and loved me through the many stages of learning and life that I have encountered so far.

"Work willingly at whatever you do, as though you were working for the Lord rather than for people. Remember that the Lord will give you an inheritance as your reward, and that the Master you are serving is Christ." - Colossians 3:23-24 NLT 



\section{Contents}

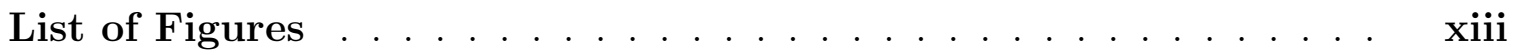

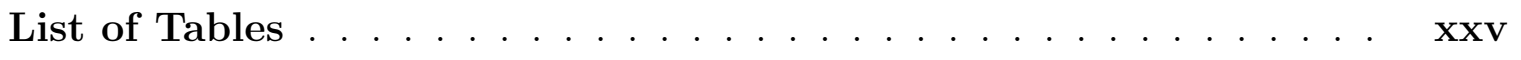

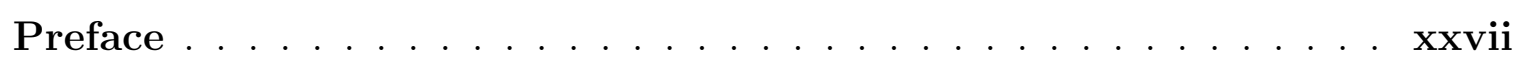

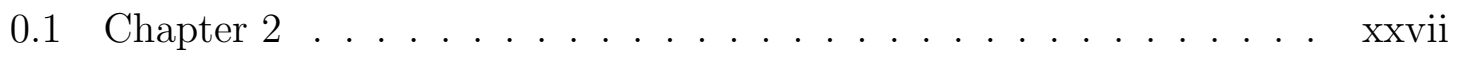

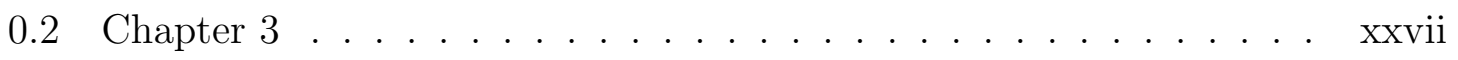

0.3 Chapter $4 \ldots \ldots \ldots \ldots \ldots$

Acknowledgments ................... $\ldots \ldots$

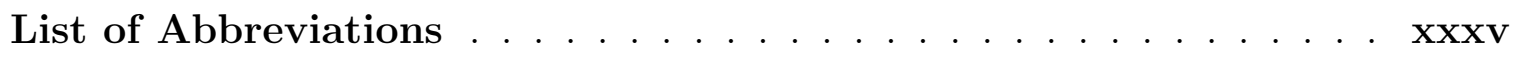

Abstract . . . . . . . . . . . . . . . . . . .

1 Introduction ..................... 1

1.1 Problem Context . . . . . . . . . . . . . . . . . . 2

1.2 Dissertation Outline and Contributions .......... 5

1.2 .1 Chapter $2 \ldots \ldots \ldots \ldots \ldots \ldots \ldots$

1.2 .2 Chapter $3 \ldots \ldots \ldots \ldots \ldots \ldots \ldots$. . . . . . . . . . . . 
1.2 .3 Chapter $4 \ldots \ldots \ldots \ldots \ldots \ldots \ldots \ldots \ldots \ldots \ldots \ldots \ldots \ldots$

1.2 .4 Chapter $5 \ldots \ldots \ldots \ldots \ldots \ldots$

1.3 List of Relevant Publications . . . . . . . . . . . . . . 8 8

2 Aperture-Level Simultaneous Transmit and Receive with Digital

Phased Arrays ...................... 11

2.1 Introduction . . . . . . . . . . . . . . . . . 11

2.1.1 Digital Adaptive Beamforming for STAR . . . . . . . 15

2.1 .2 Digital SIC for STAR . . . . . . . . . . . . 16

2.1.3 Advanced Digital Techniques for STAR . . . . . . . . 17

2.1.4 Previous Work, Contributions, \& Organization . . . . . . . 21

2.2 Aperture-Level Simultaneous Transmit and Receive (ALSTAR) Array 24

$2.2 .1 \quad$ System SINR $\ldots \ldots \ldots \ldots \ldots$

2.2.2 Mitigation of Transmitted Signal and Noise . . . . . . 29

2.2.3 Mitigation of Observation and Receive Noise . . . . . . . 33

2.3 Effective Isotropic Isolation $\ldots \ldots \ldots \ldots$

2.3.1 Properties of the Mutual Coupling Channel ... . . . . 41

2.4 Simulation Results $\ldots \ldots \ldots \ldots$

2.4 .1 Partition A . . . . . . . . . . . . 45

2.4 .2 Partition B . . . . . . . . . . . . . . 47

2.5 Beamformer Regularization $\ldots \ldots \ldots \ldots \ldots$

2.6 Conclusion . . . . . . . . . . . . . . . . . . . . . . . . . . 49 
3 Narrowband Direction-of-Arrival Estimation with Aperture-Level

Simultaneous Transmit and Receive Digital Phased Arrays . . . 63

3.1 Introduction . . . . . . . . . . . . . . . 63

3.1.1 DOA Related Work ............... 65

3.1.2 STAR Related Work . . . . . . . . . . . . . . 68

3.2 ALSTAR Array Signal Model . . . . . . . . . . . . . . . . . . 70

3.3 Fisher Information of the Direction of Arrival Estimate . . . . . . . 79

3.4 Bayesian Information of the Direction of Arrival Estimate . . . . . . 82

3.5 Partition Optimization .................... 84

3.6 Results ....................... 86

3.6.1 Beamformer Optimization Results . . . . . . . . 86

3.6.2 Partition Optimization Results ........... 91

3.7 Conclusion . . . . . . . . . . . . . . . . 97

4 Adaptive Beamforming and Mutual Coupling Estimation Techniques for Aperture-Level Simultaneous Transmit and Receive Phased Arrays ...................... 101

4.1 Introduction . . . . . . . . . . . . . . . . . . 102

4.1.1 Algorithms for Channel Estimation and Adaptive Beamforming ......................... 104

4.1.2 Our Previous Work . . . . . . . . . . . . 107

4.1 .3 Outline ........................ 108 
4.1.4 Notation .................... 109

4.2 ALSTAR Array Signal Model . . . . . . . . . . . . . . . . . . . 110

4.2.1 Digital SIC in ALSTAR ............... 113

4.2 .2 Effective Isotropic Isolation . . . . . . . . . . . 117

4.3 Mutual Coupling Channel Estimation . . . . . . . . . . . . . 121

4.3.1 SMI Channel Estimation .............. 121

4.3.2 LMS Channel Estimation . . . . . . . . . . . . . 123

4.3.3 RLS Channel Estimation ............... 124

4.3.4 Performance Comparison Results . . . . . . . . . . . 125

4.4 Adaptive Beamforming for ALSTAR . . . . . . . . . . . . . 130

4.4.1 SMI Receive Beamformer \& Wiener Optimal Transmit Beam-

former .................... 130

4.4.2 LMS LCMV Receive Beamformer . . . . . . . . . . . . . 135

4.4.3 RLS LCMV Receive Beamformer . . . . . . . . . . . . . 136

4.4.4 Performance Comparison Results ............. 137

4.5 Conclusion . . . . . . . . . . . . . . . . 144

5 Conclusion ........................ 147

5.1 Future Work . . . . . . . . . . . . . . . . . . . 149

References ........................ 151

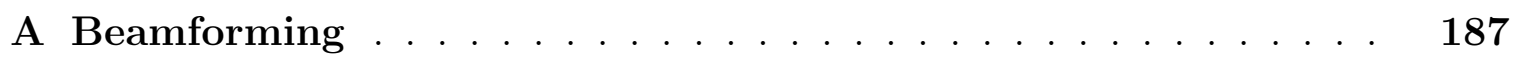


B The Cramér-Rao Lower Bound and The Bayesian Cramér-Rao

Lower Bound . . . . . . . . . . . . . . . . . . . . . . . . . . 193

B.1 Intuitive Derivation of the CRLB $\ldots \ldots \ldots \ldots \ldots$

B.1.1 CRLB Example . . . . . . . . . . . . . . . . . . . 197

B.2 Intuitive Derivation of the BCRB $\ldots \ldots \ldots \ldots$

B.2.1 BCRB Example $\ldots \ldots \ldots \ldots \ldots$

C Genetic Algorithms ................... 203

D Letters of Permission . . . . . . . . . . . . . . . . 207 



\section{List of Figures}

2.1 Example configurations of a $5 \times 10$ ALSTAR array, with transmitting elements black and receiving elements gray. Elements are physically identical and can be dynamically reassigned depending on overall sys-

tem requirements. . . . . . . . . . . . . . 25

(a) Partition A ................... 25

(b) Partition B . . . . . . . . . . . . 25

2.2 (a) Signal flow diagram of the ALSTAR phased array with multichannel observation receivers providing access to the distorted and noisy transmitted signals for SIC [1]. (b) Table of symbols and corre-

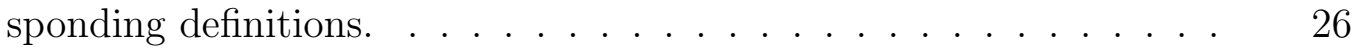

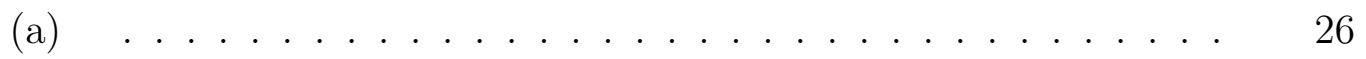

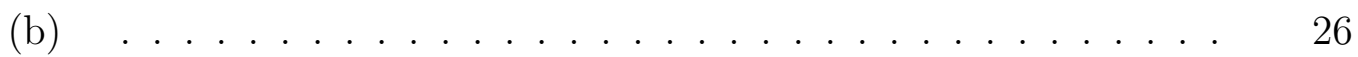

2.3 Signal flow diagram of a digital phased array with signal-only digital

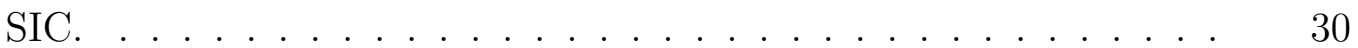


2.4 Example front end of a $T / R$ module that supports ALSTAR signal, noise, and distortion cancellation. In transmit mode (shown) the receiver measures the transmitted signal and noise through an attenuator. In receive mode (arrows) the receiver is connected to the antenna through a low noise amplifier and the transmitter is disabled [1]. . .

2.5 The singular value spectra of the coupling matrix $\mathbf{M}$ simulated in Ansys HFSS for the configurations shown in Fig. 2.1(a) and Fig. 2.1(b), respectively. . . . . . . . . . . . . . .

2.6 Effective Isotropic Isolation for the ALSTAR array configuration of Fig. 2.1(a), plotted across azimuth beam angle for non-adaptive beamformers without SIC at $2500 \mathrm{~W}$, non-adaptive beamformers with SIC at $2500 \mathrm{~W}$, and adaptive beamformers with SIC at $2.5 \mathrm{~W}, 25 \mathrm{~W}, 250$ $\mathrm{W}$, and $2500 \mathrm{~W}$ of total transmit power. The median EII for the adaptive beamformers with SIC was calculated across 100 initializations with randomly generated beamformers and one initialization with the non-adaptive beamformers. The error bars indicate the minimum and maximum EII achieved across all initializations. . . . . . . . . . . 
2.7 Noise power $P_{n}$ in the received signal for the ALSTAR array configuration of Fig. 2.1(a), plotted across azimuthal scan angle. The median $P_{n}$ for the adaptive beamformers with SIC is plotted with error bars to indicate the minimum and maximum $P_{n}$ achieved across all initializations. . . . . . . . . . . . . . . . .

2.8 (a) Effective Isotropic Radiated Power (EIRP) and (b) Receive Gain $\left(G_{r}\right)$ plotted against azimuth beam angle for the non-adaptive and adaptive beamformers for the ALSTAR array configuration of Fig. 2.1(a), The adaptive results show the median EIRP and $G_{r}$ with error bars indicating the minimum and maximum values across initializations. . . . . . . . . . . . . . . .

(a) EIRP vs. Azimuth Scan Angle . . . . . . . . . . . 53 53

(b) $G_{r}$ vs. Azimuth Scan Angle ...............

2.9 EII for the ALSTAR array configuration of Fig. 2.1(a), plotted across the scan space out to $60^{\circ}$ off broadside for the adaptive beamformers at $2.5 \mathrm{~W}$ transmit power. The initial condition for beamformer optimization was $\mathbf{b}_{t}=\mathbf{b}_{t, n a}$ and $\mathbf{b}_{r}=\mathbf{b}_{r, n a} \ldots \ldots \ldots$

2.10 EII for the ALSTAR array configuration of Fig. 2.1(a), plotted across the scan space out to $60^{\circ}$ off broadside for the adaptive beamformers at $2500 \mathrm{~W}$ transmit power. The initial condition for beamformer optimization was $\mathbf{b}_{t}=\mathbf{b}_{t, n a}$ and $\mathbf{b}_{r}=\mathbf{b}_{r, n a} \ldots \ldots \ldots$ 
2.11 Comparison of the transmitted power at each element and the incident power at each receive element between (a) the non-adaptive transmit beamformer and (b) the adaptive transmit beamformer for the aperture partition shown in Fig. 2.1(b) with $P_{t}=2500$ W. Significant reduction in receive element incident power was achieved via the adaptive transmit beamformer. . . . . . . . . . . . . . . 56

(a) Tx/Rx Element Power - Non-Adaptive Tx Beamformer . . . . 56

(b) Tx/Rx Element Power - Adaptive Tx Beamformer . . . . . 56

2.12 Effective Isotropic Isolation for the ALSTAR array configuration of Fig. 2.1(b), plotted across azimuth beam angle for non-adaptive beamformers without SIC at $2500 \mathrm{~W}$, non-adaptive beamformers with SIC at $2500 \mathrm{~W}$, and adaptive beamformers with SIC at $2.5 \mathrm{~W}, 25 \mathrm{~W}, 250$ $\mathrm{W}$, and $2500 \mathrm{~W}$ of total transmit power. The median EII for the adaptive beamformers with SIC was calculated over 100 initializations with randomly generated beamformers and one initialization with the non-adaptive beamformers. The error bars indicate the minimum and maximum EII achieved across all initializations. . . . . . . . . . 
2.13 Noise power $P_{n}$ in the received signal for the ALSTAR array configuration of Fig. 2.1(b), plotted across azimuthal scan angle. The median $P_{n}$ for the adaptive beamformers with SIC is plotted with error bars to indicate the minimum and maximum $P_{n}$ achieved across all initial-

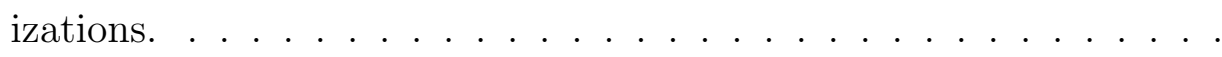

2.14 (a) Effective Isotropic Radiated Power (EIRP) and (b) Receive Gain $\left(G_{r}\right)$ plotted against azimuth beam angle for the non-adaptive and adaptive beamformers for the ALSTAR array configuration of Fig. 2.1(b), The adaptive results show the median EIRP and $G_{r}$ with error bars indicating the minimum and maximum values across initial-

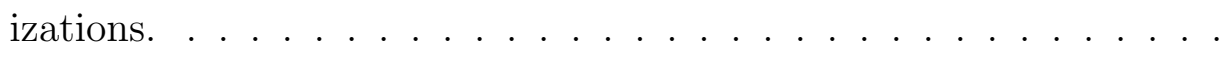

(a) EIRP vs. Azimuth Scan Angle . . . . . . . . . . . . 59

(b) $G_{r}$ vs. Azimuth Scan Angle $\ldots \ldots \ldots \ldots \ldots$

2.15 Comparison of the transmitted power at each element and the incident power at each receive element between (a) the non-adaptive transmit beamformer and (b) the adaptive transmit beamformer for the aperture partition shown in Fig. 2.1(b) with $P_{t}=2500$ W. . . . . 60

(a) $\mathrm{Tx} / \mathrm{Rx}$ Element Power - Non-Adaptive Tx Beamformer . . . 60 60

(b) $\mathrm{Tx} / \mathrm{Rx}$ Element Power - Adaptive Tx Beamformer ..... 60 
2.16 (a) Total Array Gain versus EII and (b) Total Array Gain versus ENF, at $\theta=30^{\circ}$ for the array configuration of Fig. 2.1(a), at $P_{t}=2.5 \mathrm{~W}, 25$ $\mathrm{W}, 250 \mathrm{~W}$, and $2500 \mathrm{~W}$. The regularization coefficient $\gamma$ varied from

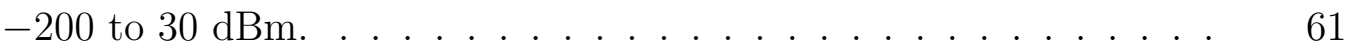

(a) Total Array Gain vs. EII . . . . . . . . . . . . . . . . 61

(b) Total Array Gain vs. ENF . . . . . . . . . . . . . 61 61

3.1 (a) List of symbols and their corresponding definitions. (b) ALSTAR architecture signal flow diagram, both from [1] and Chapter 2, . . 71]

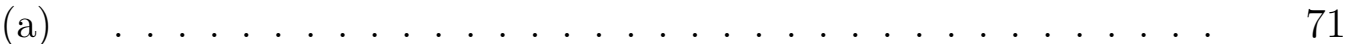

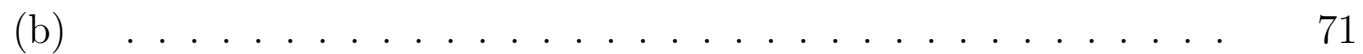

3.2 Definition of the coordinate system used for element positions and beam scan angles in this work. . . . . . . . . . . . . 73

3.3 Diagram of the switching system behind each element in an ALSTAR array [1]. When configured as shown, the antenna is connected to the transmit RF chain and the receiver is connected to a reference measurement tap near the antenna through an attenuator. In this way, the typically unused receiver is used to observe the transmitted signal and noise when the antenna is transmitting. When configured to receive, the switches connect the antenna to the receive $\mathrm{RF}$ chain via a low-noise amplifier. . . . . . . . . . . . . . . . 
3.4 This figure illustrates the operation of the genetic algorithm, modified from $[2 . \ldots \ldots \ldots \ldots \ldots \ldots$

3.5 Fisher Information plotted at each scan angle for the adaptive beamformers given the aperture is in the default configuration, shown in Fig. 3.9. For the adaptive beamformers, the median Fisher Information across initializations is plotted with error bars indicating the minimum and maximum Fisher Information achieved. Results are presented for $P_{t}=2.5 \mathrm{~W}, 25 \mathrm{~W}, 250 \mathrm{~W}$, and $2500 \mathrm{~W} \ldots \ldots \ldots$

3.6 Noise power plotted at each scan angle for the adaptive and nonadaptive beamformers with the default aperture partition shown in Fig. 3.9. The median noise power across initializations is plotted for the adaptive beamformers with error bars indicating the minimum and maximum noise power achieved. Results are presented for $P_{t}=2.5 \mathrm{~W}$, $25 \mathrm{~W}, 250 \mathrm{~W}$, and $2500 \mathrm{~W} \ldots \ldots \ldots \ldots$ 
3.7 EII plotted across the azimuth scan angles for the 10-element array with the default aperture partition shown in Fig. 3.9. Results for both the non-adaptive and adaptive beamformers are presented. For the non-adaptive beamformers, the EII is almost exactly the same between transmit power levels because the effective isotropic radiated power (EIRP) scales linearly with $P_{t}$ and residual noise scales almost linearly with $P_{t}$. For the adaptive beamformers, the median EII is plotted with error bars indicating the maximum and minimum achieved across the one non-adaptive initialization and 10 random initializations of the optimization routine. While maximizing EII was not the objective, this plot shows that maximizing Fisher Information produces beamformers with high isolation that are practical for STAR. Results are presented for $P_{t}=2.5 \mathrm{~W}, 25 \mathrm{~W}, 250 \mathrm{~W}$, and $2500 \mathrm{~W}$. . . . . . . . .

3.8 Bayesian Information distribution of the possible valid transmit/receive partitions for the 10-element array. The performance of the default partition with adaptive beamformers is plotted as a vertical black dashed line, and the performance of the GA optimized partitions are plotted as vertical red dashed lines. Five trials of the GA optimization were run at each power level. . . . . . . . . . . . . . . 
3.9 Default and optimized transmit/receive aperture partitions for the 10element array. Black segments represent transmitting antennas and white segments represent receiving antennas. Results are presented for $P_{t}=2.5 \mathrm{~W}, 25 \mathrm{~W}, 250 \mathrm{~W}$, and $2500 \mathrm{~W}$. The GA was run 5 times at each power level. All the trials produced the partition shown below or the equivalent partition with transmit and receive elements swapped.

3.10 Bayesian Information plotted for the default and GA optimized partitions for the $5 \times 10$-element array at $P_{t}=2.5 \mathrm{~W}, 25 \mathrm{~W}, 250 \mathrm{~W}$, and $2500 \mathrm{~W} \ldots \ldots \ldots \ldots \ldots \ldots \ldots \ldots \ldots \ldots$

3.11 Default and Optimized Partitions returned by the GA for the $5 \times 10$ element array. Black segments represent transmitting antennas and white segments represent receiving antennas. The optimized partitions presented here are the best out of 5 GA runs in terms of Bayesian

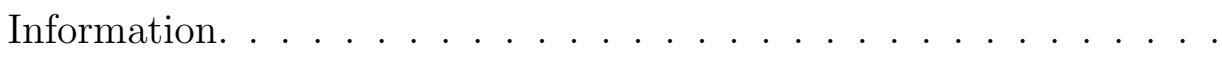

3.12 Fisher Information $\mathcal{J}(\theta)$ plotted against azimuth scan angle for the default and optimized partitions of the $5 \times 10$-element array at $P_{t}=2.5$ $\mathrm{W}$ and $2500 \mathrm{~W} \ldots \ldots \ldots \ldots \ldots \ldots \ldots$

3.13 Receiver noise power $\sigma_{n}^{2}$ plotted against azimuth scan angle for the default and optimized partitions of the $5 \times 10$-element array at $P_{t}=2.5$ $\mathrm{W}$ and $2500 \mathrm{~W} \ldots \ldots \ldots \ldots \ldots \ldots \ldots \ldots \ldots$ 
3.14 Number of unique transmit/receive partitions considered by the GA for both array sizes at each power level across 5 GA trials. The number of partitions that would be considered in an exhaustive search is also plotted for comparison. . . . . . . . . . . . . . . .

4.1 (a) ALSTAR digital phased array architecture signal flow diagram, including digital SIC, (b) Symbols and corresponding definitions for the ALSTAR architecture, modified from [1] and Chapter 2. . . . 111

4.2 Schematic of the switching system that enables each antenna to transmit or receive. When configured to transmit, the antenna is connected to the transmit RF front end via a power amplifier, and the transmitted signal, noise, and distortion are measured by the otherwise unused receive hardware. The receive channel is coupled to the reference tap on the antenna through a fixed attenuator in order to prevent receiver saturation or damage. When configured to receive (arrows), the receiver is coupled to the antenna through a low-noise amplifier, and the transmit channel is disconnected [1]. . . . . . . . . . . . 114

4.3 Outline of the operation of the channel estimation and adaptive beamforming techniques proposed in this work. . . . . . . . . . . 125 
4.4 Layout of the ALSTAR array simulated in this work. Black and grey elements represent transmitting and receiving antennas, respectively. Taken from Chapter 2 . . . . . . . . . . . . . . 126

4.5 Results of the convergence performance experiment for the LMS and RLS algorithms using the optimal parameters identified in the parameter searches. The final squared error of the SMI method is included for comparison. The minimum, mean, and maximum squared error of the channel estimate over the 1000 trials is plotted. . . . . . . . .

4.6 (a) Average EII and (b) $P_{i+n}$ at broadside plotted across 5 blocks for SMI, LMS, and RLS adaptive receive beamforming, respectively. The adaptive transmit beamformer in (4.41) was used with all the adaptive receive beamforming methods. The EII achieved at broadside by the ideal narrowband (NB) beamforming technique described in Chapter 2 and the thermal noise power $\sigma_{r}^{2}$ are plotted for comparison. . . . 139

4.7 (a) Average EII and (b) $P_{i+n}$ plotted across 101 evenly spaced azimuth scan angles $(\phi=0$ and $\theta \in[-\pi / 3, \pi / 3])$ for the SMI, LMS, and RLS receive beamformers in concert with the Wiener optimal transmit beamformer. External interference was absent from these trials. 10 trials were conducted at each scan angle. . . . . . . . . . . 141 
4.8 (a) Average EII and (b) $P_{i+n}$ plotted across 101 evenly spaced azimuth scan angles $(\phi=0$ and $\theta \in[-\pi / 3, \pi / 3])$ for the SMI, LMS, and RLS receive beamformers in concert with the Wiener optimal transmit beamformer. External interference was present in these trials. 10 trials were conducted at each scan angle. . . . . . . . . . . . 142

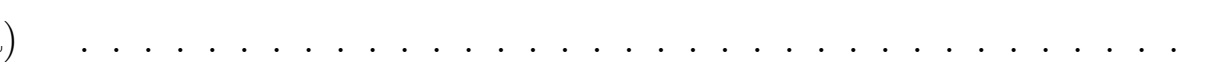

4.9 Computational complexity of the solutions based on the (a) SMI, (b) LMS and (c) RLS adaptive receive beamforming algorithms. The total computational complexity is given and it is also broken down into receive beamforming, transmit beamforming, and SIC complexities. For reference, the maximum computational output of a single Intel Stratix 10 FPGA and the Summit supercomputer at Oak Ridge National Laboratory are also plotted. . . . . . . . . . . . . .

A.1 Geometry of the basic beamforming problem for a uniform linear ar-

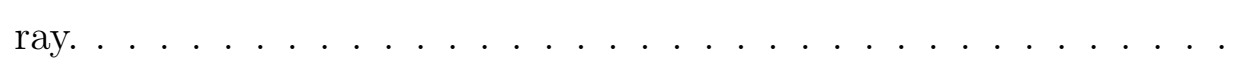

C.1 Illustration of the key elements of the GA. Figure taken from Chapter

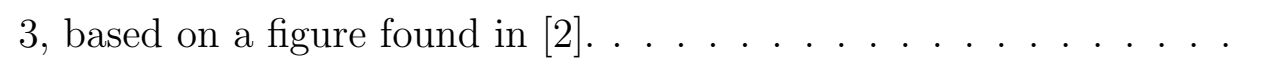




\section{List of Tables}

2.1 Acronyms $\ldots \ldots \ldots \ldots \ldots$

4.1 Channel Estimation Algorithm Computational Complexity . . . . 129 



\section{Preface}

\subsection{Chapter 2}

2020 IEEE. Reprinted, with permission, from

I.T. Cummings, J.P. Doane, T.J. Schulz, and T.C. Havens. Aperture-level simultaneous transmit and receive with digital phased arrays. Submitted to IEEE Trans. Signal Process..

Chapter 2 is a reproduction of the above work, on which I was the lead author. The theoretical work for this paper was begun by Dr. Doane, but I carried the paper to completion by implementing the proposed algorithms in code, investigating the practical difficulties with the proposed methods, contributing the regularization technique presented in the last section, and authoring the bulk of the manuscript.

\subsection{Chapter 3}

I.T. Cummings, T.J. Schulz, J.P. Doane, and T.C. Havens. Narrowband Direction of Arrival Estimation with Aperture-Level Simultaneous Transmit and Receive Digital 
Phased Arrays. In Preparation.

I.T. Cummings, T.J. Schulz, J.P. Doane and T.C. Havens, "An InformationTheoretic Approach to Partitioning Simultaneous Transmit and Receive Digital Phased Arrays," 2018 IEEE Radar Conference (RadarConf18), Oklahoma City, OK, 2018, pp. 0478-0483.

I.T. Cummings, T.J. Schulz, J.P. Doane, and T.C. Havens, Optimizing the Information-Theoretic Partitioning of Simultaneous Transmit and Receive Phased Arrays, in 2018 IEEE Int. Symp. Antennas and Propagation, Boston, MA, 2018.

Chapter 3 presents the first paper cited above, on which I was the lead author. The Cramér-Rao Lower Bound and Bayesian Cramér-Rao Lower Bound aspects of this paper were initiated by Dr. Schulz, but developed jointly between himself and myself. I introduced the idea of applying a Genetic Algorithm to the aperture partitioning problem. I also developed the simulations and wrote the manuscript. The first paper is an extension of the second and third papers cited above. For both of these papers I was the lead author, simulation developer, and manuscript writer. The theoretical foundations for these papers came from discussions between myself, Dr. Schulz, and Dr. Havens. 


\subsection{Chapter 4}

I.T. Cummings, J.P. Doane, T.J. Schulz, and T.C. Havens. Adaptive Beamforming and Mutual Coupling Estimation Techniques for Aperture-Level Simultaneous Transmit and Receive Phased Arrays. In Preparation.

Chapter 4 presents the paper cited above, for which I was the lead author. Broadly, the techniques presented in the work are the result of collaboration between Dr. Doane and myself. Adapting those techniques for use in this particular situation, implementing and debugging the algorithms, developing and running the required simulations and authoring the manuscript were my responsibility. 



\section{Acknowledgments}

First, I would like to thank my advisors, Dr. Tim Havens and Dr. Tim Schulz, for their kind and patient instruction over the years we have worked together. They taught me not only the technical skills expected of someone with a Ph.D. in Electrical Engineering, but also the softer skills of technical communication and professional relationships.

A big thank you also goes to Dr. Jon Doane of MIT Lincoln Laboratory (MITLL) for giving me the opportunity to work on such an exciting and meaningful project. He brought a practical mindset to my education and I am grateful that Jon continuously insisted on academic rigor in my work, scientific thought, and technical communication in a way that honed my skills as an engineer and scientist. Thank you as well to Dr. Jeff Herd, Dr. Brad Perry, Dr. Frank Robey, Ken Kolodziej, and the many other colleagues who made my time at MITLL so productive, stimulating, and enjoyable.

Dr. Eric Flynn and Dr. Adam Wachtor of Los Alamos National Laboratory (LANL) deserve a large measure of credit for convincing me to pursue a $\mathrm{Ph}$. D. in the first place. Alongside the rest of the staff at LANL's Engineering Institute, they showed me how exciting and engaging research could be, and in so doing convinced me that a Ph.D. was worth the effort. Together with Dr. Chuck Farrar, they were a huge 
part of the reason I received the NSF Graduate Research Fellowship, which made my collaboration with MITLL possible.

Dr. Gowtham also deserves sincere thanks. Not only did his Scientific Computing course make me a much more productive student and researcher, it taught me some of the finer points of preparedness and presentation in the field of research. Even more, Dr. Gowtham's mentorship continued beyond the classroom throughout my graduate studies, and he worked hard to make sure I had access to the computational tools I needed. Without his teaching and efforts, the work presented in this dissertation would not have been completed in the time frame that it was.

I would like to thank Kevin Gregory, who mentored me during my first engineering internships at Electro-Optics Technology, Inc. in the summers between my later high school years and after my first year at MTU. It was under his guidance that I learned how to work as an engineer at a company. His trust and confidence in my abilities pushed me to do my best, and it was his recommendation that opened the doors that led me on this path.

Finally, thank you to the many friends and colleagues for the times we got ate together, laughed together, and adventured together. These things made graduate school the experience it was and helped keep me going. Thank you.

This material is based upon work supported by the National Science Foundation 
Graduate Research Fellowship Program under Grant No. 1546592. Any opinions, findings, and conclusions or recommendations expressed in this material are those of the author(s) and do not necessarily reflect the views of the National Science Foundation.

This material is based upon work supported by the Under Secretary of Defense for Research and Engineering under Air Force Contract No. FA8721-05-C-0002 and/or FA8702-15-D-0001. Any opinions, findings, conclusions or recommendations expressed in this material are those of the author(s) and do not necessarily reflect the views of the Under Secretary of Defense for Research and Engineering.

Portage, a high-performance computing infrastructure at Michigan Technological University, was used in obtaining results presented in this publication. 



\section{List of Abbreviations}

\begin{tabular}{|c|c|}
\hline $\mathrm{ADC}$ & Analog-to-Digital Converter \\
\hline ALSTAR & Aperture-Level Simultaneous Transmit and Receive \\
\hline AWGN & Additive White Gaussian Noise \\
\hline $\mathrm{BCRB}$ & Bayesian Cramér-Rao Lower Bound \\
\hline $\mathrm{BF}$ & Beamformer \\
\hline $\mathrm{CDF}$ & Cumulative Distribution Function \\
\hline CRLB & Cramér-Rao Lower Bound \\
\hline CW & Continuous Wave \\
\hline $\mathrm{DAC}$ & Digital-to-Analog Converter \\
\hline DOA & Direction of Arrival \\
\hline DOF & Degrees of Freedom \\
\hline EII & Effective Isotropic Isolation \\
\hline EIRP & Effective Isotropic Radiated Power \\
\hline EIS & Effective Isotropic Sensitivity \\
\hline EM & Expectation Maximization \\
\hline ENF & Effective Noise Figure \\
\hline ESPRIT & Estimation of Signal Parameters via Rotational Invariance Techniques \\
\hline EZZB & Extended Ziv-Zakai Bound \\
\hline
\end{tabular}




$\begin{array}{ll}\text { FD } & \text { Full-Duplex } \\ \text { FIR } & \text { Finite Impulse Response } \\ \text { FLOPs } & \text { Floating Point OPerations } \\ \text { FLOPS } & \text { Floating Point OPerations per Second } \\ \text { FMCW } & \text { Frequency Modulated Continuous Wave } \\ \text { FPGA } & \text { Field-Programmable Gate Array } \\ \text { GA } & \text { Genetic Algorithm } \\ \text { IBFD } & \text { In-Band Full-Duplex } \\ \text { LMS } & \text { Least Mean Squares } \\ \text { LCMV } & \text { Linearly Constrained Minimum Variance } \\ \text { MAP } & \text { Maximum A Posteriori } \\ \text { MIE } & \text { Method of Interval Estimation } \\ \text { MIMO } & \text { Multiple-Input Multiple-Output } \\ \text { ML } & \text { Maximum Likelihood } \\ \text { MSE } & \text { Mean-Squared-Error } \\ \text { MSN } & \text { Maximum Signal-to-Noise } \\ \text { MUSIC } & \text { MUltiple SIgnal Classification } \\ \text { NB } & \text { Narrowband } \\ \text { RPSK } & \text { Quadrature Phase Shift Keyed } \\ \text { RFS } & \text { Radio Frequency } \\ \text { Recursive Least Squares }\end{array}$




$\begin{array}{ll}\text { SI } & \text { Self-Interference } \\ \text { SIC } & \text { Self-Interference Cancellation } \\ \text { SINR } & \text { Signal-to-Interference-Plus-Noise Ratio } \\ \text { SLNR } & \text { Signal-to-Leakage-Plus-Noise Ratio } \\ \text { SMI } & \text { Sample Matrix Inversion } \\ \text { SNR } & \text { Signal-to-Noise Ratio } \\ \text { SoI } & \text { Signal of Interest } \\ \text { STAR } & \text { Simultaneous Transmit and Receive } \\ \text { T/R } & \text { Transmit/Receive } \\ \text { ULA } & \text { Uniform Linear Array } \\ \text { WWB } & \text { Weiss-Weinstein Bound }\end{array}$





\section{Abstract}

In the signal processing community, it has long been assumed that transmitting and receiving useful signals at the same time in the same frequency band at the same physical location was impossible. A number of insights in antenna design, analog hardware, and digital signal processing have allowed researchers to achieve simultaneous transmit and receive (STAR) capability, sometimes also referred to as in-band full-duplex (IBFD).

All STAR systems must mitigate the interference in the receive channel caused by the signals emitted by the system. This poses a significant challenge because of the immense disparity in the power of the transmitted and received signals. As an analogy, imagine a person that wanted to be able to hear a whisper from across the room while screaming at the top of their lungs. The sound of their own voice would completely drown out the whisper.

Approaches to increasing the isolation between the transmit and receive channels of a system attempt to successively reduce the magnitude of the transmitted interference at various points in the received signal processing chain. Many researchers believe that STAR cannot be achieved practically without some combination of modified antennas, analog self-interference cancellation hardware, digital adaptive beamforming, 
and digital self-interference cancellation. The aperture-level simultaneous transmit and receive (ALSTAR) paradigm confronts that assumption by creating isolation between transmit and receive subarrays in a phased array using only digital adaptive transmit and receive beamforming and digital self-interference cancellation.

This dissertation explores the boundaries of performance for the ALSTAR architecture both in terms of isolation and in terms of spatial imaging resolution. It also makes significant strides towards practical ALSTAR implementation by determining the performance capabilities and computational costs of an adaptive beamforming and self-interference cancellation implementation inspired by the mathematical structure of the isolation performance limits and designed for real-time operation. 


\section{Chapter 1}

\section{Introduction}

Over the last several decades, a great deal of research effort has been directed at achieving the goal of transmitting and receiving meaningful signals from the same physical location, in the same frequency band, at the same time. This technology is often referred to as simultaneous transmit and receive (STAR) or in-band fullduplex (IBFD) operation, and it has been pursued so heavily because it offers a number of advantages and capabilities that address critical needs and shortcomings in a wide range of wireless sensing and communications tasks. Perhaps the most frequently cited benefit of STAR is that it could potentially double the bandwidth efficiency of communications links by allowing a single band to be allocated for uplink and downlink. Additionally, STAR could reduce the overhead required by some time-division multiplexing arbitration schemes. STAR also makes interesting radar 
paradigms possible. While continuous wave $(\mathrm{CW})$ and frequency-modulated continuous wave (FMCW) radars have been transmitting and receiving simultaneously in the same frequency band for quite some time, they have done so with limited system flexibility and high costs in terms of size, weight, and power. Achieving STAR in a radar platform could enable continuous target illumination during sensing and significantly reduce a radar's visibility to other sensors by allowing for long-period illumination with low-power waveforms.

\subsection{Problem Context}

The key challenge in successfully implementing STAR is mitigating the interference and noise that appears in the received signal due to the co-located transmitter. If all of the components in the system were noiseless and we could perfectly estimate the coupling from the transmit channel to the receive channel, then the interference component could simply be subtracted out. However, real components do have noise, so the simple subtraction approach will not perfectly cancel all the interference. Additionally, the extreme difference in transmitted and received signal power is often on the order of $100 \mathrm{~dB}$ [3], which means that the limited dynamic range of physical components becomes a significant factor.

The many techniques for isolating the transmit and receive channels from one another 
are best classified by the domain in which they operate: the electromagnetic domain, the analog domain, or the digital domain. The electromagnetic domain encompasses approaches that use the properties of electromagnetic wave propagation through solid media and free space to achieve transmit/receive isolation. This includes transmitting and receiving on opposite polarizations, building antennas and antenna arrays with reduced mutual coupling between transmit and receive antennas or ports, and using ferromagnetic circulators to partially isolate the transmitted and received signals within a single antenna. However, electromagnetic domain approaches are often bandwidth limited, bulky, heavy, or work well only for a particular application. Analog domain approaches fall between the antenna ports and the analog-to-digital or digitalto-analog converters (ADCs and DACs, respectively). In general, these approaches couple the transmitted signal and noise through some sort of filter and add it to the received signal to cancel out self-interference. However, analog domain approaches are often power intensive, expensive, and physically large. Techniques in the digital domain include digital transmit and receive beamforming, digital self-interference cancellation, and nonlinear equalization. Digital transmit and receive beamforming in phased arrays can be used to place near-field nulls on the receiving and transmitting elements, respectively. Note that transmit beamforming is grouped with the digital techniques instead of the electromagnetic techniques because its effect is often used in a similar manner to receive beamforming within the context of STAR. Digital self-interference cancellation is similar to the analog domain approaches in that it 
uses either the ideal transmitted signal or a measured copy of the transmitted signal and noise and an estimate of the transmit/receive coupling channel to approximate and cancel out the self-interference in the receive channel. Nonlinear equalization attempts to digitally pre-distort the transmitted signal in order to reduce the nonlinear effects of the power amplifier. Digital approaches are constrained by the fact that ADCs and DACs have limited dynamic range, and so care must be taken to avoid burying the received signal of interest beneath the receiver noise after all noise, interference, and cancellation effects are taken into account. A number of researchers have used techniques from more than one domain at the same time in order to achieve higher isolation than was possible with techniques from just one domain. A number of examples of techniques from all three domains, as well as examples of cross-domain approaches can be found in Section 2.1. Additionally, [3, 4, 5] provide broad surveys of the state of the art across the domains.

This dissertation studies the Aperture-Level STAR (ALSTAR) digital phased array architecture originally proposed in [1] and demonstrated in [6]. The approach is referred to as ALSTAR because it seeks to create isolation between the transmit and receive apertures of a single phased array. It leverages adaptive digital transmit and receive beamforming as well as digital self-interference cancellation to achieve high transmit/receive isolation, which places this approach within the digital domain. Remaining within the digital domain allows the ALSTAR architecture to support a 
wide number of communications and sensing tasks while remaining lightweight, lowcost, and space efficient.

\subsection{Dissertation Outline and Contributions}

\subsubsection{Chapter 2}

Chapter 2 describes the ALSTAR digital phased array architecture in detail and proposes a novel method of optimizing the transmit and receive beamformers in order to maximize the effective isotropic isolation (EII) achieved by the array. EII is calculated and the beamformers are optimized based on a sophisticated statistical signal model of the array that accounts for the limited dynamic range of the transmit and receive channels and the mutual coupling between them. Results are also presented for regularized beamforming techniques that allow for trade-offs between EII and transmit/receive gain. 


\subsubsection{Chapter 3}

In Chapter 3, bounds for narrowband direction of arrival (DOA) estimation performance are presented for an ALSTAR array. Adaptive beamforming and transmit/receive aperture partition optimization methods are explored that seek to minimize those error bounds. Specifically, the Fisher Information of the estimated DOA is calculated given a statistical signal model similar to the one presented in Chapter 2. The Fisher Information is optimized in terms of the transmit and receive beamformers for each look angle using an iterative technique similar to the technique given in Chapter 2. The Bayesian Information (i.e. the Fisher Information averaged across scan points) is calculated as a performance metric for the transmit/receive aperture partitioning. The task of aperture partitioning is performed with a genetic algorithm, which is shown empirically to significantly reduce the required computation over exhaustive partition searching.

\subsubsection{Chapter 4}

While the adaptive beamforming algorithm presented in Chapter 2 promises excellent EII performance, it would be difficult to implement in real-time due to its high computational complexity and iterative nature. Furthermore, it is formulated for a 
narrowband signal model, it assumes perfect knowledge of the mutual coupling channel, and it is not designed to handle external sources of interference. Inspired by the structure of the adaptive beamforming technique presented in Chapter 2, the adaptive beamforming techniques presented in this work seek similar goals while operating only on the observable signals available on the array, only assuming an imperfect knowledge of the mutual coupling channel provided by a practical mutual coupling channel estimation technique, and with knowledge of the DOA of the signal of interest.

\subsubsection{Chapter 5}

Chapter 5 concludes this dissertation by discussing the implications of fully digital ALSTAR technology, and highlighting the contributions of this work in terms of theoretical and practical adaptive beamforming methods, DOA performance bounds, ALSTAR transmit/receive aperture partition optimization via genetic algorithm. Additionally, future directions of potential research based on this material will be explored. 


\subsection{List of Relevant Publications}

I.T. Cummings, T.J. Schulz, J.P. Doane and T.C. Havens, "An InformationTheoretic Approach to Partitioning Simultaneous Transmit and Receive Digital Phased Arrays," 2018 IEEE Radar Conference (RadarConf18), Oklahoma City, OK, 2018, pp. 0478-0483.

I.T. Cummings, T.J. Schulz, J.P. Doane, and T.C. Havens, Optimizing the Information-Theoretic Partitioning of Simultaneous Transmit and Receive Phased Arrays, in 2018 IEEE Int. Symp. Antennas and Propagation, Boston, MA, 2018.

I.T. Cummings, T.J. Schulz, J.P. Doane, S.A. Zekavat, and T.C. Havens, Information-Theoretic Optimization of Full-Duplex Communication between Digital Phased Arrays, in 56th Annual Allerton Conference on Communication, Control, and Computing, Monticello, IL, 2018.

I.T. Cummings, T.J. Schulz, T.C. Havens, and J.P. Doane. Neural networks for real-time adaptive beamforming in simultaneous transmit and receive digital phased arrays. Accepted to 2019 IEEE Int. Symp. On Phased Array Syst. and Technol. (PAST), Waltham, MA, USA, Oct 2019. 
I.T. Cummings, J.P. Doane, T.J. Schulz, and T.C. Havens. Aperture-level simultaneous transmit and receive with digital phased arrays. Submitted to IEEE Trans. Signal Process..

I.T. Cummings, T.J. Schulz, J.P. Doane, and T.C. Havens. Narrowband Direction of Arrival Estimation with Aperture-Level Simultaneous Transmit and Receive Digital Phased Arrays. In Preparation.

I.T. Cummings, J.P. Doane, T.J. Schulz, and T.C. Havens. Real-Time Adaptive Beamforming via Neural Networks in Simultaneous Transmit and Receive Digital Phased Arrays. In Preparation. 



\section{Chapter 2}

\section{Aperture-Level Simultaneous}

\section{Transmit and Receive with Digital}

\section{Phased Arrays}

\section{$2.1 \quad$ Introduction}

Recently, interest has increased in the possibility of transmitting and receiving at the same time over the same channel. Traditionally, multiple users access a single wireless channel by dividing the resources of that channel between the users [7]. Multiple 
access schemes can be broadly categorized as time-division multiple access, frequencydivision multiple access, code-division multiple access, spatial-division multiple access (e.g. beamforming), or some combination of several techniques. Each one makes trades in terms of complexity of implementation, bandwidth requirement, and continuity of access for any given user. This makes certain schemes more or less appropriate for specific applications. In this work we consider the problem of achieving in-band full-duplex (FD) operation with a digital phased array, also referred to as simultaneous transmit and receive (STAR). Many STAR applications involve non-cooperative users, and code-division multiple access schemes are therefore not considered. Nevertheless, the techniques developed here could be combined with other multiple access schemes, improving the overall throughput of a particular channel given a specific application. While it is natural to discuss STAR from a communications point of view, the techniques detailed in this paper enable applications in communications, radar, imaging, and multi-function systems.

The problem that must be solved in order to implement STAR is mitigating the selfinterference and mutual coupling between the transmit and receive channels. The mutual coupling is often strong enough to saturate receiver hardware if not properly handled. We classify the approaches to this challenge into three categories: electromagnetic, analog, and digital self-interference mitigation techniques. Electromagnetic techniques include the effort to design antennas and antenna arrays with high directionality, spatial nulls, or polarization diversity to enable STAR [8, 9, 10, 11, 12, 13, 
14, 15, 16, 17, 18, 19, 20, 21, 22, 23, 24, 25, 26, 27, 28, 29, 30, 31, 32, 33, 34, 35, 36, 37.

These antennas often suffer performance losses in phased arrays or require different polarizations on transmit and receive, limiting performance and relevance for many applications. Also included in electromagnetic techniques, ferrite circulators (and other non-ferrite circulators) can be used to create transmit/receive isolation within a single antenna [38, 39]. However, the isolation achieved is often limited and may have a high cost in terms of size and weight. Analog techniques include approaches that attempt to match and cancel the mutual coupling from transmit to receive channels by introducing analog cancellation filters into the front end of the transceiver [40, 41, 42, 43, 44, 45, 46, 47, 48, 49, 50, 51, 52. However, both electromagnetic and analog approaches often introduce significant costs to the system in terms of size, weight, complexity, and/or power consumption. This becomes especially important in the case of a phased array, because the number of self-interference channels to be canceled grows with the number of unique transmit/receive antenna pairs [53]. This can make solutions that involve fully-connected analog cancellation networks or bulky RF hardware near the antennas impractical, especially given the tight space constraints typical of phased arrays. Furthermore, analog cancellation hardware can introduce constraints on transmit power and new sources of receiver noise.

The ALSTAR digital phased array studied in this paper-first proposed in [1] and [6] - uses only digital self-interference mitigation techniques, which include adaptive digital beamforming and digital self-interference cancellation (SIC). It is important to 
note that the ALSTAR phased array architecture retains the operational capabilities of a fully-digital phased array and does not rely on analog cancellation techniques that increase array cost and complexity and reduce power efficiency. While both [3] and [5] mention adaptive transmit and receive beamforming and digital SIC as viable self-interference reduction methods, they both assume that digital techniques must be complimented by additional electromagnetic and/or analog mitigation schemes, as in [53, 54, 55, 56, 57, 58, 59, 60, 61, 62, 63, 64, 65, 66, 67, 68, 69, 70, 71, 72, 73, 74, 75. However, [1] and [6] demonstrated that in the context of the ALSTAR architecture, digital methods may be sufficient. Where many approaches focus on canceling the signal component of the self-interference, the ALSTAR architecture cancels the signal, noise, and distortion components of the self-interference signal by implementing SIC with digital observations of the noisy and distorted transmit signal. The work presented in this paper improves upon [1] by exploiting the interdependence of the transmit and receive beamformers in creating isolation between transmit and receive channels with limited dynamic range, at the cost of a more complex adaptive beamforming process. The adaptive transmit beamformer creates nulls to reduce the incident transmitted signal and noise on the receivers in order to avoid receiver saturation, thereby lowering the noise floors of the limited dynamic range receive channels, while still achieving high transmit gain in the direction of interest. At the same time, the adaptive receive beamformer rejects the remaining interference and noise (after SIC) passing through the mutual coupling channel while maintaining 
relatively high gain in the direction of interest. The details of how the adaptive beamforming method achieves these goals can be found in Section 2.2.3 and Section 2.3. We present simulated results that suggest that very high isolation levels are possible across the scan space of the digital phased array being studied. We also discuss how effective isotropic isolation (EII) may be traded for transmit/receive gain via a regularization technique. The remaining discussion of previous work by others focuses on digital techniques aimed at solving similar problems. It should be noted that while many of the references discussed involve multiple-input multiple-output (MIMO) arrays, this work only considers single-input single-output phased arrays.

\subsubsection{Digital Adaptive Beamforming for STAR}

Forming near-field nulls on the receivers in an array is an effective method to reduce the effects of mutual coupling in phased arrays. In this way, adaptive digital beamforming can prevent the receivers from becoming saturated, making further digital SIC possible. Everett et al. demonstrated the use of digital transmit beamforming to reduce the self-interference present at the receive antennas in a MIMO array while reserving a specified number of multiplexing degrees of freedom (DOF) for the desired transmitted signals [76]. They explored the performance of their beamforming scheme in terms of FD sum-rate as a function of reserved DOF. Placing near-field nulls requires a good estimate of the mutual coupling channel in the phased array. To solve 
this problem, Johnston and Fiore developed a wideband MIMO least-mean-squares channel estimation procedure and used the resulting channel model in a null-space projection adaptive beamforming algorithm [77]. Masmoudi and Le-Ngoc developed a maximum-likelihood estimator for the mutual coupling in an FD MIMO system [78].

\subsubsection{Digital SIC for STAR}

In addition to adaptive beamforming, digital SIC can provide a significant increase in isolation between the transmit and receive channels. Ahmed and Eltawil demonstrated a single-channel digital SIC scheme using an auxiliary receive channel to measure the transmitted signal (including distortion and noise) [79]. Our work extends their proposed technique to a phased array system. The authors in [80] used an auxiliary receive channel to observe the distorted and noisy transmit signal, which they fed into an independent components analysis algorithm to isolate the desired received signal. Li et al. presented a digital SIC model that accounted for the non-ideal nature of the physical components in the transmit and receive signal processing chains [81]. Komatsu et al. explored a non-linear frequency-domain digital SIC technique [82]. Liang et al. presented an approach that implements adaptive transmit beamforming in concert with digital SIC [83]. Their signal-to-leakage-plus-noise-ratio (SLNR) based precoding technique is similar to the one proposed in this work. However, our 
objective function also accounts for the noise injected into the receive channel by the digital SIC process and the limited dynamic range of the transmit and receive channels. Furthermore, they do not consider the interaction between the transmit and receive beamformers.

\subsubsection{Advanced Digital Techniques for STAR}

A great deal of work has been done in developing optimal and practical transmit and receive beamforming schemes that mitigate self-interference in FD MIMO networks. Some works have explored minimizing the mean-squared-error (MSE) of the signals at the receivers in FD MIMO networks, accounting for interference [84, 85, 86, 87, 88, 89]. A number of papers have considered bit-rate maximization (e.g. sum-rate, weighted-sum-rate) methods for FD MIMO networks experiencing self-interference [90, 91, 92, 93, 94, 95, 96, 97, 98, 99, 100, 101]. Lin et al. present two methods for beamformer design in an amplify and forward FD MIMO relay scenario that minimize symbol vector error rate [102]. Still others have considered signal-to-interference-plusnoise ratio (SINR) or SLNR based objective functions or constraints [103, 104, 105, 106, 107, 108, 109]. Several authors have written about methods for beamforming in FD MIMO networks referred to as zero-forcing, null-space-projection, or interference alignment [110, 111, 112, 113, 114, 115, 116]. Interference alignment extends the concept of zero-forcing beamforming to include null-space constraints on inter-user 
interference.

The following discussion highlights the similarities and differences between the work presented in this paper and related previous works by other authors. Hwang et al. described an alternating optimization method for SINR maximization in amplify and forward MIMO relays by beamforming [117]. However, their SINR metric is structurally different than EII and their work assumed the use of analog SIC where we assume that only digital beamforming and SIC are implemented. Xiao et al. addressed the concept of interference mitigation via beamforming (in concert with other techniques) in FD millimeter wave communications scenarios, but they focused on constant-amplitude (i.e. phase-only) and zero-forcing beamforming techniques [118]. Xia et al. described an FD MIMO relay model that accounts for limited dynamic range in the transmit and receive channels [119]. They optimized the beamformers at the relay to minimize the self-interference power via a combined minimum eigenvalue and zero-forcing procedure, while assuming no SIC is performed. Shen et al. explored an iterative rate maximization approach for the beamformers in an FD MIMO one-way relay link (including the source and the destination) [120]. Their approach is similar to the one presented in this paper in that it models the limited dynamic range of the transmit and receive channels. However, their work does not consider the possibility of canceling the noise component of the self-interference as is done in this work. 
Riihonen et al. discussed the problem of mitigating loopback interference in FD MIMO relays [121]. They considered SIC and adaptive beamforming techniques separately and together. They also considered the distortion of the transmitted and received signals due to beamforming, and extended their minimum interference beamforming techniques to jointly minimize the MSE of the transmitted and received signals. However, the joint beamforming techniques that were presented reduce to separate optimizations that do not take advantage of the interdependence between transmit and receive beamformers.

Day et al. proposed a combination of pilot-based self-interference channel estimation, transmit and receive precoding, and digital SIC for FD MIMO applications [122]. Both [122] and this work model the effects of limited transmitter and receiver dynamic range and use both transmit and receive beamforming and SIC to mitigate self-interference. Day et al. optimized MIMO sum-rate across a pair of communicating nodes, which is a function of the link SINRs in both directions in the full-duplex link, whereas the EII objective function considered in this work describes the isolation between the transmit and receive channels in a single phased array system. Furthermore, because link SINR is quantified prior to receive beamforming in [122, the mutual interdependence of the transmit and receive beamformers was not exploited in maximizing the MIMO sum-rate. This work takes advantage of the beamformer interdependence in order to maximize the transmit/receive isolation in the ALSTAR phased array architecture. 
Antonio-Rodríguez et al. discussed a phased array system very similar to the ALSTAR architecture, operating as a decode-and-forward full-duplex relay [123]. They optimized the SINR at the relay after cancellation and receive beamforming, in terms of the relay transmit and receive beamformers. However, their optimization used constraints to obtain non-trivial transmit filters, where this work incorporates the transmit gain directly into the objective function. Furthermore, they decoupled their transmit filter optimization step from the receive filter optimization by suboptimally substituting the SINR incident on the relay as their transmit filter objective function. In a later paper, Antonio-Rodríguez et al. updated their approach by first minimizing the noise power at the relay after cancellation and receive filtering in terms of the transmit filter, while guaranteeing a minimum signal power at the destination via a null-space projection step in the transmit filter optimization [124. The receive filter optimization step remained unchanged, but they also iterated between the transmit and receive filter optimizations to allow for the coupled nature of the transmit and receive filter optimization problems. The main difference between [124] and this work is that we incorporate transmit gain into the EII metric instead of introducing a signal power constraint.

Guo and Wang presented an alternating optimization algorithm to find the transmit and receive beamformers that maximize the SINR at the destination in an FD MIMO link using either Dinkelbach's algorithm or a closed-form suboptimal solution at each iteration [125]. The alternating optimization of an SINR metric is similar to the 
approach presented in this paper. However, their SINR metric specifically describes an amplify and forward MIMO relay, and they included a recursive self-interference component not present in our model because we assume that the received signal of interest and self-interference signals are uncorrelated.

Taghizadeh et al. considered FD amplify and forward relays with a realistic model for transmit and receive RF hardware and a general model for SIC [126]. Additionally, their proposed AltMuStR1 algorithm and objective functions are similar to the ones presented in this work. However, their signal model and optimization techniques do not account for the effect of the coupled transmitter noise on the noise in the limited dynamic range receive channels. The work presented in this paper specifically models digital SIC and adaptive beamforming techniques that mitigate the transmitted noise component in addition to the transmitted signal component in the self-interference present at the receiver. Additionally, we present isolation results across the scan space of the array and a regularization method that allows for trade-offs between array gain and self-interference reduction, where [126] does not.

\subsubsection{Previous Work, Contributions, \& Organization}

The work presented in this paper is based on the ALSTAR architecture originally

presented in [1], where the EII metric was also presented. An 8-element uniform 
linear ALSTAR array was demonstrated in [6], achieving $140.5 \mathrm{~dB}$ of EII across a $100 \mathrm{MHz}$ bandwidth centered at $2.45 \mathrm{GHz}$. We simulated the performance of the ALSTAR architecture under a simplified signal model in a narrowband imaging scenario [127] and explored transmit/receive aperture partition optimization via genetic algorithm for the same architecture [128]. Additionally, we explored optimizing the beamformers and aperture partition in a narrowband FD communication link between two ALSTAR arrays, also with a simplified signal model [129].

In this paper, we calculate EII for a digital phased array with limited dynamic range transmit/receive channels and propose a novel method to optimize the transmit and receive beamformers to maximize EII. We then simulate the performance of the architecture and our proposed optimization method for a $5 \times 10$ ALSTAR array with two unique aperture partitions.

Section 2.2 describes the ALSTAR array architecture in detail and develops the corresponding statistical signal model. Section 2.3 motivates the definition of EII from a self-interference mitigation perspective, derives the EII metric for the signal model developed previously, and proposes an iterative technique to find the beamformers that maximize EII. While the work presented in this paper considers beamformer optimization after estimating the channels involved, an iterative adaptive method that updates both the estimated channel matrices and beamformers could be more practical and will be considered in future work. However, the methods presented in this 
Table 2.1

Acronyms

\begin{tabular}{cl} 
Acronym & Definition \\
\hline STAR & Simultaneous Transmit and Receive \\
ALSTAR & Aperture-Level Simultaneous Transmit and Receive \\
EII & Effective Isotropic Isolation \\
DOF & Degrees of Freedom \\
SIC & Self-Interference Cancellation \\
MIMO & Multiple-Input Multiple-Output \\
FD & Full-Duplex \\
SLNR & Signal-to-Leakage-Plus-Noise Ratio \\
MSE & Mean-Squared-Error \\
SINR & Signal-to-Interference-Plus-Noise Ratio \\
RF & Radio Frequency \\
SNR & Signal-to-Noise Ratio \\
AWGN & Additive White Gaussian Noise \\
EIRP & Effective Isotropic Radiated Power \\
EIS & Effective Isotropic Sensitivity \\
ENF & Effective Noise Figure \\
\hline
\end{tabular}

work highlight the factors that affect the performance of the ALSTAR architecture and illustrate its potential performance. Section 2.4 details the simulated performance of an ALSTAR array with two transmit/receive aperture partitions using the proposed optimization technique. Section 2.5 describes a regularization technique that makes it possible to trade EII for total array gain and presents results. Section 2.6 draws conclusions from the results of the simulation and discusses anticipated extensions and applications of the architecture, performance metric, and techniques discussed in this work. Table 2.1 lists the acronyms used in this work. 


\subsection{Aperture-Level Simultaneous Transmit and Receive (ALSTAR) Array}

The ALSTAR architecture is implemented on a fully digital array with digital transceivers at each element [130]. Rather than require each element in the array to simultaneously transmit and receive, we partition the aperture into a transmitting subarray and an adjacent receiving subarray, e.g., as in Fig. 2.1. We therefore refer to this approach as Aperture-Level Simultaneous Transmit and Receive (ALSTAR), to distinguish it from other architectures which might support STAR at the element level. All elements in the array are physically identical and can be dynamically assigned to either transmit or receive. The size and geometry of the transmit and receive subarrays can thus be dynamically modified to support various patterns and functions as needed by the overall system. In the degenerate case that all elements are transmitting or receiving, the array may operate in the typical (half-duplex) manner without performance degradation, which is an uncommon advantage of the ALSTAR architecture among STAR systems.

The architecture proposed in [1] for digital SIC in a phased array with $J$ transmitting elements and $K$ receiving elements is depicted in Fig. 2.2. In this work we consider a narrowband signal model for notational and analytical clarity, but the model could 


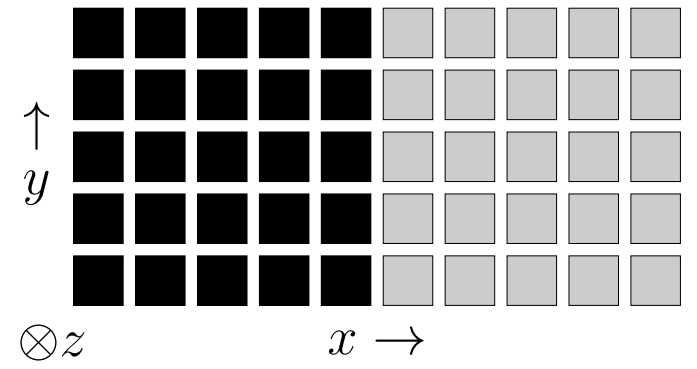

(a) Partition A

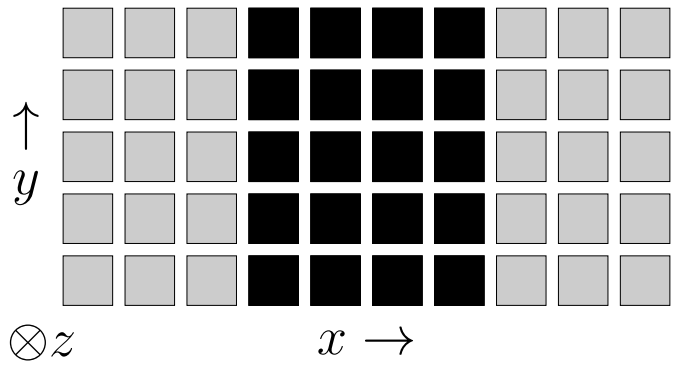

(b) Partition B

Figure 2.1: Example configurations of a $5 \times 10$ ALSTAR array, with transmitting elements black and receiving elements gray. Elements are physically identical and can be dynamically reassigned depending on overall system requirements.

be converted to a wideband model by extending the channel models, beamforming vectors, and SIC filter to multitap finite impulse response filters. The transmitted signal vector $\mathbf{t}(n) \in \mathbb{C}^{J \times 1}$ at time index $n$ is

$$
\mathbf{t}(n)=\mathbf{b}_{t} x(n)+\mathbf{n}_{t}(n),
$$

where $x(n)$ is the desired signal to be transmitted with $E\left[|x(n)|^{2}\right]=1, \mathbf{b}_{t} \in \mathbb{C}^{J \times 1}$ is the vector of transmit beamforming coefficients, and $\mathbf{n}_{t}(n)$ is zero-mean, complex additive white Gaussian noise (AWGN) transmitted noise with covariance matrix $\mathbf{N}_{t}=E\left[\mathbf{n}_{t} \mathbf{n}_{t}^{\mathrm{H}}\right]=\operatorname{Diag}\left(\mathbf{b}_{t} \mathbf{b}_{t}^{\mathrm{H}}\right) / \eta_{t}$ where $\eta_{t}$ is the signal-to-noise ratio (SNR) of each transmitter. The received signal vector $\mathbf{r}(n) \in \mathbb{C}^{K \times 1}$ is

$$
\mathbf{r}(n)=\mathbf{s}(n)+\mathbf{M t}(n),
$$

where $\mathbf{s}(n) \in \mathbb{C}^{K \times 1}$ represents the external signal(s) of interest, with $\mathbf{R}_{s s}=E\left[\mathbf{s s}^{\mathrm{H}}\right]$, 


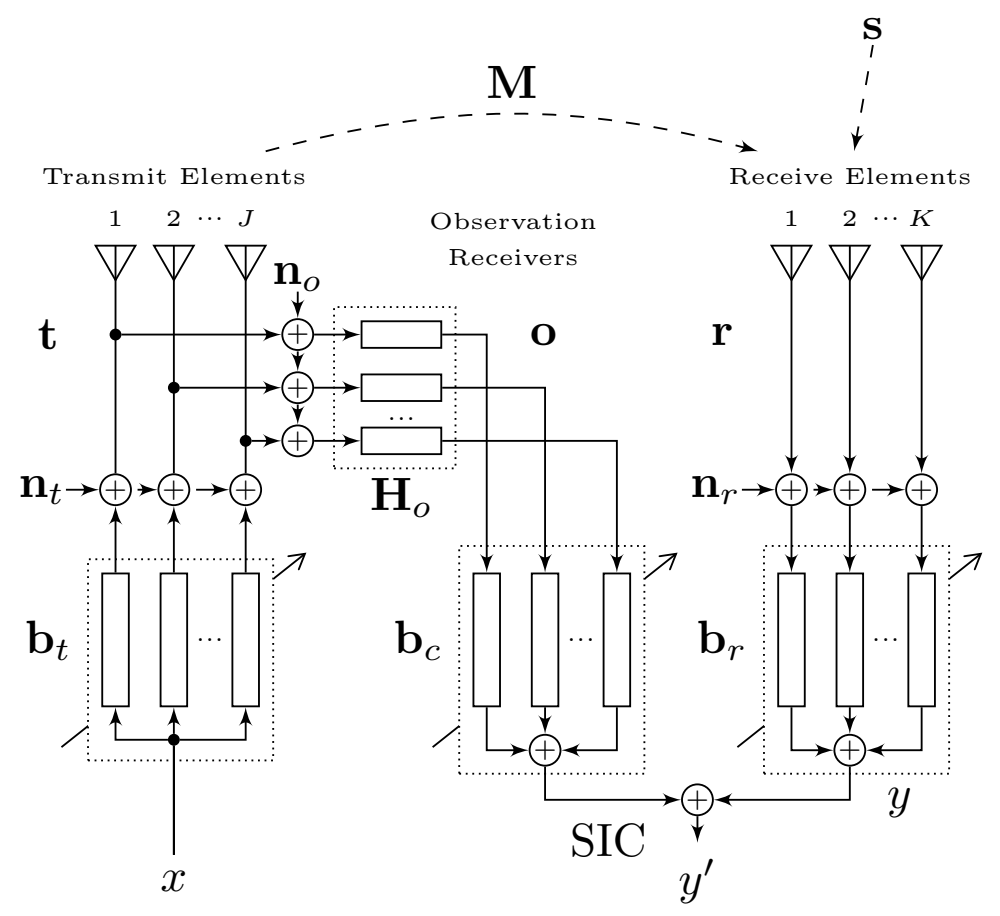

(a)

\begin{tabular}{cl} 
Symbol & Definition \\
\hline$x$ & Transmitted Signal \\
$\mathbf{b}_{t}$ & Transmit Beamformer \\
$\mathbf{n}_{t}$ & Transmitter Noise \\
$\mathbf{t}$ & Transmitted Signal + Noise \\
$\mathbf{n}_{o}$ & Observation Noise \\
$\mathbf{H}_{o}$ & Observation Channel \\
$\mathbf{o}$ & Observed Transmit Signals \\
$\mathbf{b}_{c}$ & Cancellation Filter \\
$\mathbf{M}$ & Mutual Coupling Channel \\
$\mathbf{s}$ & Signal of Interest \\
$\mathbf{n}_{r}$ & Receiver Noise \\
$\mathbf{r}$ & Received Signals \\
$\mathbf{b}_{r}$ & Receive Beamformer \\
$y$ & Received Beamformed Signal \\
$y^{\prime}$ & Received Canceled Signal \\
\hline
\end{tabular}

(b)

Figure 2.2: (a) Signal flow diagram of the ALSTAR phased array with multi-channel observation receivers providing access to the distorted and noisy transmitted signals for SIC [1]. (b) Table of symbols and corresponding definitions. 
and $\mathbf{M} \in \mathbb{C}^{K \times J}$ describes the mutual coupling channel between transmitting and receiving elements. The received signals are combined by a receive beamformer,

$$
y(n)=\mathbf{b}_{r}^{\mathrm{H}}\left[\mathbf{s}(n)+\mathbf{M t}(n)+\mathbf{n}_{r}(n)\right],
$$

where $\mathbf{b}_{r} \in \mathbb{C}^{K \times 1}$ is the vector of receive beamforming coefficients and $\mathbf{n}_{r}(n)$ is zeromean complex Gaussian receiver noise with $\mathbf{N}_{r}=E\left[\mathbf{n}_{r} \mathbf{n}_{r}^{\mathrm{H}}\right]=\operatorname{Diag}\left(E\left[\mathbf{r r}^{\mathrm{H}}\right]\right) / \eta_{r}+\sigma_{r}^{2} I$. $\eta_{r}$ is the SNR of each receiver and $\sigma_{r}^{2}$ is the receiver thermal noise power. Note that our model for the receive channels is linear AWGN with two noise components. The first noise component models the limited dynamic range of the channel. This component dominates under strong self-interference. The second term represents the absolute thermal noise figure of the receive channels themselves under low-power excitation. 


\subsubsection{System SINR}

Assuming $x(n), \mathbf{s}(n), \mathbf{n}_{t}(n)$, and $\mathbf{n}_{r}(n)$ are all mutually independent and uncorrelated, we obtain the following receive power terms,

$$
\begin{aligned}
P_{y}^{s} & =\mathbf{b}_{r}^{\mathrm{H}} \mathbf{R}_{s s} \mathbf{b}_{r}, \\
P_{y}^{x} & =\mathbf{b}_{r}^{\mathrm{H}} \mathbf{M} \mathbf{b}_{t} \mathbf{b}_{t}^{\mathrm{H}} \mathbf{M}^{\mathrm{H}} \mathbf{b}_{r}, \\
P_{y}^{n t} & =\mathbf{b}_{r}^{\mathrm{H}} \mathbf{M} \mathbf{N}_{t} \mathbf{M}^{\mathrm{H}} \mathbf{b}_{r}, \\
P_{y}^{n r} & =\mathbf{b}_{r}^{\mathrm{H}} \mathbf{N}_{r} \mathbf{b}_{r},
\end{aligned}
$$

where $P_{y}^{s}$ is the received signal of interest power, $P_{y}^{x}$ is the self-interference power due to the transmitted signal, $P_{y}^{n t}$ is the self-interference power due to the transmitted noise, and $P_{y}^{n r}$ is the receiver noise power in the received beam. Without SIC, the resulting SINR of the received beam would be

$$
\mathrm{SINR}=\frac{P_{y}^{s}}{P_{y}^{x}+P_{y}^{n t}+P_{y}^{n r}}
$$

Note that the presence of self-interference not only introduces the terms $P_{y}^{x}$ and $P_{y}^{n t}$ but may also result in a significant increase in $P_{y}^{n r}$ due to limited receiver dynamic range under high-power excitation. During active transmission, the coupled transmitted signal power $P_{y}^{x}$, coupled transmitted noise power $P_{y}^{n t}$, and receiver noise $P_{y}^{n r}$ 
will generally overwhelm the desired signal power $P_{y}^{s}$ and prevent effective STAR operation. Techniques to mitigate or cancel self-interference and noise are discussed next.

\subsubsection{Mitigation of Transmitted Signal and Noise}

Consider a typical digital SIC scheme, depicted in Fig. 2.3, which removes selfinterference from the received signal $y(n)$ by subtracting a filtered copy of the transmitted signal $x(n)$,

$$
y^{\prime}(n)=y(n)+c x(n) .
$$

If the coupling environment $\mathbf{M}$ has been estimated by an array self-calibration or channel sounding technique (e.g. [77, 178, 131]), then setting $c=-\mathbf{b}_{r}^{\mathrm{H}} \mathbf{M} \mathbf{b}_{t}$ results in

$$
y^{\prime}(n)=\mathbf{b}_{r}^{\mathrm{H}}\left[\mathbf{s}(n)+\mathbf{M n}_{t}(n)+\mathbf{n}_{r}(n)\right] .
$$

Provided that the mutual coupling channel model is sufficiently accurate, this simple method may effectively eliminate the interference term $P_{y}^{x}$. Unfortunately, this approach alone is not generally sufficient to support effective STAR operation in a phased array, as the residual noise terms may still overwhelm the desired signal power, i.e., $P_{y}^{n t}+P_{y}^{n r} \gg P_{y}^{s}$. 


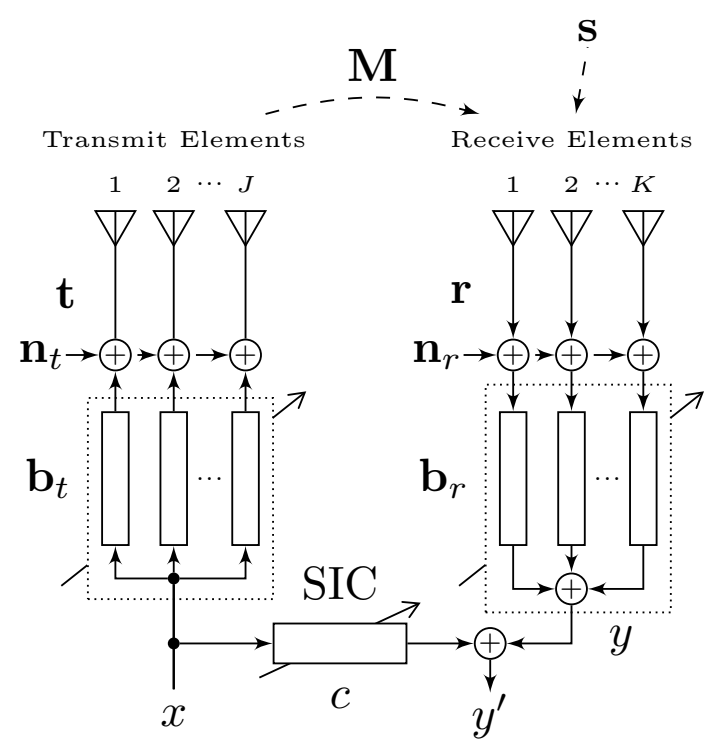

Figure 2.3: Signal flow diagram of a digital phased array with signal-only digital SIC.

Rather than only using knowledge of the signal $x(n)$ for SIC, the actual radiated waveform at each transmitting element $\mathbf{t}(n)$ may be measured as shown in the architecture depicted in Fig. 2.2(a), In this way, not only the transmitted signal but also the transmitted noise and distortion may be canceled. To accomplish this, observation receivers have been included at each transmit channel, whose signals are combined with a multi-channel cancellation filter $\mathbf{b}_{c}$. The observation channels are simply standard receive channels that have been coupled to the transmitter output while the element is transmitting. Because each element in a fully digital array has a dedicated digital receive channel with independent hardware, and these receivers are otherwise unused while the element is transmitting, implementing observation channels in a fully digital array does not significantly impact cost or complexity. An 


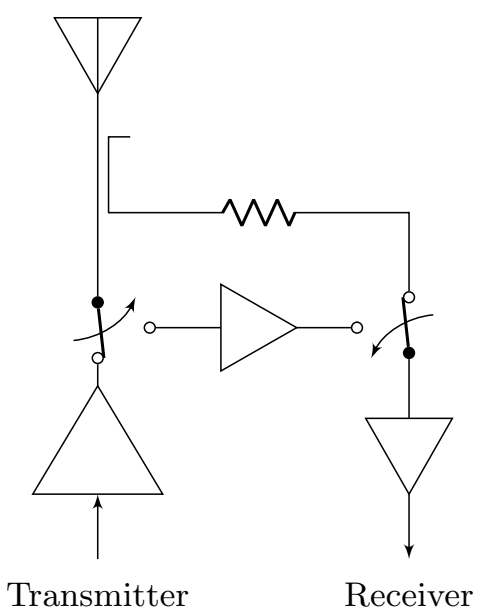

Figure 2.4: Example front end of a $\mathrm{T} / \mathrm{R}$ module that supports ALSTAR signal, noise, and distortion cancellation. In transmit mode (shown) the receiver measures the transmitted signal and noise through an attenuator. In receive mode (arrows) the receiver is connected to the antenna through a low noise amplifier and the transmitter is disabled [1].

example transmit/receive $(\mathrm{T} / \mathrm{R})$ module configuration for realizing the coupled observation path is shown in Fig. 2.4. The observed transmitted signal vector o $(n) \in \mathbb{C}^{J \times 1}$ is

$$
\mathbf{o}(n)=\mathbf{H}_{o}\left[\mathbf{t}+\mathbf{n}_{o}(n)\right],
$$

where $\mathbf{H}_{o} \in \mathbb{C}^{J \times J}$ is a diagonal matrix which represents the fixed attenuator between each transmit channel and its corresponding observation receive channel as shown in Fig. 2.4. Like $\mathbf{M}, \mathbf{H}_{o}$ must be estimated in practice by a channel sounding technique. The attenuators represented by $\mathbf{H}_{o}$ are used to prevent observation channel saturation. The observation channel is AWGN with $\mathbf{n}_{o}(n) \sim \mathcal{C N}\left(\mathbf{0}, \mathbf{N}_{o}\right)$ where $\mathbf{N}_{o}=E\left[\mathbf{n}_{o} \mathbf{n}_{o}^{\mathrm{H}}\right]=$ $\operatorname{Diag}\left(\mathbf{b}_{t} \mathbf{b}_{t}^{\mathrm{H}}\right) / \eta_{r}$. The observation signals $\mathbf{o}(n)$ are combined via multi-channel filter 
$\mathbf{b}_{c}$ and added to $y(n)$ to obtain the isolated received signal

$$
y^{\prime}(n)=y(n)+\mathbf{b}_{c}^{\mathrm{H}} \mathbf{o}(n) .
$$

Setting $\mathbf{b}_{c}^{\mathrm{H}}=-\mathbf{b}_{r}^{\mathrm{H}} \mathbf{M} \mathbf{H}_{o}^{-1}$, the resulting signal is

$$
y^{\prime}(n)=\mathbf{b}_{r}^{\mathrm{H}}\left[\mathbf{s}(n)-\mathbf{M n}_{o}(n)+\mathbf{n}_{r}(n)\right] .
$$

We see that 2.13 is identical to 2.10 except that the transmit noise $\mathbf{n}_{t}$ has been replaced with the observation noise $\mathbf{n}_{o}$, effectively reducing the residual transmitter noise power by a factor of $\eta_{r} / \eta_{t}$. In many cases this represents a substantial improvement, as it is typical for $\eta_{r} \gg \eta_{t}$, especially under high transmit power. Furthermore, although our model assumes linear AWGN channels, this technique will also effectively cancel transmitter nonlinearities and distortion products which in practice may be significantly above the transmitter noise floor, so long as they are also above the observation noise floor and can be measured by the observation receivers. The resulting SINR for $y^{\prime}(n)$ assuming full channel knowledge and corresponding optimization of $\mathbf{b}_{c}$ is

$$
\mathrm{SINR}^{\prime}=\frac{P_{y}^{s}}{P_{y}^{n o}+P_{y}^{n r}},
$$

where

$$
P_{y}^{n o}=\mathbf{b}_{r}^{\mathrm{H}} \mathbf{M} \mathbf{N}_{o} \mathbf{M}^{\mathrm{H}} \mathbf{b}_{r}
$$


is the noise power in the received canceled signal due to performing SIC with observed signals.

\subsubsection{Mitigation of Observation and Receive Noise}

The remaining noise terms $P_{y}^{n o}$ and $P_{y}^{n r}$ may still significantly degrade the sensitivity of the array during transmission. The receive noise correlation matrix can be expanded as

$$
\begin{aligned}
\mathbf{N}_{r}= & \eta_{r}^{-1} \operatorname{Diag}\left(\mathbf{R}_{s s}\right) \\
& +\eta_{r}^{-1} \operatorname{Diag}\left(\mathbf{M} \mathbf{b}_{t} \mathbf{b}_{t}^{\mathrm{H}} \mathbf{M}^{\mathrm{H}}\right) \\
& +\eta_{r}^{-1} \eta_{t}^{-1} \operatorname{Diag}\left[\mathbf{M} \operatorname{Diag}\left(\mathbf{b}_{t} \mathbf{b}_{t}^{\mathrm{H}}\right) \mathbf{M}^{\mathrm{H}}\right] \\
& +\sigma_{r}^{2} I .
\end{aligned}
$$

Under STAR operation, self-interference power may be assumed to dominate the incident power at each receive channel, yielding

$$
\begin{aligned}
\mathbf{N}_{r} \approx & \eta_{r}^{-1} \operatorname{Diag}\left(\mathbf{M} \mathbf{b}_{t} \mathbf{b}_{t}^{\mathrm{H}} \mathbf{M}^{\mathbf{H}}\right) \\
& +\eta_{r}^{-1} \eta_{t}^{-1} \operatorname{Diag}\left[\mathbf{M} \operatorname{Diag}\left(\mathbf{b}_{t} \mathbf{b}_{t}^{\mathrm{H}}\right) \mathbf{M}^{\mathrm{H}}\right] \\
& +\sigma_{r}^{2} I .
\end{aligned}
$$


The total residual noise in the receive beam $P_{n}=P_{y}^{n o}+P_{y}^{n r}$ can then be expressed as a quadratic form of the receive beamformer $\mathbf{b}_{r}$,

$$
P_{n}=\mathbf{b}_{r}^{\mathrm{H}} \mathbf{M}_{b r} \mathbf{b}_{r}
$$

where

$$
\begin{aligned}
\mathbf{M}_{b r}= & \eta_{r}^{-1} \mathbf{M} \operatorname{Diag}\left(\mathbf{b}_{t} \mathbf{b}_{t}^{\mathrm{H}}\right) \mathbf{M}^{\mathrm{H}} \\
& +\eta_{r}^{-1} \operatorname{Diag}\left(\mathbf{M} \mathbf{b}_{t} \mathbf{b}_{t}^{\mathrm{H}} \mathbf{M}^{\mathrm{H}}\right) \\
& +\eta_{r}^{-1} \eta_{t}^{-1} \operatorname{Diag}\left[\mathbf{M} \operatorname{Diag}\left(\mathbf{b}_{t} \mathbf{b}_{t}^{\mathrm{H}}\right) \mathbf{M}^{\mathrm{H}}\right] \\
& +\sigma_{r}^{2} I .
\end{aligned}
$$

Using the fact that $\mathbf{a}^{\mathrm{H}} \operatorname{Diag}\left(\mathbf{b b}^{\mathrm{H}}\right) \mathbf{a}=\mathbf{b}^{\mathrm{H}} \operatorname{Diag}\left(\mathbf{a a}^{\mathrm{H}}\right) \mathbf{b}$ for $\mathbf{a}, \mathbf{b} \in \mathbb{C}^{N, 1}, 2.18$ may also be refactored as a quadratic form of the transmit beamformer $\mathbf{b}_{t}$,

$$
P_{n}=\mathbf{b}_{t}^{\mathrm{H}} \mathbf{M}_{b t} \mathbf{b}_{t}
$$


where

$$
\begin{aligned}
\mathbf{M}_{b t}= & \eta_{r}^{-1} \operatorname{Diag}\left(\mathbf{M}^{\mathrm{H}} \mathbf{b}_{r} \mathbf{b}_{r}^{\mathrm{H}} \mathbf{M}\right) \\
& +\eta_{r}^{-1} \mathbf{M}^{\mathrm{H}} \operatorname{Diag}\left(\mathbf{b}_{r} \mathbf{b}_{r}^{\mathrm{H}}\right) \mathbf{M} \\
& +\eta_{r}^{-1} \eta_{t}^{-1} \operatorname{Diag}\left[\mathbf{M}^{\mathrm{H}} \operatorname{Diag}\left(\mathbf{b}_{r} \mathbf{b}_{r}^{\mathrm{H}}\right) \mathbf{M}\right] \\
& +\frac{\sigma_{r}^{2}}{P_{t}} I
\end{aligned}
$$

In (2.21) we have set $\left\|\mathbf{b}_{t}\right\|^{2}=P_{t}$ and $\left\|\mathbf{b}_{r}\right\|^{2}=1$. Expressions 2.18) and (2.20) highlight the fact that optimizing the transmit and receive beamformers can both reduce residual noise in the receive beam due to self-interference. Indeed, the symmetry of (2.19) and 2.21) suggests that transmit and receive beamforming are equally important and that both ought to be optimized in order to realize high-isolation sufficient for effective STAR operation of a phased array.

Specifically, optimization of the receive beamformer can dramatically reduce the contribution of the observation noise, which is represented by the first line of (2.19). This receive beamforming optimization problem is diagonally loaded by the elevated receive noise floor due to high-power self-interference and limited receiver dynamic range, which is represented in the second and third lines of $(2.19)$, and by the receiver thermal noise power, given in the last line of (2.19).

In turn, optimization of the transmit beamformer provides a method to mitigate 
receiver noise by reducing the incident power at each receive channel, thus improving receiver sensitivity and preventing saturation. This is represented in the second line of 2.21. The transmit beamforming optimization problem is likewise diagonally loaded by the observation noise and the elevated receiver noise floor due to the coupled transmitter noise, represented in the first and third lines of (2.21), and by the receiver

thermal noise power, given in the last line of (2.21). Optimizing the transmit and receive beamformers to minimize noise while forming the desired beam in the far field is discussed in the next section.

\subsection{Effective Isotropic Isolation}

For STAR systems, transmit/receive isolation is often used as a critical metric. Isolation is defined as the ratio of transmitted power $P_{t}$ to residual noise in the receiver $P_{n}$,

$$
I=\frac{P_{t}}{P_{n}}
$$

However, for directional systems, this definition must be expanded to account for the gains achievable via beamforming across the transmitting and receiving antennas. Therefore, we use the EII metric, which is the ratio of Effective Isotropic Radiated 
Power (EIRP) to Effective Isotropic Sensitivity (EIS) [1],

$$
\mathrm{EII}=\frac{\mathrm{EIRP}}{\mathrm{EIS}} .
$$

EIRP - the power required by a theoretical isotropic transmitter to provide equivalent illumination in the desired direction — may be defined for a phased array as

$$
\operatorname{EIRP}\left(\phi, \theta, \mathbf{b}_{t}\right)=g(\phi, \theta) \mathbf{b}_{t}^{\mathrm{H}} \mathbf{q}_{t}(\phi, \theta) \mathbf{q}_{t}^{\mathrm{H}}(\phi, \theta) \mathbf{b}_{t},
$$

where

$$
\mathbf{q}_{t}(\phi, \theta)=e^{-j \frac{2 \pi}{\lambda}\left(\mathbf{x}_{t} \cos (\phi) \sin (\theta)+\mathbf{y}_{t} \sin (\phi) \sin (\theta)\right)}
$$

is the steering vector for a beam $\phi$ rad from the positive $x$-axis in the array plane and $\theta \mathrm{rad}$ from the positive $z$-axis (into the page) as given in Fig. 2.1(a), $\mathbf{x}_{t}$ and $\mathbf{y}_{t}$ are the $x$ and $y$ positions of each transmitting antenna element in the array plane. $g(\phi, \theta)$ is the embedded element gain of the array. EIS - the noise floor of a theoretical isotropic receiver with equivalent sensitivity in the desired direction - may be defined for a phased array as

$$
\mathrm{EIS}=\frac{P_{n}}{G_{r}},
$$

where $P_{n}$ is defined in (2.18) and $(2.20)$ and $G_{r}$ is the received signal gain,

$$
G_{r}\left(\phi, \theta, \mathbf{b}_{r}\right)=g(\phi, \theta) \mathbf{b}_{r}^{\mathrm{H}} \mathbf{q}_{r}(\phi, \theta) \mathbf{q}_{r}^{\mathrm{H}}(\phi, \theta) \mathbf{b}_{r},
$$


where $\mathbf{q}_{r}(\phi, \theta)$ is defined in the same way as its transmit equivalent in 2.25 , except that the sign of the complex exponent in $\mathbf{q}_{r}(\phi, \theta)$ is positive. The difference between the transmit and receive steering vectors accounts for the fact that the receive beamformer is applied with the Hermitian transpose. Assembling terms we arrive at two equivalent expressions for EII in an ALSTAR array

$$
\begin{aligned}
\mathrm{EII} & =G_{r}\left(\phi, \theta, \mathbf{b}_{r}\right) g(\phi, \theta) \frac{\mathbf{b}_{t}^{\mathrm{H}} \mathbf{q}_{t}(\phi, \theta) \mathbf{q}_{t}^{\mathrm{H}}(\phi, \theta) \mathbf{b}_{t}}{\mathbf{b}_{t}^{\mathrm{H}} \mathbf{M}_{b t} \mathbf{b}_{t}}, \\
& =\operatorname{EIRP}\left(\phi, \theta, \mathbf{b}_{t}\right) g(\phi, \theta) \frac{\mathbf{b}_{r}^{\mathrm{H}} \mathbf{q}_{r}(\phi, \theta) \mathbf{q}_{r}^{\mathrm{H}}(\phi, \theta) \mathbf{b}_{r}}{\mathbf{b}_{r}^{\mathrm{H}} \mathbf{M}_{b r} \mathbf{b}_{r}} .
\end{aligned}
$$

Note that (2.28a) is a generalized Rayleigh quotient in $\mathbf{b}_{t}$ (holding $\mathbf{b}_{r}$ constant) and (2.28b) is a generalized Rayleigh quotient in $\mathbf{b}_{r}$ (holding $\mathbf{b}_{t}$ constant). Given estimates of $\mathbf{M}$ and $\mathbf{H}_{o}$, each of these quotients can be maximized individually, allowing us to iterate toward an optimal solution for $\mathbf{b}_{t}$ and $\mathbf{b}_{r}$. Specifically, we alternate between solving the following,

$$
\begin{aligned}
& \mathbf{b}_{t}=\alpha \mathbf{M}_{b t}^{-1} \mathbf{q}_{t}(\phi, \theta), \text { s.t. }\left\|\mathbf{b}_{t}\right\|^{2}=P_{t}, \\
& \mathbf{b}_{r}=\beta \mathbf{M}_{b r}^{-1} \mathbf{q}_{r}(\phi, \theta), \text { s.t. }\left\|\mathbf{b}_{r}\right\|^{2}=1
\end{aligned}
$$

where $\alpha$ and $\beta$ are arbitrary scale factors chosen to satisfy the magnitude constraints [132. Note that $\mathbf{M}_{b t}$ and $\mathbf{M}_{b r}$ are recalculated at each iteration, and the iterations continue until $\mathbf{b}_{t}$ and $\mathbf{b}_{r}$ converge. This approach is guaranteed to converge because each evaluation of $(2.29)$ and 2.30 is non-decreasing in EII, which is bounded above 
by

$$
\mathrm{EII}_{\text {max }}=\frac{P_{t} J K g^{2}(\phi, \theta)}{\sigma_{r}^{2}}
$$

Alternating optimization over the transmit and receive beamformers does not necessarily find a unique globally optimal solution because the objective function (i.e., EII) is non-convex in $\mathbf{b}_{t}$ and $\mathbf{b}_{r}$. The non-convex nature of the beamformer optimization problem means that the initialization of the algorithm may cause it to converge to different local optima. Therefore, in order to increase the likelihood of finding a solution nearer the globally optimal solution and in order to determine the difference in performance achieved at the various local optima, the iterative procedure described above was initialized with the non-adaptive transmit and receive beamformers

$$
\begin{aligned}
& \mathbf{b}_{t, n a}=\sqrt{P_{t} / J} \cdot \mathbf{q}_{t}(\phi, \theta), \\
& \mathbf{b}_{r, n a}=\sqrt{1 / K} \cdot \mathbf{q}_{r}(\phi, \theta),
\end{aligned}
$$

as well as 100 randomly generated transmit and receive beamformers scaled to meet the transmit power and receive gain constraints in 2.29 and 2.30 . Note that the non-adaptive transmit and receive beamformers - 2.32 and 2.33 - maximize EIRP and receive array gain. We will show empirically that alternating optimization is generally effective at obtaining near-optimal solutions.

In Section 2.4, we compare the performance of the ALSTAR array in three different configurations in order to clarify the relative contributions of SIC and adaptive 
beamforming. We consider the non-adaptive beamformers without SIC, the nonadaptive beamformers with SIC, and the adaptive beamformers with SIC. The difference between the first and second configurations demonstrates the contribution to EII made by SIC alone. The difference between the second and third configurations demonstrates the gain in EII made possible by adaptive beamforming. If the configuration uses the non-adaptive beamformers, no optimization is performed and the non-adaptive beamformers - 2.32 and 2.33 - are substituted into the expression for EII. Without SIC, the interference and noise covariance matrices $\mathbf{M}_{b r}$ and $\mathbf{M}_{b t}$ become

$$
\begin{aligned}
\widetilde{\mathbf{M}}_{b r}= & \mathbf{M b}_{t} \mathbf{b}_{t}^{\mathrm{H}} \mathbf{M}^{\mathrm{H}}+\eta_{t}^{-1} \mathbf{M} \operatorname{Diag}\left(\mathbf{b}_{t} \mathbf{b}_{t}^{\mathrm{H}}\right) \mathbf{M}^{\mathrm{H}} \\
& +\eta_{r}^{-1} \operatorname{Diag}\left(\mathbf{M} \mathbf{b}_{t} \mathbf{b}_{t}^{\mathrm{H}} \mathbf{M}^{\mathrm{H}}\right) \\
& +\eta_{r}^{-1} \eta_{t}^{-1} \operatorname{Diag}\left[\mathbf{M} \operatorname{Diag}\left(\mathbf{b}_{t} \mathbf{b}_{t}^{\mathrm{H}}\right) \mathbf{M}^{\mathrm{H}}\right] \\
& +\sigma_{r}^{2} I . \\
\widetilde{\mathbf{M}}_{b t}= & \mathbf{M}^{\mathrm{H}} \mathbf{b}_{r} \mathbf{b}_{r}^{\mathrm{H}} \mathbf{M}+\eta_{t}^{-1} \operatorname{Diag}\left(\mathbf{M}^{\mathrm{H}} \mathbf{b}_{r} \mathbf{b}_{r}^{\mathrm{H}} \mathbf{M}\right) \\
& +\eta_{r}^{-1} \mathbf{M}^{\mathrm{H}} \operatorname{Diag}\left(\mathbf{b}_{r} \mathbf{b}_{r}^{\mathrm{H}}\right) \mathbf{M} \\
& +\eta_{r}^{-1} \eta_{t}^{-1} \operatorname{Diag}\left[\mathbf{M}^{\mathrm{H}} \operatorname{Diag}\left(\mathbf{b}_{r} \mathbf{b}_{r}^{\mathrm{H}}\right) \mathbf{M}\right] \\
& +\frac{\sigma_{r}^{2}}{P_{t}} I,
\end{aligned}
$$

The first two terms account for the transmitted signal and noise that is coupled through the mutual coupling channel. Also note that the first term in (2.19) and (2.21) 
does not appear in 2.34) and 2.35) because that term describes the observation noise effectively coupled through the mutual coupling channel due to performing SIC with observation channel signals.

\subsubsection{Properties of the Mutual Coupling Channel}

Before presenting the simulation results, we pause to discuss the nature of the mutual coupling channel in an ALSTAR array. Given the prominent presence of $\mathbf{M}$ in 2.19 and 2.21 , the nature of the coupling channel strongly affects the achievable EII. If we assume that $\mathbf{M}$ is full-rank, then $\min (K, J)$ DOF would be required to create perfect nulls at all of the receive antennas. Given that the transmit beamformer has $J-1$ DOF, forming perfect transmit nulls at each receiver requires more transmitters than receivers (i.e., $J>K$ ). As seen in Section 2.2.3 and earlier in Section 2.3, the receive beamformer can also reject the observation noise via null placement. Perfect nulls to reject the observation noise would require more receivers than transmitters (i.e., $K>$ $J)$. These conditions cannot be simultaneously satisfied, suggesting that simultaneous perfect mutual null placement is not possible. Because 2.31) is maximized when $J=K$ for a fixed number of antennas, and because the first and second terms of (2.19) and (2.21) have similar contributions to $P_{n}$, choosing an equal number of transmitters and receivers is a prudent strategy. The strongest possible mutual null placement with $J=K$ would consume every available DOF, leaving none available 
to form the transmit and receive beams for the signal of interest.

However, several practical considerations mitigate this concern. First, perfect mutual coupling information is unavailable in practice, so perfect nulls become impossible even when the necessary DOF are available. Second, perfect nulls are unnecessary in this scenario [76]. The receive beamformer needs only to suppress the observation noise beneath the receive channel noise due to limited dynamic range and receive channel thermal noise. Likewise, the transmit beamformer needs only to suppress the limited dynamic range receive noise beneath the observation noise and receive channel thermal noise. This can be seen in 2.19) and 2.21), where the non-diagonal component represents the noise to be nulled and the diagonal components act as regularization terms that reduce the distortion in the desired main transmit and receive beams. Finally, antennas are often laid out in very regular patterns in phased arrays, which implies strong similarities between the coupling channels across antennas. This often leads to a coupling matrix with a few dominant modes and a high condition number, reducing the number of DOF required to achieve strong mutual nulls. For example, in the case of a linear ALSTAR array that has a contiguous transmit subarray and a contiguous receive subarray, a single end-fire null aimed at the other subarray in the transmit and receive beam would be all that is required to minimize the noise. Fig. 2.5 shows the singular value spectra of the simulated coupling matrices for the aperture partitions shown in Fig. 2.1(a) and Fig. 2.1(b). It appears that the mutual coupling channel for Partition A has approximately 10 dominant directional 


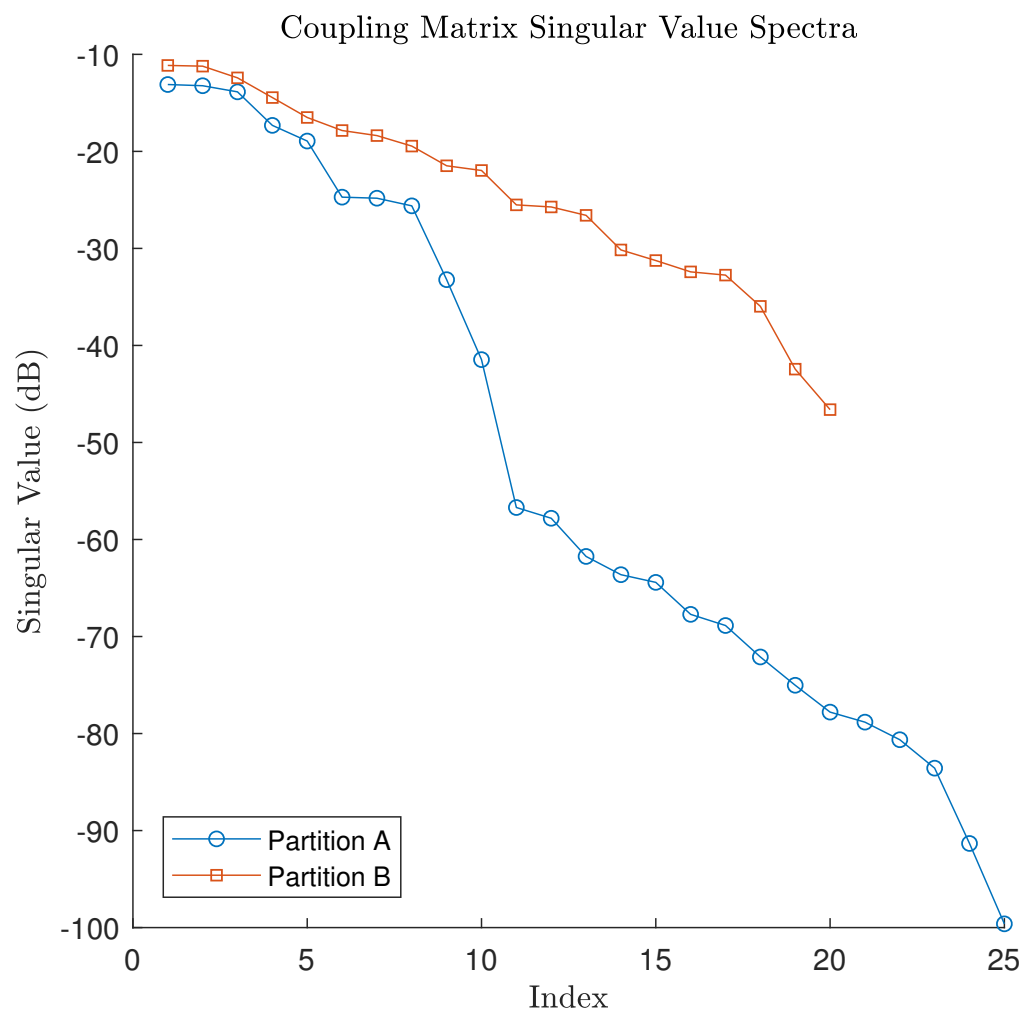

Figure 2.5: The singular value spectra of the coupling matrix $\mathbf{M}$ simulated in Ansys HFSS for the configurations shown in Fig. 2.1(a) and Fig. 2.1(b), respectively.

modes while the singular value spectrum of Partition B is greater than the spectrum of Partition A and has no clear break point between dominant and weak modes. For these reasons, we expect that Partition A will be able to achieve higher EII because the adaptive beamformers will have to use fewer adaptive DOF for noise reduction, increasing the number of DOF that can be dedicated to transmit or receive gain.

The coupling model used in the simulations in this work does not explicitly address a multipath coupling environment. However, the methods proposed in this work do not preclude the consideration of multipath coupling environments. If the modes in $\mathbf{M}$ due 
to multipath do not dominate the direct coupling modes (e.g., in the case of far-field multipath) and thus do not significantly increase the receiver noise, then there will be little effect on the optimal beamformers. Yet, in strong multipath environments, the effective rank of $\mathbf{M}$ will be increased, more DOF will be required for nulls in the transmit and receive beams, resulting in reduced transmit gain, receive gain, and EII.

\subsection{Simulation Results}

A $5 \times 10$ element ALSTAR array of U-slot patch antennas (similar to those described in [133]) was modeled in Ansys HFSS, as discussed in [1] with an element spacing of $\lambda / 2=62 \mathrm{~mm}$ when operated at $2.42 \mathrm{GHz}$. The embedded element gain was modeled as $g(\phi, \theta)=\pi \cos (\theta)$. The array beams were scanned up to $60^{\circ}$ from array normal. The transmit channel dynamic range was $\eta_{t}=45 \mathrm{~dB}$ and the receive channel dynamic range was $\eta_{r}=70 \mathrm{~dB}$. The receive channels had thermal noise power $\sigma_{r}^{2}=-91 \mathrm{dBm}$, which simulates the thermal noise for a $100 \mathrm{MHz}$ bandwidth channel with a $3 \mathrm{~dB}$ noise figure. We present results for $P_{t}=2.5 \mathrm{~W}, 25 \mathrm{~W}, 250 \mathrm{~W}$, and $2500 \mathrm{~W}$, and for both partitions in Fig. 2.1. 


\subsubsection{Partition A}

The array was partitioned as shown in Fig. 2.1(a) into 25 transmit elements (black) and 25 receive elements (gray). Fig. 2.6 shows the EII achieved across scan angles for non-adaptive beamformers without SIC, non-adaptive beamformers with SIC, and adaptive beamformers with SIC at each power level. For the adaptive beamformer results, the median EII across all initializations is plotted with error bars representing the maximum and minimum EII achieved at each scan angle. Non-adaptive beamformer results are only shown for $P_{t}=2500 \mathrm{~W}$ as increasing the transmitted power does not significantly change the EII of the non-adaptive beamformers (with or without SIC) because EIRP scales linearly with $P_{t}$ as do all the terms in 2.19 except the thermal noise floor. For $P_{t}=2500 \mathrm{~W}$ at broadside with adaptive transmit and receive beamformers, a maximum EII of $187.1 \mathrm{~dB}$ is found, an improvement of $108.6 \mathrm{~dB}$ over a non-adaptive array without SIC, and an isolation improvement of $40 \mathrm{~dB}$ over that realizable by SIC alone. As shown in Fig. 2.7, the median $P_{n}$ seen at broadside for the adaptive beamformers with SIC was only $2.2 \mathrm{~dB}$ above the thermal noise floor, an improvement of $43.6 \mathrm{~dB}$ over a non-adaptive array with SIC. Given the demonstrated improvement in EII and $P_{n}$, next we consider the sacrifices made by the adaptive beamformers in EIRP and $G_{r}$. Fig. 2.8 shows the non-adaptive and adaptive EIRP and $G_{r}$ for $P_{t}=2500 \mathrm{~W}$. As before, the median adaptive EIRP and $G_{r}$ are plotted 
with error bars representing the minimum and maximum achieved across all initializations. Within $30^{\circ}$ of broadside, the median reduction in EIRP is $2 \mathrm{~dB}$ and the median $G_{r}$ loss is $1.7 \mathrm{~dB}$ across all initializations. At larger scan angles (out to $60^{\circ}$ ), the median EIRP and $G_{r}$ were both reduced by up to $8.5 \mathrm{~dB}$, when compared with the EIRP and $G_{r}$ of the non-adaptive transmit and receive beamformers, respectively.

Fig. 2.9 and 2.10 show the EII realized across the scan space for $2.5 \mathrm{~W}$ and 2500 $\mathrm{W}$ of transmit power, respectively. These plots show that extremely high isolation is possible across the majority of the scan space, albeit at some modest reduction in aperture efficiency. Note that the horizontal scan space coefficient $k x=\cos (\phi) \cdot \sin (\theta)$, and the vertical scan space coefficient $k y=\sin (\phi) \cdot \sin (\theta)$. Fig. 2.11 shows the transmit power at each transmit element and the interference and noise power at each receive element when the beam is directed toward broadside for 2.11(a) the nonadaptive transmit beamformer and 2.11(b) the adaptive transmit beamformer. This plot demonstrates the effectiveness of the adaptive transmit beamformer at reducing the incident power - and thus reducing the noise power - at the receive elements, keeping them from becoming saturated or being destroyed at high transmit power levels. 


\subsubsection{Partition B}

We also considered the aperture partition shown in Fig. 2.1(b), Fig. 2.12 shows the EII achieved for Partition B. At broadside for $P_{t}=2500 \mathrm{~W}$, with adaptive beamformers and SIC, the ALSTAR array produced $182 \mathrm{~dB}$ of EII at broadside, a gain of $34.4 \mathrm{~dB}$ over the non-adaptive beamformers with $\mathrm{SIC}$ and $110.2 \mathrm{~dB}$ over the non-adaptive beamformers without SIC. Fig. 2.13 shows the noise power at the receiver for the adaptive and non-adaptive beamformers with SIC across the azimuth scan range. Together, the adaptive beamformers and SIC were able to drive the noise power within $5 \mathrm{~dB}$ of the thermal noise power in the median case across azimuth scan angles, power levels, and initializations. At broadside for $P_{t}=2500 \mathrm{~W}$, the adaptive beamformers decreased the received noise by $40.1 \mathrm{~dB}$. Fig. 2.14 compares the EIRP and receive gain of the adaptive and non-adaptive beamformers across the azimuth scan range for Partition $\mathrm{B}$ operated at $P_{t}=2500 \mathrm{~W}$. The adaptive beamformers incurred a median EIRP loss of $3.4 \mathrm{~dB}$ and a median receive gain loss of $5.5 \mathrm{~dB}$ within $30^{\circ}$ of broadside. Considering the full scan space, the median EIRP loss increased to $11.1 \mathrm{~dB}$ and the median receive gain loss increased to $9.2 \mathrm{~dB}$. Fig. 2.15 shows the transmitted power at each transmitting element and the interference and noise power at each receive element. Note that the reduction in interference and noise power at the receivers between Fig. 2.15(a) and Fig. 2.15(b) is less than the reduction seen between Fig. 2.11(a) and Fig. 2.11(b) due to the reduced number of transmit DOF 
and the increased magnitude of the coupling matrix singular values for Partition B as shown in Fig. 2.5 .

\subsection{Beamformer Regularization}

In some applications, array designers and operators may be willing to accept reduced EII to maintain higher EIRP and $G_{r}$. In order to make this possible, we present a regularization technique for the proposed optimization method given in 2.29 and 2.30). If diagonal loading with weight $\gamma \in[0, \infty)$ is applied to the noise covariance matrices in the proposed optimization scheme, we obtain

$$
\begin{aligned}
& \mathbf{b}_{t}=\alpha\left(\mathbf{M}_{b t}+\frac{\gamma}{P_{t}} I\right)^{-1} \mathbf{q}_{t}(\phi, \theta), \text { s.t. }\left\|\mathbf{b}_{t}\right\|^{2}=P_{t}, \\
& \mathbf{b}_{r}=\beta\left(\mathbf{M}_{b r}+\gamma I\right)^{-1} \mathbf{q}_{r}(\phi, \theta), \text { s.t. }\left\|\mathbf{b}_{r}\right\|^{2}=1 .
\end{aligned}
$$

For small values of the regularization coefficient $(\gamma \rightarrow 0)$, the optimization converges to the results given in Section 2.4. As $\gamma \rightarrow \infty$, the EIRP and $G_{r}$ of the final solution will increase toward those of the non-adaptive beamformers, with some loss in EII. In order to explore the trade-off between EIRP or $G_{r}$ and EII, we plot the total array gain $G_{t} \cdot G_{r}$, where $G_{t}=\mathrm{EIRP} / P_{t}$. The total array gain accounts for the fact that some solutions to the optimization described in (2.36) and (2.37) trade $G_{t}$ for $G_{r}$ or vice versa. These plots correspond to the regularized beamformers that obtained 
the maximum EII across initializations for each value of $\gamma$. Fig. 2.16(a) shows the relationship between total array gain and EII at $\theta=30^{\circ}$ for the ALSTAR array configuration of Fig. 2.1(a), as $\gamma$ is varied from $-200 \mathrm{dBm}$ to $30 \mathrm{dBm}$. Fig. 2.16(b) plots the total array gain against the effective noise figure (ENF) of the array, defined as the ratio of the noise power to the thermal noise floor, i.e.,

$$
\mathrm{ENF}=\frac{P_{n}}{\sigma_{r}^{2}}
$$

These results demonstrate that high array gain is achievable with little decrease in EII or increase in ENF, especially at higher power levels.

\subsection{Conclusion}

In this paper we studied the ALSTAR architecture for a fully-digital phased array with digital SIC that can achieve STAR. We explored the EII performance metric that expands the concept of transmit/receive isolation to account for the directional gain of the transmit and receive apertures and the noise injected into the receive channel by digital SIC with measured transmitted signals. This work applied the definition of EII to a signal model that accounted for the limited dynamic range of the transmit and receive $\mathrm{RF}$ front ends. We proposed a method of optimizing the transmit and receive beamformers to maximize the EII between the transmit and 
receive channels and presented results for an example $5 \times 10$ ALSTAR array. The proposed optimization technique achieved high EII over a large scan region while retaining sufficient transmit/receive gain and keeping the receive channel noise near the thermal noise floor. This work demonstrates that adaptive digital beamforming techniques coupled with digital SIC techniques are capable of creating very high isolation between transmit and receive apertures in a digital phased array without the use of electromagnetic isolation techniques or analog SIC hardware. This work also presented a regularization method for the proposed optimization technique that can be used to trade EII for EIRP and $G_{r}$. In the future, we intend to develop application specific performance metrics based on this architecture, and develop more practical and efficient methods for obtaining EII-optimal beamformers. 


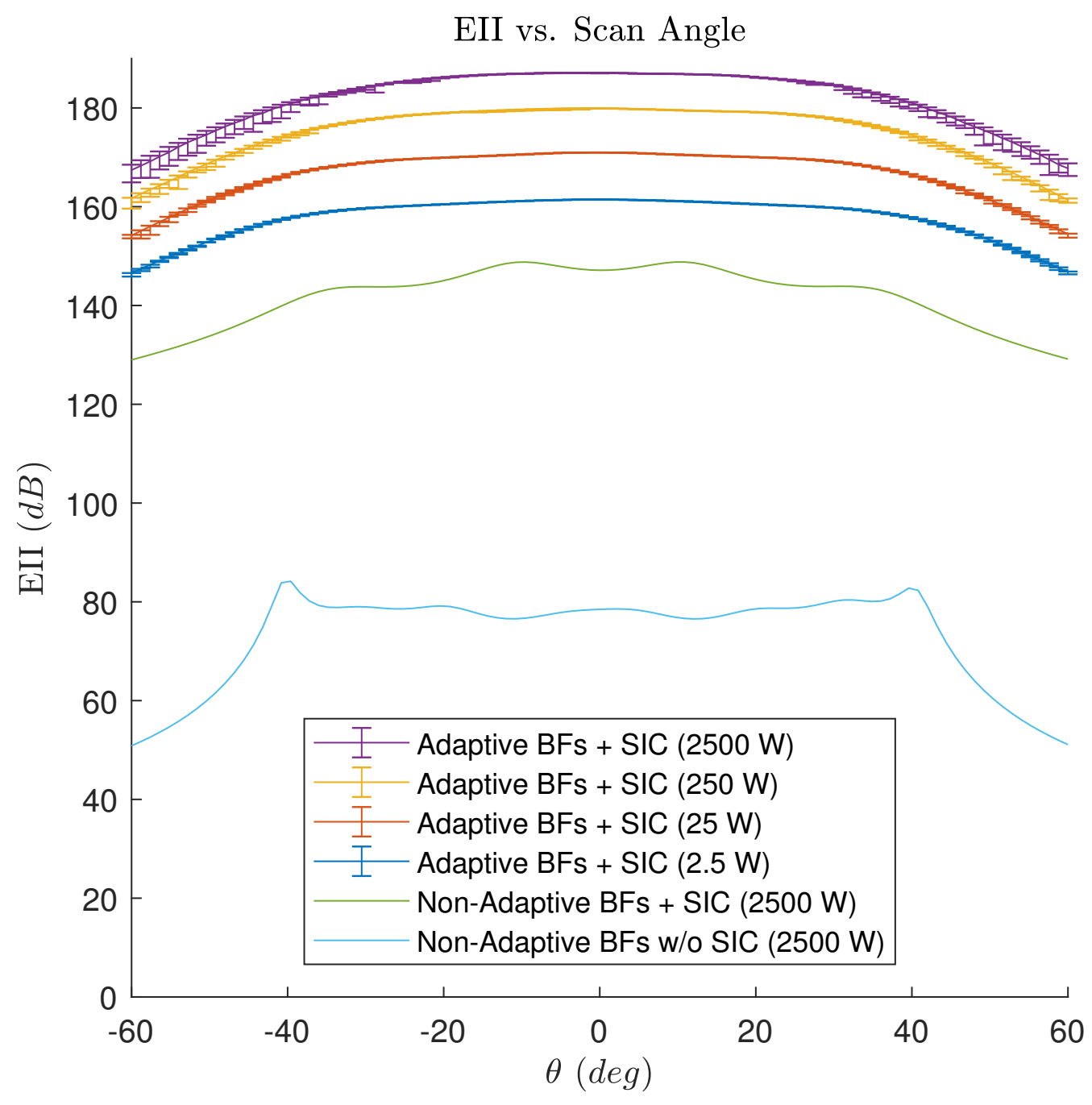

Figure 2.6: Effective Isotropic Isolation for the ALSTAR array configuration of Fig. 2.1(a), plotted across azimuth beam angle for non-adaptive beamformers without SIC at $2500 \mathrm{~W}$, non-adaptive beamformers with SIC at $2500 \mathrm{~W}$, and adaptive beamformers with SIC at $2.5 \mathrm{~W}, 25 \mathrm{~W}, 250 \mathrm{~W}$, and $2500 \mathrm{~W}$ of total transmit power. The median EII for the adaptive beamformers with SIC was calculated across 100 initializations with randomly generated beamformers and one initialization with the non-adaptive beamformers. The error bars indicate the minimum and maximum EII achieved across all initializations. 


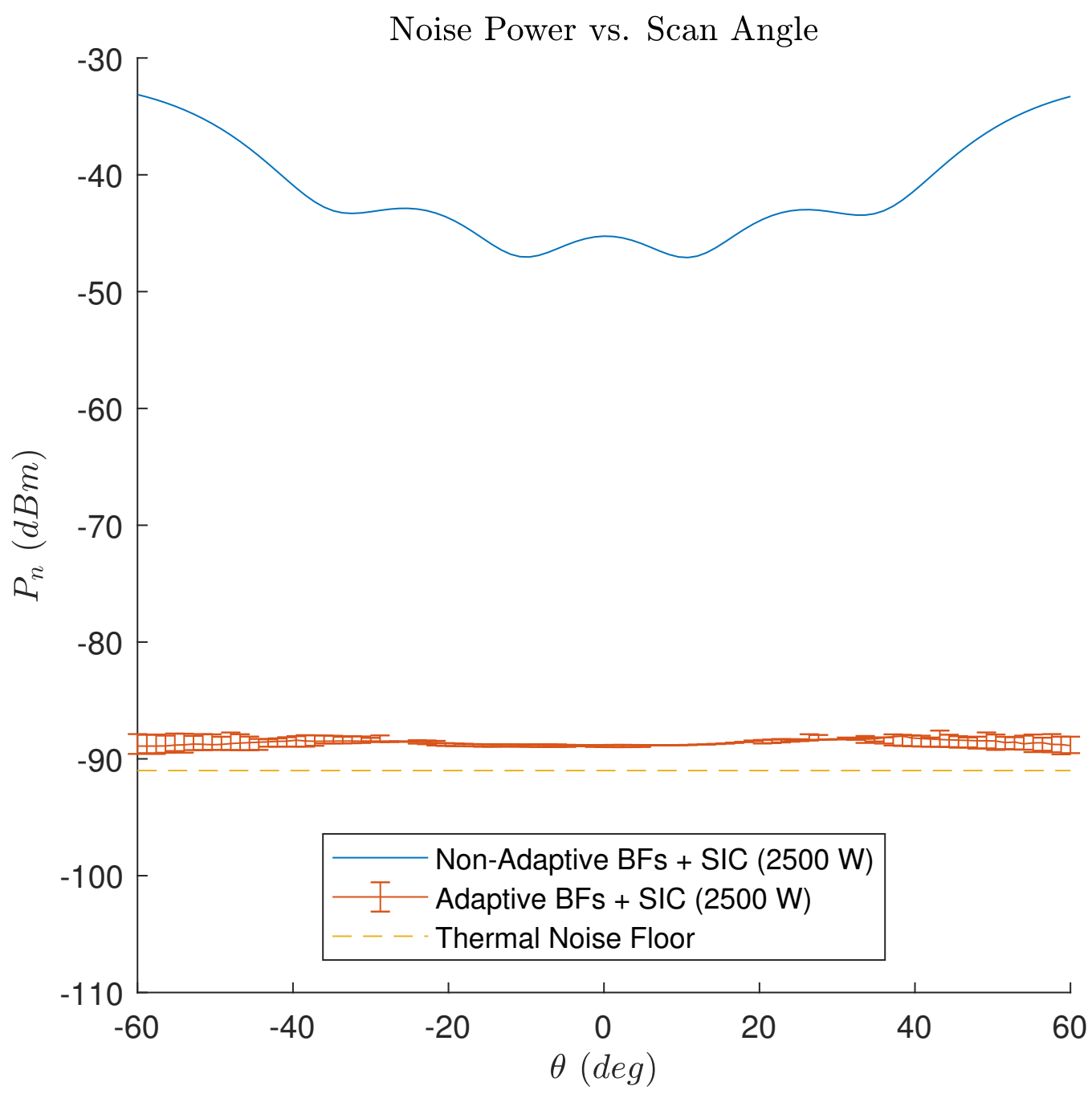

Figure 2.7: Noise power $P_{n}$ in the received signal for the ALSTAR array configuration of Fig.2.1(a), plotted across azimuthal scan angle. The median $P_{n}$ for the adaptive beamformers with SIC is plotted with error bars to indicate the minimum and maximum $P_{n}$ achieved across all initializations. 


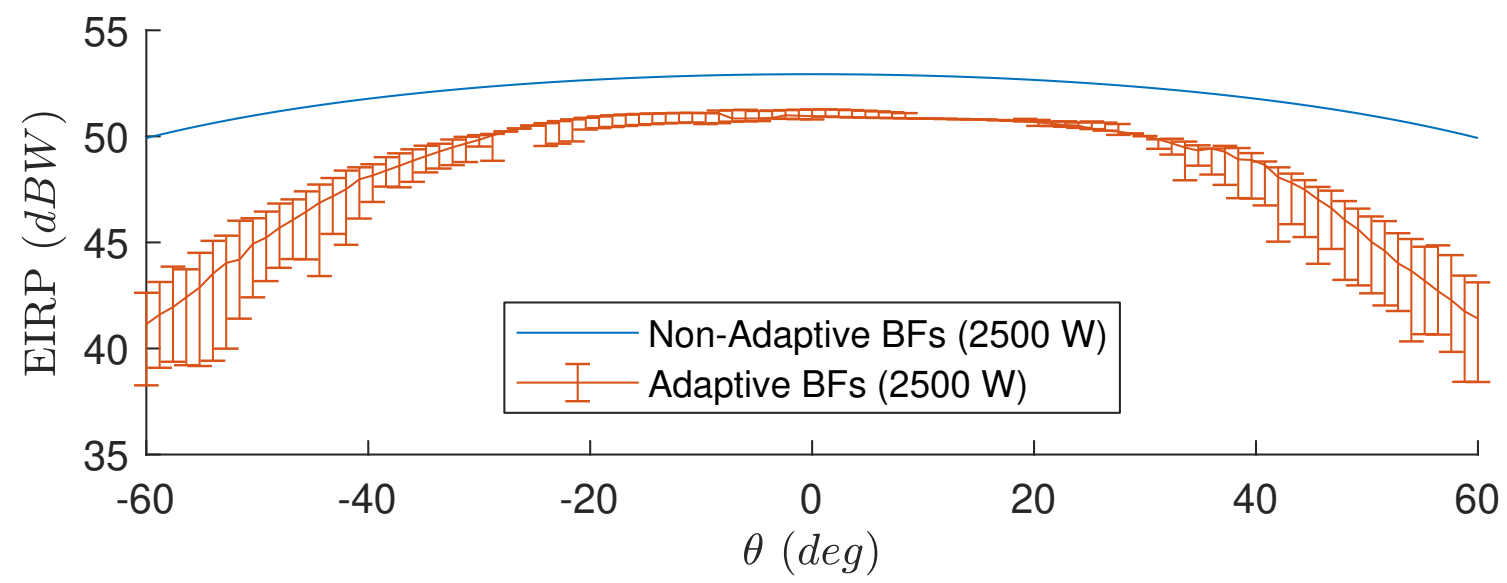

(a) EIRP vs. Azimuth Scan Angle

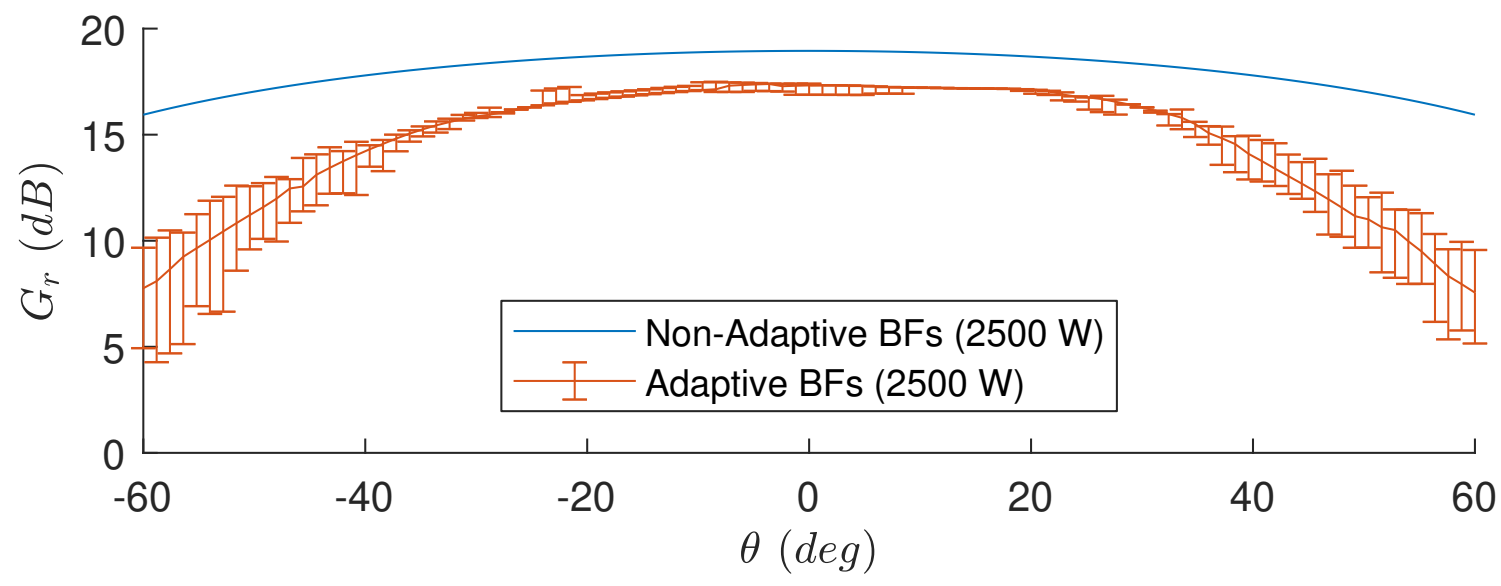

(b) $G_{r}$ vs. Azimuth Scan Angle

Figure 2.8: (a) Effective Isotropic Radiated Power (EIRP) and (b) Receive Gain $\left(G_{r}\right)$ plotted against azimuth beam angle for the non-adaptive and adaptive beamformers for the ALSTAR array configuration of Fig. 2.1(a), The adaptive results show the median EIRP and $G_{r}$ with error bars indicating the minimum and maximum values across initializations. 


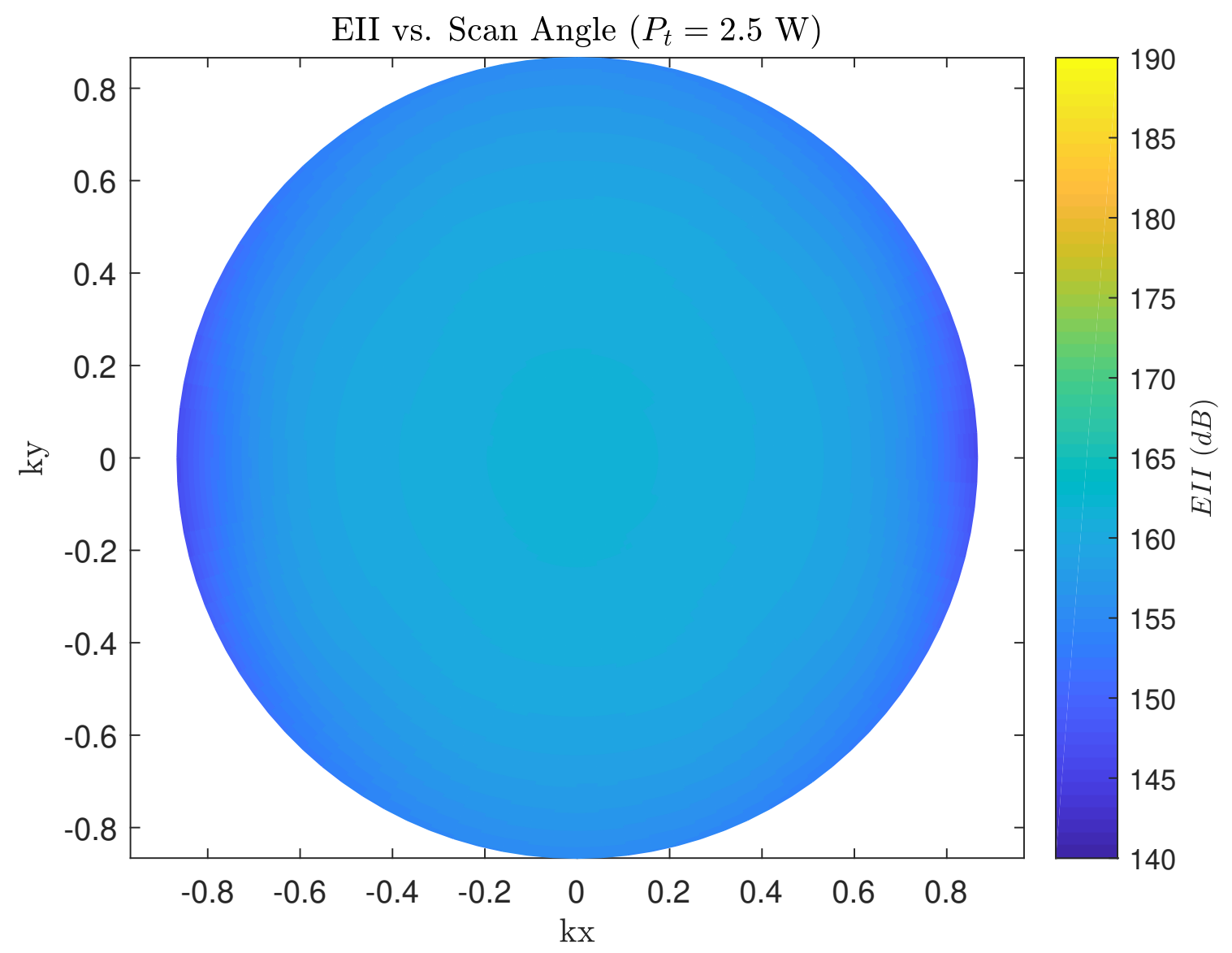

Figure 2.9: EII for the ALSTAR array configuration of Fig. 2.1(a), plotted across the scan space out to $60^{\circ}$ off broadside for the adaptive beamformers at $2.5 \mathrm{~W}$ transmit power. The initial condition for beamformer optimization was $\mathbf{b}_{t}=\mathbf{b}_{t, n a}$ and $\mathbf{b}_{r}=\mathbf{b}_{r, n a}$. 


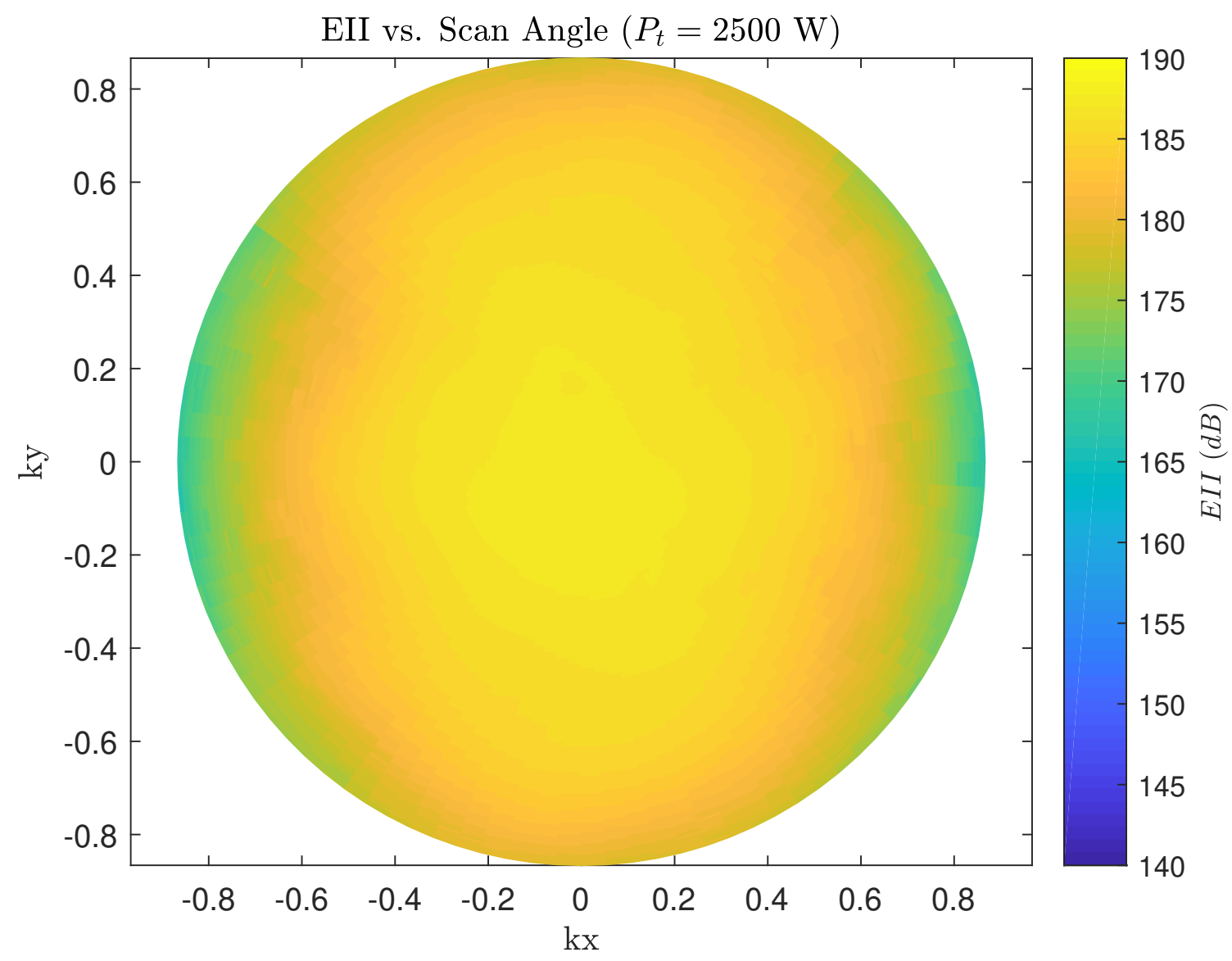

Figure 2.10: EII for the ALSTAR array configuration of Fig. 2.1(a), plotted across the scan space out to $60^{\circ}$ off broadside for the adaptive beamformers at $2500 \mathrm{~W}$ transmit power. The initial condition for beamformer optimization was $\mathbf{b}_{t}=\mathbf{b}_{t, n a}$ and $\mathbf{b}_{r}=\mathbf{b}_{r, n a}$. 


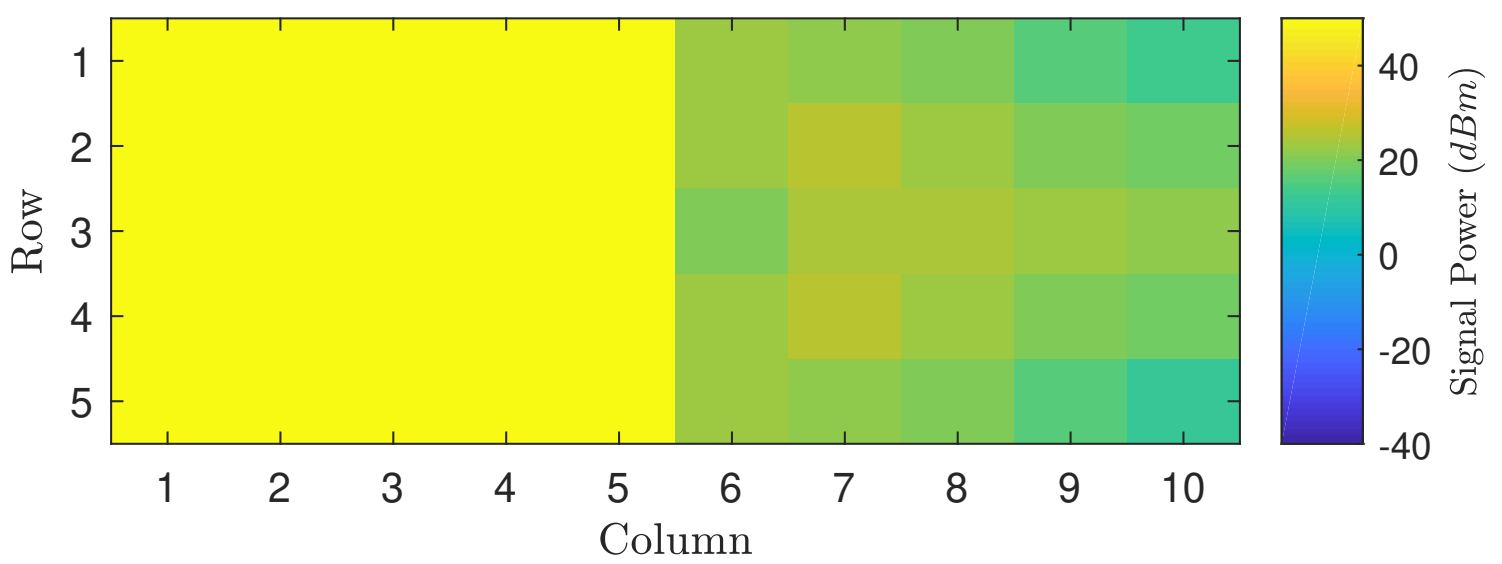

(a) Tx/Rx Element Power - Non-Adaptive Tx Beamformer

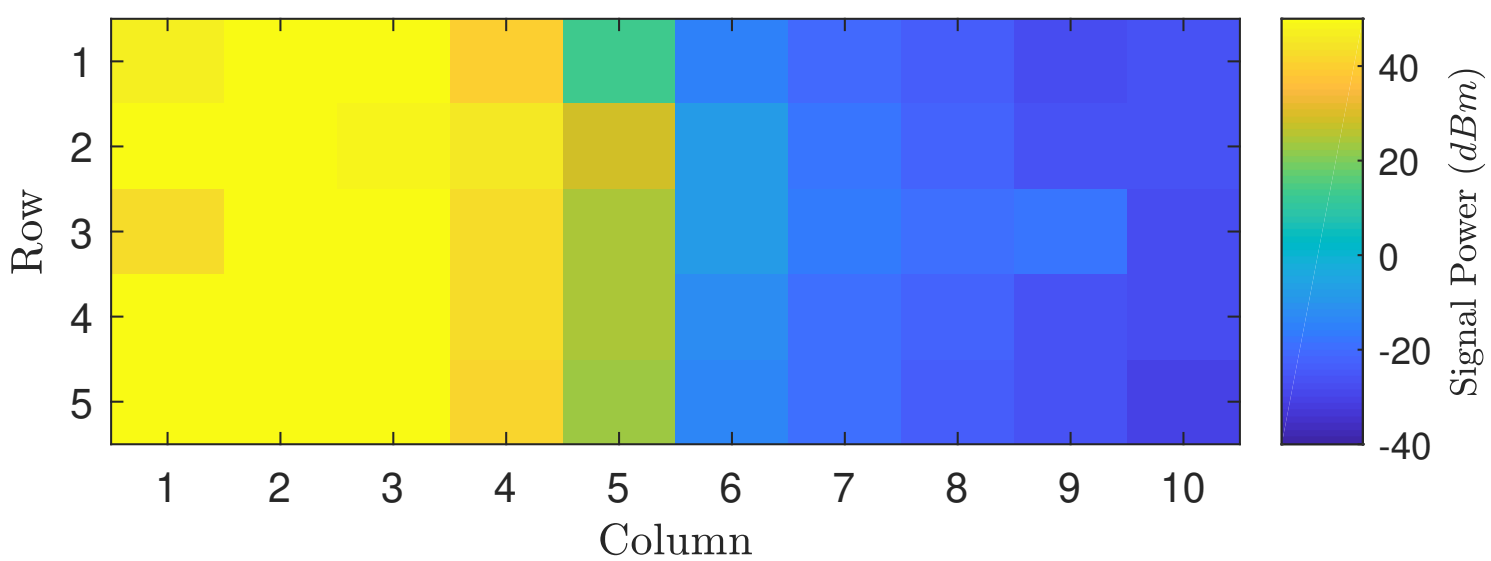

(b) Tx/Rx Element Power - Adaptive Tx Beamformer

Figure 2.11: Comparison of the transmitted power at each element and the incident power at each receive element between (a) the non-adaptive transmit beamformer and (b) the adaptive transmit beamformer for the aperture partition shown in Fig. 2.1(b) with $P_{t}=2500$ W. Significant reduction in receive element incident power was achieved via the adaptive transmit beamformer. 


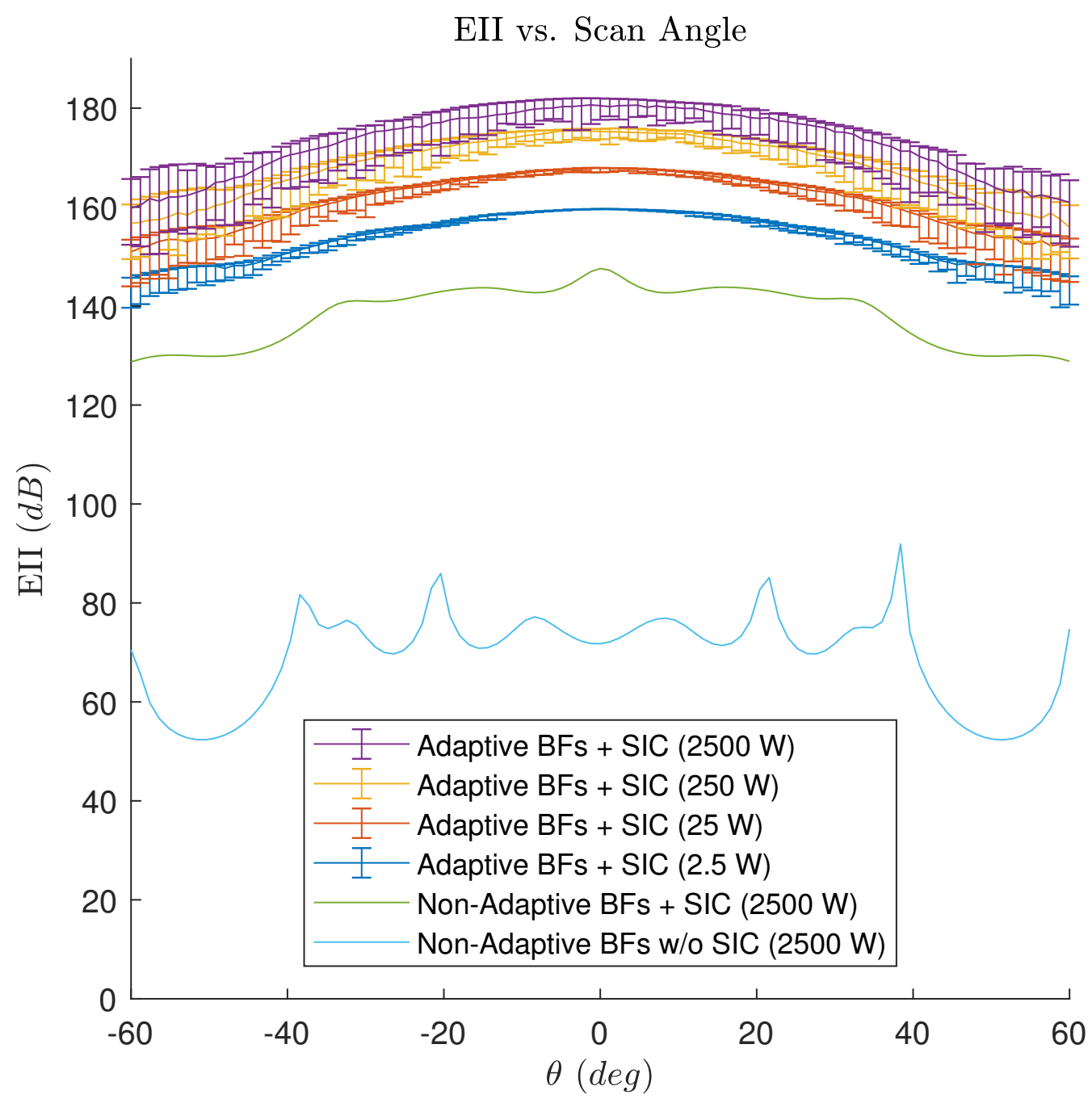

Figure 2.12: Effective Isotropic Isolation for the ALSTAR array configuration of Fig. 2.1(b), plotted across azimuth beam angle for non-adaptive beamformers without SIC at $2500 \mathrm{~W}$, non-adaptive beamformers with SIC at $2500 \mathrm{~W}$, and adaptive beamformers with SIC at $2.5 \mathrm{~W}, 25 \mathrm{~W}, 250 \mathrm{~W}$, and $2500 \mathrm{~W}$ of total transmit power. The median EII for the adaptive beamformers with SIC was calculated over 100 initializations with randomly generated beamformers and one initialization with the non-adaptive beamformers. The error bars indicate the minimum and maximum EII achieved across all initializations. 


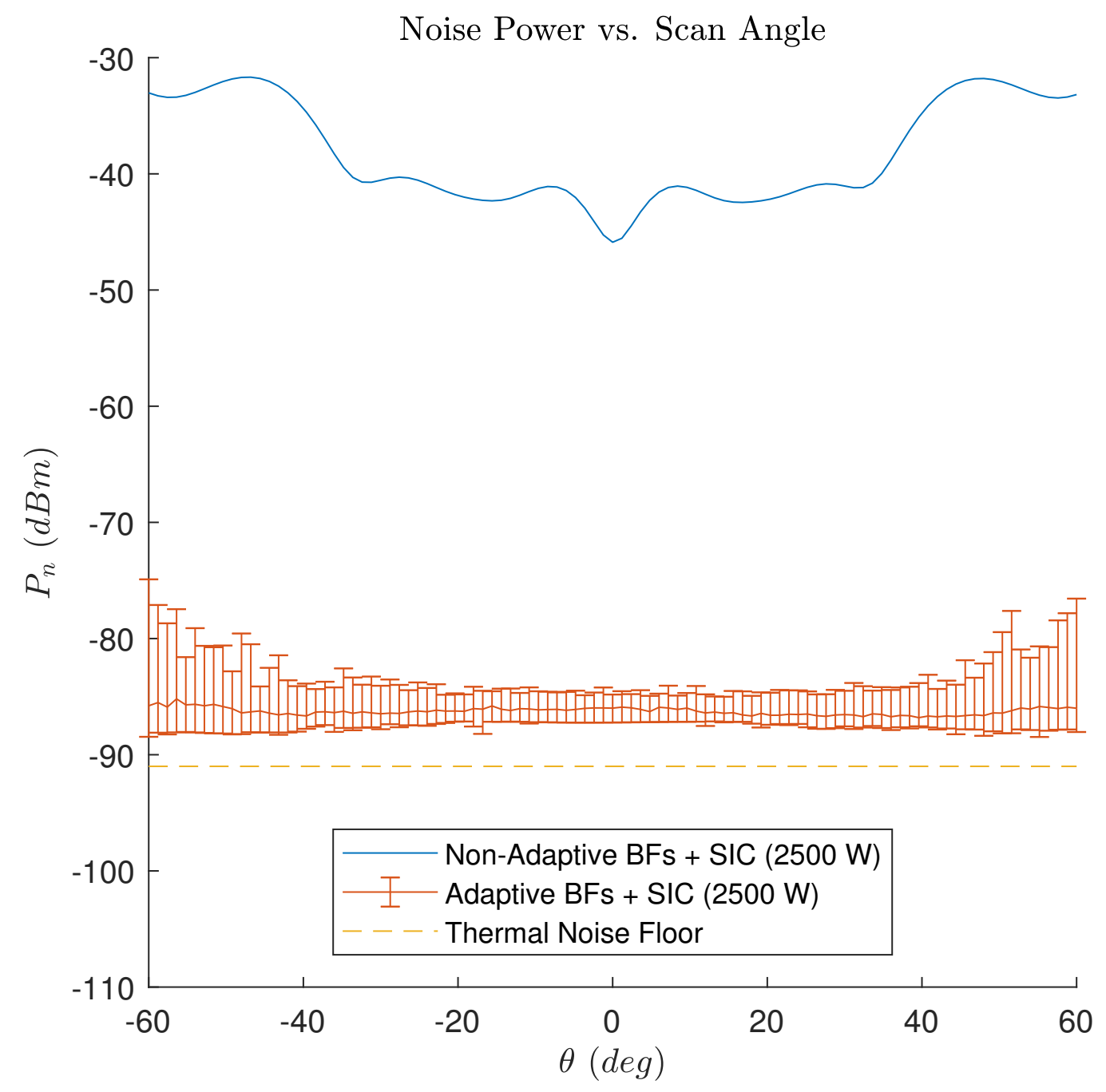

Figure 2.13: Noise power $P_{n}$ in the received signal for the ALSTAR array configuration of Fig. 2.1(b), plotted across azimuthal scan angle. The median $P_{n}$ for the adaptive beamformers with SIC is plotted with error bars to indicate the minimum and maximum $P_{n}$ achieved across all initializations. 


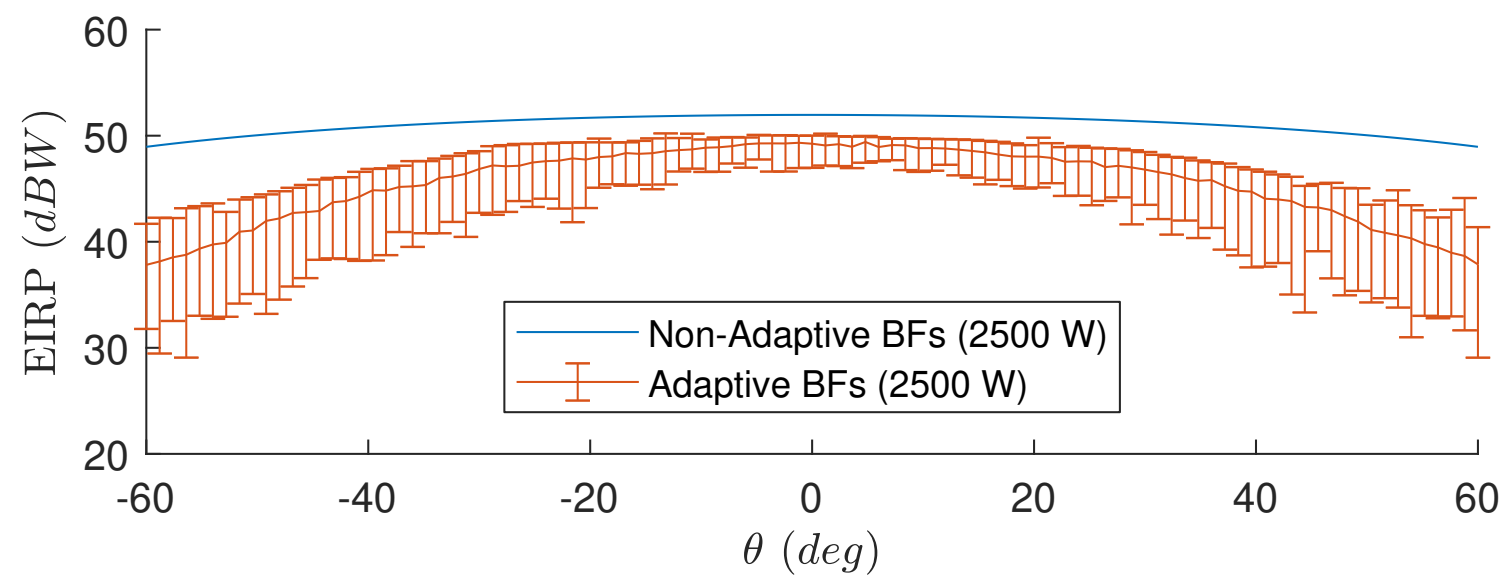

(a) EIRP vs. Azimuth Scan Angle

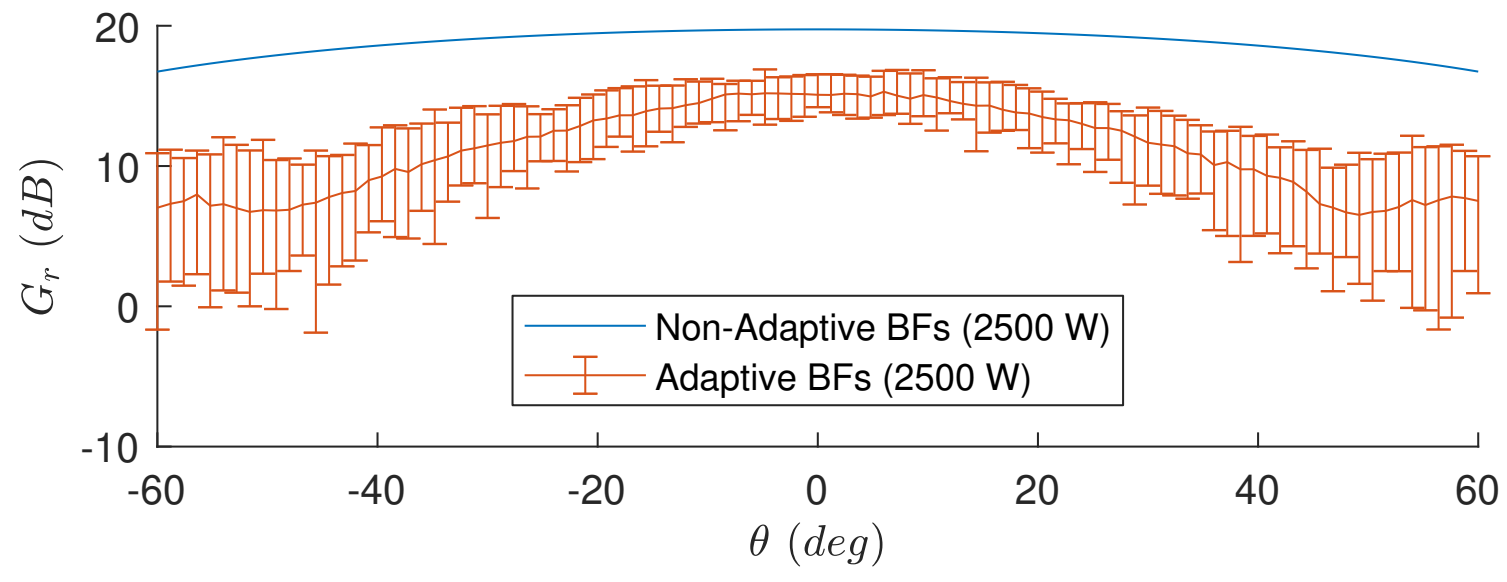

(b) $G_{r}$ vs. Azimuth Scan Angle

Figure 2.14: (a) Effective Isotropic Radiated Power (EIRP) and (b) Receive Gain $\left(G_{r}\right)$ plotted against azimuth beam angle for the non-adaptive and adaptive beamformers for the ALSTAR array configuration of Fig. 2.1(b). The adaptive results show the median EIRP and $G_{r}$ with error bars indicating the minimum and maximum values across initializations. 


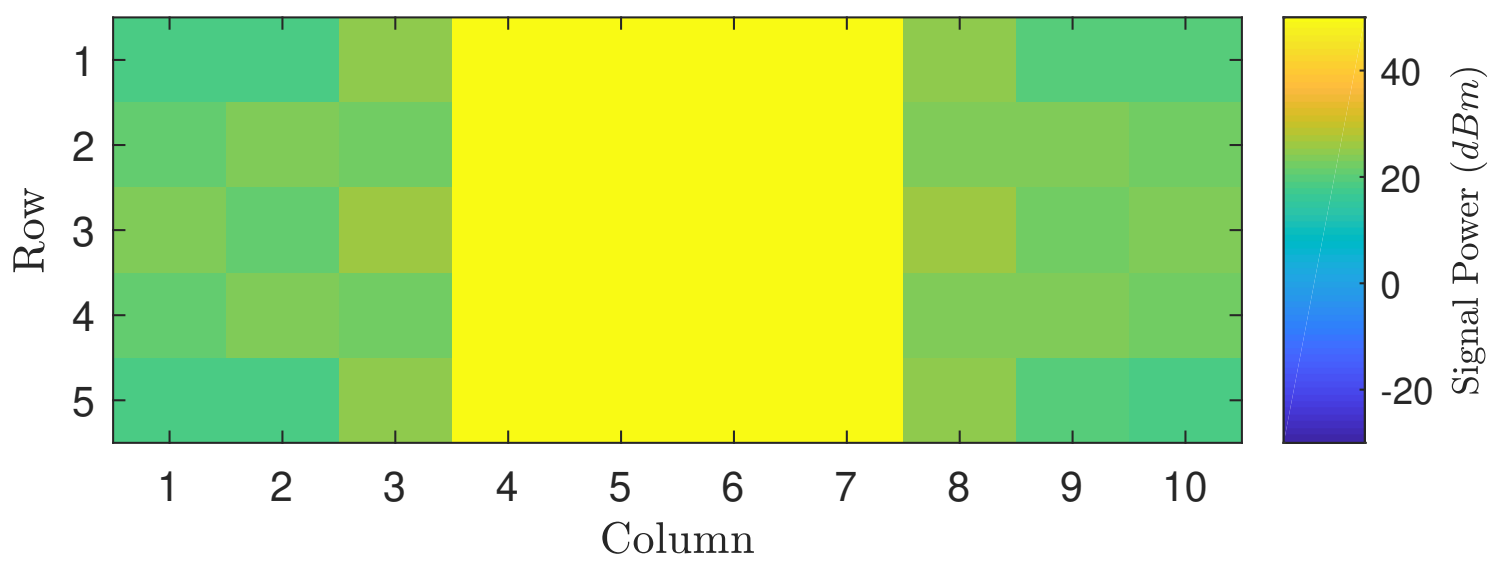

(a) Tx/Rx Element Power - Non-Adaptive Tx Beamformer

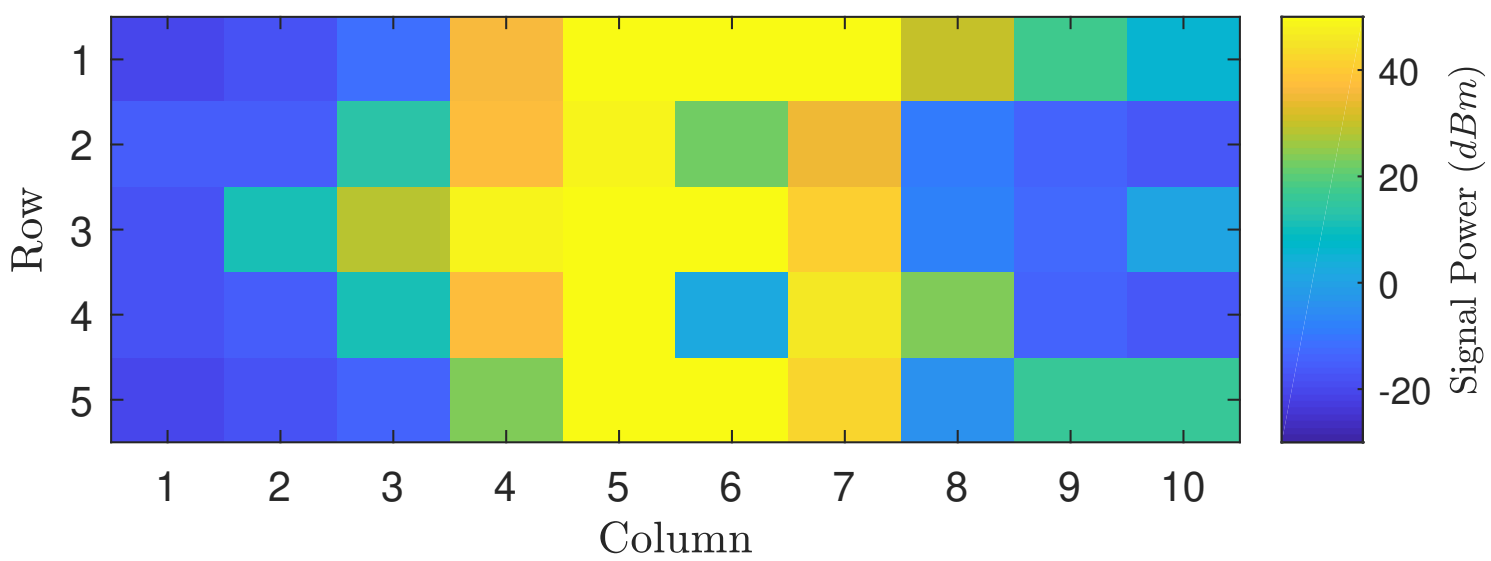

(b) Tx/Rx Element Power - Adaptive Tx Beamformer

Figure 2.15: Comparison of the transmitted power at each element and the incident power at each receive element between (a) the non-adaptive transmit beamformer and (b) the adaptive transmit beamformer for the aperture partition shown in Fig. 2.1(b) with $P_{t}=2500 \mathrm{~W}$. 


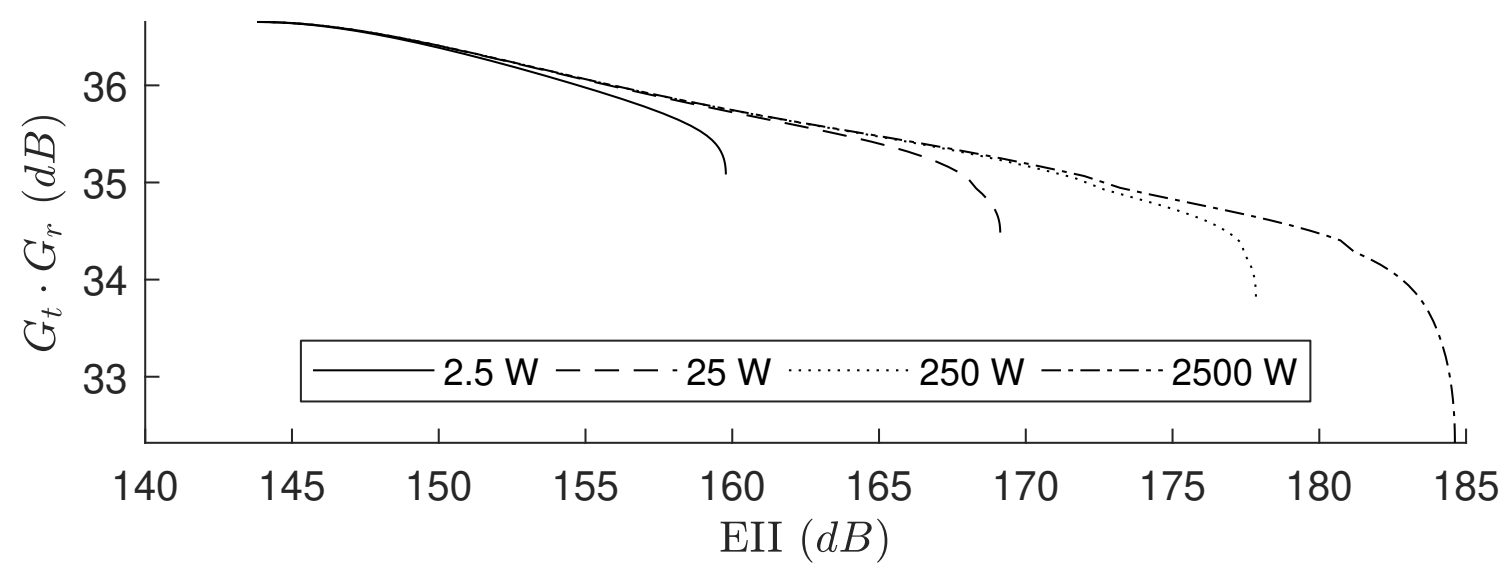

(a) Total Array Gain vs. EII

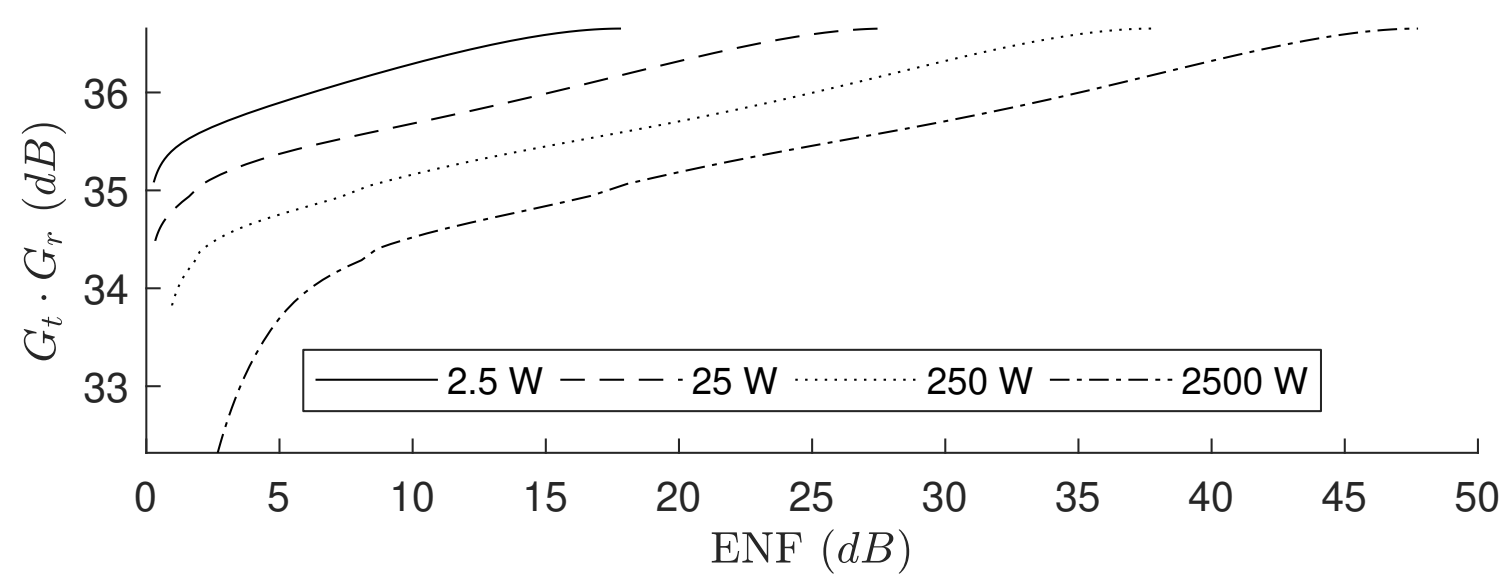

(b) Total Array Gain vs. ENF

Figure 2.16: (a) Total Array Gain versus EII and (b) Total Array Gain versus ENF, at $\theta=30^{\circ}$ for the array configuration of Fig. 2.1(a), at $P_{t}=2.5$ $\mathrm{W}, 25 \mathrm{~W}, 250 \mathrm{~W}$, and $2500 \mathrm{~W}$. The regularization coefficient $\gamma$ varied from -200 to $30 \mathrm{dBm}$. 



\section{Chapter 3}

Narrowband Direction-of-Arrival

\section{Estimation with Aperture-Level}

\section{Simultaneous Transmit and}

\section{Receive Digital Phased Arrays}

\subsection{Introduction}

Phased arrays have demonstrated incredible flexibility and capability in a wide vari-

ety of sensing and communications tasks, even as implementation size, weight, power, 
and cost have decreased. For these reasons, phased arrays are finding applications in many challenging scenarios, including self-driving cars, weather radars, joint communications and sensing apertures, and future cellular networks. Many of these scenarios could benefit from in-band simultaneous transmit and receive (STAR) (i.e. transmitting and receiving at the same time in the same frequency band). The fundamental difficulty in achieving STAR is suppressing the effects of the self-interference (SI) between the transmit and receive channels. Both Sabharwal et al. [134] and Kolodziej et al. 4] catalog a large number of techniques for mitigating SI in STAR platforms. While many approaches include specialized antennas, circulators, and analog cancellation hardware, this work studies the Aperture-Level Simultaneous Transmit and Receive (ALSTAR) phased array architecture which achieves STAR via adaptive digital beamforming and digital self-interference cancellation (SIC) only [1].

With respect to radar operation, STAR avoids the need to range gate the received signal, eliminating blind spots near the radar. By extending the coherent integration interval, STAR enables low-power continuous illumination which can increase the performance of radar systems operating under strict power constraints. Additionally, low-power long-integration illumination can be important in scenarios where the radar system must remain unobserved by other sensors [135].

In this paper we explore the performance of an ALSTAR array in a narrowband direction-of-arrival (DOA) estimation task. Using the Fisher Information on the 
mean-squared error (MSE) of the estimated DOA as our objective function, we propose a beamforming technique that maximizes DOA resolution in the presence of SI. We also explore whether optimizing the partitioning of the phased array aperture into transmit and receive antennas can significantly improve the Bayesian Information, which is the inverse of the Bayesian Cramér-Rao Lower Bound (BCRB) and bounds the performance of the aray over the scan space.

\subsubsection{DOA Related Work}

The problem of DOA estimation from array sample data has been thoroughly explored in the literature, but the traditional DOA estimation problem generally only deals with the propagation of signals from a source or a reflector that has already been illuminated. Many algorithms have been developed to solve the DOA estimation problem, including MUSIC [136], ESPRIT [137, 138], and expectation maximization (EM) [139].

Stoica and Nehorai present derivations of the maximum-likelihood (ML) DOA estimators and the corresponding Cramér-Rao Lower Bounds (CRLBs) on DOA estimation error under deterministic and random signal models for the signal-of-interest [140]. Smith presents results for the CRLB on the angular resolution of multiple impinging signals under the assumption that the complex amplitudes of the signals must also 
be estimated [141]. Li and Compton present ML estimators and the corresponding CRLBs for DOA estimation for multiple narrowband signals with varying levels of knowledge about the signals [142]. Pesavento and Gershman propose an ML DOA estimation algorithm that assumes uncorrelated noise at different powers for the elements of a direction finding array and presents the CRLBs for single and multiple sources with deterministic and stochastic priors [143].

Nguyen and Van Trees demonstrated via simulation that the Chazan-Ziv-Zakai Bound on DOA estimation error was tighter than the BCRB and Weiss-Weinstein Bound (WWB) for the case when only one plane wave impinges on the array. They also suggested that below the signal-to-noise-ratio (SNR) threshold, an EM algorithm outperforms MUSIC-type algorithms, while the MUSIC and EM algorithms perform similarly in the high SNR region [144]. Bell et al. described how the Extended ZivZakai Bound (EZZB) may be applied to the DOA estimation problem for a receive array of any geometry [145]. The EZZB better matches the MSE performance of the ML estimator in low SNR regimes.

Athley applied the Method of Interval Estimation (MIE) technique to predict the estimated DOA MSE for single- and several-source scenarios with both random and nonrandom models for the signal-of-interest [146]. Richmond describes how the MIE may be applied to scenarios where the receiver noise covariance matrix must also be 
estimated for adaptive DOA estimation and how the estimation error on the covariance matrix contributes to overall DOA estimation error [147]. Kantor et al. propose that the MIE method DOA MSE estimators could be used to make online decisions between the ML and MAP estimates in the case of a poorly chosen prior [148].

Beyond calculating the fundamental angular resolution limits of arrays, a number of authors have considered how the placement of antennas in the array affect these limits. Athley proposed that the threshold between asymptotic and low-SNR performance regions for the WWB on DOA estimation MSE be used as a metric for the placement of elements in a sparse linear receive array, including both continuous and discrete antenna positions [149]. Gavish and Weiss proposed an array geometry optimization technique that minimized a lower bound on the weighted Euclidean distance between steering vectors in an effort to reduce the large-angle errors common in low-SNR and low sample size scenarios [150]. Mirkin and Sibul explored the coupling in the CRLB for simultaneous azimuth and elevation DOA estimation and gave the conditions on array geometry that provide uncoupled azimuth and elevation estimation [151]. Gazzah and Marcos also discussed how array geometry is incorporated into the CRLB and explored how the angle between the arms of a V-shaped array affected the CRLB [152]. Dogandzic and Nehorai presented the CRLB for target range, velocity, and DOA for an array of arbitrary shape that is illuminating the target with pulses. They then considered the effect of several different array geometries on the CRLB [153]. Lange and Yang described a method of subarray, amplitude taper, and 
element position optimization that minimizes the CRLB on DOA estimation error under sidelobe level and minimum element separation constraints [154]. Oktel and Moses described an approach to array geometry design that minimizes the expected CRLB or maximizes the expected Fisher Information, similar to the approach in this work [155]. However, their work considered a receive-only array with element positions that are continuously variable, where our work considers an aperture that must simultaneously illuminate the target and sense the reflected wave with antennas at fixed positions within a grid.

\subsubsection{STAR Related Work}

In an attempt to achieve STAR, many authors have proposed circulator-like devices that isolate the transmit and receive channels for a single antenna [156, 157, 158, 159]. Melzer et al. described a method of near-field reflection interference cancellation for frequency modulated continuous wave (FMCW) radars with one transmit and one

receive channel using a measured copy of the transmitted signal and an analog delay line [160]. Melzer et al. also extended their work to adaptively calculate least-squares estimates of the required cancellation parameters [161]. Venkatamuni et al. proposed an adaptive, digitally-controlled, analog SIC system to supplement the isolation created by a circulator in a single antenna FMCW radar [162]. Fitz et al. described 
a method of SI suppression via circulators, analog cancellation, and digital cancellation to enable out-of-band sensing in close proximity to high power radar systems [57]. They achieved $106 \mathrm{~dB}$ of isolation in their narrowband experiment. Ku et al. demonstrated approximately $50 \mathrm{~dB}$ of transmit/receive isolation between separated 8element transmit and receive arrays in a FMCW millimeter-wave phased array radar [163]. The isolation was achieved by separating the transmit and receive antennas, electronics, power supplies, and ground planes, and by using internal shielding.

The ALSTAR architecture studied in this paper was originally presented in [1, and the performance of an 8-element linear ALSTAR array was documented in [6]. An advanced adaptive beamforming technique to maximize effective isotropic isolation (EII) was proposed and simulated in Chapter 2. Previous work also explored the performance of the ALSTAR architecture in full-duplex communications [129] and imaging tasks [127, 128], though the beamforming techniques there were based on simpler models of the ALSTAR architecture and did not directly optimize the performance metrics defined for the array. Here, we extend the imaging related work presented in [127] and [128] by applying the iterative optimization approach presented in Chapter 2 to the Fisher Information of the DOA estimate. We also explore the impact of the transmit/receive aperture partition, including larger array sizes than are found in our previous work. 
Section 3.2 outlines the ALSTAR array architecture and corresponding statistical signal model. Section 3.3 introduces the Fisher Information of the estimated DOA of a point target in the far-field for an ALSTAR array and proposes a method of optimizing the transmit and receive beamformers to maximize the Fisher Information. Section 3.4 extends the Fisher Information to the Bayesian Information to account for the fact that a target may appear at any point in the field of view of the array. In Section 3.5, we apply a Genetic Algorithm to the problem of optimizing the transmit/receive aperture partition. Section 3.6 discusses the results of the beamforming and aperture partition optimization techniques for a 10-element linear and a $5 \times 10$ element rectangular ALSTAR array. Sections 3.7 concludes and discusses potential future directions of inquiry.

\subsection{ALSTAR Array Signal Model}

This work is based on the ALSTAR phased array architecture as discussed in Chapter 2. which was extended from the original presentation in [1]. Fig. 3.1(a) defines the symbols involved in the signal flow diagram of the ALSTAR architecture given in Fig. 3.1(b), In this work we consider an array with $J+K$ antennas that can each be dynamically reconfigured to transmit or receive. Specifically, we assume that $J$ antennas are configured to transmit and $K$ antennas are configured to receive. The

array transmits a complex narrowband signal $x \in \mathbb{C}$ with $E\left[|x|^{2}\right]=1$. This signal 


\begin{tabular}{cl} 
Symbol & Definition \\
\hline$x$ & Transmitted Signal \\
$\mathbf{b}_{t}$ & Transmit Beamformer \\
$\mathbf{n}_{t}$ & Transmitter Noise \\
$\mathbf{t}$ & Transmitted Signal + Noise \\
$\mathbf{n}_{o}$ & Observation Noise \\
$\mathbf{H}_{o}$ & Observation Channel \\
$\mathbf{o}$ & Observed Signals \\
$\mathbf{b}_{c}$ & Cancellation Filter \\
$\mathbf{M}$ & Mutual Coupling Channel \\
$\mathbf{s}$ & Signal of Interest \\
$\mathbf{n}_{r}$ & Receiver Noise \\
$\mathbf{r}$ & Received Signals \\
$\mathbf{b}_{r}$ & Receive Beamformer \\
$y$ & Received Beamformed Signal \\
$y^{\prime}$ & Isolated Received Signal \\
\hline
\end{tabular}

(a)

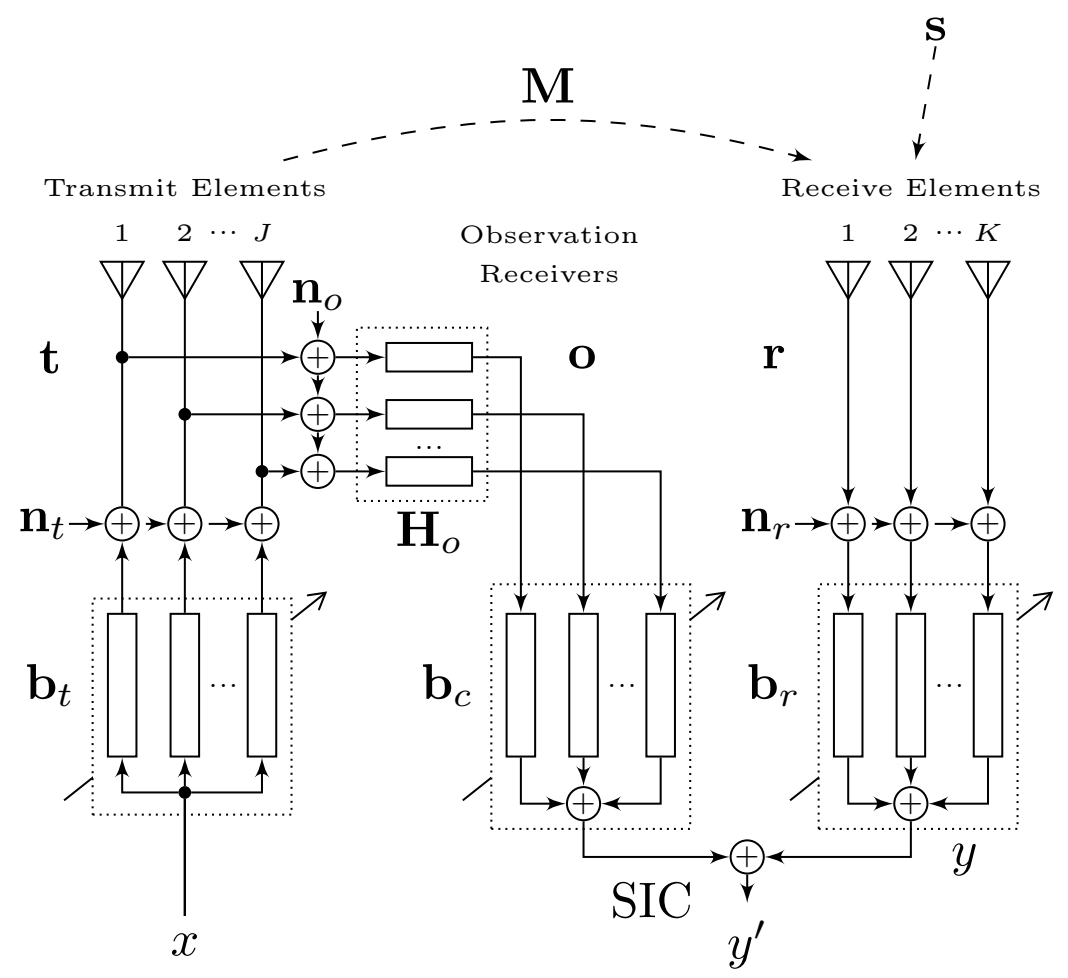

(b)

Figure 3.1: (a) List of symbols and their corresponding definitions. (b) ALSTAR architecture signal flow diagram, both from [1] and Chapter 2. 
is passed through the transmit beamformer $\mathbf{b}_{t} \in \mathbb{C}^{J \times 1}$ to the transmit antennas. We assume that each transmit channel is a linear additive white Gaussian noise (AWGN) channel with fixed dynamic range $\eta_{t}$. Therefore, the signals transmitted by the antennas are described by

$$
\mathbf{t}=\mathbf{b}_{t} x+\mathbf{n}_{t}
$$

where $\mathbf{n}_{t} \sim \mathcal{C N}\left(\mathbf{0}, \eta_{t}^{-1} \operatorname{Diag}\left(\mathbf{b}_{t} \mathbf{b}_{t}^{\mathrm{H}}\right)\right)$.

We model the propagation of the narrowband signal to and from the scene as a phase shift and a free-space path-loss, assuming that the scene is in the far field of the array, which implies that the transmitted and reflected waves can be modeled as plane waves. We assume that the scene is a point target in free space at a distance $d$ meters from the array. Mathematically, for the $r$ th receiving element and $t$ th transmitting element, the propagation is modeled by the $(r, t)$ th element of the matrix

$$
\mathbf{H}(\phi, \theta)=\frac{1}{d^{2}} \mathbf{q}_{r}(\phi, \theta) \mathbf{q}_{t}^{\mathrm{H}}(\phi, \theta)
$$

where $\mathbf{q}_{t}$ and $\mathbf{q}_{r}$ are the transmit and receive array manifold vectors,

$$
\begin{aligned}
& \mathbf{q}_{t}(\phi, \theta)=e^{-j \frac{2 \pi}{\lambda}\left(\mathbf{x}_{t} \cos \phi \sin \theta+\mathbf{y}_{t} \sin \phi \sin \theta\right)}, \\
& \mathbf{q}_{r}(\phi, \theta)=e^{j \frac{2 \pi}{\lambda}\left(\mathbf{x}_{r} \cos \phi \sin \theta+\mathbf{y}_{r} \sin \phi \sin \theta\right)} .
\end{aligned}
$$




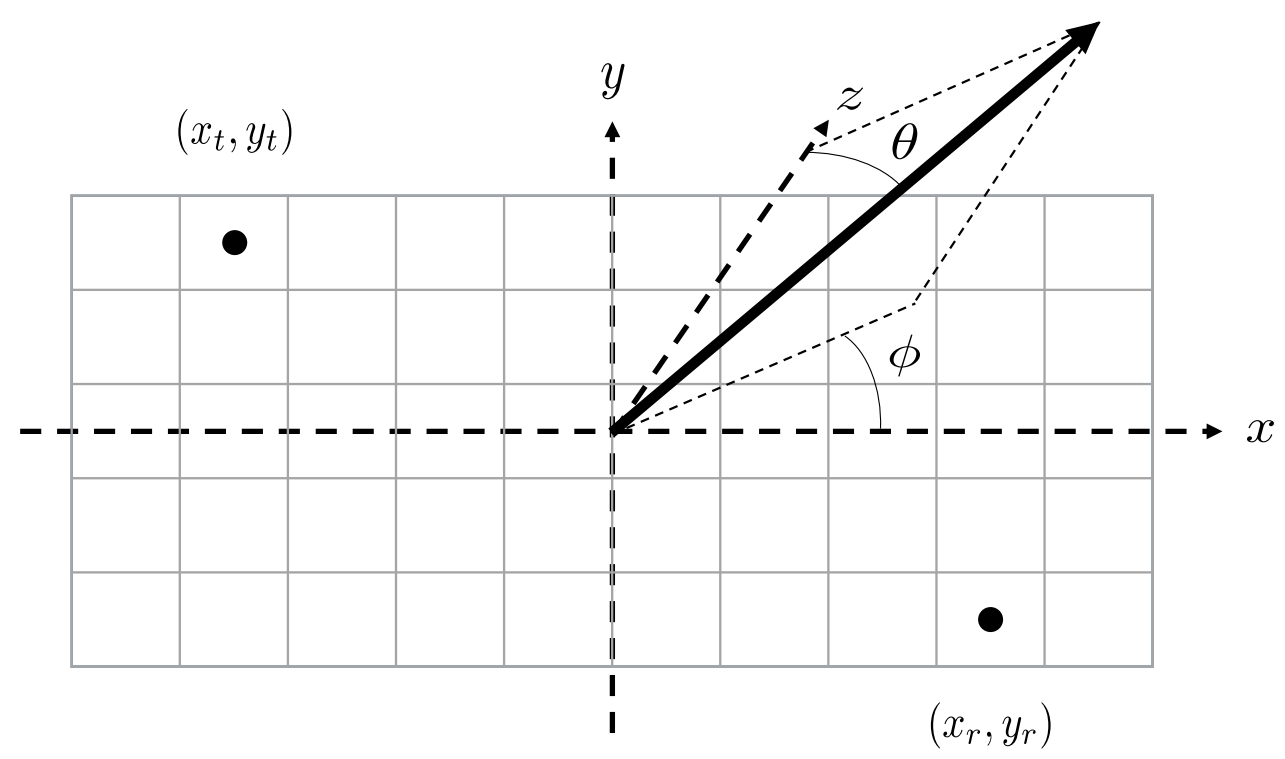

Figure 3.2: Definition of the coordinate system used for element positions and beam scan angles in this work.

As shown in Fig. 3.2, $x_{t}$ is the $\mathrm{x}$-coordinate of the $t$ th transmitting antenna, $y_{t}$ is the $\mathrm{y}$ coordinate of the $t$ th transmitting antenna, $x_{r}$ is the x-coordinate of the $r$ th receiving antenna, $y_{r}$ is the y-coordinate of the $r$ th receiving antenna, $\phi$ is the angle of the main beam in the $x y$-plane from the positive $x$-axis, and $\theta$ is the angle between the main beam and array normal (i.e. the positive $z$-axis). We have chosen to use opposite signs in the exponents of $\mathbf{q}_{t}$ and $\mathbf{q}_{r}$ to account for the fact that the receive beamformer is applied with a Hermitian transpose. In addition to the array-scene-array propagation path, there is a direct mutual coupling between the transmit and receive antennas of the array. We model this mutual coupling with matrix $\mathbf{M} \in \mathbb{C}^{K \times J}$ as shown in Fig. 3.1b, where the $(r, t)$ th element describes the narrowband coupling from the $t$ th 
transmitting antenna to the $r$ th receiving antenna. As in Chapter 2, we use an Ansys HFSS model of an array of patch antennas similar to those described in [133] with $\lambda / 2$ spacing when the array is operated at $f=2.42 \mathrm{GHz}$. In practice, the coupling channel would have to be estimated using a channel sounding technique [77, 178, 131] in order to account for mutual coupling caused by reflectors in the environment near the array. However, because this work is concerned with the calculation of lower bounds on DOA estimation performance for the ALSTAR architecture and determining optimal array configurations prior to system deployment, we consider the assumption of perfect coupling channel knowledge to be reasonable. Given these channel models, the signal received at each antenna is

$$
\mathbf{r}=\mathbf{H}\left(\mathbf{b}_{t} x+\mathbf{n}_{t}\right)+\mathbf{M}\left(\mathbf{b}_{t} x+\mathbf{n}_{t}\right)
$$

We assume that the transmitted noise power is sufficiently attenuated by the two-way path loss to and from the scene being imaged that it falls well below the other noise terms and can be ignored, i.e.

$$
\mathbf{r}=\mathbf{H b}_{t} x+\mathbf{M}\left(\mathbf{b}_{t} x+\mathbf{n}_{t}\right)
$$

Like with the transmit channels, we assume that each receive channel is a linear AWGN channel with fixed dynamic range $\eta_{r}$, where the incident power at each element sets the noise floor. The received signals $\mathbf{r}$ and additive receiver noise $\mathbf{n}_{r}$ pass through 
the receive beamformer $\mathbf{b}_{r}$ to obtain the receive beamformed signal,

$$
y=\mathbf{b}_{r}^{\mathrm{H}}\left[\mathbf{H b}_{t} x+\mathbf{M}\left(\mathbf{b}_{t} x+\mathbf{n}_{t}\right)+\mathbf{n}_{r}\right],
$$

where $\mathbf{n}_{r} \sim \mathcal{C N}\left(\mathbf{0}, \eta_{r}^{-1} E\left[\operatorname{Diag}\left(\mathbf{r r}^{\mathrm{H}}\right)\right]+\sigma_{r}^{2} I\right) . \sigma_{r}^{2}$ represents the fixed thermal noise power of each individual receive channel. The desired signal $\mathbf{b}_{r}^{H} \mathbf{H} \mathbf{b}_{t} x$ that contains information about the scene being imaged is corrupted by the SI and noise terms in $y$. Therefore, in order to isolate the desired signal from the SI and noise, the ALSTAR architecture implements digital SIC. Traditionally, SIC involves using knowledge of the transmitted signal $x$ and the mutual coupling channel $\mathbf{M}$ to cancel the signal component of the $\mathrm{SI} \mathbf{b}_{r}^{\mathrm{H}} \mathbf{M b}_{t} x$. However, the component of the SI due to the transmitted noise $\mathbf{n}_{t}$ is also significant. In order to address this difficulty, the ALSTAR architecture observes the transmitted signal and noise in each transmit channel via the unused receive channel behind each transmitting element. Recall that each antenna in an ALSTAR array can be reconfigured dynamically as a transmit or receive element. This is achieved via the switching scheme shown in Fig. 3.3. It is possible to effectively measure the transmitted noise because the dynamic range of the transmit channel is typically much smaller than that of the receive channel (e.g. $\left.\eta_{t}=45 d B \ll \eta_{r}=70 d B\right)$ due to high transmit power amplifier noise and distortion. The model for the observed signals is

$$
\mathbf{o}=\mathbf{H}_{o}\left(\mathbf{t}+\mathbf{n}_{o}\right),
$$




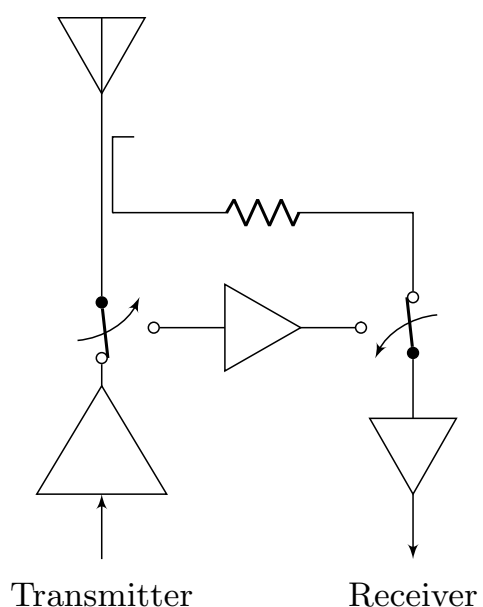

Figure 3.3: Diagram of the switching system behind each element in an ALSTAR array [1]. When configured as shown, the antenna is connected to the transmit RF chain and the receiver is connected to a reference measurement tap near the antenna through an attenuator. In this way, the typically unused receiver is used to observe the transmitted signal and noise when the antenna is transmitting. When configured to receive, the switches connect the antenna to the receive $\mathrm{RF}$ chain via a low-noise amplifier.

where $\mathbf{H}_{o}$ is a diagonal channel matrix that accounts for the scaling of the attenuators and $\mathbf{n}_{o}$ is the independent AWGN observation receiver noise, which has distribution

$$
\begin{aligned}
\mathbf{n}_{o} & \sim \mathcal{C N}\left(\mathbf{0}, \eta_{r}^{-1}\left(1+\eta_{t}^{-1}\right) \operatorname{Diag}\left(\mathbf{b}_{t} \mathbf{b}_{t}^{\mathrm{H}}\right)\right) \\
& \approx \mathcal{C N}\left(\mathbf{0}, \eta_{r}^{-1} \operatorname{Diag}\left(\mathbf{b}_{t} \mathbf{b}_{t}^{\mathrm{H}}\right)\right)
\end{aligned}
$$

where we have assumed that $\eta_{t}$ is large enough that $1+\eta_{t}^{-1} \approx 1$. Again this noise represents the fixed dynamic range of the observation channels. From Fig. 3.1b we see that the final isolated signal is produced by combining the output of the receive beamformer $\mathbf{b}_{r}$ and a cancellation filter $\mathbf{b}_{c}$, which combines the observed signals to 
create a SIC signal. Therefore, we model the final isolated received signal

$$
\begin{aligned}
y^{\prime}= & y+\mathbf{b}_{c}^{\mathrm{H}} \mathbf{o} \\
= & \mathbf{b}_{r}^{\mathrm{H}}\left[\mathbf{H} \mathbf{b}_{t} x+\mathbf{M}\left(\mathbf{b}_{t} x+\mathbf{n}_{t}\right)+\mathbf{n}_{r}\right] \\
& +\mathbf{b}_{c}^{\mathrm{H}} \mathbf{H}_{o}\left(\mathbf{b}_{t} x+\mathbf{n}_{t}+\mathbf{n}_{o}\right) .
\end{aligned}
$$

Notice that choosing

$$
\mathbf{b}_{c}=-\mathbf{H}_{o}^{-1} \mathbf{M}^{\mathrm{H}} \mathbf{b}_{r}
$$

results in

$$
y^{\prime}=\mathbf{b}_{r}^{\mathrm{H}}\left[\mathbf{H b}_{t} x-\mathbf{M n}_{o}+\mathbf{n}_{r}\right] .
$$

Essentially, digital SIC via the ALSTAR architecture has replaced the SI signal and noise terms with a virtually coupled observer noise term. This is an improvement over traditional digital SIC (which would only have canceled the the SI signal $\mathbf{b}_{r}^{\mathrm{H}} \mathbf{M} \mathbf{b}_{t} x$ ) because the power of the SI noise component $\mathbf{b}_{r}^{\mathrm{H}} \mathbf{M} \mathbf{n}_{t}$ is much greater than the power of the observer noise component $-\mathbf{b}_{r}^{\mathrm{H}} \mathbf{M n}_{o}$, because $\eta_{t} \ll \eta_{r}$.

We then define the residual noise covariance matrix in the receive channel (after SIC) 


$$
\begin{aligned}
\mathbf{M}_{b r}= & E\left[\left(-\mathbf{M n}_{o}+\mathbf{n}_{r}\right)\left(-\mathbf{M n}_{o}+\mathbf{n}_{r}\right)^{\mathrm{H}}\right] \\
= & \mathbf{M} E\left[\mathbf{n}_{o} \mathbf{n}_{o}^{\mathrm{H}}\right] \mathbf{M}^{\mathrm{H}}+E\left[\mathbf{n}_{r} \mathbf{n}_{r}^{\mathrm{H}}\right] \\
= & \eta_{r}^{-1} \mathbf{M} \operatorname{Diag}\left(\mathbf{b}_{t} \mathbf{b}_{t}^{\mathrm{H}}\right) \mathbf{M}^{\mathrm{H}} \\
& +\eta_{r}^{-1} E\left\{\operatorname{Diag}\left[\mathbf{M}\left(\mathbf{b}_{t} x+\mathbf{n}_{t}\right)\left(\mathbf{b}_{t} x+\mathbf{n}_{t}\right)^{\mathrm{H}} \mathbf{M}^{\mathrm{H}}\right]\right\} \\
& +\sigma_{r}^{2} I \\
= & \underbrace{\eta_{r}^{-1} \mathbf{M} \text { Diag }\left(\mathbf{b}_{t} \mathbf{b}_{t}^{\mathrm{H}}\right) \mathbf{M}^{\mathrm{H}}}_{\text {SIC Observer Noise }} \\
& +\underbrace{\eta_{r}^{-1} \text { Diag }\left(\mathbf{M b}_{t} \mathbf{b}_{t}^{\mathrm{H}} \mathbf{M}^{\mathrm{H}}\right)}_{\text {Rx Noise due to Tx Signal SI }} \\
& +\underbrace{\eta_{r}^{-1} \eta_{t}^{-1} \text { Diag }\left[\mathbf{M} \text { Diag }\left(\mathbf{b}_{t} \mathbf{b}_{t}^{\mathrm{H}}\right) \mathbf{M}^{\mathrm{H}}\right]}_{\text {Rx Noise due to Tx Noise SI }} \\
& +\underbrace{\sigma_{r}^{2} I}_{\text {Rx Thermal Noise }} \text {. }
\end{aligned}
$$

We note that the increase in noise floor due to the presence of the signal reflected from the scene is negligible, and therefore the signal term in $\mathbf{r}$ can be ignored in calculating the covariance matrix of $\mathbf{n}_{r}$. The total residual noise power in the received signal is defined

$$
\sigma_{n}^{2}=\mathbf{b}_{r}^{\mathrm{H}} \mathbf{M}_{b r} \mathbf{b}_{r}
$$

It turns out that we can use the vector identity $\mathbf{a}^{\mathrm{H}} \operatorname{Diag}\left(\mathbf{b b}^{\mathrm{H}}\right) \mathbf{a}=\mathbf{b}^{\mathrm{H}} \operatorname{Diag}\left(\mathbf{a a}^{\mathrm{H}}\right) \mathbf{b}$ 
to re-write $\sigma_{n}^{2}$ as a quadratic form in $\mathbf{b}_{t}$ instead of $\mathbf{b}_{r}$,

$$
\sigma_{n}^{2}=\mathbf{b}_{t}^{\mathrm{H}} \mathbf{M}_{b t} \mathbf{b}_{t}
$$

with the corresponding covariance matrix

$$
\begin{aligned}
\mathbf{M}_{b t}= & \underbrace{\eta_{r}^{-1} \operatorname{Diag}\left(\mathbf{M}^{\mathrm{H}} \mathbf{b}_{r} \mathbf{b}_{r}^{\mathrm{H}} \mathbf{M}\right)}_{\text {SIC Observer Noise }} \\
& +\underbrace{\eta_{r}^{-1} \mathbf{M}^{\mathrm{H}} \operatorname{Diag}\left(\mathbf{b}_{r} \mathbf{b}_{r}^{\mathrm{H}}\right) \mathbf{M}}_{\text {Rx Noise due to Tx Signal SI }} \\
& +\underbrace{\eta_{r}^{-1} \eta_{t}^{-1} \operatorname{Diag}\left[\mathbf{M}^{\mathrm{H}} \operatorname{Diag}\left(\mathbf{b}_{r} \mathbf{b}_{r}^{\mathrm{H}}\right) \mathbf{M}\right]}_{\text {Rx Noise due to Tx Noise SI }} \\
& +\underbrace{\frac{\sigma_{r}^{2}}{P_{t}} I}_{\text {Rx Thermal Noise }} .
\end{aligned}
$$

\subsection{Fisher Information of the Direction of Arrival}

\section{Estimate}

In this work, the ALSTAR array is faced with the task of illuminating a scene and locating a single point target that lies within the azimuth scan space of the array. The performance of the array is measured by the DOA angular resolution limit, quantified by the Fisher Information of the azimuthal DOA $\left(\theta_{t}\right)$ for a plane wave from the target. 
The Fisher Information is defined as

$$
\mathcal{J}\left(\theta_{t}\right)=-E_{y^{\prime} \mid \theta_{t}}\left[\frac{\partial^{2}}{\partial \theta_{t}^{2}} \ln p\left(y^{\prime} \mid \theta_{t}\right)\right]
$$

Given the signal model above, the likelihood function for the measurement $y^{\prime}$ given the true target azimuth direction of arrival $\theta_{t}$ is circular complex Gaussian,

$$
y^{\prime} \mid \theta_{t} \sim \mathcal{C N}\left(\mu\left(\theta_{t}\right), \sigma_{n}^{2}\right)
$$

with

$$
\mu\left(\theta_{t}\right)=\mathbf{b}_{r}^{\mathrm{H}} \mathbf{H}\left(\theta_{t}\right) \mathbf{b}_{t}
$$

and $\sigma_{n}^{2}$ as defined in $(3.13)$ and (3.14). Therefore, the Fisher Information expands to

$$
\begin{aligned}
\mathcal{J}\left(\theta_{t}\right) & =2 \frac{\left(\frac{\partial \mu\left(\theta_{t}\right)}{\partial \theta_{t}}\right)^{*}\left(\frac{\partial \mu\left(\theta_{t}\right)}{\partial \theta_{t}}\right)}{\sigma_{n}^{2}} \\
& =2 \frac{\mathbf{b}_{r}^{\mathrm{H}} \frac{\partial \mathbf{H}}{\partial \theta_{t}} \mathbf{b}_{t} \mathbf{b}_{t}^{\mathrm{H}} \frac{\partial \mathbf{H}^{\mathrm{H}}}{\partial \theta_{t}} \mathbf{b}_{r}}{\mathbf{b}_{r}^{\mathrm{H}} \mathbf{M}_{b r} \mathbf{b}_{r}}
\end{aligned}
$$

Notice that if $\mathbf{b}_{t}$ is held constant (recalling that $\mathbf{M}_{b r}$ is also a function of $\mathbf{b}_{t}$ ), (3.19) becomes a generalized Rayleigh quotient in $\mathbf{b}_{r}$ with a rank-one matrix in the numerator. Given the equivalent definition of $\sigma_{n}^{2}$ as a quadratic form in terms of $\mathbf{b}_{t}$, we can also write the Fisher Information as

$$
\mathcal{J}\left(\theta_{t}\right)=2 \frac{\mathbf{b}_{t}^{\mathrm{H}} \frac{\partial \mathbf{H}^{\mathrm{H}}}{\partial \theta_{t}} \mathbf{b}_{r} \mathbf{b}_{r}^{\mathrm{H}} \frac{\partial \mathbf{H}}{\partial \theta_{t}} \mathbf{b}_{t}}{\mathbf{b}_{t}^{\mathrm{H}} \mathbf{M}_{b t} \mathbf{b}_{t}}
$$


which similarly becomes a generalized Rayleigh quotient in $\mathbf{b}_{t}$ with a rank-one matrix in the numerator when $\mathbf{b}_{r}$ is held constant. In order to choose transmit and receive beamformers that minimize the uncertainty in estimating the true value of $\theta_{t}$, we must maximize the Fisher Information in terms of $\mathbf{b}_{t}$ and $\mathbf{b}_{r}$. The structure of (3.19) and 3.20 intuitively suggests that $\mathcal{J}\left(\theta_{t}\right)$ could be maximized by holding $\mathbf{b}_{r}$ constant, solving for the $\mathbf{b}_{t}$ that maximizes 3.20 , and then holding $\mathbf{b}_{t}$ constant and solving for the $\mathbf{b}_{r}$ that maximizes (3.19), alternately solving for $\mathbf{b}_{t}$ and $\mathbf{b}_{r}$ until they converge. The generalized Rayleigh quotient with rank-one matrix in the numerator has a closed-form solution [132. For 3.19 and $(3.20)$, the solutions are

$$
\begin{aligned}
& \mathbf{b}_{t}=\alpha \mathbf{M}_{b t}^{-1} \frac{\partial \mathbf{H}^{\mathrm{H}}}{\partial \theta_{t}} \mathbf{b}_{r} \text { s.t. }\left\|\mathbf{b}_{t}\right\|=P_{t}, \\
& \mathbf{b}_{r}=\beta \mathbf{M}_{b r}^{-1} \frac{\partial \mathbf{H}}{\partial \theta_{t}} \mathbf{b}_{t} \text { s.t. }\left\|\mathbf{b}_{r}\right\|=1
\end{aligned}
$$

where $\alpha$ and $\beta$ are arbitrary scale factors chosen to satisfy the magnitude constraints. Note that the magnitude constraints can be implemented by scaling because solutions to the generalized Rayleigh quotient are magnitude-invariant. Because the optimal value for each beamformer depends on the other, we alternate between solving (3.21) and 3.22 until the solutions for $\mathbf{b}_{t}$ and $\mathbf{b}_{r}$ converge. This approach is similar in form to the technique for maximizing effective isotropic isolation (EII) in the ALSTAR architecture presented in Chapter 2. Note that $\mathcal{J}\left(\theta_{t}\right)$ is bounded above for two reasons: first, the magnitude of $\mathbf{b}_{t}$ and $\mathbf{b}_{r}$ are constrained to finite lengths, and second, the minimum possible eigenvalues of $\mathbf{M}_{b r}$ and $\mathbf{M}_{b t}$ are $\sigma_{r}^{2}$ and $\sigma_{r}^{2} / P_{t}$, respectively, 
which are both greater than 0 . Each optimization of $\mathbf{b}_{r}$ and $\mathbf{b}_{t}$ makes a monotonically non-decreasing change in $\mathcal{J}\left(\theta_{t}\right)$. Therefore, the proposed alternating optimization scheme is guaranteed to converge to at least a local optimum.

\subsection{Bayesian Information of the Direction of Ar- rival Estimate}

Recall that the Fisher Information $\mathcal{J}\left(\theta_{t}\right)$ is a function of the true azimuth angle of the target $\theta_{t}$. We model $\theta_{t}$ as a uniformly-distributed random variable with prior

$$
p\left(\theta_{t}\right)= \begin{cases}\frac{1}{\theta_{r}-\theta_{l}}, & \theta_{t} \in\left[\theta_{l}, \theta_{r}\right] \\ 0, & \text { else }\end{cases}
$$

where $\theta_{l}$ and $\theta_{r}$ are the left and right bounds on the scan range of the array, respectively. The BCRB on our estimate of the DOA is given as

$$
\mathrm{BCRB}=\left\{E_{\theta_{t}}\left[\mathcal{J}\left(\theta_{t}\right)\right]-E_{\theta_{t}}\left[\frac{\partial^{2}}{\partial \theta_{t}^{2}} p\left(\theta_{t}\right)\right]\right\}^{-1},
$$

according to [164]. In words, the BCRB is a lower bound on MSE for an estimated quantity when the prior distribution of that quantity is known. In this case the BCRB bounds the MSE of the estimated azimuthal DOA of a plane wave reflected by a point 
target when illuminated by an ALSTAR array. While the derivatives in the second term of (3.24) are undefined at the limits of the prior, because the prior is uniform we assume that it contributes no information to the DOA of the target beyond the fact that the target lies within the scan space of the array. Therefore, we assume that the second term in 3.24 is zero, yielding

$$
\mathrm{BCRB}=\left\{E_{\theta_{t}}\left[\mathcal{J}\left(\theta_{t}\right)\right]\right\}^{-1}
$$

For our purposes, we deal with the inverse of the BCRB, referred to as the Bayesian Information,

$$
\begin{aligned}
\mathcal{J}_{B} & =E_{\theta_{t}}\left[\mathcal{J}\left(\theta_{t}\right)\right] \\
& \approx \frac{\Delta_{\theta}}{\theta_{r}-\theta_{l}} \sum_{n=1}^{N} \frac{\mathcal{J}\left(\Delta_{\theta}(n-1)+\theta_{l}\right)+\mathcal{J}\left(\Delta_{\theta} n+\theta_{l}\right)}{2}
\end{aligned}
$$

where we numerically evaluate the expectation of the Fisher Information over the prior using the trapezoidal rule. We choose

$$
\Delta_{\theta}=\frac{\theta_{r}-\theta_{l}}{N-1}
$$

as the angular sampling resolution for the numerical integration, where $N$ is the number of angular samples. Practically, we propose that an ALSTAR array could jointly scan its transmit and receive beams across the field of view of the array using 
the optimal beams obtained via alternately solving (3.21) and 3.22 at each of the angles sampled in (3.26) and estimate the DOA of a target from those data.

\subsection{Partition Optimization}

We showed in two previous papers that the partitioning of the array into transmit and receive elements had a significant effect on the achievable Fisher Information of the estimated cross-range position of a target [127, 128]. However, that work was done under a signal model that did not account for limited dynamic range channels and with a sub-optimal adaptive beamforming technique. In this paper, the metric to be optimized with respect to the array partition is the Bayesian Information on the estimated azimuth angle, $\mathcal{J}_{B}$. In terms of the Bayesian Information, the partitioning of the array changes the elements in the propagation matrix $\mathbf{H}$ and the coupling matrix $\mathbf{M}$, which changes the structure of $\partial \mathbf{H} / \partial \theta_{t}, \mathbf{M}_{b r}$, and $\mathbf{M}_{b t}$. A closed form solution for the optimal partitioning of the array aperture into transmit and receive elements is not obvious. However, optimizing the partitioning of the array into transmit and receive elements can naturally be posed as a binary integer optimization problem, where each digit in a binary string corresponds to a particular antenna in the array. If a given digit is 0 , then the corresponding antenna transmits, and if the digit is 1, the corresponding antenna receives. Genetic Algorithms (GAs) are a well-studied means of solving binary integer optimization problems. The GA is a technique that 


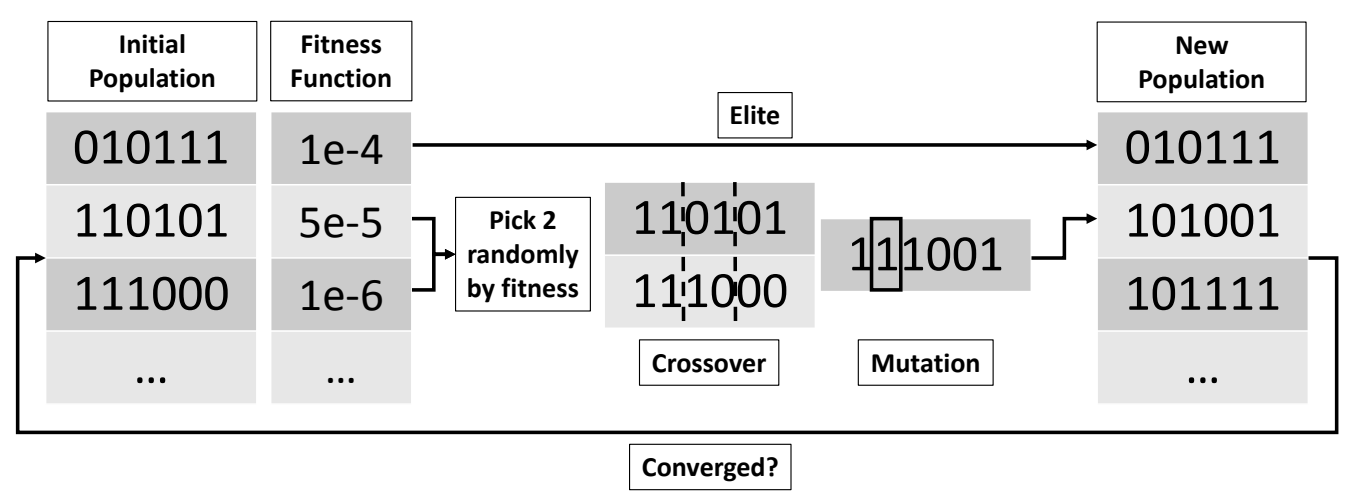

Figure 3.4: This figure illustrates the operation of the genetic algorithm, modified from [2].

effectively and naturally combines the benefits of exploring the search space and exploiting high-performance solutions found during previous iterations. As shown in Fig. 3.4, the GA begins with a randomly initialized population of potential solutions (i.e., individuals), represented by the bit strings (i.e., chromosomes) described above. The fitness of each individual is evaluated according to an objective function, which is $\mathcal{J}_{B}$ for this work. Then, pairs of individuals are chosen randomly according to their fitness to reproduce new candidate solutions. The GA randomly chooses two points to cross over the two chromosomes to form a new individual. One randomly chosen position in the resulting chromosome may then be randomly mutated (i.e., $0 \rightarrow 1$ or $1 \rightarrow 0$ ). The resulting individual is entered into a new population and this process is repeated until the new population is full. The algorithm forms new populations until the maximum fitness in the population converges or a fixed number of iterations have been reached. The GA can also retain high-performing individuals from one iteration to the next by copying them directly to the new population. These 
individuals are referred to as elites, and the use of at least one elite ensures that the maximum fitness of the population is monotonically non-decreasing. The crossover step of the GA naturally exploits the features in the chromosomes of high-performing solutions, and the mutation process helps the algorithm explore the breadth of the search space. The fact that individuals are chosen according to their fitness increases the likelihood that the resulting individual's fitness will be high. The implementation of the GA used in this work was developed with reference to [2, 165, 166]. In order to evaluate $\mathcal{J}_{B}$, recall that we must perform the beamformer optimization process across all of the sampled scan angles required by the numerical integration. Also note that $\mathcal{J}_{B}$ is bounded above because $\mathcal{J}\left(\theta_{t}\right)$ is bounded above, which implies that $\mathcal{J}_{B}$ is a satisfactory objective function for maximization.

\subsection{Results}

\subsubsection{Beamformer Optimization Results}

In order to explore the effectiveness of beamformer optimization with respect to the Fisher Information metric $\mathcal{J}$, we simulated a 10 -element uniform linear array with $\lambda / 2$ spacing, operated at $f=2.42 \mathrm{GHz}$. The elements in the array were patch antennas similar to those described in [133], and the mutual coupling of the array was modeled 
by selecting the middle row from a larger $5 \times 10$ array coupling model generated in Ansys HFSS. Fig. 3.5 plots the Fisher Information across the azimuth scan space from $\theta_{l}=-60^{\circ}$ to $\theta_{r}=60^{\circ}$ for the adaptive beamformers, calculated by iteratively solving (3.21) and (3.22). At each look angle the optimization process was initialized once with the non-adaptive beamformers and 10 times with randomly generated transmit/receive beamformer pairs scaled to meet the magnitude constraints given in (3.21) and (3.22). The non-adaptive beamformers are defined as

$$
\begin{aligned}
& \mathbf{b}_{t, n a}=\sqrt{\frac{P_{t}}{J}} \mathbf{q}_{t}(\phi, \theta), \\
& \mathbf{b}_{r, n a}=\sqrt{\frac{1}{K}} \mathbf{q}_{r}(\phi, \theta),
\end{aligned}
$$

where $\phi$ is the angle of the main beam from the positive $x$-axis in the $x y$-plane and $\theta$ is the angle between the main beam and the positive $z$-axis, as defined in Fig. 3.2 . Throughout the scan space, the adaptive beamformer optimization process produces results with reasonably consistent performance in terms of Fisher Information. It should be noted that the non-adaptive beamformers result in a Fisher Information equal to zero for the default partition. Focusing on the numerator of the Fisher Information, it can be shown that

$$
\mathbf{b}_{r, n a}^{\mathrm{H}} \frac{\partial H}{\partial \theta} \mathbf{b}_{t, n a}=j\left[a_{1}\left(\sum_{r=1}^{K} \sum_{t=1}^{J} x_{t}+x_{r}\right)+a_{2}\left(\sum_{r=1}^{K} \sum_{t=1}^{J} y_{t}+y_{r}\right)\right],
$$




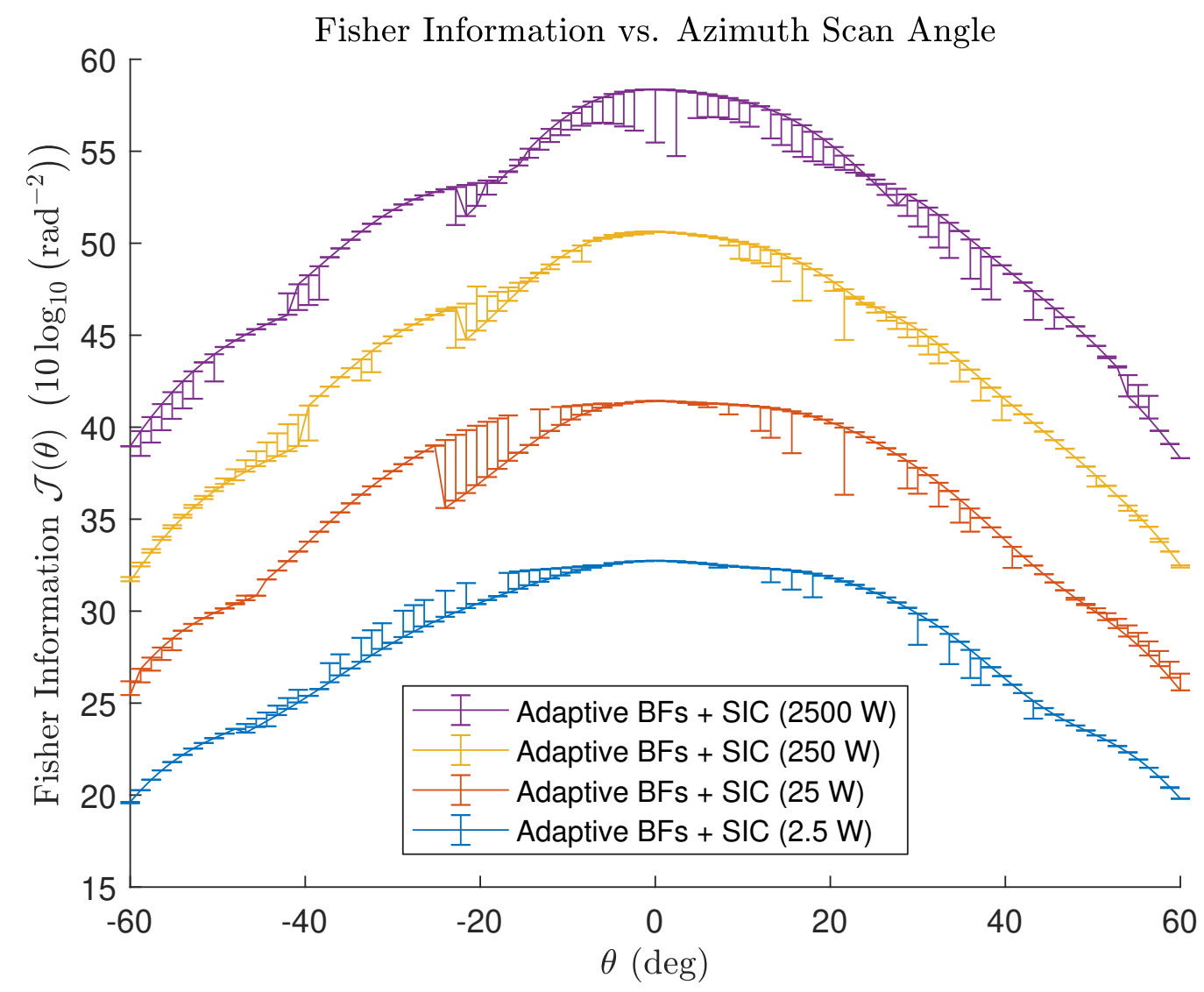

Figure 3.5: Fisher Information plotted at each scan angle for the adaptive beamformers given the aperture is in the default configuration, shown in Fig. 3.9. For the adaptive beamformers, the median Fisher Information across initializations is plotted with error bars indicating the minimum and maximum Fisher Information achieved. Results are presented for $P_{t}=2.5$ $\mathrm{W}, 25 \mathrm{~W}, 250 \mathrm{~W}$, and $2500 \mathrm{~W}$.

where $a_{1}$ and $a_{2}$ are constant with respect to the positions of the elements in the partitions of both arrays. Note that 3.30 becomes zero when the antennas are arranged symmetrically (within or across the transmit and receive partitions) about the $x$ and $y$ axes.

Fig. 3.6 plots the resulting noise power for the adaptive and non-adaptive beamformers. While the non-adaptive beamformers produce very high noise powers, the 


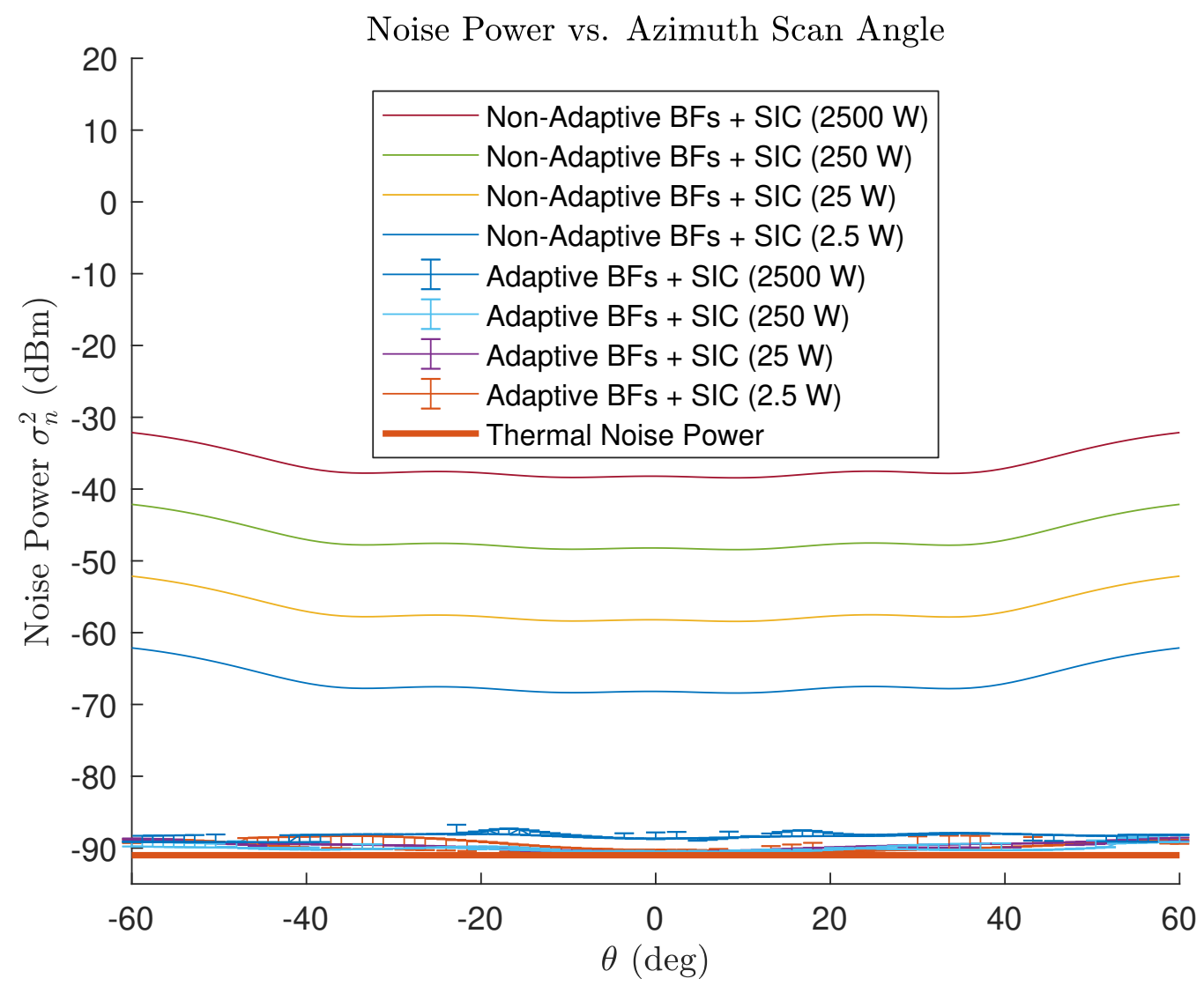

Figure 3.6: Noise power plotted at each scan angle for the adaptive and non-adaptive beamformers with the default aperture partition shown in Fig. 3.9. The median noise power across initializations is plotted for the adaptive beamformers with error bars indicating the minimum and maximum noise power achieved. Results are presented for $P_{t}=2.5 \mathrm{~W}, 25 \mathrm{~W}$, $250 \mathrm{~W}$, and $2500 \mathrm{~W}$.

adaptive beamformers are able to drive the noise power to within $1.5 \mathrm{~dB}$ of the receiver thermal noise power $\sigma_{r}^{2}$ in the median case across transmit power levels, look angles, and optimization routine initializations. These results suggest that the optimization process has successfully maximized the Fisher Information by reducing residual noise power while selecting beams that still meet the goal of localizing targets.

Fig. 3.7 shows the effective isotropic isolation (EII) achieved by the non-adaptive and 


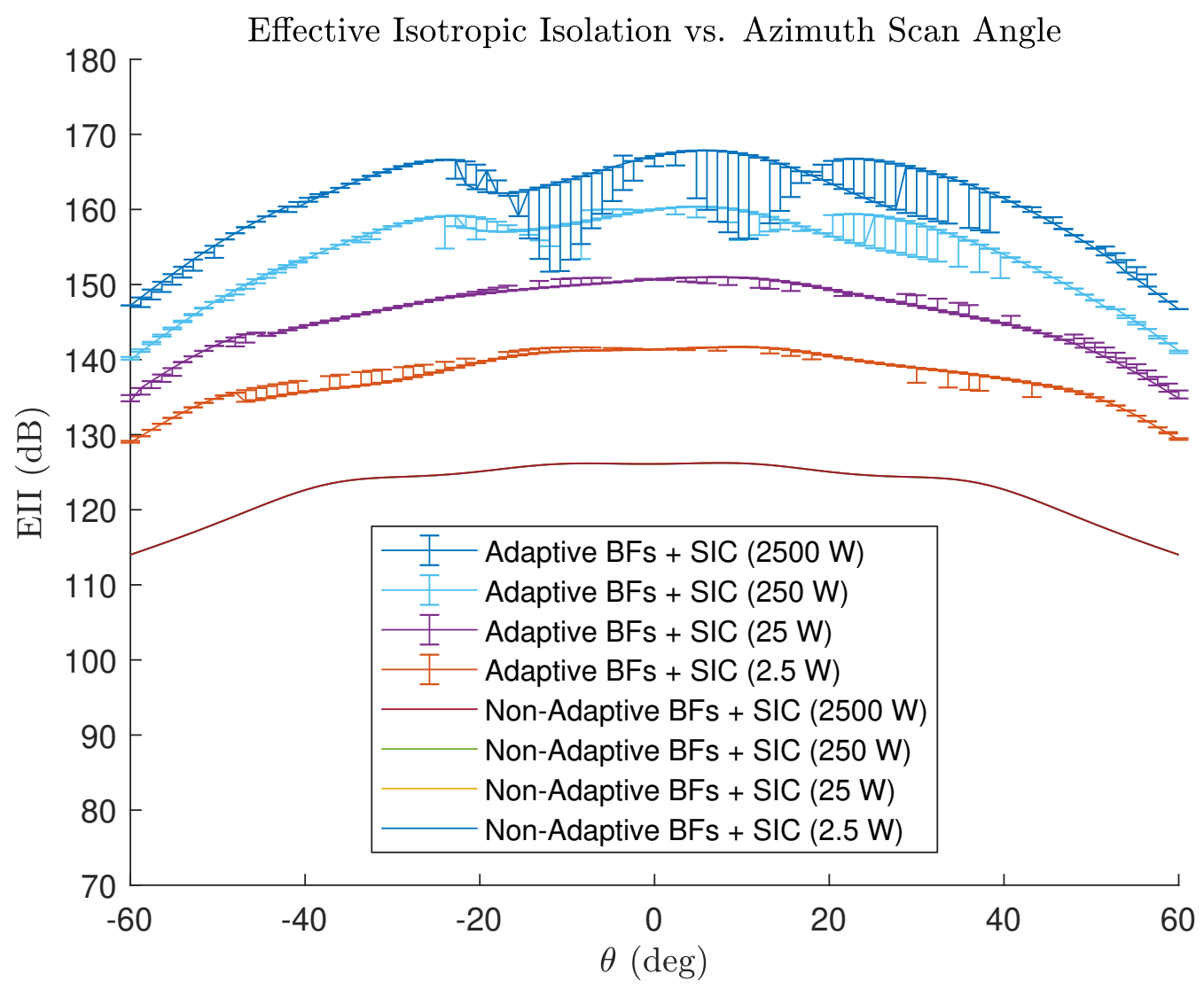

Figure 3.7: EII plotted across the azimuth scan angles for the 10-element array with the default aperture partition shown in Fig. 3.9. Results for both the non-adaptive and adaptive beamformers are presented. For the nonadaptive beamformers, the EII is almost exactly the same between transmit power levels because the effective isotropic radiated power (EIRP) scales linearly with $P_{t}$ and residual noise scales almost linearly with $P_{t}$. For the adaptive beamformers, the median EII is plotted with error bars indicating the maximum and minimum achieved across the one non-adaptive initialization and 10 random initializations of the optimization routine. While maximizing EII was not the objective, this plot shows that maximizing Fisher Information produces beamformers with high isolation that are practical for STAR. Results are presented for $P_{t}=2.5 \mathrm{~W}, 25 \mathrm{~W}, 250 \mathrm{~W}$, and $2500 \mathrm{~W}$.

adaptive beamformers, calculated according to Chapter 2, EII quantifies the ability of a STAR system to transmit with high power while maintaining high gain in the direction of interest and achieving low receiver noise power. Maximizing EII was not the goal of the optimization procedure proposed in this work. However, EII is an 
important metric in quantifying how well a phased array will perform under STAR operation. The adaptive beamformers create a high level of isolation when compared to the non-adaptive beamformers across most of the scan angles, suggesting that the proposed optimization procedure has arrived at practical solutions.

\subsubsection{Partition Optimization Results}

We also explored how partitioning the array into transmit and receive apertures affected the Bayesian Information. We began by considering all the $2^{10}-2=1022$ possible partitionings of the 10-element array that have at least one transmit and one receive element. For each of the partitions, the beamformers were optimized across the scan space and the Bayesian Information was calculated. The cumulative distribution function $(\mathrm{CDF})$ of the resulting Bayesian Information is plotted in Fig. 3.8 for each total power level, $P_{t}=2.5 \mathrm{~W}, 25 \mathrm{~W}, 250 \mathrm{~W}$, and $2500 \mathrm{~W}$. The Bayesian Information of the default partition with adaptive beamformers is indicated by the vertical black line. The GA partition optimization described in Section 3.5 was run five times on each power level with a population of 48 individuals, a crossover probability of 0.8 , a mutation probability of 0.04 , and one elite. The algorithm was terminated when $90 \%$ of the population had the same fitness as the highest performing individual or when the algorithm had gone through 1000 generations. The Bayesian Information of the partitions returned by the GA are plotted as vertical red dashed lines in Fig. 3.8. 

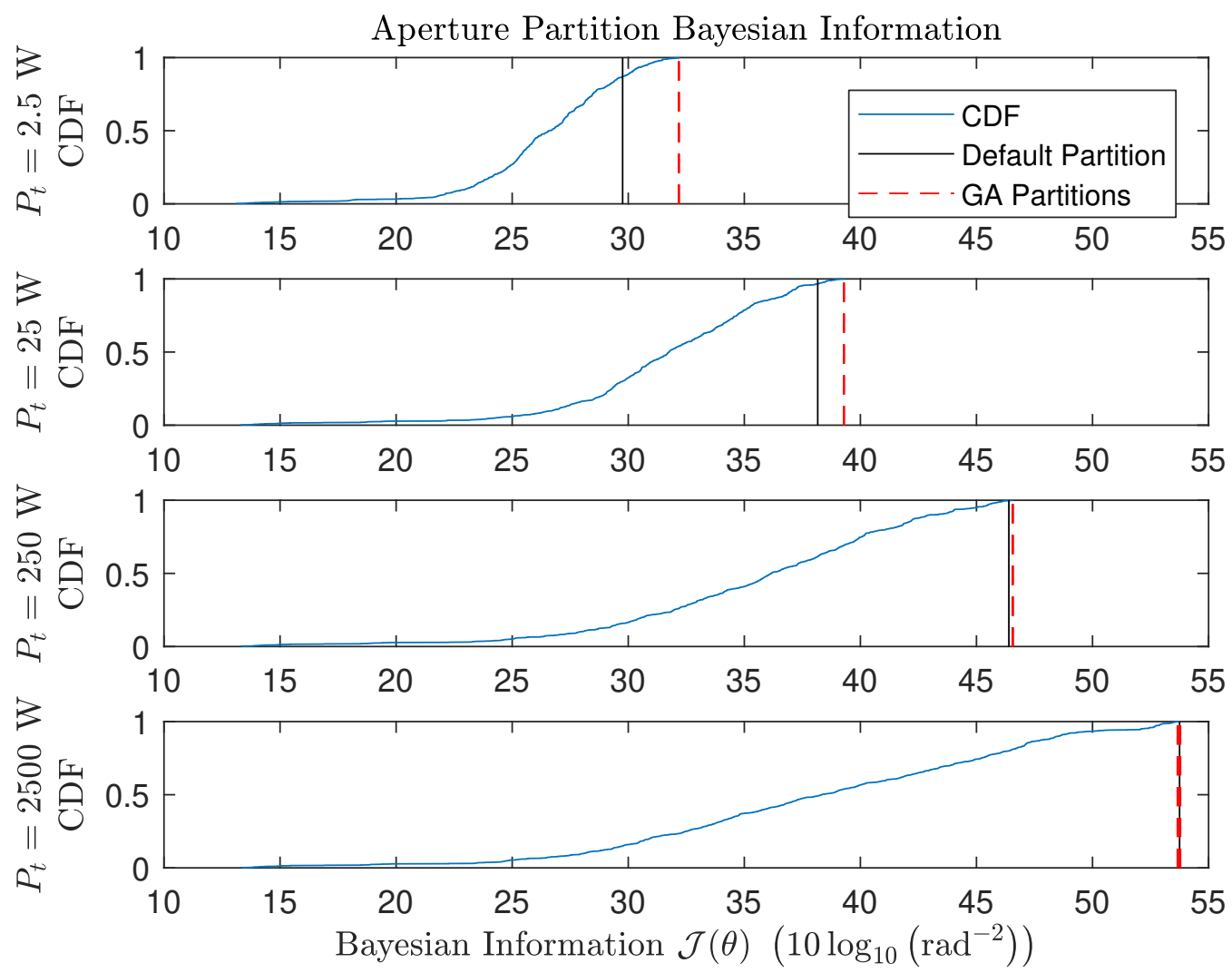

Figure 3.8: Bayesian Information distribution of the possible valid transmit/receive partitions for the 10-element array. The performance of the default partition with adaptive beamformers is plotted as a vertical black dashed line, and the performance of the GA optimized partitions are plotted as vertical red dashed lines. Five trials of the GA optimization were run at each power level.

The default partition and optimal partition for each power level are shown in Fig. 3.9.

Each trial of the GA at a given power level produced the same partition or a partition of the same geometry with transmit and receive antennas swapped. Therefore, we have confidence in the effectiveness of the GA as a tool for finding the optimal transmit/receive partitions. The median gain (across GA trials) in Bayesian Information of the optimized partition was $2.4 \mathrm{~dB}$ over the default partition for $P_{t}=2.5 \mathrm{~W}$. The 


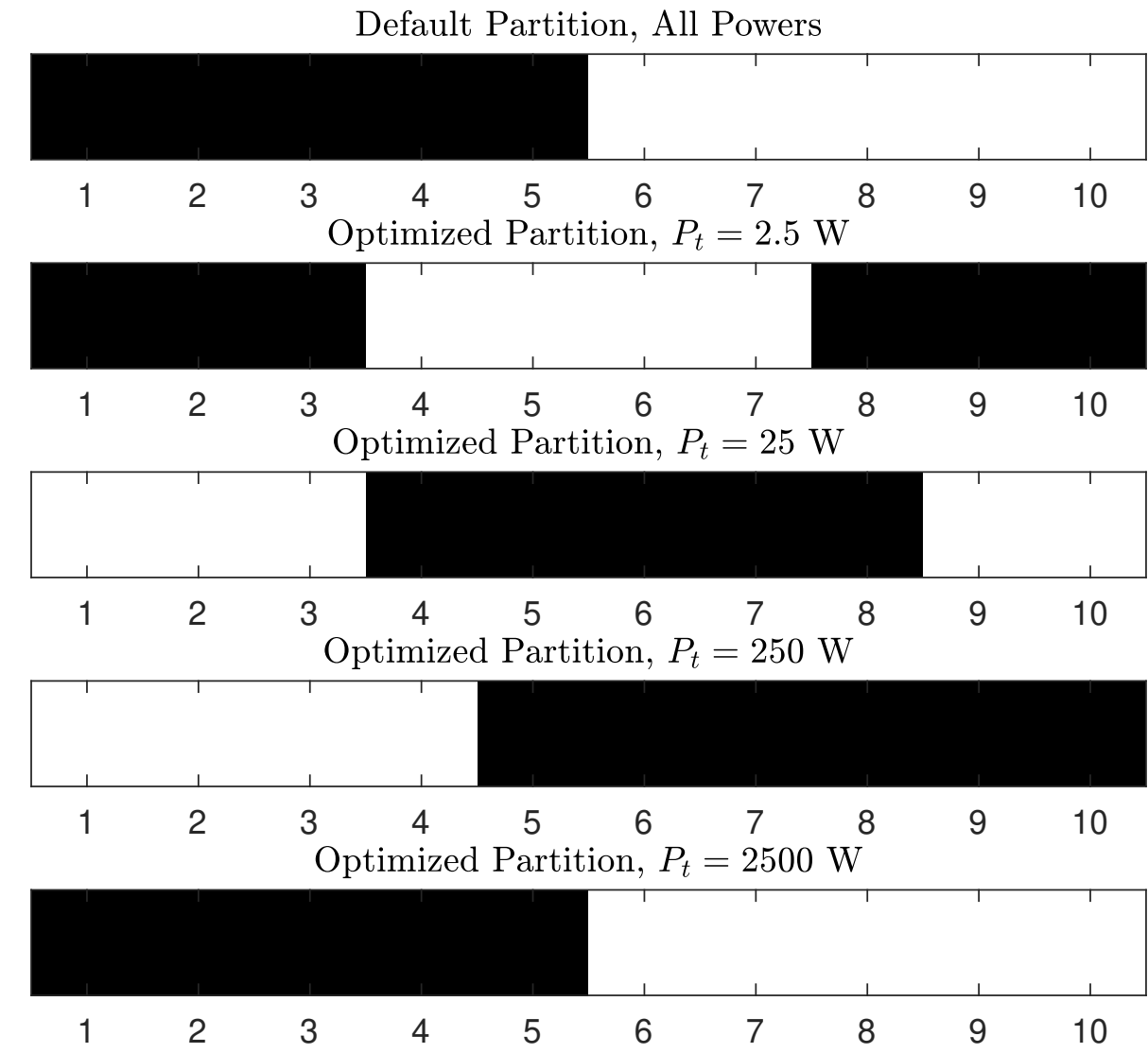

Figure 3.9: Default and optimized transmit/receive aperture partitions for the 10-element array. Black segments represent transmitting antennas and white segments represent receiving antennas. Results are presented for $P_{t}=2.5 \mathrm{~W}, 25 \mathrm{~W}, 250 \mathrm{~W}$, and $2500 \mathrm{~W}$. The GA was run 5 times at each power level. All the trials produced the partition shown below or the equivalent partition with transmit and receive elements swapped.

median gain decreased to effectively zero for $P_{t}=2500 \mathrm{~W}$, as the GA returned the default partition. A small variance was observed in the Bayesian Information reported for the same partitions (relative to the average Bayesian Information at each power level) due to the beamformer optimization technique used in this work. The partition optimization results for the 10-element array suggest that partition optimization is more advantageous for lower power arrays and that beamformer optimization using the default partition may be sufficient for high power arrays. The optimized par- 
titions appear to balance the conflicting goals of maintaining contiguous partitions (which would intuitively make avoiding SI easier for the beamformers) and having transmit and receive apertures that are as wide as possible (which leads to narrower main beams that can better locate targets). As the power level increases, it appears that the incentive to avoid SI dominates the need for narrow main beams.

Searching through 1022 possible solutions for the best partitioning of the aperture may be feasible, but phased arrays often have many more than 10 elements. Because the search space grows exponentially with the number of antennas, it quickly becomes infeasible to exhaust the search space. For example, even with a relatively small $5 \times 10$ element phased array, there are approximately $1.13 \cdot 10^{15}$ possible partitions. Fig. 3.10 shows the gain in Bayesian Information created by partition optimization for a $5 \times 10$-element ALSTAR array. These results suggest that for larger arrays, partition optimization may be more fruitful. It is important to note that elements were added to the array along the $y$-axis (i.e. orthogonal to the azimuth scan plane). Like in the 10-element array case, partition optimization yielded greater improvement in the Bayesian Information metric for the lower transmit power trials. Fig. 3.11 shows the default transmit/receive partition for the $5 \times 10$-element array and the GAoptimized partitions with the highest Bayesian Information across 5 trials for $P_{t}=2.5$ W and 2500 W. As shown in Fig. 3.12 and Fig. 3.13, the partition optimization generally made sacrifices in receiver noise power in order to achieve greater gains in the numerator of the Fisher Information. For $P_{t}=2.5 \mathrm{~W}$, the partition could 


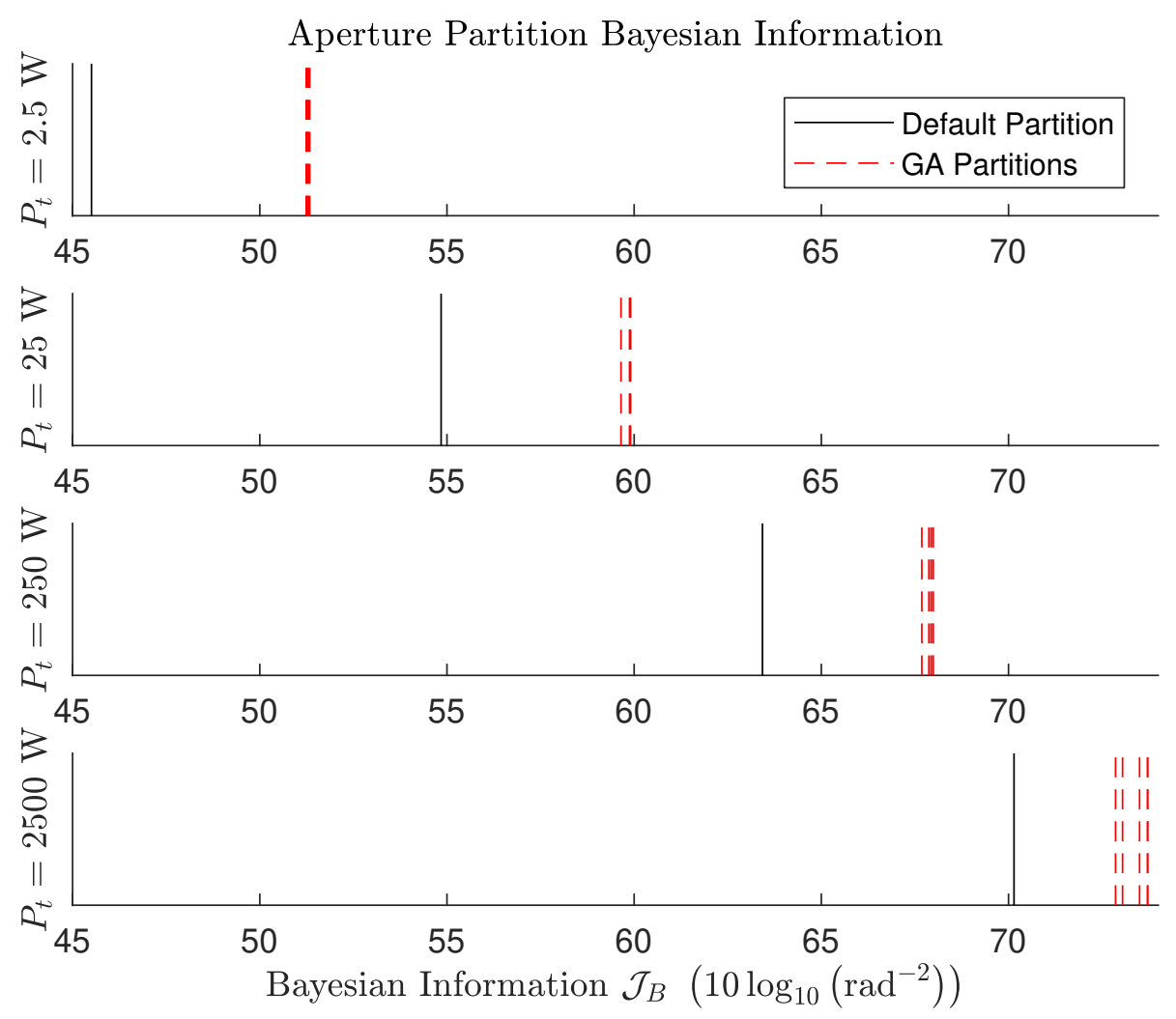

Figure 3.10: Bayesian Information plotted for the default and GA optimized partitions for the $5 \times 10$-element array at $P_{t}=2.5 \mathrm{~W}, 25 \mathrm{~W}, 250 \mathrm{~W}$, and $2500 \mathrm{~W}$.

prioritize improvements in the numerator of the Fisher Information over the small increase in $\sigma_{n}^{2}$ because the low transmit power made noise reduction by beamforming less challenging. Note that for $P_{t}=2500 \mathrm{~W}$, the extra elements in the $y$-axis allow for partitions where the baseline of the transmit and receive apertures are much wider than those in the 10-element array while retaining contiguous partitions that improve inter-aperture isolation. There was more variation in the partitions returned by the GA for the $5 \times 10$-element array trials than seen with the 10 -element array. At the same time, the final performance of these partitions was relatively consistent, suggesting that there may be many partitions that perform well. 

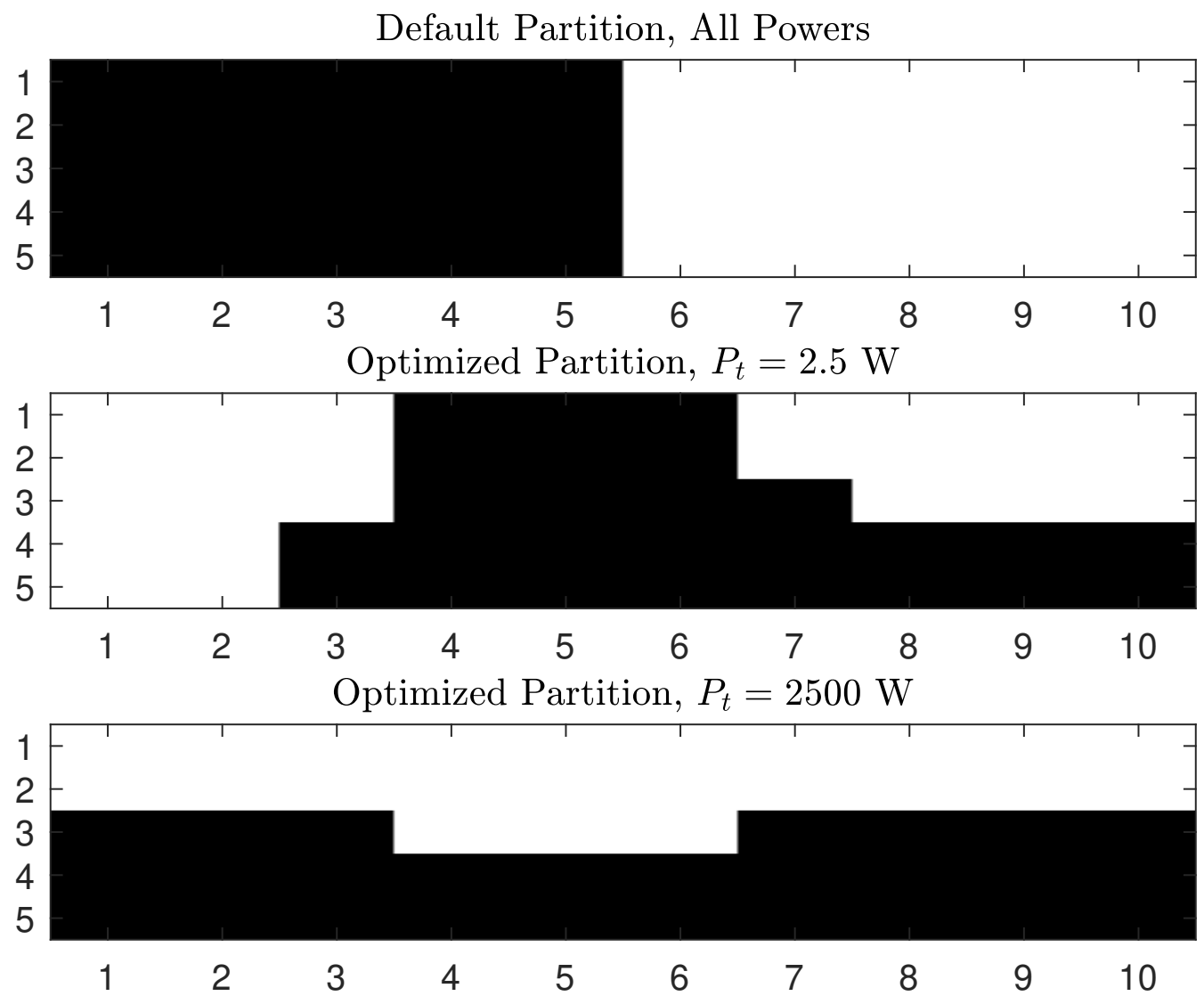

Figure 3.11: Default and Optimized Partitions returned by the GA for the $5 \times 10$-element array. Black segments represent transmitting antennas and white segments represent receiving antennas. The optimized partitions presented here are the best out of 5 GA runs in terms of Bayesian Information.

Fig. 3.14 compares the number of unique function calls required by the GA and exhaustive search for both of the array sizes at each transmit power level. The increase in unique partitions considered by the GA as array size increased was much smaller than the corresponding increase in search space size, suggesting that the GA was effective at reducing the computational effort required to find transmit/receive partitions with significantly improved Bayesian Information. 


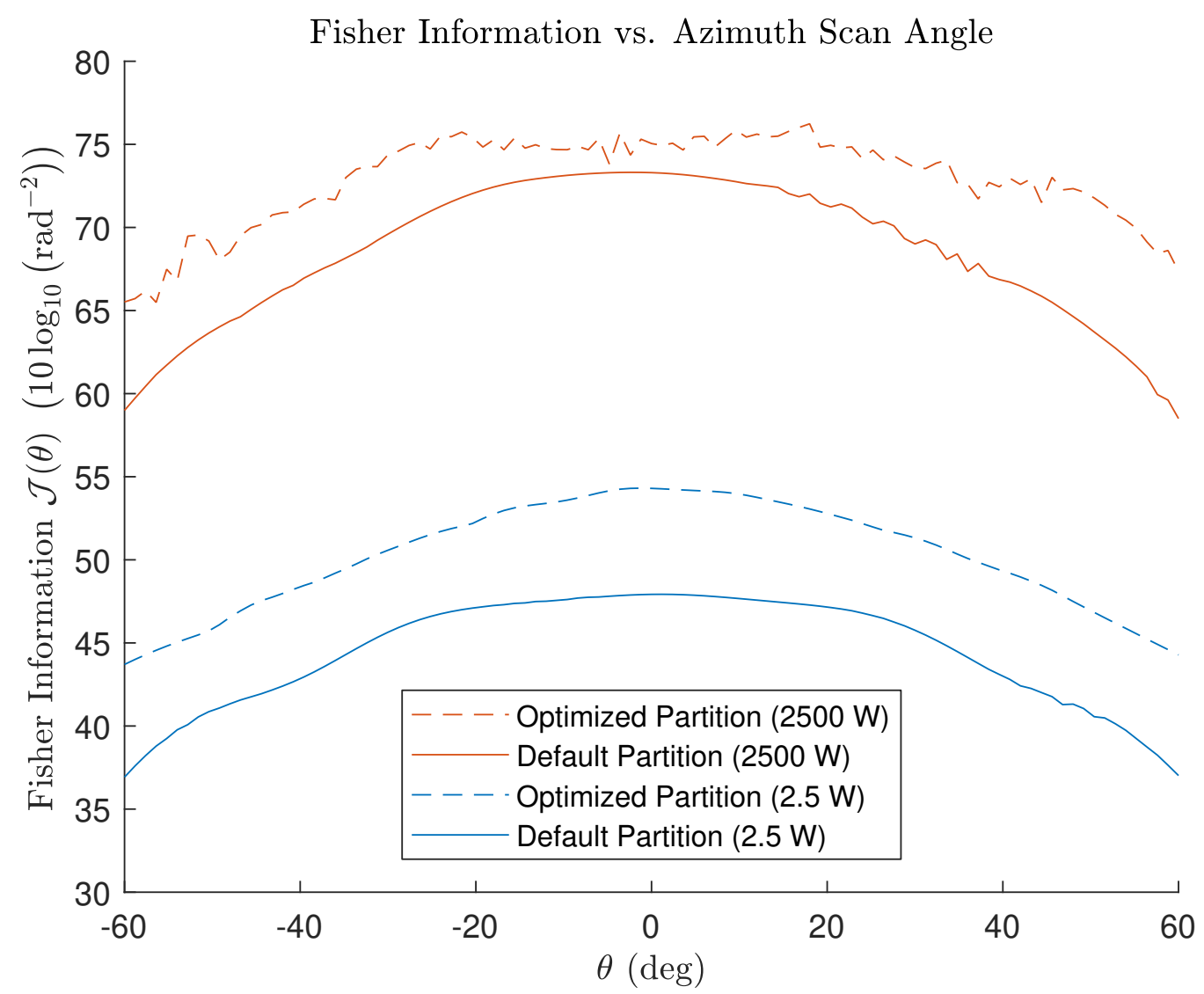

Figure 3.12: Fisher Information $\mathcal{J}(\theta)$ plotted against azimuth scan angle for the default and optimized partitions of the $5 \times 10$-element array at $P_{t}=$ $2.5 \mathrm{~W}$ and $2500 \mathrm{~W}$.

\subsection{Conclusion}

In this work, we set out to explore the narrowband DOA estimation performance of an ALSTAR array that is simultaneously illuminating the scene and measuring the return. We determined the Fisher Information on azimuth-only DOA estimation for ALSTAR arrays and then proposed an adaptive beamforming technique that minimized the Fisher Information of the estimated DOA in terms of the transmit 


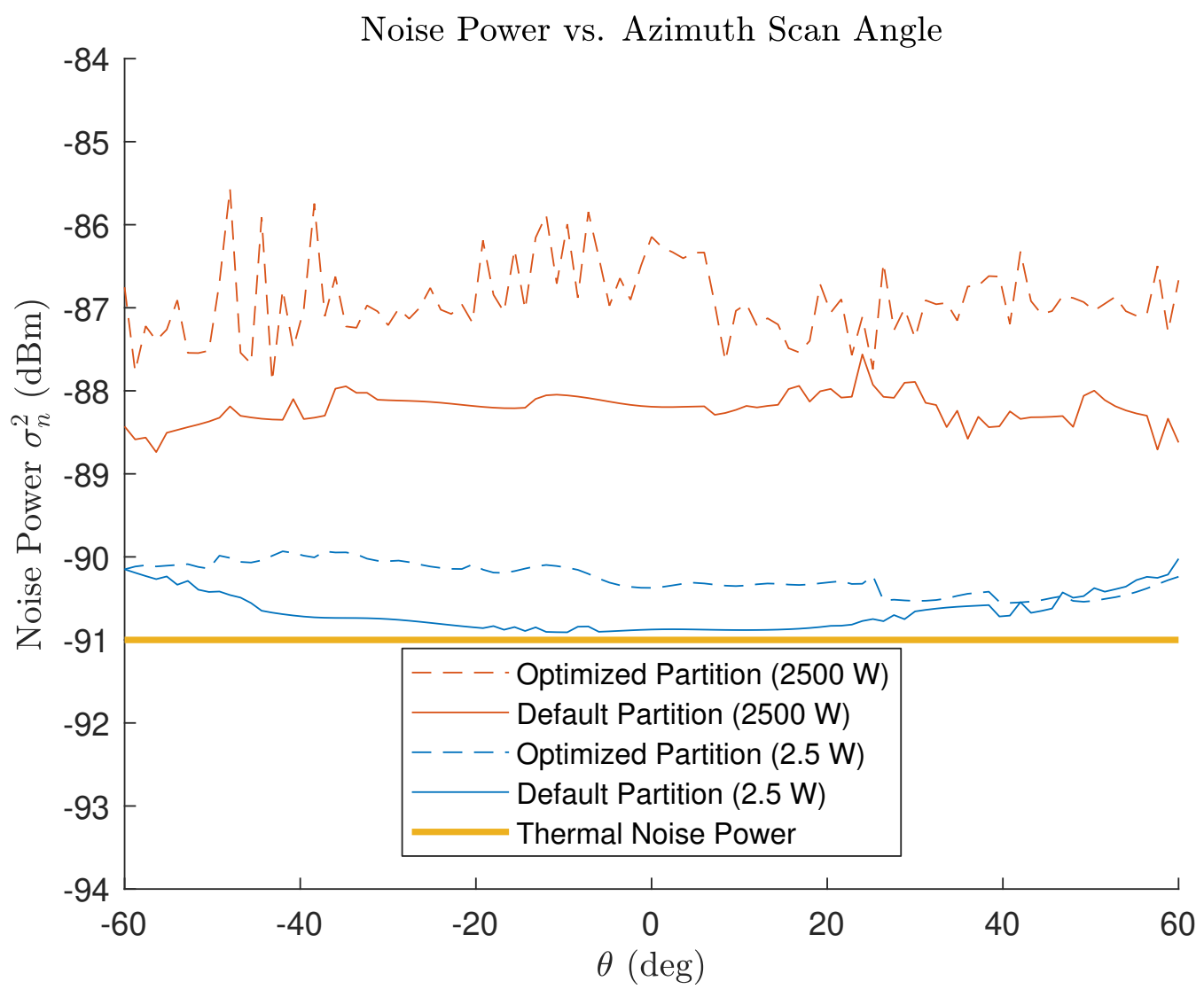

Figure 3.13: Receiver noise power $\sigma_{n}^{2}$ plotted against azimuth scan angle for the default and optimized partitions of the $5 \times 10$-element array at $P_{t}=2.5$ $\mathrm{W}$ and $2500 \mathrm{~W}$.

and receive beamformers. We showed that the adaptive beamformers significantly increased the Fisher Information and thus improved DOA estimation performance. The beamformers returned by the adaptive beamforming algorithm also resulted in high EII and low noise power, which suggests that they are practical for STAR operation. In order to quantify the performance of arrays across the full azimuth scan space, we extended the Fisher Information into the Bayesian Information, which we used as a performance metric when optimizing the transmit/receive partition of the ALSTAR arrays. In order to avoid a computationally intractable exhaustive search 


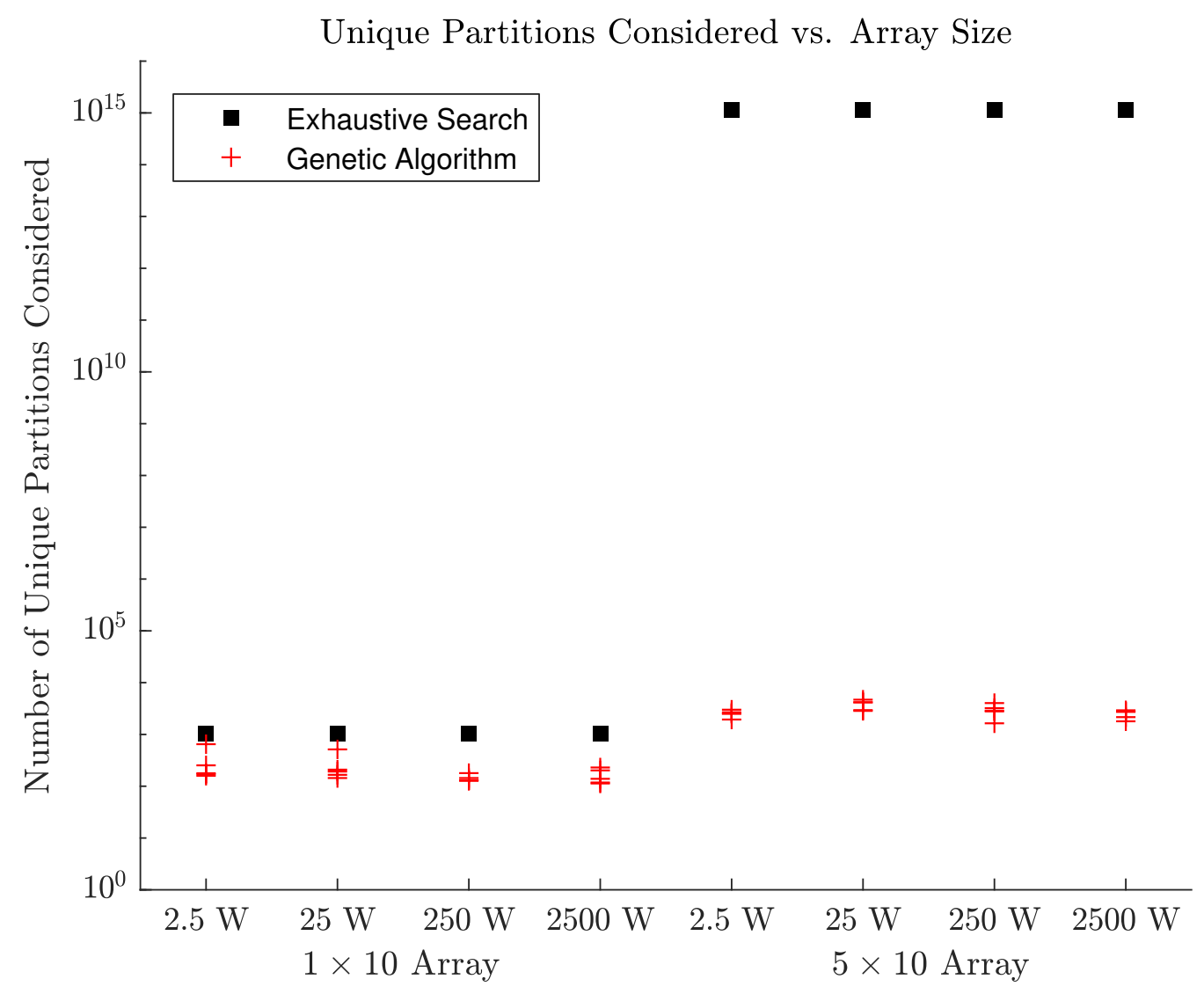

Figure 3.14: Number of unique transmit/receive partitions considered by the GA for both array sizes at each power level across 5 GA trials. The number of partitions that would be considered in an exhaustive search is also plotted for comparison.

among all the possible transmit/receive partitions, we showed that a GA was an effective tool for array optimization. We also showed that under certain conditions, partition optimization (over a default choice of partition) produces worthwhile improvements in the Bayesian Information on the DOA estimate. It is unlikely that the GA described in this work would be suitable for real-time partition optimization on sensor platforms, but it could be useful for optimizing partitions during the array design process. In future work on this topic, it would be valuable to experimentally 
determine the fidelity of the model and predicted performance presented in this paper. Additional work could also seek more computationally efficient methods for the adaptive beamforming and partition optimization tasks presented here, potentially enabling real-time implementations. 
Chapter 4

Adaptive Beamforming and

Mutual Coupling Estimation

Techniques for Aperture-Level

Simultaneous Transmit and

Receive Phased Arrays 


\subsection{Introduction}

In the fields of communication theory and signal processing, it was long assumed that the only means of transmitting and receiving meaningful signals at the same time was to use separate channels in the time, frequency, code, or space domain. This requirement for domain-based separation arose because the massive difference in transmitted and received signal power at a given channel user is so high that the received signal of interest (SoI) would be unresolvable without some effective means of signal separation. Recently, a new paradigm of transmit/receive isolation has developed from the insight that a channel user's knowledge of its own transmitted signals could be used to isolate the SoI from other signals arriving at its receiver. This line of research has been referred to as in-band full-duplex (IBFD) or simultaneous transmit and receive (STAR) and has lead to a variety of solutions. Several recent surveys of the range of proposed solutions are available, specifically [3, 4, 15.

In this work, we focus on achieving STAR in a fully digital phased array of the ALSTAR architecture [1]. In particular, this work compares the performance and computational complexity of several approaches to the adaptive digital beamforming and adaptive digital filtering problems required by the ALSTAR architecture. Specifically, we consider sample matrix inversion (SMI), least mean squares (LMS), and recursive least squares (RLS) channel estimation algorithms. We then describe 
complementary Wiener optimal transmit beamforming and SMI receive beamforming techniques inspired by the narrowband iterative adaptive beamforming proposed in Chapter 2, Finally, we present LMS and RLS alternatives to the SMI adaptive receive beamformer. Using Monte-Carlo trials, we compare the performance of the SMI, LMS, and RLS algorithms for the channel estimation and receive beamforming tasks, respectively. The achievable effective isotropic isolation (EII) and residual interference and noise power are presented for the SMI, LMS, and RLS adaptive beamforming methods while using the SMI channel estimation method. The SMI and RLS adaptive receive beamforming methods achieved $164.6 \mathrm{~dB}$ and $162.8 \mathrm{~dB}$ of average EII at broadside over a $100 \mathrm{MHz}$ bandwidth centered at $2.42 \mathrm{GHz}$. The LMS adaptive receive beamforming method achieves $151.4 \mathrm{~dB}$ of average EII over the same bandwidth, while reducing the computational complexity of the receive beamforming such that it no longer forms the dominant component of the overall computational cost of operating the ALSTAR array. This reduction in computational complexity is the next step in making ALSTAR technology capable of operating in real-time on available hardware. 


\subsubsection{Algorithms for Channel Estimation and Adaptive Beamforming}

The Wiener optimal, SMI, LMS, and RLS adaptive filtering algorithms are well-known and well-established in the field of adaptive signal processing. Haykin studied them all in detail in [167], and presented several example applications. Widrow and McCool studied the differential steepest descent, LMS, and linear random search algorithms [168]. They also described a constrained version of the LMS adaptive beamforming algorithm, but used a different method than the one presented in this work to protect the SoI. Reed et al. compared the convergence performance and complexity of the unconstrained LMS algorithm and the maximum signal to noise (MSN) algorithm to SMI for an adaptive beamforming task [169].

A number of papers have been written about cancelling the self-interference between a single transmit channel and a single receive channel with LMS, RLS, and similar adaptive algorithms. Li et al. simulated a system of digital SIC via the LMS algorithm in a full-duplex (FD) system with a single transmit channel and a single receive channel [170]. Korpi et al. and AghababaeeTafreshi et al. studied nonlinear digital SIC systems controlled by an LMS algorithm for single channel FD radios [171, 172].

Ferrand and Duarte proposed an FD radio with a single transmit antenna and a single receive antenna that used multiple auxiliary receive channels to sample several 
analog delayed versions of the transmitted signal and noise for SIC via a normalized LMS algorithm [173]. Ryu used the LMS algorithm to estimate the residual selfinterference channel after analog self-interference cancellation in an FD radio with one transmit antenna and one receive antenna [174]. Kiayani et al. developed a selfinterference cancellation system that used an auxiliary transmit channel to inject a cancellation signal into the receiver front end before the low-noise amplifier [60]. The digital filters used to create the cancellation signal were controlled by an adaptive LMS-like algorithm. Tamminen et al. demonstrated an LMS-controlled analog SIC system for a single antenna FD radio over several bandwidths up to $80 \mathrm{MHz}$ [175]. Several authors have demonstrated an RF cancellation system controlled by an analog implementation of an LMS algorithm in an FD radio with a single transmit and receive channel [176, 177].

Adams and Bhargava described an RLS channel estimation algorithm for an IBFD system with one transmitting antenna and one receiving antenna [178]. Gebhard et al. presented two versions of the RLS algorithm used for single channel nonlinear interference cancellation in a frequency division duplexed system and demonstrated their performance [179]. Emara et al. proposed an advanced RLS-type algorithm for nonlinear digital SIC in a single antenna FD radio using nonlinear basis functions 180].

Ahmed and Eltawil described an FD system similar to the ALSTAR architecture 
except that it only had one transmit antenna and one receive antenna [181]. They implemented a block-level least-squares self-interference channel estimation protocol during a training interval. Vogt et al. described a self-interference cancellation technique for a single-antenna FD system based on a Kalman filter that accounted for system non-linearities [182]. Enzner described a Bayesian channel estimation algorithm for single time-varying channels built around a simplified Kalman filter [183]. Balatsoukas-Stimming presented a neural network non-linear SIC system for an FD radio with a single transmit antenna and a single receive antenna [184].

Some research has been done on multi-channel adaptive self-interference channel estimation and SIC for antenna arrays. Day et al. described a MIMO adaptive selfinterference channel block-level least squares estimation procedure and associated block-level adaptive beamforming optimization techniques [122]. Masmoudi and LeNgoc developed block-level closed form and iterative maximum likelihood estimators for MIMO SoI and self-interference channels [185]. Anttila et al. implemented nonlinear SIC with linear parameters calculated via a block level least squares procedure in an FD MIMO array [186]. Korpi et al. described a least-squares nonlinear self-interference model coefficient estimation technique for a MIMO array using principle component analysis to reduce the rank of the required pseudo-inversion [187]. Antonio-Rodríguez et al. simulated the performance of an LMS algorithm at estimating the self-interference channel in an FD MIMO relay [188]. Johnston and Fiore proposed an LMS channel estimation technique for the mutual coupling channel in 
an FD antenna array [77]. The LMS channel estimation technique presented in this work was based on Johnston and Fiore's presentation of the method. Lemos et al. described a mutual coupling channel estimation technique based on the RLS algorithm which they used for SIC in a MIMO decode and forward relay [189].

To our knowledge, this paper is the first work where adaptive digital SIC and adaptive digital beamforming controlled by the LMS or RLS algorithm have been applied in concert to the problem of achieving IBFD or STAR in a fully-digital phased array.

\subsubsection{Our Previous Work}

The ALSTAR architecture that forms the basis of this work was originally presented in [1]. An 8-element ALSTAR array was built and evaluated in [6]. Along with Chapter 3, several of our previous papers discussed the performance bounds of the ALSTAR architecture in narrowband imaging/direction-finding tasks and how the beamforming and transmit/receive partitioning of the aperture could be optimized to minimize those bounds [127, 128]. We also explored optimizing the mean squared error (MSE) bound on SoI phase estimation in ALSTAR phased arrays in an FD narrowband communications scenario with regard to the beamformers and aperture partitions [129]. In [190], we showed that it was possible to perform approximate narrowband adaptive beamforming for a 10-element ALSTAR array with a shallow 
neural network. The work presented here extends Chapter 2, which set out the achievable EII for a narrowband ALSTAR array and highlighted the interdependence of the transmit and receive beamformers in maximizing that isolation. That work assumed that the exact mutual coupling matrix was known. This work assumes that the adaptive beamforming and self-interference cancellation methods only have access to the observable signals in the array model and the direction of arrival of the SoI. Additionally, this work also accounts for the possible presence of external interference in the adaptive beamforming process, whereas Chapter 2 did not.

\subsubsection{Outline}

In Section 4.2 we describe the ALSTAR digital phased array architecture and the corresponding signal model in detail. In Section 4.3 we outline the implementations of the SMI, LMS, and RLS channel estimation algorithms used in this work, present simulated results, and discuss the relative computational complexity of the algorithms. Section 4.4 shows how the beamforming concepts presented in Chapter 2 can be extended to operate on a realistic wideband signal model. It also discusses the LMS and RLS adaptive receive beamforming methods as alternatives to SMI, and presents the simulated performance of the adaptive algorithms in terms of isolation and computational efficiency. Finally, Section 4.5 draws conclusions from the simulation results and discusses potential avenues of future research. 


\subsubsection{Notation}

In this work, we use the following notational conventions. Scalar variables are denoted with lowercase letters, vector variables with bold lowercase letters, and matrix variables with bold uppercase letters. $(\cdot)^{*}$ indicates conjugation, $(\cdot)^{T}$ indicates transposition, and $(\cdot)^{\mathrm{H}}$ indicates Hermitian transposition. The Diag $(\cdot)$ operator accepts a vector argument and places its entries on the diagonal of a square matrix with the same dimension. Convolution is indicated by the symbol $*$ and circular convolution is indicated by the symbol $\circledast . E\{\cdot\}$ denotes the expectation operator, $\mathcal{F}^{-1}\{\cdot\}$ denotes the inverse Fast Fourier Transform, and $\mathcal{O}(\cdot)$ indicates the "big-Oh" computational complexity of an algorithm. Parenthesized superscripts denote channel indices. Indices appearing to the right of a variable in square brackets indicate a time index. In the case of quantities with a sample index, if no index is provided, the symbol refers to all of the samples in a row vector or matrix (i.e. $a=[a[1], \cdots, a[n]]$ or $\mathbf{a}=[\mathbf{a}[1], \cdots, \mathbf{a}[n]])$. Indices appearing to the right of a variable in parentheses indicate a specific element or range of elements in the corresponding vector or matrix variable. For convenience, we define the operator $\mathrm{C}^{N}(\cdot)$ which forms a convolution matrix from its argument by repeating each of its rows $N$ times after adding incremental zero-padding to the beginning of each repeated row. We also define the operator $\tilde{\mathrm{C}}^{N}(\cdot)$ which forms a circular convolution matrix from its argument by repeating each of its rows $N$ times with a circular shift for each repetition of a given row. 


\subsection{ALSTAR Array Signal Model}

The ALSTAR phased array architecture consists of $T+R$ antennas that can be dynamically configured to transmit or receive, where $T$ antennas have been configured to transmit and $R$ antennas have been configured to receive. Fig. 4.1(a) depicts the ALSTAR architecture and Fig. 4.1(b) defines the symbols used to describe the signals, propagation path models, and filters involved in the architecture. While the ALSTAR architecture was originally proposed in [1, an extension of that signal model to account for limited dynamic range transmit and receive channels was presented in Chapter 2. We further extend the signal model here to account for external sources of interference and to account for imperfect mutual coupling channel estimation. The ALSTAR architecture is faced with the task of transmitting a signal $x_{t}[n] \in \mathbb{C}$ at time index $n$ in a given direction while simultaneously receiving an SoI, whose components at each receive antenna are represented by $s^{(k)}[n] \in \mathbb{C}$. We assume that the signals involved are sampled at baseband frequency $f_{s}$. The signal transmitted by the $j$ th antenna at time $n$ is given by

$$
t^{(j)}[n]=\left(\mathbf{h}_{t}^{(j)} * x_{t}\right)[n]+n_{t}^{(j)}[n], \quad 1 \leq j \leq T,
$$




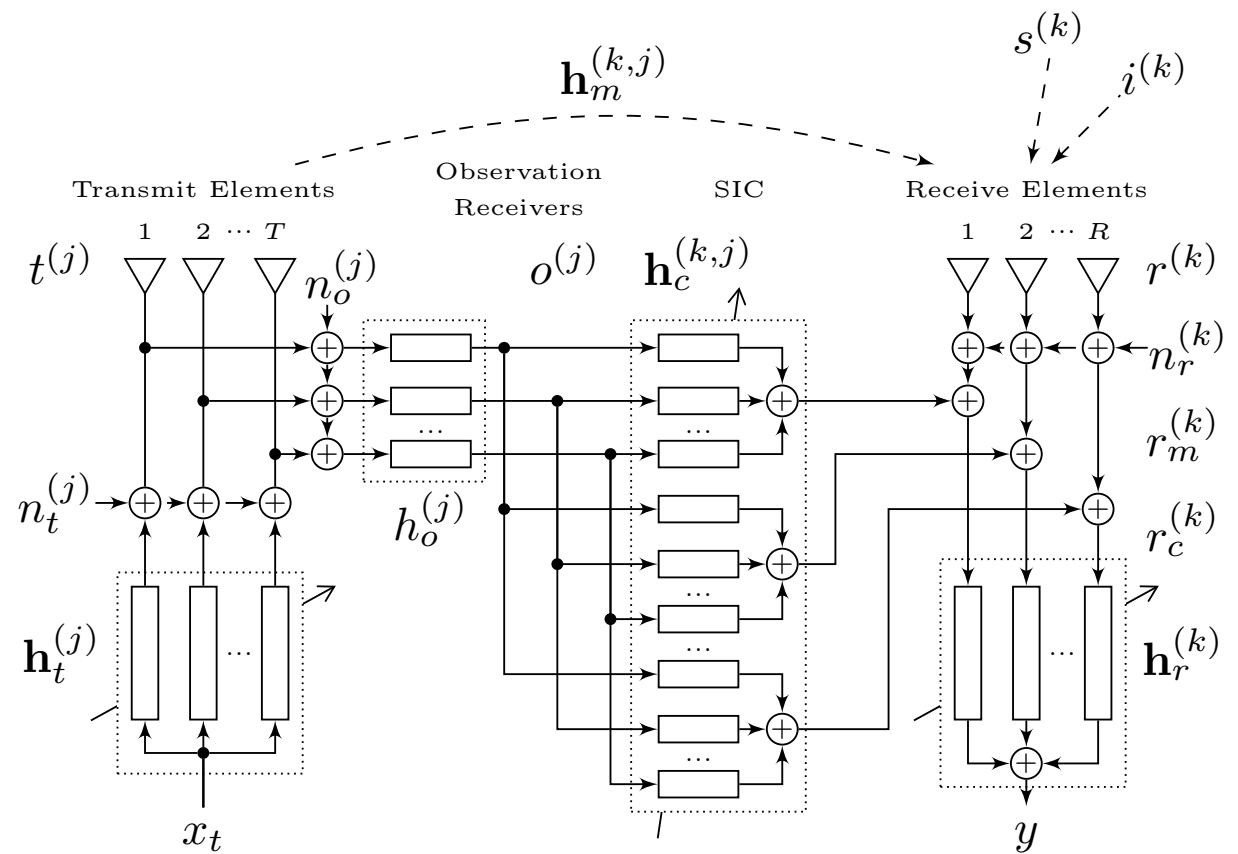

(a)

\begin{tabular}{cl} 
Symbol & Definition \\
\hline$x_{t}$ & Transmitted Signal \\
$\mathbf{h}_{t}^{(j)}$ & $j$ th Transmit Channel Filter \\
$n_{t}^{(j)}$ & $j$ th Transmit Channel Noise \\
$t^{(j)}$ & $j$ th Transmit Channel Signal + Noise \\
$n_{o}^{(j)}$ & $j$ th Observation Channel Noise \\
$h_{o}^{(j)}$ & $j$ th Observation Channel Attenuation \\
$o^{(j)}$ & $j$ th Observation Channel Signal \\
$\mathbf{h}_{c}^{(k, j)}$ & $(k, j)$ th SIC Filter \\
$\mathbf{h}_{m}^{(k, j)}$ & $(k, j)$ th Mutual Coupling Channel \\
$s^{(k)}$ & $k$ th Receive Channel SoI \\
$i^{(k)}$ & $k$ th Receive Channel External Interference \\
$r^{(k)}$ & Signal at $k$ th Receive Antenna \\
$n_{r}^{(k)}$ & $k$ th Receive Channel Noise \\
$r_{m}^{(k)}$ & Signal Measured at $k$ th Receive Channel ADC \\
$r_{c}^{(k)}$ & $k$ th Receive Channel Signal after SIC \\
$\mathbf{h}_{r}^{(k)}$ & $k$ th Receive Channel Filter \\
$y$ & Received Beamformed Signal \\
\hline
\end{tabular}

(b)

Figure 4.1: (a) ALSTAR digital phased array architecture signal flow diagram, including digital SIC, (b) Symbols and corresponding definitions for the ALSTAR architecture, modified from [1] and Chapter 2. 
where $\mathbf{h}_{t}^{(j)} \in \mathbb{C}^{N_{t} \times 1}$ is the $N_{t}$-tap finite impulse response (FIR) filter for the $j$ th transmit channel and

$$
n_{t}^{(j)}[n] \sim \mathcal{C N}\left(0, \eta_{t}^{-1}\left\|\mathbf{h}_{t}^{(j)}\right\|^{2}\right)
$$

is independent additive white Gaussian noise (AWGN) that models the limited transmit channel dynamic range $\eta_{t}$. The signals incident on the $k$ th receive antenna at time $n$ are modeled by

$$
\begin{aligned}
r^{(k)}[n]= & s^{(k)}[n]+i^{(k)}[n] \\
& +\sum_{j=1}^{T}\left(\mathbf{h}_{m}^{(k, j)} * t^{(j)}\right)[n], \quad 1 \leq k \leq R .
\end{aligned}
$$

Here, $i^{(k)}[n] \in \mathbb{C}$ represents the external interference signal at each antenna, and the last term describes the self-interference created by the mutual coupling between the transmit and receive antennas, where $\mathbf{h}_{m}^{(k, j)} \in \mathbb{C}^{N_{m} \times 1}$ describes the $N_{m}$-tap mutual coupling channel from the $j$ th transmit antenna to the $k$ th receive antenna. We notate the received signals and noise measured at the ADCs by

$$
r_{m}^{(k)}[n]=r^{(k)}[n]+n_{r}^{(k)}[n], \quad 1 \leq k \leq R
$$

where

$$
n_{r}^{(k)}[n] \sim \mathcal{C N}\left(0, \eta_{r}^{-1} E\left\{\left|r^{(k)}[n]\right|^{2}\right\}+\sigma_{r}^{2}\right)
$$


is independent AWGN noise that models the limited dynamic range of the receive channels $\eta_{r}$, which also have a fixed thermal noise power component $\sigma_{r}^{2}$.

\subsubsection{Digital SIC in ALSTAR}

In order to achieve STAR, the ALSTAR architecture must sufficiently cancel or suppress the interference and noise components in 4.4. In many STAR scenarios, the self-interference component is the strongest, and is mitigated in part via SIC. The ALSTAR architecture implements SIC with observed copies of the transmitted signal, noise, and distortion from each transmit channel. In an ALSTAR phased array, each antenna can be configured to transmit or receive, as each antenna is connected to independent transmit and receive hardware via a switching system, as shown in Fig. 4.2. When the antenna is configured to receive, the transmit hardware is disconnected and the receive hardware is connected to the antenna via a low-noise amplifier. When the antenna is configured to transmit, the transmit RF hardware is connected directly to the antenna and the receive $\mathrm{RF}$ hardware is connected to a reference tap near the antenna through a fixed attenuator. In this way, the transmitted signal and noise are observed via the receive hardware behind the transmitting antennas. We model the observed signals as

$$
o^{(j)}[n]=h_{o}^{(j)}\left(t^{(j)}[n]+n_{o}^{(j)}[n]\right), \quad 1 \leq j \leq T,
$$




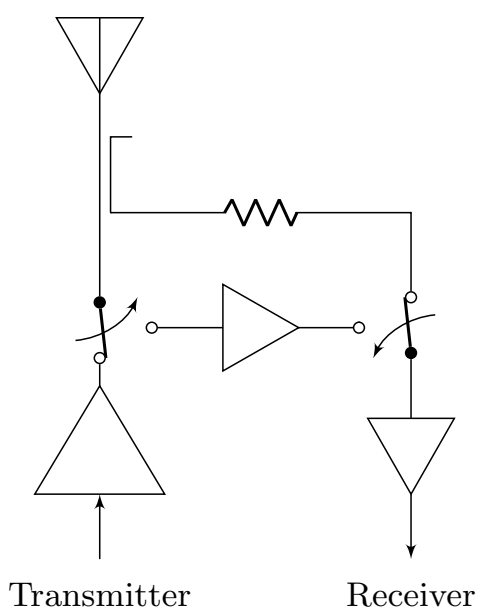

Figure 4.2: Schematic of the switching system that enables each antenna to transmit or receive. When configured to transmit, the antenna is connected to the transmit RF front end via a power amplifier, and the transmitted signal, noise, and distortion are measured by the otherwise unused receive hardware. The receive channel is coupled to the reference tap on the antenna through a fixed attenuator in order to prevent receiver saturation or damage. When configured to receive (arrows), the receiver is coupled to the antenna through a low-noise amplifier, and the transmit channel is disconnected [1].

where $h_{o}^{(j)}$ represents the fixed attenuation in the observation channels to prevent receiver saturation and

$$
n_{o}^{(j)}[n] \sim \mathcal{C N}\left(0, \eta_{r}^{-1}\left\|\mathbf{h}_{t}^{(j)}\right\|^{2}\right)
$$

is independent AWGN noise that models the limited dynamic range of the observation receivers. In an actual system, the observation channels $h_{o}^{(j)}, 1 \leq j \leq T$ would be better modeled by multi-tap FIR filters and would need to be estimated. The observation filter estimation error could degrade the mutual coupling channel estimation and SIC performance if the filter estimate was not sufficiently accurate. Because the increased mathematical complexity of handling multi-tap observation channel models 
would not contribute to demonstrating the performance of the adaptive beamforming and digital SIC methods in the ALSTAR architecture, and because reasonably accurate channel estimation methods for channels like this are readily available, we believe that our assumption of single-tap channels with known weights is justified for the purposes of this work.

In order to cancel the self-interference from the received signal, the ALSTAR architecture passes the observed signals, noise, and distortion through a bank of SIC filters $\mathbf{h}_{c}^{(k, j)} \in \mathbb{C}^{N_{m} \times 1}, 1 \leq k \leq R, 1 \leq j \leq T$, and adds the result to the received signals $r_{m}^{(k)}$ to form the received signals after SIC,

$$
\begin{aligned}
r_{c}^{(k)}[n]= & s^{(k)}[n]+i^{(k)}[n]+\sum_{j=1}^{T}\left(\mathbf{h}_{m}^{(k, j)} * t^{(j)}\right)[n]+n_{r}^{(k)}[n] \\
& +\sum_{j=1}^{T}\left(\mathbf{h}_{c}^{(k, j)} * h_{o}^{(j)}\left(t^{(j)}+n_{o}^{(j)}\right)\right)[n] .
\end{aligned}
$$

Given perfect knowledge of $\mathbf{h}_{m}^{(k, j)}, 1 \leq k \leq R, 1 \leq j \leq T$, we could set

$$
\mathbf{h}_{c}^{(k, j)}=-\left(h_{o}^{(j)}\right)^{-1} \mathbf{h}_{m}^{(k, j)},
$$


and the isolated received signal would become

$$
\begin{aligned}
y[n]=\sum_{k=1}^{R}\left(\mathbf { h } _ { r } ^ { ( k ) * } * \left(s^{(k)}+i^{(k)}\right.\right. & \\
& \left.\left.\quad-\sum_{j=1}^{T}\left(\mathbf{h}_{m}^{(k, j)} * n_{o}^{(j)}\right)+n_{r}^{(k)}\right)\right)[n] .
\end{aligned}
$$

Thus, digital SIC using observed signals and knowledge of the mutual coupling channel can eliminate the signal, noise, and distortion components of the self-interference at the cost of introducing a virtually-coupled observation noise term (i.e. the first term on the second line in (4.10)). This additional term will generally have lower power than the mutually-coupled noise and distortion because the observation channel dynamic range is generally much larger than the transmit channel dynamic range-i.e., $\eta_{r} \gg \eta_{t}$ - for typical transmit and receive hardware.

In practice, the mutual coupling channel must be estimated. The estimate of the mutual coupling channel $\hat{\mathbf{h}}_{m}^{(k, j)}$ will not be exact. Setting

$$
\mathbf{h}_{c}^{(k, j)}=-\left(h_{o}^{(j)}\right)^{-1} \hat{\mathbf{h}}_{m}^{(k, j)}
$$

implies

$$
y[n]=\sum_{k=1}^{R}\left(\mathbf{h}_{r}^{(k) *} * r_{c}^{(k)}\right)[n],
$$


where

$$
\begin{aligned}
r_{c}^{(k)}[n]= & s^{(k)}[n]+i^{(k)}[n] \\
& +\sum_{j=1}^{T}\left(\left(\mathbf{h}_{m}^{(k, j)}-\hat{\mathbf{h}}_{m}^{(k, j)}\right) * t^{(j)}\right)[n] \\
& -\left(\hat{\mathbf{h}}_{m}^{(k, j)} * n_{o}^{(j)}\right)[n]+n_{r}^{(k)}[n] .
\end{aligned}
$$

These equations highlight the importance of obtaining an accurate estimate of $\mathbf{h}_{m}^{(k, j)}$. Practically speaking, the performance of the system will approach the performance of a system with perfect channel knowledge when the power of the residual selfinterference signal $\left(\mathbf{h}_{m}^{(k, j)}-\hat{\mathbf{h}}_{m}^{(k, j)}\right) * t^{(j)}$ falls below the power of the other interference and noise terms.

\subsubsection{Effective Isotropic Isolation}

The definition of EII was originally given in [1] and that definition was extended in Chapter 2 to account for limited dynamic range transmit and receive channels under the assumption that the mutual coupling channel was known a priori and that there was no external interference. In this work we do not make these assumptions. EII is defined as the ratio of effective isotropic radiated power (EIRP) to effective isotropic sensitivity (EIS),

$$
\mathrm{EII}=\frac{\mathrm{EIRP}}{\mathrm{EIS}}
$$


It will sometimes be convenient to refer to the stacked channel FIR filters as the transmit beamformer

$$
\mathbf{b}_{t}=\left[\left(\mathbf{h}_{t}^{(1)}\right)^{T} \cdots\left(\mathbf{h}_{t}^{(j)}\right)^{T} \cdots\left(\mathbf{h}_{t}^{(T)}\right)^{T}\right]^{T}
$$

and receive beamformer

$$
\mathbf{b}_{r}=\left[\left(\mathbf{h}_{r}^{(1)}\right)^{T} \cdots\left(\mathbf{h}_{r}^{(k)}\right)^{T} \cdots\left(\mathbf{h}_{r}^{(R)}\right)^{T}\right]^{T},
$$

respectively.

EIRP is the power that an isotropic antenna would have to radiate to match the power of the main transmit beam of the array, and is calculated by

$$
\operatorname{EIRP}(\phi, \theta)=g(\theta) \mathbf{b}_{t}^{\mathrm{H}} \mathbf{q}_{t}(\phi, \theta) \mathbf{q}_{t}(\phi, \theta)^{\mathrm{H}} \mathbf{b}_{t},
$$

where $\phi$ is the direction of the main beam in the plane of the array measured from the $+x$-axis and $\theta$ is the angle between the main beam and broadside. We model the embedded antenna element gain as $g(\theta)=\pi \cos \theta$. The transmit array manifold vector is

$$
\mathbf{q}_{t}(\phi, \theta)=\left[\left(\mathbf{q}_{t}^{(1)}\right)^{T} \cdots\left(\mathbf{q}_{t}^{(j)}\right)^{T} \cdots\left(\mathbf{q}_{t}^{(T)}\right)^{T}\right]^{T}
$$


where

$$
\mathbf{q}_{t}^{(j)}=\mathcal{F}^{-1}\left\{\frac{e^{-j 2 \pi \mathbf{f}_{t}\left(\frac{x_{t}(j) \sin \theta \cos \phi+y_{t}(j) \sin \theta \sin \phi}{c}+\frac{N_{t}-1}{2 f_{s}}\right)}}{N_{t}}\right\}
$$

Also, the frequency vector $\mathbf{f}_{t}$ is defined as

$$
\mathbf{f}_{t}=\left[-\frac{f_{s}}{2},-\frac{f_{s}}{2}+\frac{f_{s}}{N_{t}-1}, \cdots, \frac{f_{s}}{2}\right]^{T} .
$$

Notice that $\mathbf{q}_{t}^{(j)}$ is simply a fractional delay filter that creates the appropriate delay in the transmitted signal to form a beam in the desired direction. The last term in the exponent centers the zero-delay point in the middle of the filter to avoid edge effects.

EIS is the sensitivity that would be required of an isotropic antenna to match the sensitivity of the receive array in the direction of the receive beam. Mathematically it can be expressed as the ratio of interference and noise power in the isolated received signal to the receive gain,

$$
\mathrm{EIS}=\frac{P_{i+n}}{G_{r}(\phi, \theta)}
$$

Receive gain is calculated by

$$
G_{r}(\phi, \theta)=g(\theta) \mathbf{b}_{r}^{\mathrm{H}} \mathbf{q}_{r}(\phi, \theta) \mathbf{q}_{r}(\phi, \theta)^{\mathrm{H}} \mathbf{b}_{r} .
$$


The receive array manifold vector is

$$
\mathbf{q}_{r}(\phi, \theta)=\left[\left(\mathbf{q}_{r}^{(1)}\right)^{T} \cdots\left(\mathbf{q}_{r}^{(k)}\right)^{T} \cdots\left(\mathbf{q}_{r}^{(R)}\right)^{T}\right]^{T}
$$

where

$$
\mathbf{q}_{r}^{(k)}=\mathcal{F}^{-1}\left\{\frac{e^{j 2 \pi \mathbf{f}_{r}\left(\frac{x_{r}(k) \sin \theta \cos \phi+y_{r}(k) \sin \theta \sin \phi}{c}+\frac{N_{r}-1}{2 f_{s}}\right)}}{N_{r}}\right\}
$$

The frequency vector $\mathbf{f}_{r}$ is defined similarly to $\mathbf{f}_{t}$ except that $N_{t}$ is replaced with $N_{r}$. The sign of the exponent in the receive array manifold vector is opposite that of the transmit manifold in order to account for the fact that the receive filters are applied to the received signals after conjugation. This matches the conventions in our previous work at narrowband in Chapter 2. The interference and noise power in the isolated received signal (i.e., after $\mathrm{SIC}$ ) is

$$
P_{i+n}=E\left\{\left|y[n]-\sum_{k=1}^{R}\left(\mathbf{h}_{r}^{(k) *} * s^{(k)}\right)[n]\right|^{2}\right\} .
$$

In order to maximize EII and achieve STAR functionality, the ALSTAR architecture is faced with two major tasks: mutual coupling channel estimation and adaptive beamforming. The adaptive beamforming and SIC methods proposed previously in Chapter 2 for the ALSTAR architecture made several strong simplifying assumptions. First, that work assumed perfect knowledge of the coupling channel. Second, the presence of the SoI and external interference was ignored in the discussion of adaptive 
beamforming. The channel estimation and adaptive beamforming techniques presented in this work do not make any of those assumptions, and thus represent a much more practical and realistic approach. The next section will compare the SMI, LMS, and RLS channel estimation techniques in terms of their computational cost and accuracy in the ALSTAR architecture.

\subsection{Mutual Coupling Channel Estimation}

As discussed in the previous section, an accurate estimate of the mutual coupling channel is critical for high EII. In this section we will compare the performance of the SMI, LMS, and RLS algorithms for the mutual coupling channel estimation task in the context of the ALSTAR architecture. For all the algorithms, mutual coupling channel probing is done prior to the first channel access. Each transmit channel is excited independently in sequence in order to improve the convergence performance and final accuracy of the channel estimates.

\subsubsection{SMI Channel Estimation}

In estimating the mutual coupling channel, the channel output of interest is $\mathbf{r}_{m}[n]$ and the channel input is $t^{(j)}[n]$. Because we do not have direct access to $t^{(j)}[n]$, we must 
use the measured transmitted signal and noise, given by $\left(h_{o}^{(j)}\right)^{-1} o^{(j)}[n]$. It is helpful here to stack the individual estimated channels corresponding to the $j$ th transmit channel into a single channel matrix,

$$
\widehat{\mathbf{M}}_{r}^{(j)}=\left[\begin{array}{c}
\left(\hat{\mathbf{h}}_{m}^{(1, j)}\right)^{T} \\
\vdots \\
\left(\hat{\mathbf{h}}_{m}^{(R, j)}\right)^{T}
\end{array}\right] .
$$

If we form the time correlation matrix of the channel input over $S$ samples,

$$
\mathbf{R}_{o o}^{(j)}=\left(\left(h_{o}^{(j)}\right)^{2} \cdot S\right)^{-1} \mathrm{C}^{N_{m}}\left(o^{(j)}\right) \cdot \mathrm{C}^{N_{m}}\left(o^{(j)}\right)^{\mathrm{H}},
$$

and the cross-correlation matrix between the channel input and receive channel taps,

$$
\mathbf{R}_{r o}^{(j)}=\left(h_{o}^{(j)} \cdot S\right)^{-1} \mathbf{r}_{m} \cdot \mathrm{C}^{N_{m}}\left(o^{(j)}\right)^{\mathrm{H}}
$$

then we can estimate the portion of the mutual coupling channel corresponding to the $j$ th transmit channel as

$$
\widehat{\mathbf{M}}_{r}^{(j)}=\mathbf{R}_{r o}^{(j)}\left(\mathbf{R}_{o o}^{(j)}\right)^{-1} .
$$




\subsubsection{LMS Channel Estimation}

The LMS algorithm used in this work was based on the presentations found in [77] and [167]. The key insight behind the LMS algorithm for channel estimation is to compare the measured and predicted outputs of a channel given some known input. The product of the channel input and the conjugated error between the predicted and actual channel outputs forms the update direction of the adaptive method. The update equation for the channel matrix is

$$
\widehat{\mathbf{M}}_{r}^{(j)}[n+1]=\widehat{\mathbf{M}}_{r}^{(j)}[n]+\frac{\mu_{m}}{\left\|\mathbf{u}_{o}[n]\right\|^{2}} \boldsymbol{\Delta}_{m}[n] \cdot \mathbf{u}_{o}^{\mathrm{H}}[n]
$$

where $n$ denotes the time index of the estimate within the current block,

$$
\boldsymbol{\Delta}_{m}[n]=\mathbf{r}[n]-\widehat{\mathbf{M}}_{r}^{(j)}[n] \cdot \mathbf{u}_{o}[n]
$$

is the error between the measured and predicted channel outputs, and

$$
\mathbf{u}_{o}[n]=\left(h_{o}^{(j)}\right)^{-1}\left[o^{(j)}[n] \cdots o^{(j)}\left[n-N_{m}+1\right]\right]^{T}
$$

is the measured channel input. The parameter $\mu_{m}$ tunes the learning rate, and can be chosen on the interval $\mu_{m} \in(-\infty, 3) \mathrm{dB}$, (i.e. $\mu_{m} \in(0,2)$ ). 


\subsubsection{RLS Channel Estimation}

The RLS implementation used in this work is based on [167]. The structure of the RLS channel estimation technique is similar to the LMS technique, except that the RLS algorithm maintains an estimate of the inverse input correlation matrix $\mathbf{P}_{m}$ and uses it to calculate a whitened version of the channel inputs

$$
\mathbf{c}_{m}[n]=\lambda_{m}^{-1} \mathbf{P}_{m}[n] \mathbf{u}_{o}[n]
$$

where $\mathbf{P}_{m}[0]=\delta_{m}^{-1} \mathbf{I}_{N_{m}}$ with regularization parameter $\delta_{m}$. The parameter $\lambda_{m} \in(0,1]$ tunes the aggressiveness of the updates for the RLS algorithm. It is convenient to define a scaled version of the whitened input vector,

$$
\mathbf{k}_{m}[n]=\left(1+\mathbf{u}_{o}^{\mathrm{H}}[n] \mathbf{c}_{m}[n]\right)^{-1} \mathbf{c}_{m}[n]
$$

Now we can write the update equation for the channel model in the RLS algorithm,

$$
\widehat{\mathbf{M}}_{r}^{(j)}[n+1]=\widehat{\mathbf{M}}_{r}^{(j)}[n]+\boldsymbol{\Delta}_{m}[n] \mathbf{k}_{m}^{\mathbf{H}}[n]
$$

Finally, we must also update the estimated inverse correlation matrix

$$
\mathbf{P}_{m}[n+1]=\lambda_{m}^{-1}\left(\mathbf{P}_{m}[n]-\mathbf{k}_{m}[n] \mathbf{u}_{o}^{\mathrm{H}}[n] \mathbf{P}_{m}[n]\right)
$$




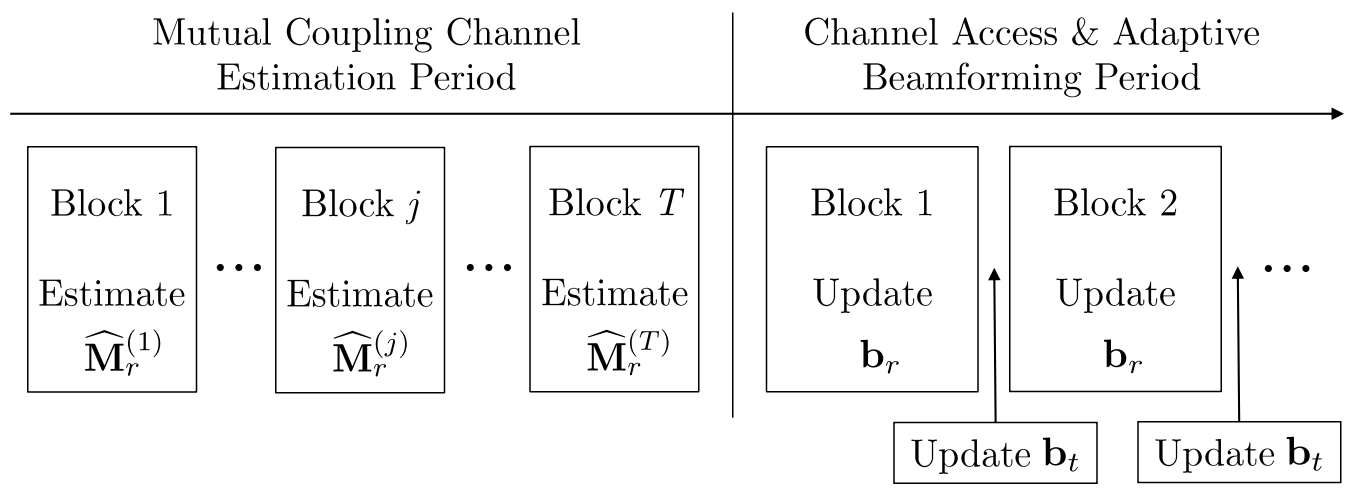

Figure 4.3: Outline of the operation of the channel estimation and adaptive beamforming techniques proposed in this work.

\subsubsection{Performance Comparison Results}

In order to determine the optimal values of $\mu_{m}$ for the LMS algorithm as well as $\lambda_{m}$ and $\delta_{m}^{-1}$ for the RLS algorithm, we conducted a parameter search over 1000 independent random trials. Each trial consisted of $T=25$ blocks of $S=2000$ samples for each parameter value (or parameter value combination). During the $j$ th block, only the $j$ th transmitter is active and only the channels in $\widehat{\mathbf{M}}_{r}^{(j)}$ are being updated, as shown in Fig. 4.3 .

For all of the parameter studies in Section 4.3 , the results are shown for a $5 \times 10$ element uniform rectangular array, operating at a center frequency $f_{c}=2.42 \mathrm{GHz}$ with a $100 \mathrm{MHz}$ bandwidth. The elements were spaced with half-wavelength spacing at the center frequency, and the array geometry is shown in Fig. 4.4. Each transmit 


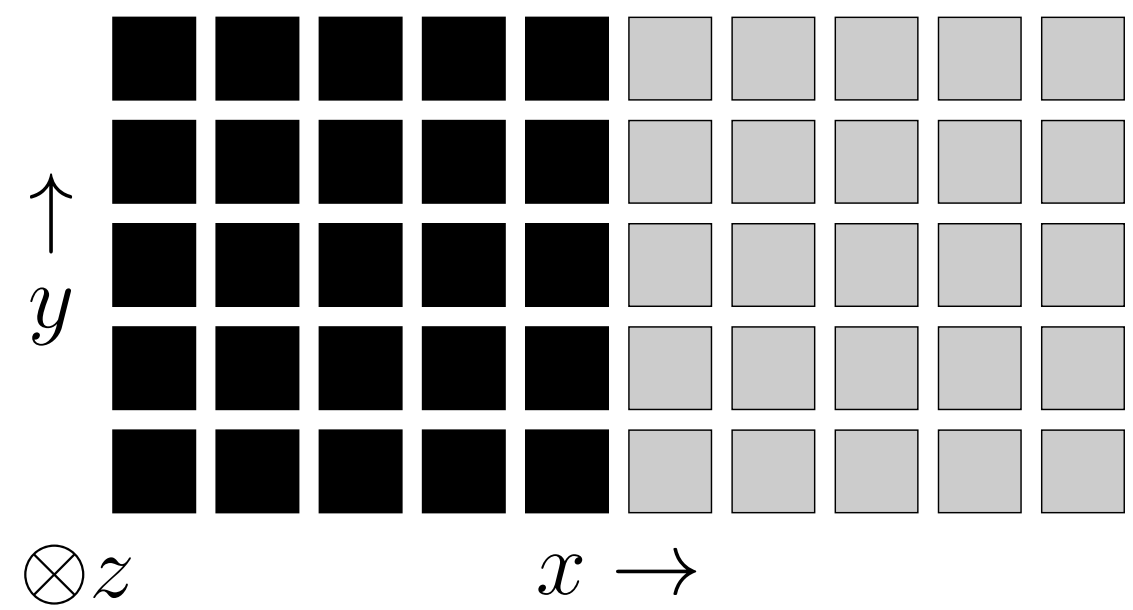

Figure 4.4: Layout of the ALSTAR array simulated in this work. Black and grey elements represent transmitting and receiving antennas, respectively. Taken from Chapter 2 .

channel had a dynamic range of $\eta_{t}=45 \mathrm{~dB}$ and each receive or reference channel had a dynamic range of $\eta_{r}=70 \mathrm{~dB}$. The fixed thermal noise power of the receive channels was $\sigma_{r}^{2}=-91 \mathrm{dBm}$, which corresponds to the Johnson-Nyquist noise plus a $3 \mathrm{~dB}$ noise figure. Each transmit and receive beamformer channel had $N_{t}=32$ taps and $N_{r}=32$ taps, respectively. The ideal mutual coupling channel was modeled using MATLAB's Antenna Toolbox at $N_{m}=32$ frequencies spanning the frequency range of interest. For each trial, the ideal mutual coupling model was perturbed by simulating the relative displacement of the antennas as might be caused by manufacturing tolerances or thermal deformations in the array, and applying the corresponding wideband fractional delay filter to each of the individual channels. The displacement of each antenna was modeled as a zero-mean Gaussian random variable with a standard deviation of $0.1 \mathrm{~mm}$ in both the $x$ and $y$ directions. The transmitted signal used for probing was a complex Gaussian random waveform with $1 \mathrm{~W}$ of transmit 
power. It was assumed that no received SoI was present at the array during mutual coupling channel estimation. In half of the trials, an external interference signal was directed at the array. Its angle of arrival was drawn uniformly from $\phi \in[0,2 \pi]$ and $\theta \in[-\pi / 3, \pi / 3]$, and its received power had a log-uniform distribution over the interval $[-101,-61] \mathrm{dBm}$ per antenna.

The parameter search for the LMS channel estimation algorithm considered 34 unique values of the learning rate $\mu_{m}$ on the range $[-30,3] \mathrm{dB}$. The value of the parameter that produced the smallest MSE was $\mu_{m}=-7 \mathrm{~dB}$. The RLS channel estimation had slightly better performance than the LMS algorithm in terms of final channel estimate MSE. While the RLS algorithm proved relatively insensitive to changes in $\lambda_{m}$ and $\delta_{m}^{-1}$ because the probe input signals for channel sounding were truly white and independent, the parameter search indicated that the optimal parameter values for the range of cases presented were $\lambda_{m}=1$ and $\delta_{m}^{-1}=10^{10}$.

Fig. 4.5 plots the convergence behavior of the SMI, LMS, and RLS channel estimation algorithms over 1000 trials as described above with the optimal parameter values for the LMS and RLS algorithms. Each line shows the total squared error of the estimated mutual coupling channel, having aggregated the channel errors at each sample index $n$ across all the separate channel estimation blocks. The error bars indicate the minimum and maximum total squared error across the trials. Although the SMI calculation is performed once in each block and not at each sample, we have plotted 


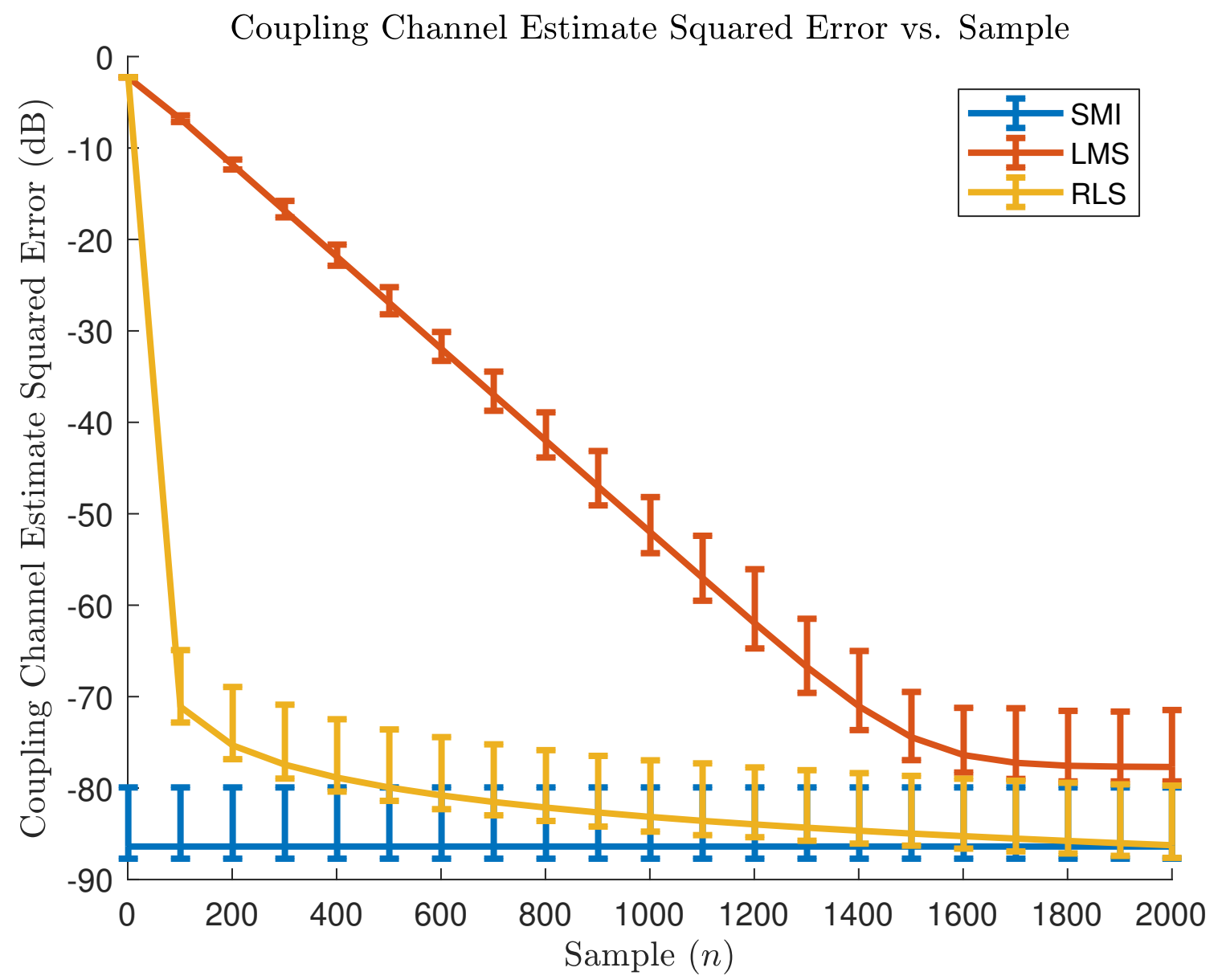

Figure 4.5: Results of the convergence performance experiment for the LMS and RLS algorithms using the optimal parameters identified in the parameter searches. The final squared error of the SMI method is included for comparison. The minimum, mean, and maximum squared error of the channel estimate over the 1000 trials is plotted.

the total aggregated estimation error for the SMI method across time for comparison with the other methods. As expected, the SMI method sets the performance limit for the other methods, which are stochastic approximations of the SMI method. The reduction in error provided by the RLS and SMI algorithms over the LMS algorithm is worthwhile. The LMS algorithm might find use in cases where the channel estimation performance requirements might be less stringent. 


\section{Table 4.1}

Channel Estimation Algorithm Computational Complexity

\begin{tabular}{c|l} 
Algorithm & Computational Complexity (FLOPs per Block) \\
\hline \hline SMI & $4 N_{m}^{2} S+8 R N_{m} S+4 N_{m} S+4 / 3 N_{m}^{3}+8 R N_{m}^{2}+10 N_{m}^{2}+12 R N_{m}+$ \\
& $38 / 3 N_{m}+18$ \\
\hline LMS & $16 R N_{m} S+8 N_{m} S+6 R S+15 S+11$ \\
\hline RLS & $35 N_{m}^{2} S+16 R N_{m} S+26 N_{m} S+6 S+11$
\end{tabular}

Table 4.1 compares the computational complexity of the SMI, LMS, and RLS channel estimation techniques in real floating point operations (FLOPs) over a single channel estimation block, because channel estimation is repeated sequentially for all $T$ transmitters as shown in Fig. 4.3. This analysis assumes that each complex addition costs 2 real FLOPs, each complex multiplication costs 6 real FLOPs [191], each complex division costs 11 real FLOPs [192], and each complex square root costs 15 real FLOPs [193]. The complexity analysis of the SMI method referenced [194, 195, 196, 197]. This work does not account for the cost of data networking and management between the transmitters and receivers when comparing algorithms. While the LMS algorithm has $\mathcal{O}\left(R N_{m} S\right)$ complexity, the performance advantage of the SMI algorithm is critical for overall system performance. Therefore, we accept the $\mathcal{O}\left(\max \left\{N_{m}^{2} S, R N_{m} S\right\}\right)$ complexity of the SMI channel estimation algorithm, which we use in the remainder of the simulations in this work. 


\subsection{Adaptive Beamforming for ALSTAR}

With a practical method of accurately estimating the coupling channel in hand, the task that remains is to further isolate the transmit and receive channels via adaptive transmit and receive beamforming. In Chapter 2, we demonstrated how transmit and receive adaptive beamforming are coupled problems and both contribute to EII for narrowband ALSTAR arrays. Here, we must formulate the adaptive beamforming problems to account for imperfect mutual coupling channel knowledge and potential

external interference. Considering (4.12) and 4.13), the adaptive receive beamformer must maintain high gain on the SoI while suppressing the remaining interference and noise terms. The transmit beamformer can similarly contribute to the isolation by reducing the incident power on the receive antennas in order to improve their sensitivity, while maintaining high gain in the direction of interest.

\subsubsection{SMI Receive Beamformer \& Wiener Optimal Transmit Beamformer}

Extending the adaptive transmit and receive beamforming concepts from Chapter 2 is not mathematically trivial, but it is conceptually straightforward. The work in Chapter 2 showed that alternately updating the transmit and receive beamformer 
using the Wiener optimal filter equations provided a means of maximizing EII. First let us consider the receive beamformer. The covariance matrix in the formulation of the Wiener optimal filter for the receive beamformer was the covariance matrix of the residual interference and noise after SIC. In that work, the receive covariance matrix was calculated using knowledge of the coupling matrix and system parameters, which made it impossible to account for the presence of external interference. However, because we have access to the received signals after SIC, it is simple to estimate the residual interference and noise covariance matrix as

$$
\mathbf{R}_{r r}=\frac{1}{S} \mathrm{C}^{N_{r}}\left(\mathbf{r}_{c}\right) \mathrm{C}^{N_{r}}\left(\mathbf{r}_{c}\right)^{\mathrm{H}}
$$

Using our wideband definition of the receive steering vector, we can formulate our SMI receive beamformer,

$$
\mathbf{b}_{r}=\alpha \mathbf{R}_{r r}^{-1} \mathbf{q}_{r}, \text { s.t. }\left\|\mathbf{b}_{r}\right\|^{2}=1
$$

which is the best approximation of the Wiener optimal receive beamformer from Chapter 2. Note that $\alpha$ is an arbitrary constant that allows us to meet the magnitude constraint. As any potential external interference is present in $\mathbf{r}_{c}$ and these signals only depend on an estimate of the mutual coupling matrix, 4.38 addresses all of the weaknesses of the adaptive beamforming formulation in the previous work. 
Next, we consider how we might approach the transmit beamforming problem. One of the key insights presented in Chapter 2 was that the residual noise and interference power could be expressed as a quadratic form with respect to the receive beamformer or the transmit beamformer. When factored as a quadratic form with respect to the transmit beamformer, the covariance matrix quantifies the contribution of each transmit channel to the received noise and residual SIC noise due to the observation channel noise. By updating based on this calculated covariance matrix, the adaptive transmit beamformer can reduce the components of the noise in the isolated received signal $y[n]$ that are effected by the transmit beamformer. Extending the definition of 
the transmit covariance matrix from narrowband to wideband, we obtain

$$
\begin{aligned}
& \underbrace{-1}_{t t} \widetilde{\mathbf{M}}_{t}^{*}\left[\begin{array}{ccc}
\left\|\mathbf{h}_{r}^{(1)}\right\|^{2} \mathbf{I}_{N_{m}} & & 0 \\
& \ddots & \\
0 & & \left\|\mathbf{h}_{r}^{(R)}\right\|^{2} \mathbf{I}_{N_{m}}
\end{array}\right] \widetilde{\mathbf{M}_{t}^{T}} \\
& \text { Rx Noise due to Tx Signal Self-Interference } \\
& +\underbrace{\eta_{r}^{-1} \operatorname{Diag}\left(\left[\begin{array}{c}
\left.\sum_{k=1}^{R}\left\|\mathbf{h}_{r}^{(k)} \circledast \mathbf{h}_{m}^{(k, 1)}\right\|^{2} \mathbf{1}_{N_{t}}\right] \\
\vdots \\
\left.\sum_{k=1}^{R}\left\|\mathbf{h}_{r}^{(k)} \circledast \mathbf{h}_{m}^{(k, T)}\right\|^{2} \mathbf{1}_{N_{t}}\right]
\end{array}\right)\right.}_{\text {SIC Observer Noise }} \\
& +\underbrace{\eta_{r}^{-1} \eta_{t}^{-1} \operatorname{Diag}\left(\left[\begin{array}{c}
\sum_{k=1}^{R}\left\|\mathbf{h}_{r}^{(k)}\right\|^{2}\left\|\mathbf{h}_{m}^{(k, 1)}\right\|^{2} \mathbf{1}_{N_{t}} \\
\vdots \\
\sum_{k=1}^{R}\left\|\mathbf{h}_{r}^{(k)}\right\|^{2}\left\|\mathbf{h}_{m}^{(k, T)}\right\|^{2} \mathbf{1}_{N_{t}}
\end{array}\right]\right.} \\
& \text { Rx Noise due to Tx Noise Self-Interference } \\
& +\underbrace{\frac{\sigma_{r}^{2}}{P_{t}} \mathbf{I}_{T \cdot N_{t}}}_{\text {Rx Thermal Noise }},
\end{aligned}
$$

where

$$
\widetilde{\mathbf{M}}_{t}=\left[\begin{array}{ccc}
\tilde{\mathrm{C}}^{N_{t}}\left(\left(\hat{\mathbf{h}}_{m}^{(1,1)}\right)^{T}\right) & \ldots & \tilde{\mathrm{C}}^{N_{t}}\left(\left(\hat{\mathbf{h}}_{m}^{(1, R)}\right)^{T}\right) \\
\vdots & \ddots & \vdots \\
\tilde{\mathrm{C}}^{N_{t}}\left(\left(\hat{\mathbf{h}}_{m}^{(T, 1)}\right)^{T}\right) & \cdots & \tilde{\mathrm{C}}^{N_{t}}\left(\left(\hat{\mathbf{h}}_{m}^{(T, R)}\right)^{T}\right)
\end{array}\right]
$$

is the estimated mutual coupling matrix that has been expanded to account for the $N_{t}$ taps per transmit channel. Note that $P_{t}$ is the total transmitted signal power from 
the array. With this definition for the transmit covariance matrix, we can calculate the Wiener optimal transmit beamformer,

$$
\mathbf{b}_{t}=\beta \mathbf{R}_{t t}^{-1} \mathbf{q}_{t}, \text { s.t. }\left\|\mathbf{b}_{t}\right\|^{2}=P_{t}
$$

where $\beta$ is an arbitrary constant that allows us to meet the total transmitted signal power constraint.

As shown in Fig. 4.3 , by repeatedly calculating the SMI adaptive receive beamformer over a block of samples via 4.37) and (4.38) and then using the resulting receive beamformer in updating the transmit beamformer via 4.39) and 4.41, we successfully mimic the alternating optimization procedure described in Chapter 2, while accounting for the possibility of external interference. The matrix inversions required for these adaptive beamforming schemes present potential implementation difficulties as they are computationally expensive. In the search for more computationally efficient adaptive beamforming solutions, we propose LMS and RLS implementations of the linearly-constrained minimum variance (LCMV) beamformer for adaptive receive beamforming. We use the Wiener optimal adaptive transmit beamformer throughout the rest of the work because generating an artificial signal with the correct statistics for an LMS or RLS adaptive transmit beamformer would be prohibitively expensive. At the end of this section we will compare the computational complexity and convergence performance of all of these adaptive techniques. 


\subsubsection{LMS LCMV Receive Beamformer}

The LMS LCMV beamformer implemented in this work was extended from the presentations in Chapters 2 and 6 of [167]. The challenge in adaptive beamforming within the LMS paradigm is to constrain the algorithm to avoid nulling out the SoI along with the noise and interference. To accomplish this, the LCMV beamformer consists of a default beamformer component that has maximum gain on the SoI and an adaptive beamformer component that operates on the received signals after they have been projected into the null-space of the default beamformer. For the receive beamformer, the null-space signal projection matrix $\mathbf{K}_{r} \in \mathbb{C}^{R \cdot N_{r} \times R \cdot N_{r}-1}$ is the last $R \cdot N_{r}-1$ columns of a Householder reflection matrix [198, 199, 200], such that

$$
\mathbf{K}_{r}=-\frac{q_{r}^{*}(1)}{\left|q_{r}(1)\right|}\left(\left[\begin{array}{l}
\mathbf{0}_{R \cdot N_{r}-1}^{T} \\
\mathbf{I}_{R \cdot N_{r}-1}
\end{array}\right]-2 \mathbf{v}_{r} \cdot \mathbf{v}_{r}^{\mathbf{H}}\left(2: R \cdot N_{r}\right)\right),
$$

where

$$
\mathbf{v}_{r}=\frac{\mathbf{q}_{r}+\left\|\mathbf{q}_{r}\right\| e^{j \angle q_{r}(1)}[1,0, \cdots, 0]^{T}}{\left\|\mathbf{q}_{r}+\right\| \mathbf{q}_{r}\left\|e^{j\left\llcorner q_{r}(1)\right.}[1,0, \cdots, 0]^{T}\right\|} .
$$

Given this projection matrix, the adaptive beamformer becomes

$$
\mathbf{b}_{r}[n]=\gamma\left(\frac{1}{\left\|\mathbf{q}_{r}\right\|} \mathbf{q}_{r}-\mathbf{K}_{r} \mathbf{b}_{r, a}[n]\right), \text { s.t. }\left\|\mathbf{b}_{r}\right\|^{2}=1,
$$


where $\mathbf{b}_{r, a}[n] \in \mathbb{C}^{R \cdot N_{r}-1}$ is the portion of the beamformer that is updated by the adaptive algorithm and $\gamma$ is an arbitrary constant that allows us to enforce the magnitude constraint. The error signal is simply the output of the receive beamformer,

$$
e_{r}[n]=y[n]
$$

The receive beamformer operates on the signals after SIC, $r_{c}^{(k)}$, and so the update equation for the adaptive portion of the receive beamformer is

$$
\mathbf{b}_{r, a}[n+1]=\mathbf{b}_{r, a}[n]+\frac{\mu_{r}}{\left\|\mathbf{u}_{r}[n]\right\|^{2}} \mathbf{u}_{r}[n] e_{r}^{*}[n]
$$

where

$$
\mathbf{u}_{r}[n]=\mathbf{K}_{r}^{\mathrm{H}} \mathrm{C}^{N_{r}}\left(\mathbf{r}_{c}\right)[n] .
$$

\subsubsection{RLS LCMV Receive Beamformer}

The adaptive LMS LCMV receive beamforming scheme described above can be extended to the RLS paradigm as well. The whitened input vector, after the received signals have been projected into the null space of the SoI steering vector, is

$$
\mathbf{c}_{r}[n]=\lambda_{r}^{-1} \mathbf{P}_{r}[n] \mathbf{u}_{r}[n]
$$


The initial input inverse correlation matrix $\mathbf{P}_{r}[0]=\delta_{r}^{-1} \mathbf{I}_{R \cdot N_{r}-1}$, and the tuning parameter $\lambda_{r} \in(0,1]$. We define a scaled version of the whitened input vector for convenience,

$$
\mathbf{k}_{r}[n]=\left(1+\mathbf{u}_{r}^{\mathrm{H}}[n] \mathbf{c}_{r}[n]\right)^{-1} \mathbf{c}_{r}[n]
$$

Thus, the update equation for the adaptive portion of the receive beamformer is

$$
\mathbf{b}_{r, a}[n+1]=\mathbf{b}_{r, a}[n]+\mathbf{k}_{r}[n] e_{r}^{*}[n]
$$

where $e_{r}[n]$ is defined in 4.45 and the full receive beamformer is calculated as in (4.44). Finally, the inverse correlation matrix update equation becomes

$$
\mathbf{P}_{r}[n+1]=\lambda_{r}^{-1}\left(\mathbf{P}_{r}[n]-\mathbf{k}_{r}[n] \mathbf{u}_{r}^{\mathbf{H}}[n] \mathbf{P}_{r}[n]\right)
$$

\subsubsection{Performance Comparison Results}

All of the adaptive beamforming techniques were evaluated under experimental conditions similar to those described in Section 4.3.4. In these simulations, both a transmitted and received SoI were present. The received SoI was an independent random QPSK waveform with a random received power that had a log-uniform distribution over the interval $[-101,-61] \mathrm{dBm}$ per antenna. The transmitted signal was also an independent random QPSK waveform with total transmitted power $P_{t}=25 \mathrm{~W}$. The 
transmitted signal and received SoI had the same angle of departure or angle of arrival, in order to simulate an FD communications link. Unless otherwise noted, the angles $\phi$ and $\theta$ were drawn uniformly from the ranges $[0,2 \pi]$ and $[-\pi / 3, \pi / 3]$, respectively. 100 trials of each parameter value or parameter value pair were run for the beamformer parameter tuning. The LMS receive beamformer was relatively insensitive to changes in the convergence parameter. The optimal convergence parameter value was found to be $\mu_{r}=2.5 \mathrm{~dB}$. The RLS beamformer was relatively insensitive to the memory parameter $\lambda_{r}$, but it was sensitive to the regularization parameter $\delta_{r}^{-1}$. The optimal parameter values were $\delta_{r}^{-1}=10^{13}$ and $\lambda_{r}=0.999$.

Fig. 4.6(a) plots the EII achieved using the SMI, LMS, and RLS adaptive receive beamforming algorithms in concert with the Wiener optimal transmit beamformer over 10 trials at broadside (i.e. $\phi=0$ and $\theta=0$ ) with the simulation parameters described above. The performance of the array is plotted over 5 blocks during the channel access and adaptive beamforming period shown in Fig. 4.3 in order to show how the transmit and receive beamformers work together to create isolation. On average in the final block, the SMI, LMS, and RLS beamforming methods were able to achieve an EII of $164.6 \mathrm{~dB}, 151.4 \mathrm{~dB}$, and $162.8 \mathrm{~dB}$, respectively. The EII performance of the RLS beamformer matched the SMI beamformer closely. While the LMS beamformer did not match the EII performance of the SMI and RLS receive beamformers, the LMS beamformer still performed well. For comparison, the ideal narrowband EII at broadside calculated according to the methods in Chapter 2 was 


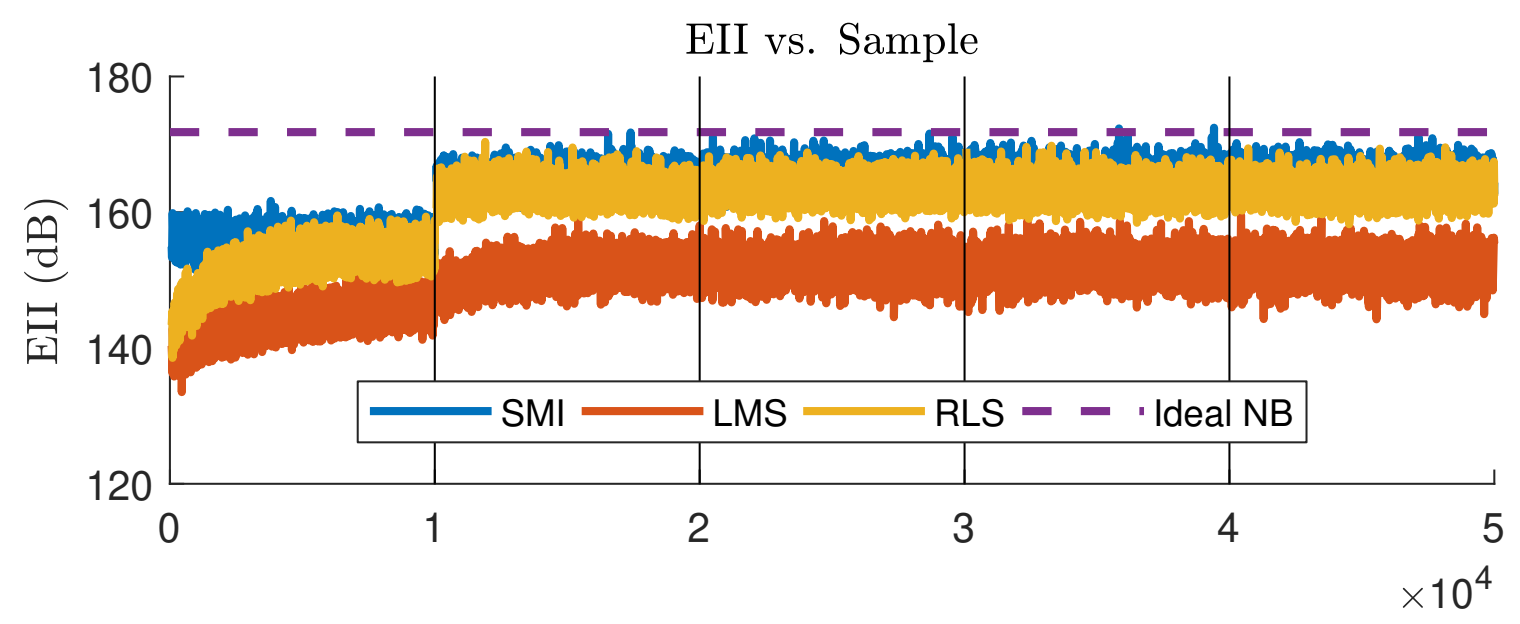

(a)

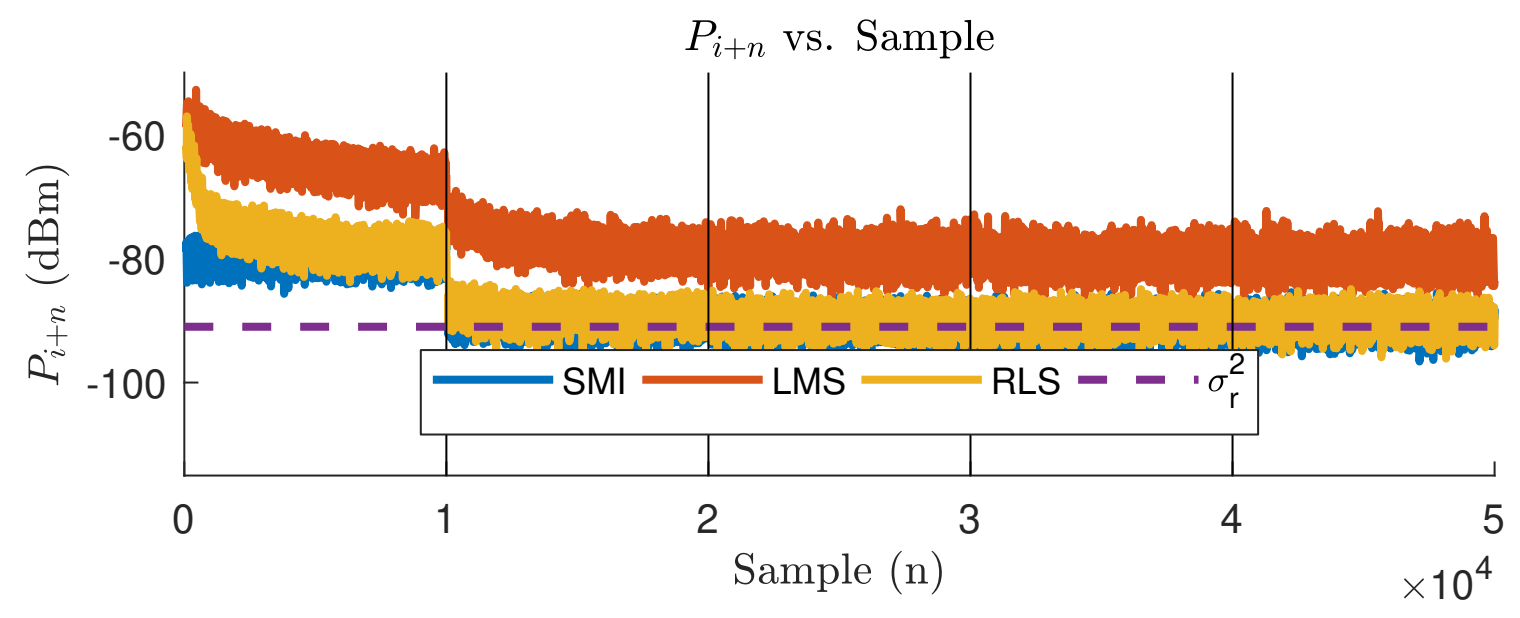

(b)

Figure 4.6: (a) Average EII and (b) $P_{i+n}$ at broadside plotted across 5 blocks for SMI, LMS, and RLS adaptive receive beamforming, respectively. The adaptive transmit beamformer in 4.41) was used with all the adaptive receive beamforming methods. The EII achieved at broadside by the ideal narrowband (NB) beamforming technique described in Chapter 2 and the thermal noise power $\sigma_{r}^{2}$ are plotted for comparison.

$171.8 \mathrm{~dB}$ with an ideal mutual coupling channel.

Fig. 4.6(b) plots the residual interference and noise power $P_{i+n}$ at broadside achieved using the SMI, LMS, and RLS adaptive receive beamforming algorithms with the 
Wiener optimal transmit beamformer. In the last block, the SMI, LMS, and RLS receive beamforming algorithms were able to reduce the average residual interference and noise power $P_{i+n}$ to $-90.0 \mathrm{dBm},-79.5 \mathrm{dBm}$, and $-89.4 \mathrm{dBm}$, respectively. As the average minimum noise power was $\sigma_{r}^{2}=-91 \mathrm{dBm}$ and the ideal narrowband noise power at broadside achieved in Chapter 2 was $-90.8 \mathrm{dBm}$, it appears that the SMI adaptive receive beamformer most closely achieved the ideal performance as expected.

Fig. 4.7(a) plots the average EII achieved by the SMI, LMS, and RLS beamformers in the last block over 101 evenly spaced look angles that span the azimuth scan space (i.e. $\phi=0$ and $\theta \in[-\pi / 3, \pi / 3]$ ). The ideal narrowband EII achieved by the method described in Chapter 2 is also plotted for comparison. Like at broadside, the SMI and RLS methods match closely in terms of EII, while the LMS beamforming method achieved approximately $10.1 \mathrm{~dB}$ less EII than the SMI method across the azimuth scan space. Fig. 4.7(b) plots the average $P_{i+n}$ at the same azimuth scan angles as shown for EII. The SMI beamformer reduced the residual interference and noise power essentially to the thermal noise power of the receive channels. The LMS and RLS receive beamformers achieved approximately $9.1 \mathrm{~dB}$ and $0.7 \mathrm{~dB}$ higher residual interference and noise powers than the SMI receive beamformer when averaged across the azimuth scan space.

Fig. 4.8(a) and Fig. 4.8(b) plots the EII and $P_{i+n}$ performance of the ALSTAR array when an external interference QPSK signal was directed at the array. In each trial, 


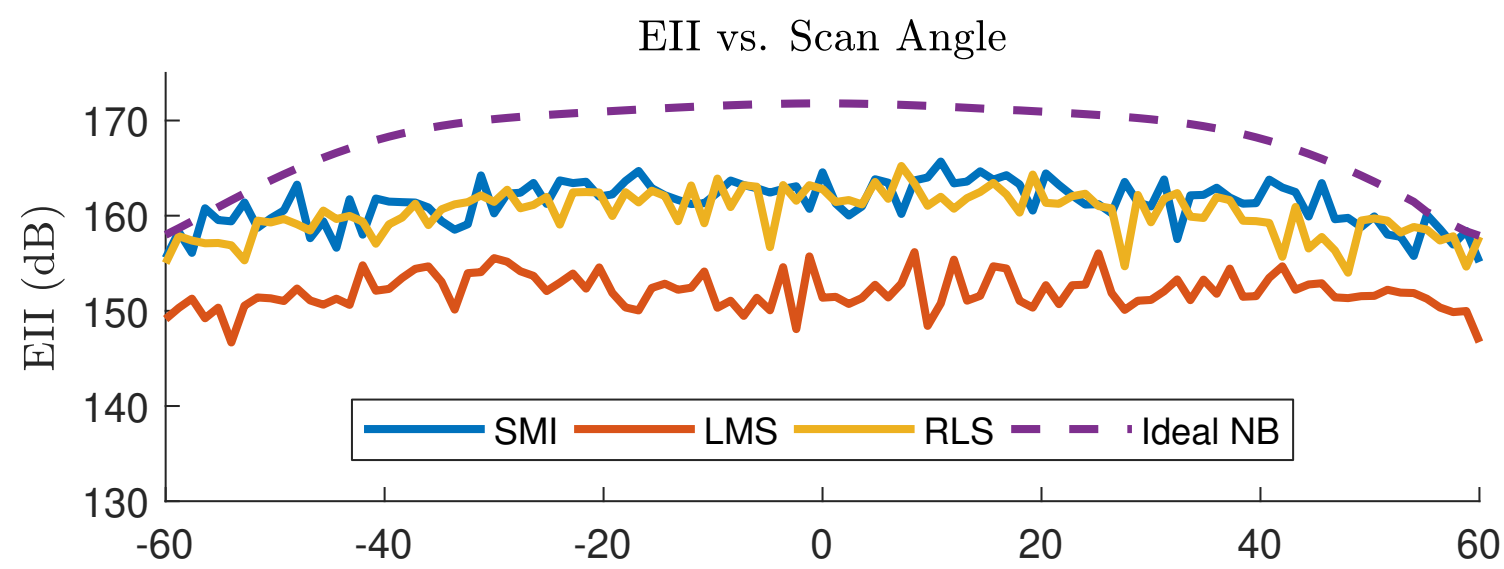

(a)

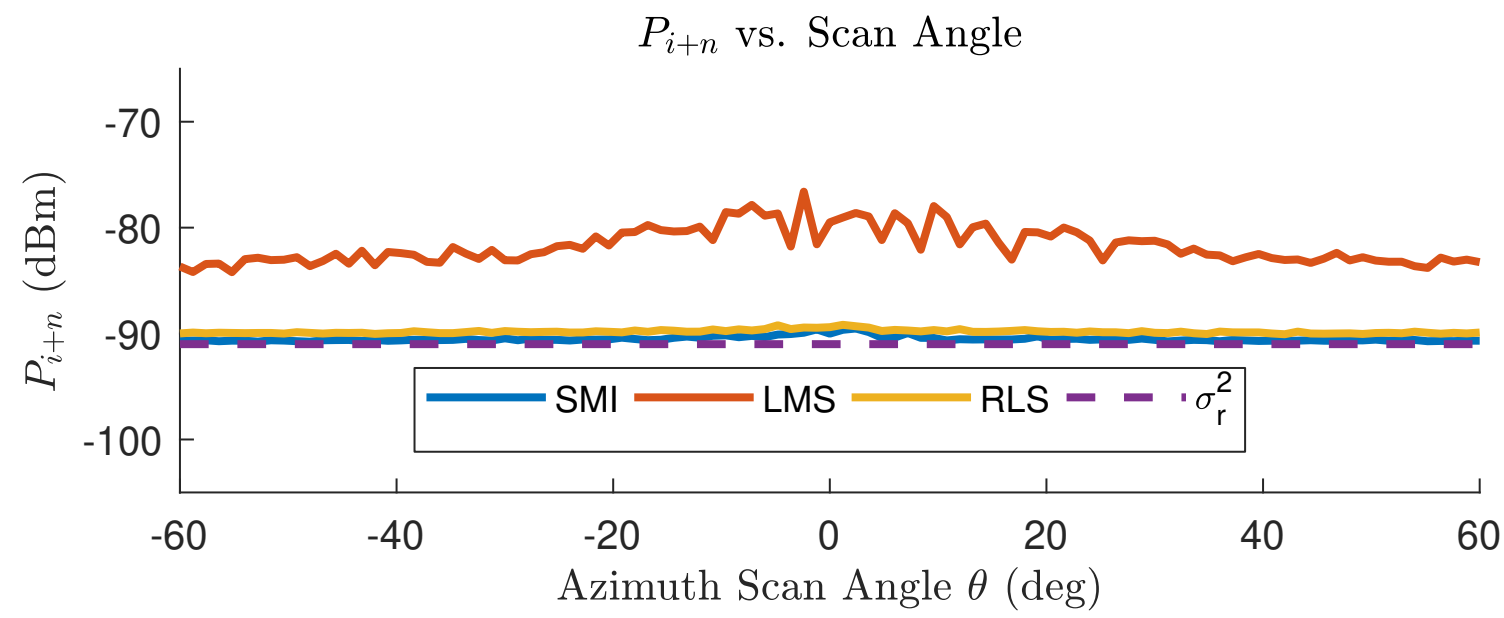

(b)

Figure 4.7: (a) Average EII and (b) $P_{i+n}$ plotted across 101 evenly spaced azimuth scan angles $(\phi=0$ and $\theta \in[-\pi / 3, \pi / 3])$ for the SMI, LMS, and RLS receive beamformers in concert with the Wiener optimal transmit beamformer. External interference was absent from these trials. 10 trials were conducted at each scan angle.

its angle of arrival was drawn uniformly from $\phi \in[0,2 \pi]$ and $\theta \in[-\pi / 3, \pi / 3]$, and its received power had a log-uniform distribution over the interval $[-101,-61] \mathrm{dBm}$ per antenna. While both the EII and $P_{i+n}$ performance does suffer under the presence of external interference, the adaptive beamforming algorithms still perform admirably in isolating the transmit and receive channels. 


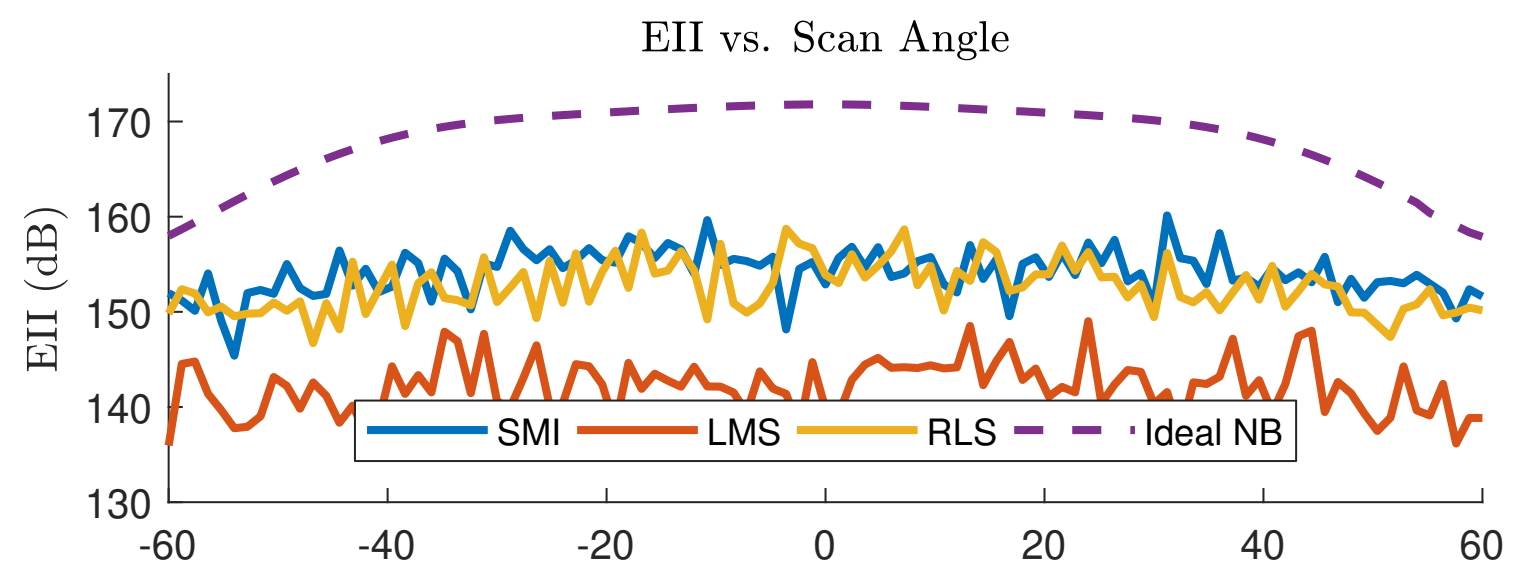

(a)

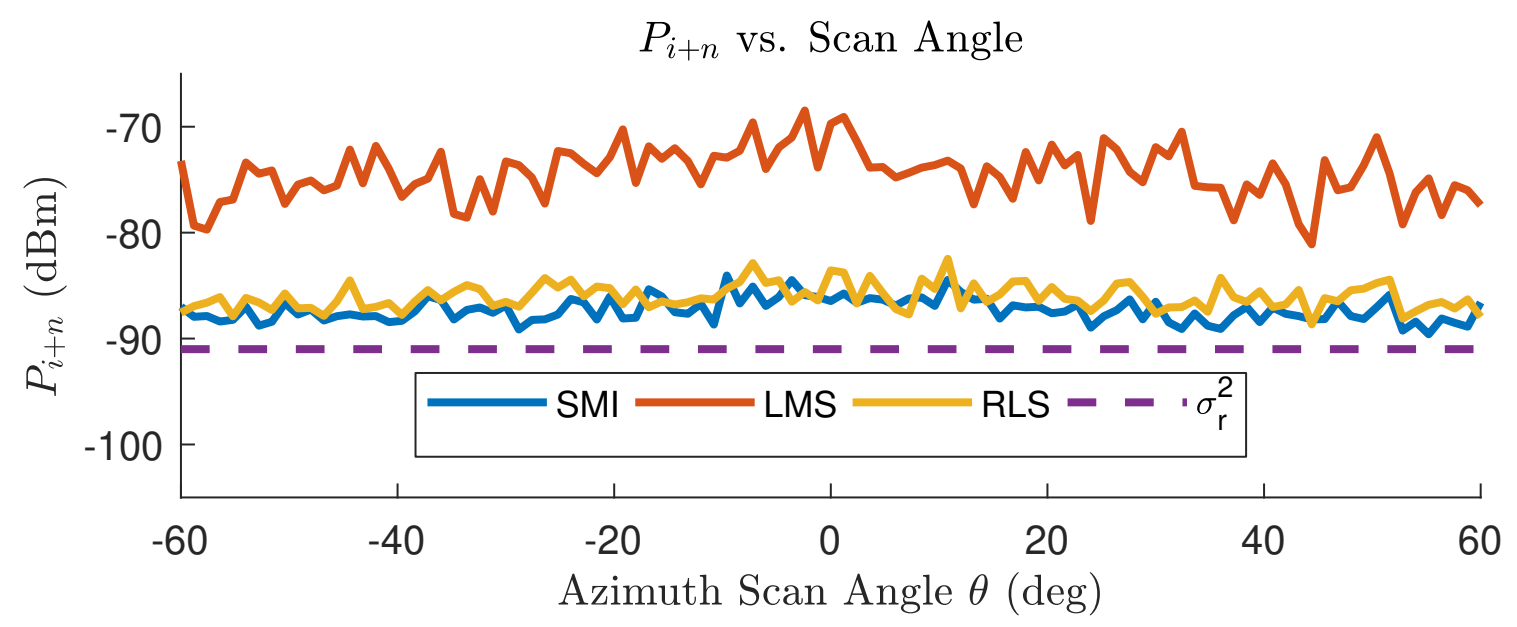

(b)

Figure 4.8: (a) Average EII and (b) $P_{i+n}$ plotted across 101 evenly spaced azimuth scan angles $(\phi=0$ and $\theta \in[-\pi / 3, \pi / 3])$ for the SMI, LMS, and RLS receive beamformers in concert with the Wiener optimal transmit beamformer. External interference was present in these trials. 10 trials were conducted at each scan angle.

Fig. 4.9 compares the computational complexity of the ALSTAR system during the channel access and adaptive beamforming period shown in Fig. 4.3 when implemented with the SMI, LMS, and RLS adaptive receive beamforming algorithms. In this analysis, we have amortized the cost of the transmit beamforming operations and other operations not repeated with each received sample across the period of a single 

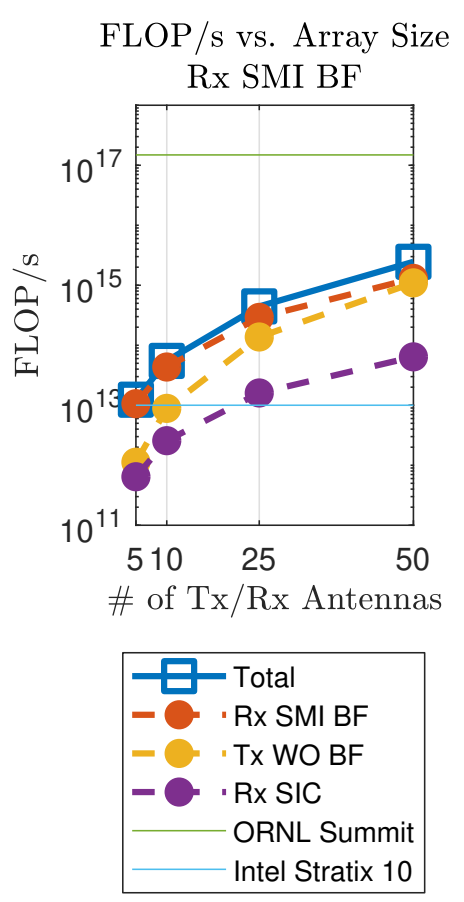

(a)
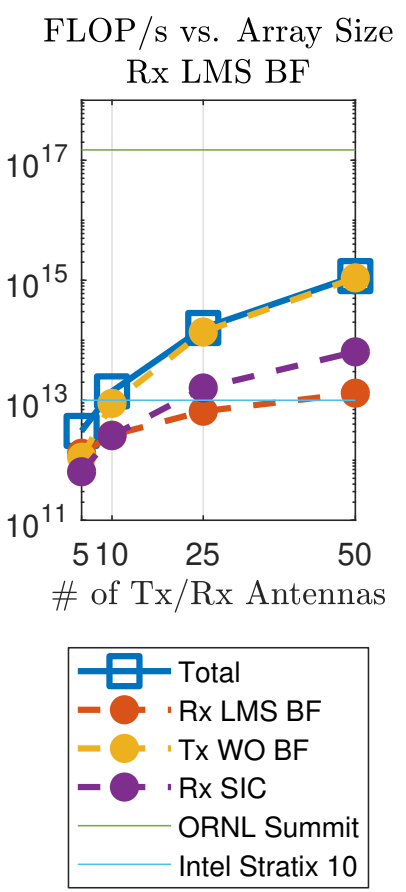

(b)
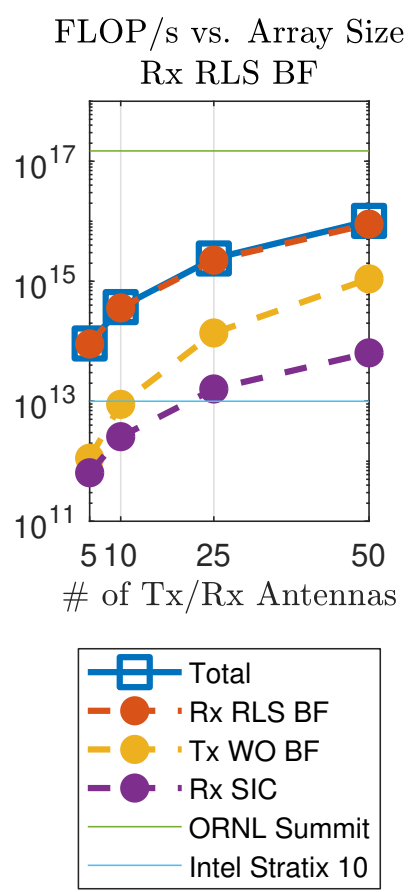

(c)

Figure 4.9: Computational complexity of the solutions based on the (a) SMI, (b) LMS and (c) RLS adaptive receive beamforming algorithms. The total computational complexity is given and it is also broken down into receive beamforming, transmit beamforming, and SIC complexities. For reference, the maximum computational output of a single Intel Stratix 10 FPGA and the Summit supercomputer at Oak Ridge National Laboratory are also plotted.

receive beamformer update block. Like in Section 4.3.4, the complexity analysis of the SMI and Wiener optimal methods referenced [194, 195, 196, 197]. In order to place the complexity of the proposed algorithms in context, we have also plotted the maximum computational capacity of the Intel Stratix 10 FPGA (one of the highest performance FPGAs currently available) [201] and Oak Ridge National Laboratory's Summit supercomputer which was the most powerful supercomputer in the world as of November 2019 according to Top500.org [202]. The first thing to note from these plots is the substantial computational complexity of the proposed algorithms. 
Fig. 4.9 (a) plots the computational requirements of the ALSTAR architecture when the SMI adaptive receive beamformer is used, and shows that the receive beamformer dominates the cost. As shown in Fig. 4.9(b), when the LMS beamforming algorithm is used, transmit beamforming and SIC dominate the complexity. In Fig. 4.9(c), the cost of the RLS beamforming algorithm dominates. Although LMS adaptive beamforming does not perform as well as the SMI or RLS methods, the reduced computational complexity it offers may make implementing ALSTAR feasible with the available computational hardware. For example, according to Fig. 4.9(b) an ALSTAR system with 10 transmit and receive antennas could theoretically be implemented using 2 Intel Stratix 10 FPGAs. Reducing the bandwidth of interest would also reduce the computational complexity and may make slightly larger arrays more feasible.

\subsection{Conclusion}

This work presented several mutual coupling channel estimation and adaptive beamforming techniques and analyzed their ability to work in concert to create isolation in an ALSTAR array. This work represents the most realistic signal model for the ALSTAR array to date, in that it includes the effects of the presence of the SoI and external interference in the received signals. We considered methods that could potentially enable an ALSTAR array to operate in real-time, achieving STAR on a fully digital and highly reconfigurable platform. 
There are a number of avenues for extending the work presented here. The largest drivers of computational complexity in the proposed architecture are adaptive transmit beamforming and SIC. Exploring methods for reducing the computational complexity of these parts of the architecture could be the key to reducing the computational load to more realistic levels. Additionally, subarray adaptive beamforming methods could offer a significant reduction in computational complexity without significant reduction in EII, enabling much larger ALSTAR arrays. Extending the ALSTAR architecture to MIMO operation would make the ALSTAR technology relevant to an imporant and rapidly expanding portion of the wireless communications and sensing market. Finally, implementation and validation of the proposed techniques on hardware remains a critical step in the development of the ALSTAR architecture. 



\section{Chapter 5}

\section{Conclusion}

STAR and IBFD are widely studied problems in the fields of wireless communications and sensing. There have been many different systems proposed in order to transmit and receive meaningful signals in the same time slot and frequency band. This dissertation focused on the ALSTAR architecture, proposed by Doane et al. in [1].

Chapter 2 presented an idealized adaptive digital beamforming and self-interference cancellation technique for the ALSTAR array architecture that optimized the EII metric. The signal model and resulting beamformer optimization method accounted for the limited dynamic range of the transmit and receive channels of the array and arose naturally out of the structure of the expression for EII. The results strongly suggested 
that adaptive digital beamforming and self-interference cancellation could provide incredibly high transmit/receive isolation in the ALSTAR architecture by exploiting the coupled nature of the adaptive transmit and receive beamforming problems.

Chapter 3 considered the narrowband imaging performance of the ALSTAR architecture in terms of the CRLB and BCRB on the direction of arrival estimation error for a return from a point target. That scenario was then used to motivate an adaptive beamforming technique that maximized the CRLB at a sampled set of look angles within the scan space of the array. The BCRB was used to quantify the total performance of the array across the scan space so that an optimal transmit/receive aperture partition could be found using a GA.

Chapter 4 presented SMI, LMS, and RLS mutual coupling channel estimation and adaptive beamforming methods that are better suited to practical implementation than the results presented in Chapter 2. The proposed methods produced high EII in realistic simulations, bolstering the claim that the ALSTAR architecture is a strong approach to achieving STAR. Furthermore, the computational complexity of the various components of the proposed algorithms are highlighted in order to direct future efforts at increasing algorithmic efficiency.

Together, this dissertation represents a significant contribution to the understanding 
of the capabilities of the ALSTAR architecture in terms of EII and imaging performance. This work has paved the way forward for continued development by exploring the fundamental limitations of the ALSTAR architecture. This work also takes steps towards practical implementations of the ALSTAR architecture by making the transmit/receive aperture partition optimization feasible during array design and by leveraging practical adaptive beamforming and mutual coupling channel estimation methods for the ALSTAR problem.

\subsection{Future Work}

There is a great deal of work that remains in the development of the ALSTAR architecture. Continuing to improve the isolation performance and algorithmic efficiency of the adaptive beamforming and mutual coupling estimation techniques will be a critical part of practical ALSTAR implementations. Extending the DOA estimation results of Chapter 3 to wideband signal models would be important for many practical applications. As the ALSTAR technology continues to develop, it will be important to implement the architecture and verify its performance beyond the work done by Doane et al. in [6]. While this work will start in a laboratory setting, it will be important to integrate this technology into actual radar and communications systems for field testing in order to discover and overcome issues that arise with practical deploy-

ment. In order to facilitate ALSTAR development and testing across the community, 
it might be worthwhile to develop a well-documented, modular, extendable ALSTAR array using commonly available software-defined radios. This would significantly reduce the barrier to entry for those interested in the ALSTAR technology for their own applications. 


\section{References}

[1] J. P. Doane, K. E. Kolodziej, and B. T. Perry, "Simultaneous transmit and receive with digital phased arrays," in Proc. 2016 IEEE International Symposium on Phased Array Systems and Technology, Waltham, MA, USA, Oct 2016.

[2] S. Russell and P. Norvig, Artificial Intelligence: A Modern Approach, 3rd ed. Pearson, 2009.

[3] A. Sabharwal, P. Schniter, D. Guo, D. W. Bliss, S. Rangarajan, and R. Wichman, "In-band full-duplex wireless: Challenges and opportunities," IEEE Journal on Selected Areas in Communications, vol. 32, no. 9, pp. 1637-1652, Sept 2014 .

[4] K. E. Kolodziej, B. T. Perry, and J. S. Herd, "In-band full-duplex technology: Techniques and systems survey," IEEE Transactions on Microwave Theory and Techniques, pp. 1-17, 2019. 
[5] C. D. Nwankwo, L. Zhang, A. Quddus, M. A. Imran, and R. Tafazolli, "A survey of self-interference management techniques for single frequency full duplex systems," IEEE Access, vol. 6, pp. 30 242-30 268, 2018.

[6] J. P. Doane, K. E. Kolodziej, and B. T. Perry, "Simultaneous transmit and receive performance of an 8-channel digital phased array," in Proc. 2017 IEEE International Symposium on Antennas and Propagation 8 USNC/URSI National Radio Science Meeting, San Diego, CA, USA, July 2017.

[7] J. G. Proakis and M. Salehi, Digital Communications, 5th ed. McGraw-Hill, 2008.

[8] T. Snow, C. Fulton, and W. J. Chappell, "Multi-antenna near field cancellation duplexing for concurrent transmit and receive," in 2011 IEEE MTT-S International Microwave Symposium, June 2011, pp. 1-4.

[9] —_, "Transmit-receive duplexing using digital beamforming system to cancel self-interference," IEEE Transactions on Microwave Theory and Techniques, vol. 59, no. 12, pp. 3494-3503, Dec 2011.

[10] E. Aryafar, M. A. Khojastepour, K. Sundaresan, S. Rangarajan, and M. Chiang, "MIDU: enabling MIMO full duplex," in Proceedings of the 18th Annual International Conference on Mobile Computing and Networking. ACM, 2012, pp. 257-268. 
[11] K. E. Kolodziej, P. T. Hurst, A. J. Fenn, and L. I. Parad, "Ring array antenna with optimized beamformer for simultaneous transmit and receive," in Proceedings of the 2012 IEEE International Symposium on Antennas and Propagation, July 2012, pp. 1-2.

[12] A. T. Wegener and W. J. Chappell, "Coupled antenna scheme using filter design techniques and tunable resonators to show simultaneous transmit and receive," in 2013 IEEE MTT-S International Microwave Symposium Digest (MTT), June 2013, pp. 1-4.

[13] W. F. Moulder, B. T. Perry, and J. S. Herd, "Wideband antenna array for simultaneous transmit and receive (STAR) applications," in 2014 IEEE Antennas and Propagation Society International Symposium (APSURSI), July 2014, pp. $243-244$.

[14] A. T. Wegener, "Broadband near-field filters for simultaneous transmit and receive in a small two-dimensional array," in 2014 IEEE MTT-S International Microwave Symposium (IMS2014), June 2014, pp. 1-3.

[15] T. Dinc and H. Krishnaswamy, "A T/R antenna pair with polarization-based reconfigurable wideband self-interference cancellation for simultaneous transmit and receive," in 2015 IEEE MTT-S International Microwave Symposium, May 2015, pp. 1-4. 
[16] M. A. Elmansouri, A. J. Kee, and D. S. Filipovic, "Wideband antenna array for simultaneous transmit and receive (STAR) applications," IEEE Antennas and Wireless Propagation Letters, vol. 16, pp. 1277-1280, 2017.

[17] E. A. Etellisi, M. A. Elmansouri, and D. S. Filipovic, "Wideband monostatic co-polarized co-channel simultaneous transmit and receive (C-STAR) broadside circular array antenna," IEEE Transactions on Antennas and Propagation, pp. $1-1,2018$.

[18] G. Kwon, J. Park, D. Kim, and K. C. Hwang, "Optimization of a sharedaperture dual-band transmitting/receiving array antenna for radar applications," IEEE Transactions on Antennas and Propagation, vol. 65, no. 12, pp. 7038-7051, Dec 2017.

[19] R. Lian, T. Shih, Y. Yin, and N. Behdad, "A high-isolation, ultra-wideband simultaneous transmit and receive antenna with monopole-like radiation characteristics," IEEE Transactions on Antennas and Propagation, vol. 66, no. 2, pp. 1002-1007, Feb 2018.

[20] E. A. Alwan, A. Hovsepian, and J. L. Volakis, "Ultra-wideband dual polarization arrays with collocated elements for high isolation simultaneous transmit and receive systems," in 2016 IEEE International Symposium on Phased Array Systems and Technology (PAST), Oct 2016, pp. 1-3. 
[21] E. A. Etellisi, M. A. Elmansouri, and D. S. Filipovic, "Wideband simultaneous transmit and receive (STAR) circular array system," in 2016 IEEE International Symposium on Phased Array Systems and Technology (PAST), Oct 2016, pp. $1-5$.

[22] E. Yetisir, C. Chen, and J. L. Volakis, "Wideband low profile multiport antenna with omnidirectional pattern and high isolation," IEEE Transactions on Antennas and Propagation, vol. 64, no. 9, pp. 3777-3786, Sept 2016.

[23] J. Ha, M. A. Elmansouri, P. V. Prasannakumar, and D. S. Filipovic, "Monostatic co-polarized full-duplex antenna with left- or right-hand circular polarization," IEEE Transactions on Antennas and Propagation, vol. 65, no. 10, pp. 5103-5111, Oct 2017.

[24] K. Wei and B. Zhu, "The novel W parasitic strip for the circularly polarized microstrip antennas design and the mutual coupling reduction between them," IEEE Transactions on Antennas and Propagation, pp. 1-1, 2018.

[25] K. Nagai, Y. Mikuni, and H. Iwasaki, "A mobile radio antenna system having a self-diplexing function," IEEE Transactions on Vehicular Technology, vol. 28, no. 4, pp. 338-345, Nov 1979.

[26] H. Nawaz and I. Tekin, "Double-differential-fed, dual-polarized patch antenna with 90 dB interport RF isolation for a $2.4 \mathrm{GHz}$ in-band full-duplex transceiver," 
IEEE Antennas and Wireless Propagation Letters, vol. 17, no. 2, pp. 287-290, Feb 2018.

[27] G. Makar, N. Tran, and T. Karacolak, "A high-isolation monopole array with ring hybrid feeding structure for in-band full-duplex systems," IEEE Antennas and Wireless Propagation Letters, vol. 16, pp. 356-359, 2017.

[28] X. Wang, W. Che, W. Yang, W. Feng, and L. Gu, "Self-interference cancellation antenna using auxiliary port reflection for full-duplex application," IEEE Antennas and Wireless Propagation Letters, vol. 16, pp. 2873-2876, 2017.

[29] K. Iwamoto, M. Heino, K. Haneda, and H. Morikawa, "Design of an antenna decoupling structure for an inband full-duplex collinear dipole array," IEEE Transactions on Antennas and Propagation, vol. 66, no. 7, pp. 3763-3768, July 2018.

[30] M. Heino, S. N. Venkatasubramanian, C. Icheln, and K. Haneda, "Design of wavetraps for isolation improvement in compact in-band full-duplex relay antennas," IEEE Transactions on Antennas and Propagation, vol. 64, no. 3, pp. 1061-1070, March 2016.

[31] E. Everett, A. Sahai, and A. Sabharwal, "Passive self-interference suppression for full-duplex infrastructure nodes," IEEE Transactions on Wireless Communications, vol. 13, no. 2, pp. 680-694, February 2014. 
[32] E. Foroozanfard, E. D. Carvalho, and G. F. Pedersen, "Design and evaluation of full-duplex terminal antennas in realistic user scenarios," IEEE Antennas and Wireless Propagation Letters, vol. 16, pp. 1851-1854, 2017.

[33] E. Ahmed, A. M. Eltawil, Z. Li, and B. A. Cetiner, "Full-duplex systems using multireconfigurable antennas," IEEE Transactions on Wireless Communications, vol. 14, no. 11, pp. 5971-5983, Nov 2015.

[34] E. A. Etellisi, M. A. Elmansouri, and D. S. Filipovic, "Wideband multimode monostatic spiral antenna STAR subsystem," IEEE Transactions on Antennas and Propagation, vol. 65, no. 4, pp. 1845-1854, April 2017.

[35] P. V. Prasannakumar, M. A. Elmansouri, and D. S. Filipovic, "Broadband reflector antenna with high isolation feed for full-duplex applications," IEEE Transactions on Antennas and Propagation, vol. 66, no. 5, pp. 2281-2290, May 2018.

[36] A. Hovsepian, E. A. Alwan, and J. L. Volakis, "Wideband scanning array of spiral antennas for simultaneous transmit and receive (STAR)," in 2017 IEEE International Symposium on Antennas and Propagation USNC/URSI National Radio Science Meeting, July 2017, pp. 487-488.

[37] A. T. Wegener and W. J. Chappell, "High isolation in antenna arrays for simultaneous transmit and receive," in 2013 IEEE International Symposium on Phased Array Systems and Technology, Oct 2013, pp. 593-597. 
[38] A. Kord, D. L. Sounas, and A. Al, "Magnet-less circulators based on spatiotemporal modulation of bandstop filters in a delta topology," IEEE Transactions on Microwave Theory and Techniques, vol. 66, no. 2, pp. 911-926, Feb 2018.

[39] S. K. Cheung, T. P. Halloran, W. H. Weedon, and C. P. Caldwell, "MMICbased quadrature hybrid quasi-circulators for simultaneous transmit and receive," IEEE Transactions on Microwave Theory and Techniques, vol. 58, no. 3, pp. 489-497, March 2010.

[40] S. M. Duffy, G. A. Brigham, and J. S. Herd, "Integrated compensation network for low mutual coupling of planar microstrip antenna arrays," in 2007 IEEE Antennas and Propagation Society International Symposium, June 2007, pp. $1389-1392$.

[41] C. Cox and E. Ackerman, "Demonstration of a single-aperture, full-duplex communication system," in 2013 IEEE Radio and Wireless Symposium, Jan 2013, pp. $148-150$.

[42] J. Zhou, T. Chuang, T. Dinc, and H. Krishnaswamy, "Integrated wideband selfinterference cancellation in the RF domain for FDD and full-duplex wireless," IEEE Journal of Solid-State Circuits, vol. 50, no. 12, pp. 3015-3031, Dec 2015.

[43] K. E. Kolodziej, J. G. McMichael, and B. T. Perry, "Multitap RF canceller for in-band full-duplex wireless communications," IEEE Transactions on Wireless Communications, vol. 15, no. 6, pp. 4321-4334, June 2016. 
[44] S. J. Watt, E. A. Alwan, and J. L. Volakis, "Cascaded network analysis of a wideband RF self-interference cancellation (RF-SIC) filter for STAR systems," in 2016 IEEE International Symposium on Antennas and Propagation (APSURSI), June 2016, pp. 2117-2118.

[45] Y. Choi and H. Shirani-Mehr, "Simultaneous transmission and reception: Algorithm, design and system level performance," IEEE Transactions on Wireless Communications, vol. 12, no. 12, pp. 5992-6010, December 2013.

[46] Y. Liu, P. Roblin, X. Quan, W. Pan, S. Shao, and Y. Tang, "A full-duplex transceiver with two-stage analog cancellations for multipath self-interference," IEEE Transactions on Microwave Theory and Techniques, vol. 65, no. 12, pp. 5263-5273, Dec 2017.

[47] L. Laughlin, M. A. Beach, K. A. Morris, and J. L. Haine, "Electrical balance duplexing for small form factor realization of in-band full duplex," IEEE Communications Magazine, vol. 53, no. 5, pp. 102-110, May 2015.

[48] D. Liu, Y. Shen, S. Shao, Y. Tang, and Y. Gong, "On the analog self-interference cancellation for full-duplex communications with imperfect channel state information," IEEE Access, vol. 5, pp. 9277-9290, 2017.

[49] S. Khaledian, F. Farzami, B. Smida, and D. Erricolo, "Inherent self-interference 
cancellation for in-band full-duplex single-antenna systems," IEEE Transactions on Microwave Theory and Techniques, vol. 66, no. 6, pp. 2842-2850, June 2018.

[50] A. T. Le, L. C. Tran, and X. Huang, "Cyclostationary analysis of analog least mean square loop for self-interference cancellation in in-band full-duplex systems," IEEE Communications Letters, vol. 21, no. 12, pp. 2738-2741, Dec 2017.

[51] S. A. Ayati, D. Mandal, B. Bakkaloglu, and S. Kiaei, "Integrated quasicirculator with RF leakage cancellation for full-duplex wireless transceivers," IEEE Transactions on Microwave Theory and Techniques, vol. 66, no. 3, pp. 1421-1430, March 2018.

[52] S. J. Watt, E. A. Alwan, W. Khalil, and J. L. Volakis, "Wideband selfinterference cancellation filter for simultaneous transmit and receive systems," in 2015 IEEE International Symposium on Antennas and Propagation USNC/URSI National Radio Science Meeting, July 2015, pp. 129-130.

[53] D. Bharadia, E. McMilin, and S. Katti, "Full duplex radios," in ACM SIGCOMM Computer Communication Review, vol. 43, 2013, pp. 375-386.

[54] J. I. Choi, M. Jain, K. Srinivasan, P. Levis, and S. Katti, "Achieving single channel, full duplex wireless communication," in Proceedings of the Sixteenth Annual International Conference on Mobile Computing and Networking. ACM, 2010, pp. 1-12. 
[55] T. Riihonen and R. Wichman, "Analog and digital self-interference cancellation in full-duplex MIMO-OFDM transceivers with limited resolution in A/D conversion," in 2012 Conference Record of the Forty Sixth Asilomar Conference on Signals, Systems and Computers (ASILOMAR), Nov 2012, pp. 45-49.

[56] Y. Hua, P. Liang, Y. Ma, A. C. Cirik, and Q. Gao, "A method for broadband full-duplex MIMO radio," IEEE Signal Processing Letters, vol. 19, no. 12, pp. 793-796, Dec 2012.

[57] M. P. Fitz, T. R. Halford, I. Hossain, and S. W. Enserink, "Towards simultaneous radar and spectral sensing," in 2014 IEEE International Symposium on Dynamic Spectrum Access Networks (DYSPAN), April 2014, pp. 15-19.

[58] N. M. Gowda and A. Sabharwal, "JointNull: Combining partial analog cancellation with transmit beamforming for large-antenna full-duplex wireless systems," IEEE Transactions on Wireless Communications, vol. 17, no. 3, pp. 2094-2108, March 2018.

[59] S. Bojja-Venkatakrishnan, E. A. Alwan, and J. L. Volakis, "Wideband RF and analog self-interference cancellation filter for simultaneous transmit and receive system," in 2017 IEEE International Symposium on Antennas and Propagation USNC/URSI National Radio Science Meeting, July 2017, pp. 933-934.

[60] A. Kiayani, M. Z. Waheed, L. Anttila, M. Abdelaziz, D. Korpi, V. Syrjl, M. Kosunen, K. Stadius, J. Ryynnen, and M. Valkama, "Adaptive nonlinear RF 
cancellation for improved isolation in simultaneous transmit-receive systems," IEEE Transactions on Microwave Theory and Techniques, vol. 66, no. 5, pp. 2299-2312, May 2018.

[61] M. Heino, D. Korpi, T. Huusari, E. Antonio-Rodriguez, S. Venkatasubramanian, T. Riihonen, L. Anttila, C. Icheln, K. Haneda, R. Wichman, and M. Valkama, "Recent advances in antenna design and interference cancellation algorithms for in-band full duplex relays," IEEE Communications Magazine, vol. 53, no. 5, pp. 91-101, May 2015.

[62] M. S. Amjad, H. Nawaz, K. zsoy, . Grbz, and I. Tekin, "A low-complexity fullduplex radio implementation with a single antenna," IEEE Transactions on Vehicular Technology, vol. 67, no. 3, pp. 2206-2218, March 2018.

[63] H. Li, J. V. Kerrebrouck, O. Caytan, H. Rogier, J. Bauwelinck, P. Demeester, and G. Torfs, "Self-interference cancellation enabling high-throughput shortreach wireless full-duplex communication," IEEE Transactions on Wireless Communications, vol. 17, no. 10, pp. 6475-6486, Oct 2018.

[64] M. Chung, M. S. Sim, J. Kim, D. K. Kim, and C. Chae, "Prototyping real-time full duplex radios," IEEE Communications Magazine, vol. 53, no. 9, pp. 56-63, September 2015. 
[65] S. Li and R. D. Murch, "An investigation into baseband techniques for singlechannel full-duplex wireless communication systems," IEEE Transactions on Wireless Communications, vol. 13, no. 9, pp. 4794-4806, Sept 2014.

[66] D. Korpi, M. Heino, C. Icheln, K. Haneda, and M. Valkama, "Compact inband full-duplex relays with beyond $100 \mathrm{~dB}$ self-interference suppression: Enabling techniques and field measurements," IEEE Transactions on Antennas and Propagation, vol. 65, no. 2, pp. 960-965, Feb 2017.

[67] Y. Y. Kang, B. Kwak, and J. H. Cho, "An optimal full-duplex AF relay for joint analog and digital domain self-interference cancellation," IEEE Transactions on Communications, vol. 62, no. 8, pp. 2758-2772, Aug 2014.

[68] A. Masmoudi and T. Le-Ngoc, "Channel estimation and self-interference cancelation in full-duplex communication systems," IEEE Transactions on Vehicular Technology, vol. 66, no. 1, pp. 321-334, Jan 2017.

[69] S. B. Venkatakrishnan, E. A. Alwan, and J. L. Volakis, "Wideband RF selfinterference cancellation circuit for phased array simultaneous transmit and receive systems," IEEE Access, vol. 6, pp. 3425-3432, 2018.

[70] K. L. Scherer, S. J. Watt, E. A. Alwan, A. A. Akhiyat, B. Dupaix, W. Khalil, and J. L. Volakis, "Simultaneous transmit and receive system architecture with 
four stages of cancellation," in 2015 IEEE International Symposium on Antennas and Propagation USNC/URSI National Radio Science Meeting, July 2015, pp. $520-521$.

[71] D. Korpi, L. Anttila, V. Syrjl, and M. Valkama, "Widely linear digital selfinterference cancellation in direct-conversion full-duplex transceiver," IEEE Journal on Selected Areas in Communications, vol. 32, no. 9, pp. 1674-1687, Sep. 2014.

[72] F. J. Soriano-Irigaray, J. S. Fernandez-Prat, F. J. Lopez-Martinez, E. MartosNaya, O. Cobos-Morales, and J. T. Entrambasaguas, "Adaptive self-interference cancellation for full duplex radio: Analytical model and experimental validation," IEEE Access, vol. 6, pp. 65 018-65 026, 2018.

[73] Y. Liu, X. Quan, W. Pan, and Y. Tang, "Digitally assisted analog interference cancellation for in-band full-duplex radios," IEEE Communications Letters, vol. 21, no. 5, pp. 1079-1082, May 2017.

[74] Z. Li, Y. Xia, W. Pei, K. Wang, and D. P. Mandic, "An augmented nonlinear LMS for digital self-interference cancellation in full-duplex direct-conversion transceivers," IEEE Transactions on Signal Processing, vol. 66, no. 15, pp. 4065-4078, Aug 2018. 
[75] S. Huberman and T. Le-Ngoc, "Self-interference pricing-based MIMO fullduplex precoding," IEEE Wireless Communications Letters, vol. 3, no. 6, pp. 549-552, Dec 2014.

[76] E. Everett, C. Shepard, L. Zhong, and A. Sabharwal, "SoftNull: Many-antenna full-duplex wireless via digital beamforming," IEEE Transactions on Wireless Communications, vol. 15, no. 12, pp. 8077-8092, Dec 2016.

[77] S. E. Johnston and P. D. Fiore, "Full-duplex communication via adaptive nulling," in 2013 Asilomar Conference on Signals, Systems and Computers, Nov 2013, pp. 1628-1631.

[78] A. Masmoudi and T. Le-Ngoc, "A maximum-likelihood channel estimator for self-interference cancelation in full-duplex systems," IEEE Transactions on Vehicular Technology, vol. 65, no. 7, pp. 5122-5132, July 2016.

[79] E. Ahmed and A. M. Eltawil, "All-digital self-interference cancellation technique for full-duplex systems," IEEE Transactions on Wireless Communications, vol. 14, no. 7, pp. 3519-3532, July 2015.

[80] J. Li, H. Zhang, and M. Fan, "Digital self-interference cancellation based on independent component analysis for co-time co-frequency full-duplex communication systems," IEEE Access, vol. 5, pp. 10 222-10 231, 2017. 
[81] R. Li, A. Masmoudi, and T. Le-Ngoc, "Self-interference cancellation with nonlinearity and phase-noise suppression in full-duplex systems," IEEE Transactions on Vehicular Technology, vol. 67, no. 3, pp. 2118-2129, March 2018.

[82] K. Komatsu, Y. Miyaji, and H. Uehara, "Basis function selection of frequencydomain Hammerstein self-interference canceller for in-band full-duplex wireless communications," IEEE Transactions on Wireless Communications, vol. 17, no. 6, pp. 3768-3780, June 2018.

[83] D. Liang, P. Xiao, G. Chen, M. Ghoraishi, and R. Tafazolli, "Digital selfinterference cancellation for full-duplex MIMO systems," in 2015 International Wireless Communications and Mobile Computing Conference (IWCMC), Aug 2015, pp. 403-407.

[84] Y. Zhang, E. DallAnese, and G. B. Giannakis, "Distributed optimal beamformers for cognitive radios robust to channel uncertainties," IEEE Transactions on Signal Processing, vol. 60, no. 12, pp. 6495-6508, Dec 2012.

[85] Y. Shim, W. Choi, and H. Park, "Beamforming design for full-duplex two-way amplify-and-forward MIMO relay," IEEE Transactions on Wireless Communications, vol. 15, no. 10, pp. 6705-6715, Oct 2016.

[86] Z. Wen, S. Wang, X. Liu, and J. Zou, "Joint relay-user beamforming design in a full-duplex two-way relay channel," IEEE Transactions on Vehicular Technology, vol. 66, no. 3, pp. 2874-2879, March 2017. 
[87] N. Ayir and P. Ubaidulla, "Transceiver design for MIMO full-duplex two-way relay network," IEEE Wireless Communications Letters, vol. 7, no. 5, pp. 772775 , Oct 2018 .

[88] C. Lin and W. Wu, "Linear transceiver design for full-duplex MIMO relay systems: A non-iterative approach," IEEE Wireless Communications Letters, vol. 6, no. 4, pp. 518-521, Aug 2017.

[89] A. C. Cirik, L. Zhou, and T. Ratnarajah, "Linear transceiver design with perantenna power constraints in full-duplex multi-user MIMO systems," IEEE Wireless Communications Letters, vol. 5, no. 4, pp. 412-415, Aug 2016.

[90] B. Chun and H. Park, "A spatial-domain joint-nulling method of selfinterference in full-duplex relays," IEEE Communications Letters, vol. 16, no. 4, pp. 436-438, April 2012.

[91] T. M. Kim, H. J. Yang, and A. J. Paulraj, "Distributed sum-rate optimization for full-duplex MIMO system under limited dynamic range," IEEE Signal Processing Letters, vol. 20, no. 6, pp. 555-558, June 2013.

[92] G. Zheng, "Joint beamforming optimization and power control for full-duplex MIMO two-way relay channel," IEEE Transactions on Signal Processing, vol. 63, no. 3, pp. 555-566, Feb 2015.

[93] H. H. M. Tam, H. D. Tuan, and D. T. Ngo, "Successive convex quadratic programming for quality-of-service management in full-duplex MU-MIMO multicell 
networks," IEEE Transactions on Communications, vol. 64, no. 6, pp. 23402353, June 2016.

[94] B. Hu, C. Hua, C. Chen, X. Ma, and X. Guan, "MUBFP: Multiuser beamforming and partitioning for sum capacity maximization in MIMO systems," IEEE Transactions on Vehicular Technology, vol. 66, no. 1, pp. 233-245, Jan 2017.

[95] J. Kim, W. Choi, and H. Park, "Beamforming for full-duplex multiuser MIMO systems," IEEE Transactions on Vehicular Technology, vol. 66, no. 3, pp. 24232432, March 2017.

[96] J. Park, J. Hong, and S. Beak, "Optimal beamforming with limited feedback for millimeter-wave in-band full-duplex mobile x-haul network," IEEE Access, vol. 6, pp. $51038-51048,2018$.

[97] Y. Li, P. Fan, L. Liu, and Y. Yi, "Distributed MIMO precoding for in-band full-duplex wireless backhaul in heterogeneous networks," IEEE Transactions on Vehicular Technology, vol. 67, no. 3, pp. 2064-2076, March 2018.

[98] A. C. Cirik, R. Wang, Y. Hua, and M. Latva-aho, "Weighted sum-rate maximization for full-duplex MIMO interference channels," IEEE Transactions on Communications, vol. 63, no. 3, pp. 801-815, March 2015.

[99] A. C. Cirik, R. Wang, Y. Rong, and Y. Hua, "MSE-based transceiver designs for full-duplex MIMO cognitive radios," IEEE Transactions on Communications, vol. 63, no. 6, pp. 2056-2070, June 2015. 
[100] A. C. Cirik, S. Biswas, O. Taghizadeh, and T. Ratnarajah, "Robust transceiver design in full-duplex MIMO cognitive radios," IEEE Transactions on Vehicular Technology, vol. 67, no. 2, pp. 1313-1330, Feb 2018.

[101] A. C. Cirik, O. Taghizadeh, L. Lampe, R. Mathar, and Y. Hua, "Linear transceiver design for full-duplex multi-cell MIMO systems," IEEE Access, vol. 4, pp. 4678-4689, 2016.

[102] C. Lin, F. Tseng, W. Wu, and R. Y. Chang, "Nonlinear transceiver designs for full-duplex MIMO relay systems," IEEE Transactions on Communications, vol. 65, no. 11, pp. 4632-4645, Nov 2017.

[103] U. Ugurlu, T. Riihonen, and R. Wichman, "Optimized in-band full-duplex MIMO relay under single-stream transmission," IEEE Transactions on Vehicular Technology, vol. 65, no. 1, pp. 155-168, Jan 2016.

[104] Y. Jiang, F. C. M. Lau, I. W. Ho, H. Chen, and Y. Huang, "Maxmin weighted downlink SINR with uplink SINR constraints for full-duplex MIMO systems," IEEE Transactions on Signal Processing, vol. 65, no. 12, pp. 3277-3292, June 2017.

[105] T. M. Berhane, W. Meng, L. Chen, G. D. Jobir, and C. Li, "SLNR-based precoding for single cell full-duplex MU-MIMO systems," IEEE Transactions on Vehicular Technology, vol. 66, no. 9, pp. 7877-7887, Sep. 2017. 
[106] M. J. Rahman, A. C. Cirik, and L. Lampe, "Power-efficient transceiver design for full-duplex MIMO multi-cell systems with CSI uncertainty," IEEE Access, vol. 5, pp. $22689-22703,2017$.

[107] T. Chang, Y. Liu, and S. Lin, "QoS-based linear transceiver optimization for full-duplex multiuser communications," IEEE Transactions on Signal Processing, vol. 66, no. 9, pp. 2300-2313, May 2018.

[108] S. Han, Y. Zhang, W. Meng, and H. Chen, "Self-interference-cancelation-based SLNR precoding design for full-duplex relay-assisted system," IEEE Transactions on Vehicular Technology, vol. 67, no. 9, pp. 8249-8262, Sep. 2018.

[109] A. C. Cirik, M. J. Rahman, and L. Lampe, "Robust fairness transceiver design for a full-duplex MIMO multi-cell system," IEEE Transactions on Communications, vol. 66, no. 3, pp. 1027-1041, March 2018.

[110] S. W. Peters and R. W. Heath, "Cooperative algorithms for MIMO interference channels," IEEE Transactions on Vehicular Technology, vol. 60, no. 1, pp. 206218, Jan 2011.

[111] X. Li, Y. Sun, N. Zhao, F. R. Yu, and Z. Xu, "A novel interference alignment scheme with a full-duplex MIMO relay," IEEE Communications Letters, vol. 19, no. 10, pp. 1798-1801, Oct 2015.

[112] P. Aquilina and T. Ratnarajah, "Linear interference alignment in full-duplex 
MIMO networks with imperfect CSI," IEEE Transactions on Communications, vol. 65, no. 12, pp. 5226-5243, Dec 2017.

[113] M. A. Ahmed, C. C. Tsimenidis, and A. F. A. Rawi, "Performance analysis of full-duplex-MRC-MIMO with self-interference cancellation using null-spaceprojection," IEEE Transactions on Signal Processing, vol. 64, no. 12, pp. 30933105, June 2016.

[114] D. Hwang, Y. Choi, and T. Lee, "Optimization of zero forcing beamformer for the full duplex relay system," IEEE Communications Letters, vol. 20, no. 8, pp. 1583-1586, Aug 2016.

[115] A. Almradi and K. A. Hamdi, "MIMO full-duplex relaying in the presence of co-channel interference," IEEE Transactions on Vehicular Technology, vol. 66, no. 6, pp. 4874-4885, June 2017.

[116] A. Almradi, P. Xiao, and K. A. Hamdi, "Hop-by-hop ZF beamforming for MIMO full-duplex relaying with co-channel interference," IEEE Transactions on Communications, vol. 66, no. 12, pp. 6135-6149, Dec 2018.

[117] D. Hwang, J. Yang, and S. S. Nam, "SINR maximizing beamforming schemes for the full duplex amplify-and-forward relay channel," IEEE Access, vol. 5, pp. $18987-18998,2017$.

[118] Z. Xiao, P. Xia, and X. Xia, "Full-duplex millimeter-wave communication," IEEE Wireless Communications, vol. 24, no. 6, pp. 136-143, Dec 2017. 
[119] X. Xia, D. Zhang, K. Xu, W. Ma, and Y. Xu, "Hardware impairments aware transceiver for full-duplex massive MIMO relaying," IEEE Transactions on Signal Processing, vol. 63, no. 24, pp. 6565-6580, Dec 2015.

[120] H. Shen, C. Liu, W. Xu, and C. Zhao, "Optimized full-duplex MIMO DF relaying with limited dynamic range," IEEE Access, vol. 5, pp. 20726-20735, 2017.

[121] T. Riihonen, S. Werner, and R. Wichman, "Mitigation of loopback selfinterference in full-duplex MIMO relays," IEEE Transactions on Signal Processing, vol. 59, no. 12, pp. 5983-5993, Dec 2011.

[122] B. P. Day, A. R. Margetts, D. W. Bliss, and P. Schniter, "Full-duplex bidirectional MIMO: Achievable rates under limited dynamic range," IEEE Transactions on Signal Processing, vol. 60, no. 7, pp. 3702-3713, July 2012.

[123] E. Antonio-Rodrguez, R. López-Valcarce, T. Riihonen, S. Werner, and R. Wichman, "SINR optimization in wideband full-duplex MIMO relays under limited dynamic range," in 2014 IEEE 8th Sensor Array and Multichannel Signal Processing Workshop (SAM), June 2014, pp. 177-180.

[124] — - "Subspace-constrained SINR optimization in MIMO full-duplex relays under limited dynamic range," in 2015 IEEE 16th International Workshop on Signal Processing Advances in Wireless Communications (SPAWC), June 2015, pp. 281-285. 
[125] T. Guo and B. Wang, "Joint transceiver beamforming design for end-to-end optimization in full-duplex MIMO relay system with self-interference," IEEE Communications Letters, vol. 20, no. 9, pp. 1733-1736, Sept 2016.

[126] O. Taghizadeh, A. C. Cirik, and R. Mathar, "Hardware impairments aware transceiver design for full-duplex amplify-and-forward MIMO relaying," IEEE Transactions on Wireless Communications, vol. 17, no. 3, pp. 1644-1659, March 2018.

[127] I. T. Cummings, T. J. Schulz, J. P. Doane, and T. C. Havens, "An informationtheoretic approach to partitioning simultaneous transmit and receive digital phased arrays," in Proc. 2018 IEEE Radar Conference, Oklahoma City, OK, USA, 2018.

[128] — , "Optimizing the information-theoretic partitioning of simultaneous transmit and receive phased arrays," in Proc. 2018 IEEE International Symposium on Antennas and Propagation, Boston, MA, USA, 2018.

[129] I. T. Cummings, T. J. Schulz, J. P. Doane, S. A. R. Zekavat, and T. C. Havens, "Information-theoretic optimization of full-duplex communication between digital phased arrays," in Proc. 56th Annual Allerton Conference on Communication, Control, and Computing (Allerton), Monticello, IL, USA, Oct 2018.

[130] C. Fulton, M. Yeary, D. Thompson, J. Lake, and A. Mitchell, "Digital phased 
arrays: Challenges and opportunities," Proceedings of the IEEE, vol. 104, no. 3, pp. 487-503, March 2016.

[131] H. M. Wang, X. Q. Gao, B. Jiang, X. H. You, and W. Hong, "Efficient MIMO channel estimation using complementary sequences," IET Communications, vol. 1, no. 5, pp. 962-969, Oct 2007.

[132] N. Vasconcelos, "The Rayleigh quotient," http://www.svcl.ucsd.edu/courses/ ece271B-F09/handouts/Dimensionality3.pdf.

[133] G. F. Khodaei, J. Nourinia, and C. Ghobadi, "A practical miniaturized Uslot patch antenna with enhanced bandwidth," Progress In Electromagnetics Research B, vol. 3, pp. 47-62, 2008.

[134] A. Sabharwal, P. Schniter, D. Guo, D. W. Bliss, S. Rangarajan, and R. Wichman, "In-band full-duplex wireless: Challenges and opportunities," IEEE Journal on Selected Areas in Communications, vol. 32, no. 9, pp. 1637-1652, Sep. 2014.

[135] W. D. Wirth, "Long term coherent integration for a floodlight radar," in Proceedings International Radar Conference, May 1995, pp. 698-703.

[136] R. Schmidt, "Multiple emitter location and signal parameter estimation," IEEE Transactions on Antennas and Propagation, vol. 34, no. 3, pp. 276-280, March 1986. 
[137] R. Roy and T. Kailath, "ESPRIT-estimation of signal parameters via rotational invariance techniques," IEEE Transactions on Acoustics, Speech, and Signal Processing, vol. 37, no. 7, pp. 984-995, July 1989.

[138] R. Hamza and K. Buckley, "Resolution enhanced ESPRIT," IEEE Transactions on Signal Processing, vol. 42, no. 3, pp. 688-691, March 1994.

[139] M. I. Miller and D. R. Fuhrmann, "Maximum-likelihood narrow-band direction finding and the EM algorithm," IEEE Transactions on Acoustics, Speech, and Signal Processing, vol. 38, no. 9, pp. 1560-1577, Sep. 1990.

[140] P. Stoica and A. Nehorai, "Performance study of conditional and unconditional direction-of-arrival estimation," IEEE Transactions on Acoustics, Speech, and Signal Processing, vol. 38, no. 10, pp. 1783-1795, Oct 1990.

[141] S. T. Smith, "Statistical resolution limits and the complexified Cramér-Rao bound," IEEE Transactions on Signal Processing, vol. 53, no. 5, pp. 1597-1609, May 2005.

[142] J. Li and R. T. Compton, "Maximum likelihood angle estimation for signals with known waveforms," IEEE Transactions on Signal Processing, vol. 41, no. 9, pp. 2850-2862, Sep. 1993.

[143] M. Pesavento and A. B. Gershman, "Maximum-likelihood direction-of-arrival estimation in the presence of unknown nonuniform noise," IEEE Transactions on Signal Processing, vol. 49, no. 7, pp. 1310-1324, July 2001. 
[144] H. Nguyen and H. L. Van Trees, "Comparison of performance bounds for DOA estimation," in IEEE Seventh SP Workshop on Statistical Signal and Array Processing, June 1994, pp. 313-316.

[145] K. L. Bell, Y. Ephraim, and H. L. Van Trees, "Explicit Ziv-Zakai lower bound for bearing estimation," IEEE Transactions on Signal Processing, vol. 44, no. 11, pp. 2810-2824, Nov 1996.

[146] F. Athley, "Threshold region performance of maximum likelihood direction of arrival estimators," IEEE Transactions on Signal Processing, vol. 53, no. 4, pp. 1359-1373, April 2005.

[147] C. D. Richmond, "Mean-squared error and threshold SNR prediction of maximum-likelihood signal parameter estimation with estimated colored noise covariances," IEEE Transactions on Information Theory, vol. 52, no. 5, pp. 2146-2164, May 2006.

[148] J. M. Kantor, C. D. Richmond, D. W. Bliss, and B. Correll, "Mean-squarederror prediction for Bayesian direction-of-arrival estimation," IEEE Transactions on Signal Processing, vol. 61, no. 19, pp. 4729-4739, Oct 2013.

[149] F. Athley, "Optimization of element positions for direction finding with sparse arrays," in Proceedings of the 11th IEEE Signal Processing Workshop on Statistical Signal Processing (Cat. No.01TH8563), Aug 2001, pp. 516-519. 
[150] M. Gavish and A. J. Weiss, "Array geometry for ambiguity resolution in direction finding," IEEE Transactions on Antennas and Propagation, vol. 44, no. 6, pp. 889-895, June 1996.

[151] A. N. Mirkin and L. H. Sibul, "Cramér-Rao bounds on angle estimation with a two-dimensional array," IEEE Transactions on Signal Processing, vol. 39, no. 2, pp. 515-517, Feb 1991.

[152] H. Gazzah and S. Marcos, "Cramér-Rao bounds for antenna array design," IEEE Transactions on Signal Processing, vol. 54, no. 1, pp. 336-345, Jan 2006.

[153] A. Dogandzic and A. Nehorai, "Cramér-Rao bounds for estimating range, velocity, and direction with an active array," IEEE Transactions on Signal Processing, vol. 49, no. 6, pp. 1122-1137, June 2001.

[154] O. Lange and B. Yang, "Array geometry optimization for direction-of-arrival estimation including subarrays and tapering," in 2010 International ITG Workshop on Smart Antennas (WSA), Feb 2010, pp. 135-142.

[155] U. Oktel and R. L. Moses, "A Bayesian approach to array geometry design," IEEE Transactions on Signal Processing, vol. 53, no. 5, pp. 1919-1923, May 2005.

[156] S. K. Cheung, W. H. Weedon, and C. P. Caldwell, "High isolation Lange-ferrite circulators with NF suppression for simultaneous transmit and receive," in 2010 IEEE MTT-S International Microwave Symposium, May 2010, pp. 1352-1355. 
[157] S. K. Cheung, T. P. Halloran, W. H. Weedon, and C. P. Caldwell, "MMICbased quadrature hybrid quasi-circulators for simultaneous transmit and receive," IEEE Transactions on Microwave Theory and Techniques, vol. 58, no. 3, pp. 489-497, March 2010.

[158] T. Dinc and H. Krishnaswamy, "A 28GHz magnetic-free non-reciprocal passive CMOS circulator based on spatio-temporal conductance modulation," in 2017 IEEE International Solid-State Circuits Conference (ISSCC), Feb 2017, pp. 294-295.

[159] H. Lin, E. Long, J. Sosnowski, and V. Jamnejad, "Design of a new smaller lighter Faraday rotator for ACERAD radar QOTL," in 2015 IEEE Aerospace Conference, March 2015, pp. 1-7.

[160] A. Melzer, F. Starzer, H. Jger, and M. Huemer, "Real-time mitigation of shortrange leakage in automotive FMCW radar transceivers," IEEE Transactions on Circuits and Systems II: Express Briefs, vol. 64, no. 7, pp. 847-851, July 2017.

[161] A. Melzer, A. Onic, and M. Huemer, "Self-adaptive short-range leakage canceler for automotive FMCW radar transceivers," in 2018 15th European Radar Conference (EuRAD), Sep. 2018, pp. 26-29.

[162] T. Venkatamuni, L. S. S. Sarma, and A. T. Kalghatgi, "Adaptive reflected power canceller for single antenna FMCW radar," in 2009 Asia Pacific Microwave Conference, Dec 2009, pp. 1841-1844. 
[163] B. Ku, O. Inac, M. Chang, H. Yang, and G. M. Rebeiz, "A high-linearity 76-85$\mathrm{GHz}$ 16-element 8-transmit/8-receive phased-array chip with high isolation and flip-chip packaging," IEEE Transactions on Microwave Theory and Techniques, vol. 62, no. 10, pp. 2337-2356, Oct 2014.

[164] H. L. V. Trees and K. L. Bell, Bayesian Bounds for Parameter Estimation and Nonlinear Filtering/Tracking. Wiley, 2007.

[165] R. L. Haupt and S. E. Haupt, Practical Genetic Algorithms. Wiley-Interscience, 1998.

[166] R. L. Haupt and D. H. Werner, Genetic Algorithms in Electromagnetics. WileyInterscience, 2007.

[167] S. Haykin, Adaptive Filter Theory, 5th ed. Pearson, 2014.

[168] B. Widrow and J. McCool, "A comparison of adaptive algorithms based on the methods of steepest descent and random search," IEEE Transactions on Antennas and Propagation, vol. 24, no. 5, pp. 615-637, Sep. 1976.

[169] I. S. Reed, J. D. Mallett, and L. E. Brennan, "Rapid convergence rate in adaptive arrays," IEEE Transactions on Aerospace and Electronic Systems, vol. AES-10, no. 6, pp. 853-863, Nov 1974.

[170] N. Li, W. Zhu, and H. Han, "Digital interference cancellation in single channel, full duplex wireless communication," in 2012 8th International Conference on 
Wireless Communications, Networking and Mobile Computing, Sep. 2012, pp. $1-4$.

[171] D. Korpi, Y. Choi, T. Huusari, L. Anttila, S. Talwar, and M. Valkama, "Adaptive nonlinear digital self-interference cancellation for mobile inband full-duplex radio: Algorithms and RF measurements," in 2015 IEEE Global Communications Conference (GLOBECOM), Dec 2015, pp. 1-7.

[172] M. AghababaeeTafreshi, M. Koskela, D. Korpi, P. Jskelinen, M. Valkama, and J. Takala, "Software defined radio implementation of adaptive nonlinear digital self-interference cancellation for mobile inband full-duplex radio," in 2016 IEEE Global Conference on Signal and Information Processing (GlobalSIP), Dec 2016, pp. 733-737.

[173] P. Ferrand and M. Duarte, "Multi-tap digital canceller for full-duplex applications," in 2017 IEEE 18th International Workshop on Signal Processing Advances in Wireless Communications (SPAWC), July 2017, pp. 1-5.

[174] C. An and H. Ryu, "Design and performance evaluation of SSD (simultaneous single band duplex) system using RF cancellation and digital cancellation," in 2014 International Conference on Information and Communication Technology Convergence (ICTC), Oct 2014, pp. 960-963.

[175] J. Tamminen, M. Turunen, D. Korpi, T. Huusari, Y. Choi, S. Talwar, and M. Valkama, "Digitally-controlled RF self-interference canceller for full-duplex 
radios," in 2016 24th European Signal Processing Conference (EUSIPCO), Aug 2016, pp. $783-787$.

[176] T. Huusari, Y. Choi, P. Liikkanen, D. Korpi, S. Talwar, and M. Valkama, "Wideband self-adaptive RF cancellation circuit for full-duplex radio: Operating principle and measurements," in 2015 IEEE 81st Vehicular Technology Conference (VTC Spring), May 2015, pp. 1-7.

[177] Seunghyeon Kim, Youngil Jeon, G. Noh, Youn-Ok Park, Ilgyu Kim, and Hyunchol Shin, "A 2.59-GHz RF self-interference cancellation circuit with wide dynamic range for in-band full-duplex radio," in 2016 IEEE MTT-S International Microwave Symposium (IMS), May 2016, pp. 1-4.

[178] M. Adams and V. K. Bhargava, "Use of the recursive least squares filter for self interference channel estimation," in 2016 IEEE 84th Vehicular Technology Conference (VTC-Fall), Sep. 2016, pp. 1-4.

[179] A. Gebhard, O. Lang, M. Lunglmayr, C. Motz, R. S. Kanumalli, C. Auer, T. Paireder, M. Wagner, H. Pretl, and M. Huemer, "A robust nonlinear RLS type adaptive filter for second-order-intermodulation distortion cancellation in FDD LTE and 5G direct conversion transceivers," IEEE Transactions on Microwave Theory and Techniques, vol. 67, no. 5, pp. 1946-1961, May 2019.

[180] M. Emara, K. Roth, L. Gomes Baltar, M. Faerber, and J. Nossek, "Nonlinear digital self-interference cancellation with reduced complexity for full duplex 
systems," in WSA 2017; 21th International ITG Workshop on Smart Antennas, March 2017, pp. 1-6.

[181] E. Ahmed and A. M. Eltawil, "All-digital self-interference cancellation technique for full-duplex systems," IEEE Transactions on Wireless Communications, vol. 14, no. 7, pp. 3519-3532, July 2015.

[182] H. Vogt, G. Enzner, and A. Sezgin, "State-space adaptive nonlinear selfinterference cancellation for full-duplex communication," IEEE Transactions on Signal Processing, vol. 67, no. 11, pp. 2810-2825, June 2019.

[183] G. Enzner, "Bayesian inference model for applications of time-varying acoustic system identification," in 2010 18th European Signal Processing Conference, Aug 2010, pp. 2126-2130.

[184] A. Balatsoukas-Stimming, "Non-linear digital self-interference cancellation for in-band full-duplex radios using neural networks," in 2018 IEEE 19th International Workshop on Signal Processing Advances in Wireless Communications (SPAWC), June 2018, pp. 1-5.

[185] A. Masmoudi and T. Le-Ngoc, "A maximum-likelihood channel estimator for self-interference cancelation in full-duplex systems," IEEE Transactions on Vehicular Technology, vol. 65, no. 7, pp. 5122-5132, July 2016.

[186] L. Anttila, D. Korpi, E. Antonio-Rodríguez, R. Wichman, and M. Valkama, 
"Modeling and efficient cancellation of nonlinear self-interference in MIMO fullduplex transceivers," in 2014 IEEE Globecom Workshops (GC Wkshps), Dec 2014, pp. $777-783$.

[187] D. Korpi, L. Anttila, and M. Valkama, "Nonlinear self-interference cancellation in MIMO full-duplex transceivers under crosstalk," EURASIP Journal on Wireless Communications and Networking, vol. 2017, no. 1, p. 24, Feb 2017. [Online]. Available: https://doi.org/10.1186/s13638-017-0808-4

[188] E. Antonio-Rodríguez, R. López-Valcarce, T. Riihonen, S. Werner, and R. Wichman, "Adaptive self-interference cancellation in wideband full-duplex decode-and-forward MIMO relays," in 2013 IEEE 14th Workshop on Signal Processing Advances in Wireless Communications (SPAWC), June 2013, pp. $370-374$.

[189] J. S. Lemos, F. A. Monteiro, I. Sousa, and A. Rodrigues, "Full-duplex relaying in MIMO-OFDM frequency-selective channels with optimal adaptive filtering," in 2015 IEEE Global Conference on Signal and Information Processing (GlobalSIP), Dec 2015, pp. 1081-1085.

[190] I. Cummings, T. Schulz, T. Havens, and J. Doane, "Neural networks for realtime adaptive beamforming in simultaneous transmit and receive digital phased arrays," in Proc. 2019 IEEE Int. Symp. on Phased Array Syst. and Technol. (PAST), Waltham, MA, USA, Oct 2019. 
[191] E. W. Weisstein, "Wolfram mathworld: Complex multiplication," http:// mathworld.wolfram.com/ComplexMultiplication.html.

[192] — - "Wolfram mathworld: Complex division," http://mathworld.wolfram. com/ComplexDivision.html.

[193] — , "Wolfram mathworld: Square root," http://mathworld.wolfram.com/ SquareRoot.html.

[194] The Mathworks, Inc., "MATLAB R2017b help: mldivide, \," 2017.

[195] A. V. Frolov, "Cholesky decomposition: Serial complexity of the algorithm," https://algowiki-project.org/en/Cholesky_decomposition\#Serial_ complexity_of_the_algorithm, Feb 2016.

[196] —- "Forward substitution: Serial complexity of the algorithm," https://algowiki-project.org/en/Forward_substitution\#Serial_complexity_ of_the_algorithm, Feb 2016.

[197] — , "Backward substitution: Serial complexity of the algorithm," https://algowiki-project.org/en/Backward_substitution\#Serial_complexity_of_the_algorithm, Feb 2016.

[198] F. Mezzadri, "How to generate random matrices from the classical compact groups," 2006. 
[199] M. van Leeuwen (https://math.stackexchange.com/users/18880/marc-van leeuwen), "Basis to hyperplane," Mathematics Stack Exchange, uRL:https://math.stackexchange.com/q/1909570 (version: 2017-04-13). [Online]. Available: https://math.stackexchange.com/q/1909570

[200] A. P. (https://math.stackexchange.com/users/90996/algebraic pavel), "Algorithm to find an orthogonal basis (orthogonal to a given vector)," Mathematics Stack Exchange, uRL:https://math.stackexchange.com/q/712030 (version: 2014-03-14). [Online]. Available: https://math.stackexchange.com/q/712030

[201] M. Parker, Understanding Peak Floating-Point Performance Claims, Intel Corporation, 2017.

[202] TOP500.org, "Top 10 sites for November 2019," https://www.top500.org/lists/ 2019/11/, Nov 2019.

[203] S. Haykin, Adaptive Filter Theory, ser. Prentice-Hall Information and System Sciences Series. Prentice-Hall, 2002.

[204] R. A. Monzingo, R. L. Haupt, and T. W. Miller, Introduction to Adaptive Arrays, 2nd ed. Scitech Publishing, 2011.

[205] G. Casella and R. L. Berger, Statistical Inference, 2nd ed. The Wadsworth Group, 2002. 
[206] davidleejy, "Answer to: Intuitive explanation of a definition of the fisher information," https://math.stackexchange.com/questions/265917/ intuitive-explanation-of-a-definition-of-the-fisher-information, Jul 2016.

[207] H. Cramér, Mathematical Methods of Statistics. Princeton University Press, 1946. 


\section{Appendix A}

\section{Beamforming}

Generally speaking, beamforming is the process of using the known spatial relationships between measured data points to filter them in a way that amplifies the response of the system in a particular direction or directions of interest, and nulls the response in other directions.

The simplest form of beamforming uses knowledge of the relative positioning of the antennas in an array to calculate the required phase shifts that will create a plane wave propagating in the desired direction with respect to the antenna array. The process of calculating these phase shifts is best illustrated with an example. Assume that there are $N$ antennas spaced $\Delta m$ apart, as shown in Fig. A.1. This arrangement of antennas is referred to as a uniform linear array (ULA). In order to generate a narrowband 


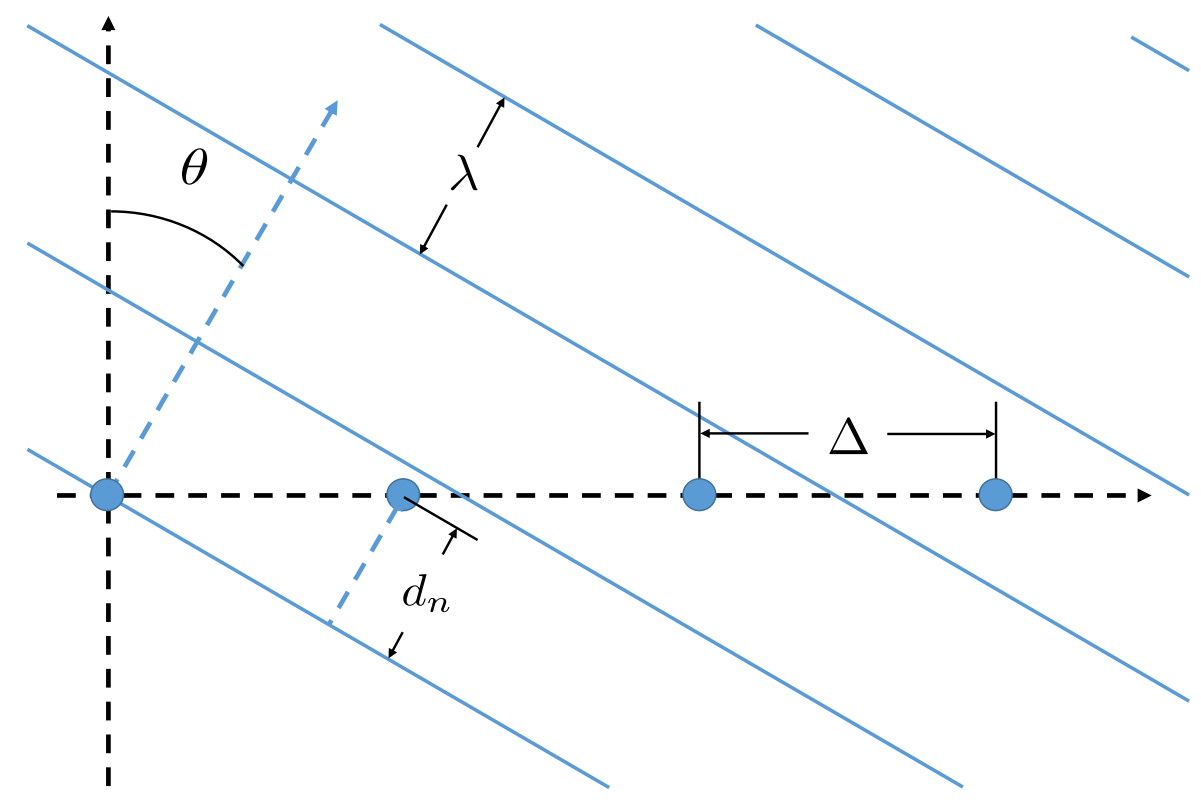

Figure A.1: Geometry of the basic beamforming problem for a uniform linear array.

plane wave propagating at azimuth angle $\theta$ from array broadside, all that is required is to shift the phase of the signal at each antenna so that the wavefronts will align along a plane perpendicular to the desired direction of propagation. The perpendicular distance to that planar wavefront (i.e. the distance parallel to the blue-dashed arrow) from antenna $n$ is

$$
d_{n}=-\Delta(n-1) \sin \theta
$$

where we have assumed that the phase at the first antenna is 0 (i.e. $\phi_{1}=0$ ) and the negative sign indicates that the distance to the wavefront is opposite the direction of propagation. Therefore, the equivalent phase shift for the $n$th antenna is

$$
\phi_{n}=-\frac{2 \pi}{\lambda} \Delta(n-1) \sin \theta
$$


The vector of complex weights that can be applied to the complex baseband signal at each antenna is

$$
\mathbf{q}=\exp \left(-j \frac{2 \pi}{\lambda} \Delta[01 \cdots N-1] \sin \theta\right)
$$

and is often referred to as a beamforming vector or beamformer. The symbol $\mathbf{q}$ is used here because this beamformer is the default beamformer in a quiescent (or interference-free) environment. These phase shifts will create a plane wave in the direction of interest when used to shift transmitted signals, and they will also properly align signals received at the same antennas to create maximum gain in the direction of interest $\theta$.

Up to this point, the discussion has focused on determining the beamformer that will produce the highest transmit or receive gain in the direction of interest. It is also possible for beamformers to reduce the effects of interference (i.e. unwanted signals) and noise while still achieving high gain in the direction of interest. It is most natural to discuss these concepts in terms of a receive beamformer, but the same formulation and concepts apply directly to the equivalent expressions for a transmit beamformer. The performance of a beamformer that is trying to focus on a desired signal and reject interference and noise is measured by the signal-to-interference-plus-noise-ratio (SINR), given as

$$
\mathrm{SINR}=\frac{P_{s}}{P_{i+n}}
$$


$P_{s}$ is the power of the signal component in the receive beamformed signal and $P_{i+n}$ is the power of the interference and noise components in the receive beamformed signal. For a given beamformer $\mathbf{b}$, the SINR can be written

$$
\operatorname{SINR}=\frac{\mathbf{b}^{\mathrm{H}} \mathbf{q q}{ }^{\mathrm{H}} \mathbf{b}}{\mathbf{b}^{\mathrm{H}} \mathbf{R}_{i+n} \mathbf{b}},
$$

where $\mathbf{R}_{i+n}$ is the correlation matrix of the interference and noise signals at the antennas. Given this formulation, the problem of choosing a beamformer $\mathbf{b}$ that maximizes SINR becomes an optimization problem. In this case, the expression for SINR is a Generalized Rayleigh Quotient in terms of the beamformer $\mathbf{b}$, which can be converted to a Rayleigh Quotient. First, the eigendecomposition of $\mathbf{R}_{i+n}$ must be calculated,

$$
\mathbf{R}_{i+n}=\mathbf{U} \boldsymbol{\Lambda} \mathbf{U}^{\mathrm{H}} .
$$

Because $\mathbf{R}_{i+n}$ is a correlation matrix, it is necessarily positive semidefinite and all the entries of the diagonal eigenvalue matrix $\boldsymbol{\Lambda}$ are non-negative, so it can be factored into its square root by simply taking the square root of the diagonal entries,

$$
\mathbf{R}_{i+n}=\mathbf{U} \boldsymbol{\Lambda}^{1 / 2} \boldsymbol{\Lambda}^{1 / 2} \mathbf{U}^{\mathrm{H}} .
$$

Plugging this representation of $\mathbf{R}_{i+n}$ into our expression for SINR, we obtain

$$
\operatorname{SINR}=\frac{\mathbf{b}^{\mathrm{H}} \mathbf{q q} \mathbf{q}^{\mathrm{H}} \mathbf{b}}{\mathbf{b}^{\mathrm{H}} \mathbf{U} \boldsymbol{\Lambda}^{1 / 2} \boldsymbol{\Lambda}^{1 / 2} \mathbf{U}^{\mathrm{H}} \mathbf{b}} .
$$


Note that if we choose

$$
\mathbf{a}=\boldsymbol{\Lambda}^{1 / 2} \mathbf{U}^{\mathrm{H}} \mathbf{b},
$$

we can rewrite $(\mathrm{A} .8)$ as

$$
\begin{aligned}
\operatorname{SINR} & =\frac{\mathbf{a}^{\mathrm{H}} \boldsymbol{\Lambda}^{-1 / 2} \mathbf{U}^{\mathrm{H}} \mathbf{q} \mathbf{q}^{\mathrm{H}} \mathbf{U} \boldsymbol{\Lambda}^{-1 / 2} \mathbf{a}}{\mathbf{a}^{\mathrm{H}} \mathbf{a}} \\
& =\frac{\mathbf{a}^{\mathrm{H}} \mathbf{c} \mathbf{c}^{\mathrm{H}} \mathbf{a}}{\mathbf{a}^{\mathrm{H}} \mathbf{a}} \text {, s.t. } \mathbf{c}=\boldsymbol{\Lambda}^{-1 / 2} \mathbf{U}^{\mathrm{H}} \mathbf{q}
\end{aligned}
$$

which is the Rayleigh Quotient we sought. By inspecting (A.10), we can see that this expression is maximized when we pick

$$
\hat{\mathbf{a}}=\alpha \mathbf{c}=\alpha \boldsymbol{\Lambda}^{-1 / 2} \mathbf{U}^{\mathrm{H}} \mathbf{q}
$$

where $\alpha$ is an arbitrary scale factor. This becomes clear when we recognize that A.10) is the magnitude squared of the dot product of $\mathbf{a}$ with the vector $\mathbf{c}$ normalized by the squared magnitude of $\mathbf{a}$. The dot product of $\mathbf{a}$ and $\mathbf{c}$ is maximized when $\mathbf{a}$ is parallel to $\mathbf{c}$, and our choice of magnitude for $\mathbf{a}$ is arbitrary. Then, solving (A.9) for $\mathbf{b}$ and substituting in $\hat{\mathbf{a}}$, we get

$$
\begin{aligned}
\hat{\mathbf{b}} & =\mathbf{U} \boldsymbol{\Lambda}^{-1 / 2} \hat{\mathbf{a}} \\
& =\alpha \mathbf{U} \boldsymbol{\Lambda}^{-1 / 2} \boldsymbol{\Lambda}^{-1 / 2} \mathbf{U}^{\mathrm{H}} \mathbf{q} \\
& =\alpha \mathbf{R}_{i+n}^{-1} \mathbf{q},
\end{aligned}
$$


which is the expression for the beamformer that maximizes the SINR of the received signal. The technique of calculating a beamformer that optimizes some chosen performance metric is often referred to as adaptive beamforming. This particular adaptive beamforming technique is often referred to as the Wiener optimal beamformer. It is widely used, and several more detailed descriptions and motivations for it are available in [203, 204]. 


\section{Appendix B}

\section{The Cramér-Rao Lower Bound and}

\section{The Bayesian Cramér-Rao Lower}

\section{Bound}

In many signal processing problems, we are faced with estimating some parameter $\theta$ given some vector of data samples $\mathbf{x}$. The Cramér-Rao Lower Bound (CRLB) and the Bayesian Cramér-Rao Lower Bound (BCRB) give us insight into the fundamental limits on how well we can estimate $\theta$ given our models for the data and the parameter. Within the context of the estimation problem, there are two broad categories of problems, based on whether the parameter $\theta$ is deterministic (but unknown) or random. In the first case (deterministic $\theta$ ), the only probabilistic information we have 
that relates the variables is the likelihood of the data given the parameter, $p(\mathbf{x} \mid \theta)$. Consider the structure of Bayes' Theorem,

$$
p(\theta \mid \mathbf{x})=\frac{p(\mathbf{x} \mid \theta) p(\theta)}{p(\mathbf{x})} .
$$

Note that the posterior distribution $p(\theta \mid \mathbf{x})$ fully describes the probability of any value of the parameter $\theta$ being the true value given some specific data set $\mathbf{x}$. If we had access to this distribution, we could simply plug in $\mathbf{x}$ and find the value of $\theta$ that maximized $p(\theta \mid \mathbf{x})$. However, in typical scenarios, there is no good way to model $p(\mathbf{x})$. In the first case (deterministic $\theta$ ), we do not have a definition for $p(\theta)$, and the CRLB sets the lower limit for the estimation accuracy of any signal processing technique. In the second case (random $\theta$ ), we have a meaningful prior $p(\theta)$ which can be used to inform our estimate of $\theta$ from $\mathbf{x}$, and the BCRB sets the lower limit for the estimation accuracy of any signal processing technique. Note that the presence of a prior brings our probabilistic model closer to the ideal posterior distribution. 


\section{B.1 Intuitive Derivation of the CRLB}

The basic approach to bounding the estimation error for both cases relies on the Cauchy-Schwarz Inquality, which for two random variables can be stated simply as

$$
\operatorname{Var}[X] \cdot \operatorname{Var}[Y] \geq(\operatorname{Cov}[X, Y])^{2} .
$$

For the first (nonrandom parameter) case, let $X=\hat{\theta}(\mathbf{x})-\theta$ be the error of the parameter estimate, which is a function of the data random data $\mathbf{x}$. We assume that the expected value of $\hat{\theta}(\mathbf{x})$ is $\theta$, which implies that the estimator is unbiased and that $\operatorname{Var}[\mathrm{X}]$ is the mean squared error (MSE) of the estimator. Let $Y=g(\mathbf{x} \mid \theta)$ be some arbitrary function of the data and parameter, which is necessarily a random variable because it is a function of at least one random variable. Note that in the case of nonrandom $\theta, g(\mathbf{x} \mid \theta)$ still contains $\theta$ as a variable, but it is not random. With these choices for $X$ and $Y$, let us rearrange the inequality into a slightly more useful form for our purposes,

$$
\operatorname{Var}[X] \geq \frac{(\operatorname{Cov}[X, Y])^{2}}{\operatorname{Var}[Y]}
$$

In words, this equation states that we can calculate a lower bound on the variance of a random variable by the following procedure: calculate the covariance of the first random variable with a second random variable (which may be easier to calculate), 
square the result, and then "divide out" or normalize by the variance of the second random variable. Specifically for our problem, we can lower bound the estimation error of an estimator by calculating the covariance-squared of the estimator with some function of the data, normalized by the variance of that function. What's left is to choose a function $g(\mathbf{x} \mid \theta)$. In order to determine a satisfactory choice for $g(\mathbf{x} \mid \theta)$, we start by considering the following expression,

$$
\frac{\partial}{\partial \theta} E_{\mathbf{x} \mid \theta}[\hat{\theta}(\mathbf{x})-\theta]=0
$$

which describes how the average estimation error changes with respect to the value of the actual parameter. If we bring the derivative inside the integral (expectation), we obtain

$$
\int_{\mathbf{x}} \hat{\theta}(\mathbf{x}) \frac{\partial}{\partial \theta} p(\mathbf{x} \mid \theta) d \mathbf{x}-\int_{\mathbf{x}} p(\mathbf{x} \mid \theta) d \mathbf{x}=0
$$

Note that the second term goes to 1 , which we can move to the right-hand side,

$$
\int_{\mathbf{x}} \hat{\theta}(\mathbf{x}) \frac{\partial}{\partial \theta} p(\mathbf{x} \mid \theta) d \mathbf{x}=1
$$

Multiplying and dividing by $p(\mathbf{x} \mid \theta)$ inside the integral (so that we can turn the expression back into an expectation), we obtain

$$
\int_{\mathbf{x}} \hat{\theta}(\mathbf{x}) \frac{1}{p(\mathbf{x} \mid \theta)} \frac{\partial}{\partial \theta} p(\mathbf{x} \mid \theta) p(\mathbf{x} \mid \theta) d \mathbf{x}=1
$$


which is equivalent to

$$
E_{\mathbf{x} \mid \theta}\left[\hat{\theta}(\mathbf{x}) \frac{\partial}{\partial \theta} \ln p(\mathbf{x} \mid \theta)\right]=1
$$

when we realize that $\frac{1}{p(\mathbf{x} \mid \theta)} \frac{\partial}{\partial \theta} p(\mathbf{x} \mid \theta)=\frac{\partial}{\partial \theta} \ln p(\mathbf{x} \mid \theta)$. Taking another look at $(\mathrm{B} .2)$, we realize that it is the covariance between the estimator and the function $\frac{\partial}{\partial \theta} \ln p(\mathbf{x} \mid \theta)$, noting that $E_{\mathbf{x} \mid \theta}\left[\frac{\partial}{\partial \theta} \ln p(\mathbf{x} \mid \theta)\right]=0$. For this reason, we choose $g(\mathbf{x} \mid \theta)=\frac{\partial}{\partial \theta} \ln p(\mathbf{x} \mid \theta)$. Plugging these results into our rearranged Cauchy-Schwarz Inequality, we obtain the Cramér-Rao Lower Bound (CRLB) for the MSE of an estimator for a nonrandom parameter,

$$
C R L B=\operatorname{Var}_{\mathbf{x} \mid \theta}[\hat{\theta}(\mathbf{x})-\theta] \geq \frac{1}{\operatorname{Var}_{\mathbf{x} \mid \theta}\left[\frac{\partial \ln p(\mathbf{x} \mid \theta)}{\partial \theta}\right]}
$$

This more intuitive derivation of the CRLB follows the derivations presented in [205] (found via [206]) and [207].

\section{B.1.1 CRLB Example}

As a brief example, consider the problem of estimating the mean $\mu$ of a Gaussian random variable $x$ from $K$ independent and identically distributed (iid) samples. The likelihood for the vector of samples given the mean is

$$
p(\mathbf{x} \mid \mu)=\frac{1}{\left(2 \pi \sigma^{2}\right)^{N / 2}} \exp \left(-\frac{\sum_{k=0}^{K-1}\left(x_{k}-\mu\right)^{2}}{2 \sigma^{2}}\right) .
$$


The partial of the log-likelihood is given by

$$
\frac{\partial \ln p(\mathbf{x} \mid \mu)}{\partial \mu}=\frac{1}{\sigma^{2}} \sum_{k=0}^{K-1} x_{k}-\mu
$$

Evaluating the variance of the partial of the log-likelihood (note that it has zero mean), we have

$$
\begin{aligned}
\operatorname{Var}_{\mathbf{x} \mid \mu}\left[\frac{\partial \ln p(\mathbf{x} \mid \mu)}{\partial \mu}\right] & =E_{\mathbf{x} \mid \mu}\left[\frac{1}{\sigma^{4}} \sum_{k=0}^{K-1} \sum_{l=0}^{K-1}\left(x_{k}-\mu\right)\left(x_{l}-\mu\right)\right] \\
& =\frac{K}{\sigma^{2}} .
\end{aligned}
$$

Inverting the result, we obtain the CRLB on the estimation error for the nonrandom mean of $K$ samples of a Gaussian random variable with variance $\sigma^{2}$

$$
C R L B=\frac{\sigma^{2}}{K}
$$

This equation is intuitively satisfying, as it states that the accuracy to which we can estimate the mean is proportional to the power of the noise in the measurements and inversely proportional to the number of samples we take. 


\section{B.2 Intuitive Derivation of the BCRB}

Now let's consider the second case, where the parameter $\theta$ is random and we have a distribution $p(\theta)$ that describes the randomness of the parameter. The question becomes, how does this extra information affect how well we can estimate the true value of the mean? To explore that question, we must extend our definition for the CRLB to account for the presence of the prior $p(\theta)$. Because the prior encodes information about the most probable value for the parameter, including the prior inherently biases our estimator $\hat{\theta}(\mathbf{x})$. Recall that the joint probability density function is equal to the product of the likelihood and the prior, $p(\mathbf{x}, \theta)=p(\mathbf{x} \mid \theta) p(\theta)$. It turns out that the intuition used in the derivation of the CRLB also applies to the random parameter case, and we can simply replace $p(\mathbf{x} \mid \theta)$ in $(\mathrm{B} .2)$ with $p(\mathbf{x}, \theta)$, yielding

$$
E_{\mathbf{x}, \theta}\left[\hat{\theta}(\mathbf{x}) \frac{\partial}{\partial \theta} \ln p(\mathbf{x}, \theta)\right]=1
$$

This leads us to choose $g(\mathbf{x}, \theta)=\frac{\partial}{\partial \theta} \ln p(\mathbf{x}, \theta)$, which leads to a similar final form for the bound. We refer to this bound as the Bayesian Cramér-Rao Bound (BCRB) because it accounts for the prior, and it is given by

$$
B C R B=\operatorname{Var}_{\mathbf{x}, \theta}[\hat{\theta}(\mathbf{x})-\theta] \geq \frac{1}{\operatorname{Var}_{\mathbf{x}, \theta}\left[\frac{\partial \ln p(\mathbf{x}, \theta)}{\partial \theta}\right]}
$$




\section{B.2.1 BCRB Example}

We now extend the mean estimation example above by including a prior on the mean,

$$
p(\mu)=\frac{1}{\sqrt{2 \pi \sigma_{m}^{2}}} \exp \left(-\frac{(\mu-m)^{2}}{2 \sigma_{m}^{2}}\right) .
$$

The derivative of the log-joint probability density function is given by

$$
\frac{\partial \ln p(\mathbf{x}, \mu)}{\partial \mu}=-\frac{1}{\sigma^{2}} \sum_{k=0}^{K-1}\left(x_{k}-\mu\right)-\frac{1}{\sigma_{m}^{2}}(\mu-m),
$$

and the variance of the derivative of the log-joint probability density function is

$$
\operatorname{Var}_{\mathbf{x}, \theta}\left[\frac{\partial \ln p(\mathbf{x}, \theta)}{\partial \theta}\right]=\frac{K}{\sigma^{2}}+\frac{1}{\sigma_{m}^{2}}
$$

Therefore, the BCRB for the given example problem is

$$
B C R B=\frac{1}{\frac{K}{\sigma^{2}}+\frac{1}{\sigma_{m}^{2}}} .
$$

Intuitively this is a satisfying result, because as the measurement noise approaches $\infty$, the information in the prior dominates the lower bound on the uncertainty in the estimate of $\mu$. Furthermore, as the measurement noise $\sigma^{2}$ decreases or the number of samples $K$ increases, the measurement noise dominates the lower bound. In other 
words, this result is equivalent to the previous example in the high signal-to-noise ratio (SNR) region, but approaches the variance in the prior as SNR decreases. This makes sense, because in the low SNR region the measurements contain little information about the location of $\mu$, and therefore any estimator must begin to rely more strongly on the information provided by the prior $p(\mu)$. 



\section{Appendix C}

\section{Genetic Algorithms}

Genetic Algorithms (GAs) are a class of optimization technique from the field of computational intelligence. There are many scenarios where traditional gradientbased optimization techniques might fail, especially with highly non-convex objective functions or integer input spaces. In order to overcome this limitation, the GA was designed to mimic a simplified version of the theoretical process of evolution or adaptation. The space of potential solutions to the optimization problem must first be encoded into a binary string. Then an objective function that can convert that input into a single score or performance metric must be developed. In some cases, the input encoding and/or the objective function arise naturally out of the problem. For example, the optimization problem may be over a binary- or integer-vector input space. The choice of input encoding must be done carefully to avoid obscuring the 


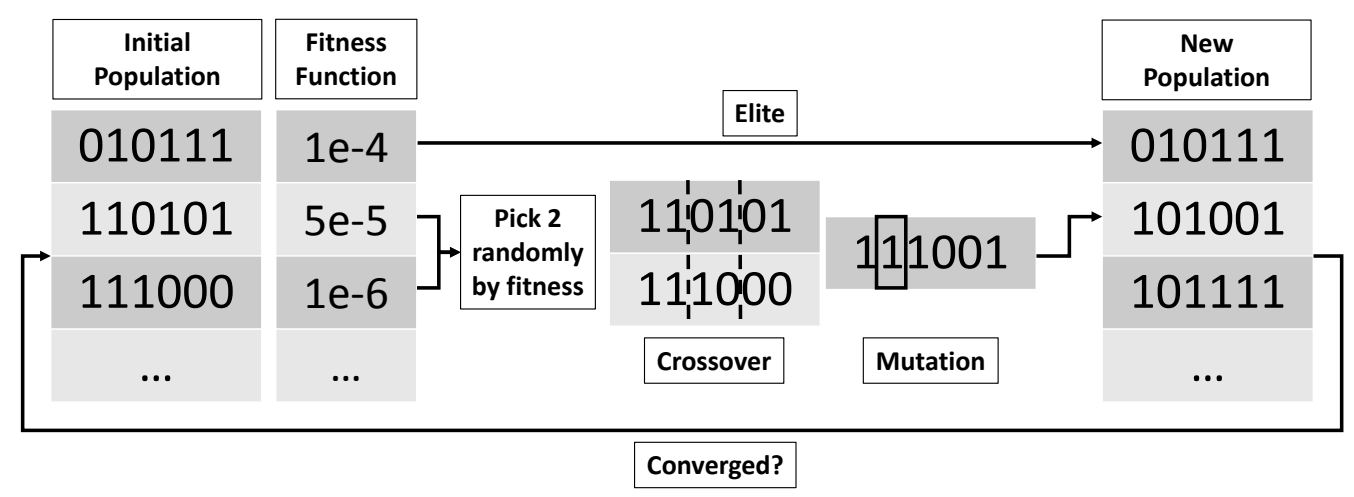

Figure C.1: Illustration of the key elements of the GA. Figure taken from Chapter 3 , based on a figure found in [2].

optimal solution. For example, if a continuous input is not discretized at a sufficient resolution to capture the nature of the solution space accurately, the GA may fail to find a near-optimal solution. Additionally, using Gray code ordering to encode discretizations of continuous inputs can make 1-bit genetic mutations more meaningful and local in the input space.

Fig. C.1 illustrates how the GA operates. To begin, a population of randomly generated individuals are created as potential solutions and their fitness is evaluated via the input encoding and fitness function created previously. Then pairs of individuals are selected to reproduce randomly with probability that is a function of their fitness. The reproduction process consists of a crossover and a mutation stage. During crossover, two random points in the binary string are selected, and the elements between or outside the points are exchanged with the reproduction partner to form a new individual. Then, the new individual is subjected to potential mutation at 
random, typically at a small number of locations and with low probability (e.g. 1-bit is mutated if any). The crossover operation allows successful input features (referred to as schema) from the high performing offspring of the previous population to be recombined into related individuals in the new population. This behavior in the GA is referred to as exploitation because the GA exploits the successes of previous individuals. The mutation operation allows the GA to explore the input space around successful individuals for as yet unseen high-performing schema, and thus is referred to as exploration. The relative strengths of exploration and exploitation in the GA can be tuned by changing the relative crossover and mutation probabilities. It is often considered prudent to carry over the best solution (or several top solutions) directly to the next generation without crossover or mutation. These individuals are referred to as elites, and keep the GA from losing sight of good solutions. Several books [2, 165, 166] provide a much more detailed explanation of the GA and the many design choices available to the user. 



\section{Appendix D}

\section{Letters of Permission}

The IEEE does not require individuals working on a thesis to obtain a formal reuse license, however, you may print out this statement to be used as a permission grant:

Requirements to be followed when using any portion (e.g., figure, graph, table, or textual material) of an IEEE copyrighted paper in a thesis:

1) In the case of textual material (e.g., using short quotes or referring to the work within these papers) users must give full credit to the original source (author, paper, publication) followed by the IEEE copyright line 2011 IEEE.

2) In the case of illustrations or tabular material, we require that the copyright line [Year of original publication] IEEE appear prominently with each reprinted figure 
and/or table.

3) If a substantial portion of the original paper is to be used, and if you are not the senior author, also obtain the senior author's approval.

Requirements to be followed when using an entire IEEE copyrighted paper in a thesis:

1) The following IEEE copyright/credit notice should be placed prominently in the references: [year of original publication] IEEE. Reprinted, with permission, from [author names, paper title, IEEE publication title, and month/year of publication]

2) Only the accepted version of an IEEE copyrighted paper can be used when posting the paper or your thesis on-line.

3) In placing the thesis on the author's university website, please display the following message in a prominent place on the website: In reference to IEEE copyrighted material which is used with permission in this thesis, the IEEE does not endorse any of Michigan Technological University's products or services. Internal or personal use of this material is permitted. If interested in reprinting/republishing IEEE copyrighted material for advertising or promotional purposes or for creating new collective works for resale or redistribution, please go to http://www.ieee.org/ publications_standards/publications/rights/rights_link.html to learn how to obtain a License from RightsLink. 
If applicable, University Microfilms and/or ProQuest Library, or the Archives of Canada may supply single copies of the dissertation. 